\title{
RECORDS OF WELLS AND CHEMICAL ANALYSES OF GROUND WATER IN CHARLES MIX AND DOUGLAS COUNTIES, SOUTH DAKOTA
}

By Kathleen M. Neitzert

U.S. GEOLOGICAL SURVEY

Open-File Report 95-153

Prepared in cooperation with the SOUTH DAKOTA DEPARTMENT OF ENVIRONMENT AND NATURAL RESOURCES

Rapid City, South Dakota 1995

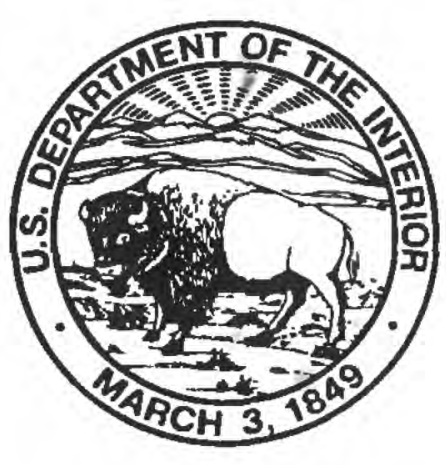




\section{U.S. DEPARTMENT OF THE INTERIOR}

BRUCE BABBITT, Secretary

U.S. GEOLOGICAL SURVEY

Gordon P. Eaton, Director

For additional information write to:

Copies of this report can be purchased from:

District Chief

U.S. Geological Survey

1608 Mt. View Rd.

Rapid City, SD 57702
U.S. Geological Survey

Earth Science Information Center

Open-File Reports Section

Box 25286, MS 517

Denver Federal Center

Denver, CO 80225 


\section{CONTENTS}

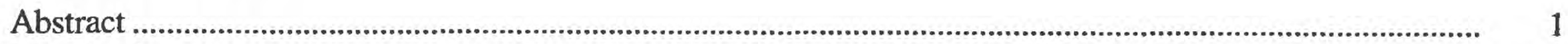

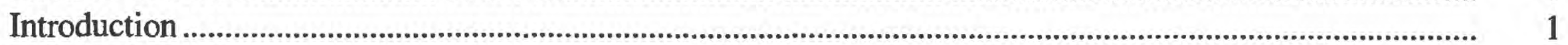

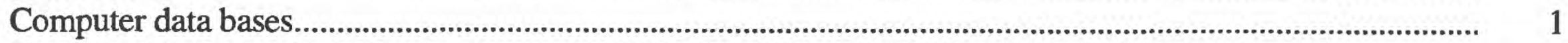

Data compilation and verification ............................................................................................................ 2

Location-numbering system.................................................................................................................. 2

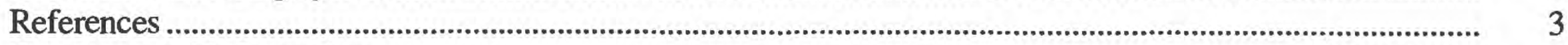

Supplemental information ............................................................................................................... 5

\section{ILLUSTRATIONS}

Figure 1. Map of South Dakota showing location of Charles Mix and Douglas Counties ................................. 2

2. Well-numbering diagram .................................................................................................................... 3

\section{TABLES}

Table 1. Records of wells and test holes in Charles Mix County ...................................................................... 7

2. Records of wells and test holes in Douglas County.......................................................................... 71

3. Chemical analyses of ground water in Charles Mix and Douglas Counties........................................ 98

\section{CONVERSION FACTORS AND VERTICAL DATUM}

\begin{tabular}{rcl}
\hline Multiply & By & To obtain \\
\hline foot (ft) & 0.3048 & meter \\
inch (in.) & 25.4 & millimeter \\
\hline
\end{tabular}

Temperature can be converted to degree Fahrenheit $\left({ }^{\circ} \mathrm{F}\right)$ or degree Celsius $\left({ }^{\circ} \mathrm{C}\right)$ by the following equations:

$$
\begin{gathered}
{ }^{\circ} \mathrm{F}=9 / 5\left({ }^{\circ} \mathrm{C}\right)+32 \\
{ }^{\circ} \mathrm{C}=5 / 9\left({ }^{\circ} \mathrm{F}-32\right)
\end{gathered}
$$

Sea level: In this report, "sea level" refers to the National Geodetic Vertical Datum of 1929--a geodetic datum derived from a general adjustment of the first-order level nets of the United States and Canada, formerly called Sea Level Datum of 1929. 


\title{
RECORDS OF WELLS AND CHEMICAL ANALYSES \\ OF GROUND WATER IN CHARLES MIX AND DOUGLAS COUNTIES, SOUTH DAKOTA
}

\author{
By Kathleen M. Neitzert
}

\section{ABSTRACT}

The U.S. Geological Survey, in cooperation with the South Dakota Department of Environment and Natural Resources, maintains a data base of information on water resources in the State of South Dakota. The ground-water site data for Charles Mix and Douglas Counties are presented in two tables, with the data organized by county and location. The chemical data for ground-water sites also are located in a table, organized by geologic unit and location.

\section{INTRODUCTION}

A multiple-year investigation was concluded in 1975 to determine the geology and water resources of Charles Mix and Douglas Counties in eastern South Dakota (fig. 1). The investigation, accomplished at the request of Charles Mix and Douglas Counties and the Fort Randall Conservancy Sub-District, was a cooperative effort by the South Dakota Geological Survey (SDGS) and the United States Geological Survey (USGS). A series of four reports were published by the SDGS and the USGS to summarize the findings of the investigation.

The previously published reports include: Sand and gravel resources in Charles Mix and Douglas Counties, South Dakota (Hedges, 1972); Major aquifers in Charles Mix and Douglas Counties, South Dakota (Kume, 1972); Geology and water resources of Charles Mix and Douglas Counties, South Dakota, Part I: Geology (Hedges, 1975), and Part II: Water resources (Kume, 1977).

Since the original investigation was completed, the USGS has cooperated with SDGS in a process of reviewing and verifying the original well, test-hole, and water-quality data. Furthermore, additional data have been collected. The purpose of this report is to compile existing well, test-hole, and chemical data for Charles Mix and Douglas Counties in one document.

\section{COMPUTER DATA BASES}

Physical, hydrologic, and geologic data for wells and test holes have been entered into computer storage in the Ground-Water Site-Inventory (GWSI) File of the U.S. Geological Survey's National Water Data Storage and Retrieval System (WATSTORE). The GWSI File is the national repository for data from sites where ground water has been, is, or can be withdrawn.

Chemical data for ground water have been entered into computer storage in the Water-Quality File of WATSTORE. Water-quality data consisting of chemical, physical, biological, and radiochemical parameters are stored in the Water-Quality File.

The Water Resources Division of the USGS maintains these WATSTORE data bases. In addition to its data processing, storage, and retrieval capabilities, WATSTORE has the capability of providing 


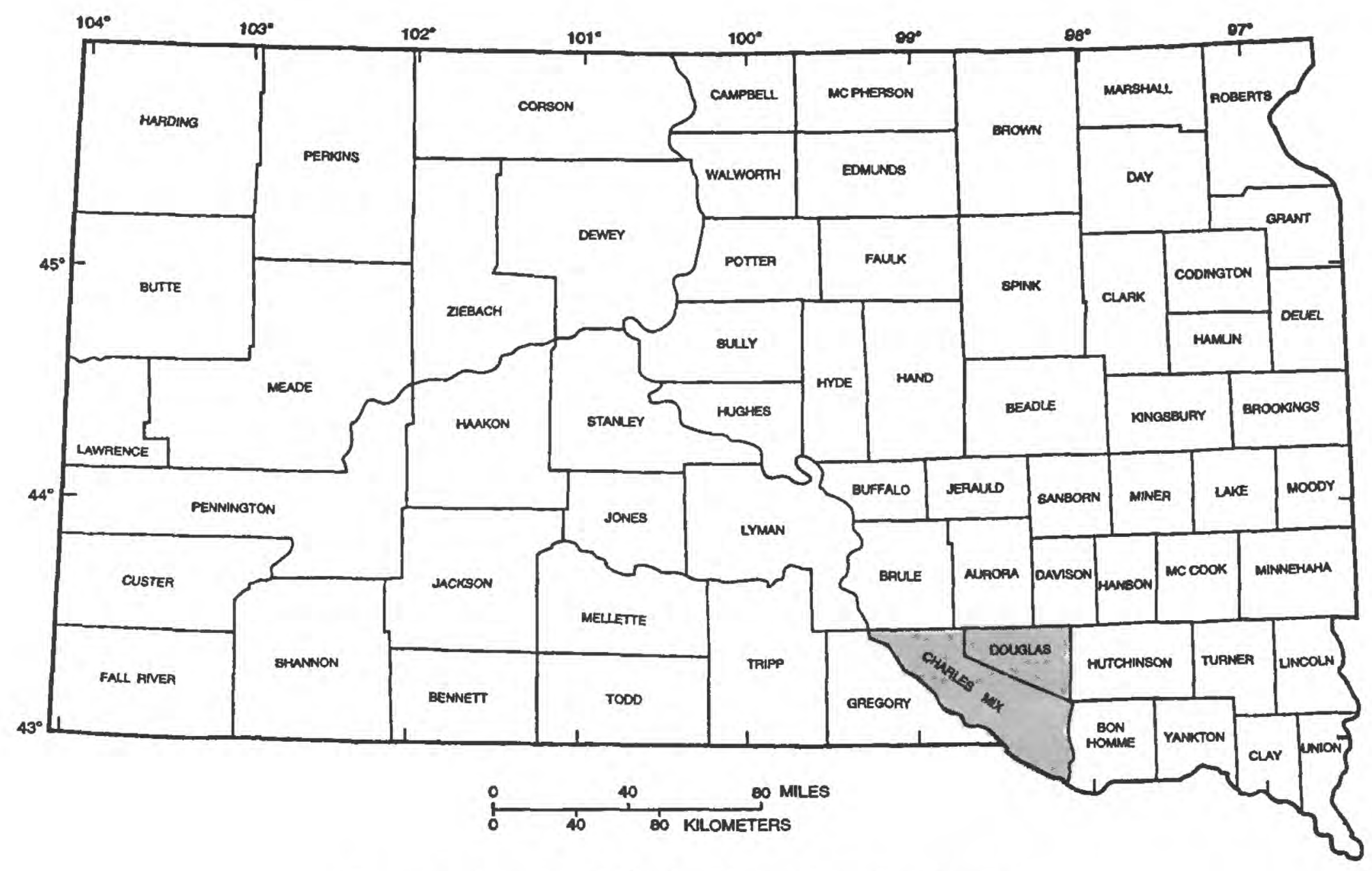

Figure 1. Location of Charles Mix and Douglas Counties.

computer-printed tables, computer-printed graphs, statistical analyses of data, and digital plots. The tables in this report were computer-generated and printed, using the WATSTORE system.

\section{DATA COMPILATION AND VERIFICATION}

All well and test-hole data collected for the original investigation of the geology and water resources of Charles Mix and Douglas Counties were verified by comparing the information in the computer data base with the information on the original well schedules. All well and test-hole data collected since 1977 were reviewed by a process of verifying the data against drillers' logs, interviewing landowners, and cross checking with data existing in the computer data base.

All chemical data for ground water were reviewed for accuracy and consistency using quality-control guidelines of the South Dakota District of the U.S. Geological Survey (U.S. Geological Survey, written commun., 1991).
Well and test-hole data for Charles Mix County are presented in table 1 in the Supplemental Information section at the end of this report. Well and test-hole data for Douglas County are presented in table 2 in the Supplemental Information section. The aquifer codes for each well or test hole were determined using the information published by Hedges (1975). The chemical data, sorted by aquifer, are presented in table 3 in the Supplemental Information section.

\section{LOCATION-NUMBERING SYSTEM}

Site locations are numbered according to the Federal land-survey system of eastern South Dakota (fig. 2). The local number consists of township followed by " $\mathrm{N}$," range followed by "W," and section number, followed by a maximum of four uppercase letters that indicate respectively, the 160-, 40-, 10-, and $21 / 2$-acre tract in which the well is located. These letters are assigned in a counterclockwise direction beginning with " $\mathrm{A}$ " in the northeast quarter. A serial number following the last letter is used to 
distinguish between wells in the same tract. Thus, well 100N67W14BAAA (fig. 2) is in the NE1/4, NE1/4, NE1/4, NW1/4, sec. 14, T. 100 N., R. 67 W.

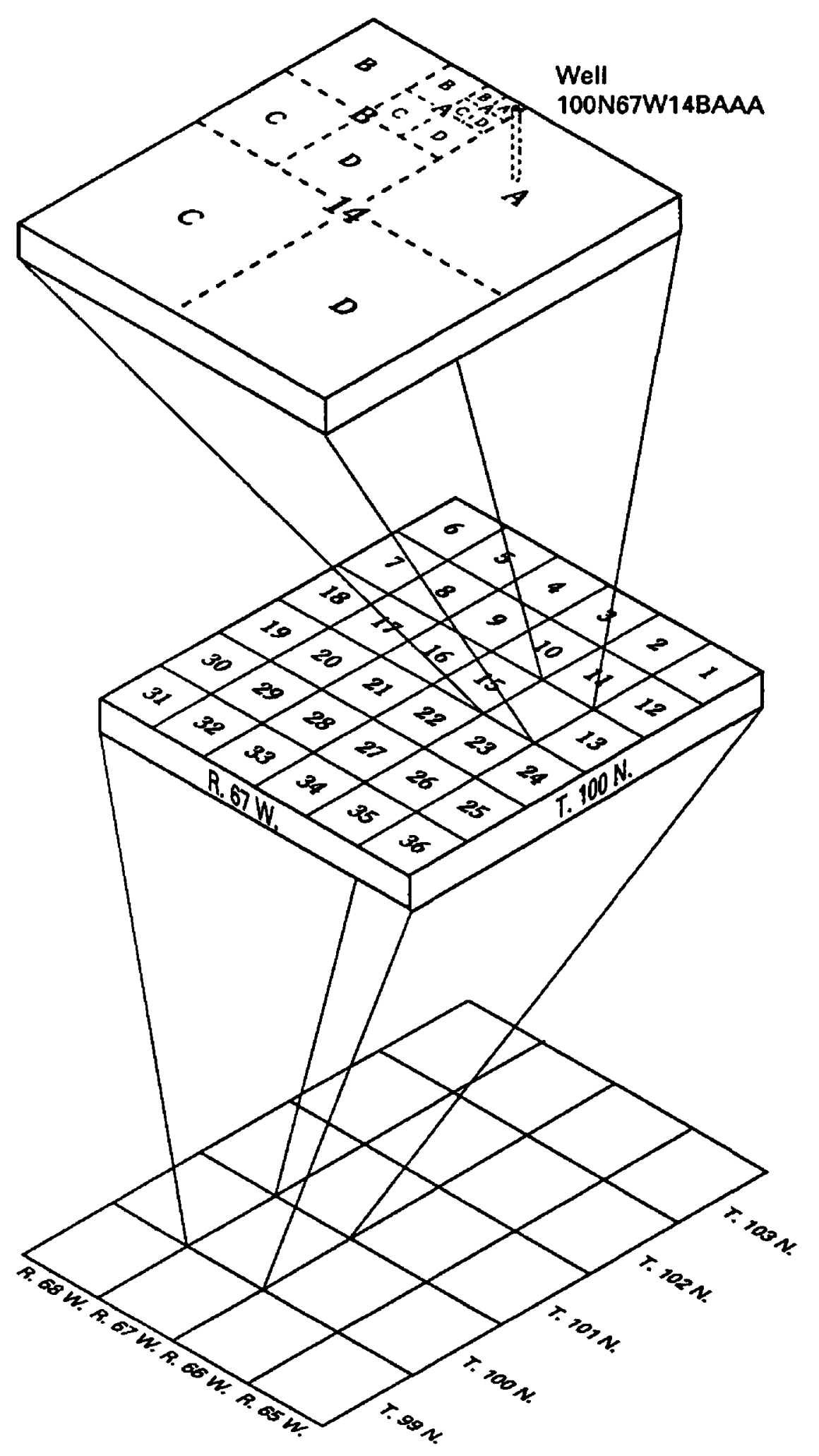

Figure 2. Well-numbering diagram.

\section{REFERENCES}

Hedges, L.S., 1972, Sand and gravel resources in Charles Mix and Douglas Counties, South Dakota: South Dakota Geological Survey Circular 42, 6 p.

----1975, Geology and water resources of Charles Mix and Douglas Counties, South Dakota, Part 1: Geology: South Dakota Geological Survey Bulletin $22,43 \mathrm{p}$.

Kume, Jack, 1972, Major aquifers in Charles Mix and Douglas Counties, South Dakota: South Dakota Geological Survey Information Pamphlet 2, 6 p.

----1977, Geology and water resources of Charles Mix and Douglas Counties, South Dakota, Part II: Water resources: South Dakota Geological Survey Bulletin 22, 31 p. 


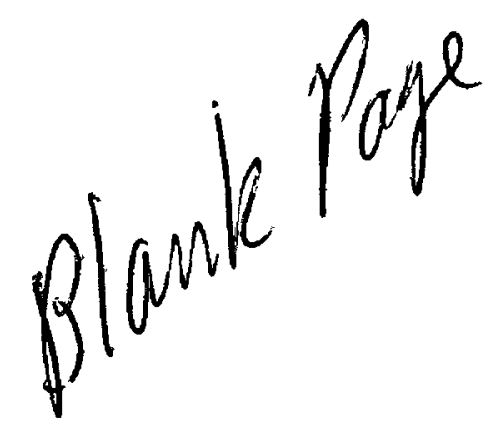

(4) 
SUPPLEMENTAL INFORMATION 


\section{Explanation}

The following abbreviations and codes are used in tables 1 and 2:

,-- no data

\section{Owner}

$\begin{array}{ll}\text { USGS } & \text { - U.S. Geological Survey } \\ \text { SDWR } & \text { - South Dakota Department of Environment and Natural Resources, } \\ & \text { Division of Water Rights } \\ \text { SDGS } & \text { - South Dakota Department of Environment and Natural Resources, } \\ & \text { Division of Geological Survey } \\ \text { BR } & \text { - Bureau of Reclamation } \\ \text { USCE } & \text { - U.S. Army Corps of Engineers } \\ \text { SDGFP } & - \text { South Dakota Department of Game, Fish and Parks } \\ \text { USFWS } & - \text { U.S. Fish \& Wildlife Service } \\ \text { USAF } & \text { - U.S. Air Force } \\ \text { BIA } & - \text { Bureau of Indian Affairs }\end{array}$

Principal Aquifer

$\begin{array}{ll}\text { 112CHTU } & \text { Choteau } \\ \text { 112CRSC } & \text { Corsica } \\ \text { 112DELM } & \text { Delmont } \\ \text { 112GEDS } & \text { Geddes } \\ \text { 112GENO } & \text { Greenwood } \\ \text { 112IRMD } & \text { Intermediate } \\ \text { 112PLSC } & \text { Pleistocene } \\ \text { 112TILL } & \text { Till } \\ \text { 211CDLL } & \text { Codell } \\ \text { 211DKOT } & \text { Dakota } \\ \text { 211NBRR } & \text { Niobrara } \\ \text { 211PIRR } & \text { Pierre Shale } \\ \text { 400SOUX } & \text { Sioux Quartzite }\end{array}$

Use of Water

$\mathrm{H}$ - domestic

S - stock

D - dewater

I - irrigation

Z - other

$\mathrm{U}$ - unused

$\mathrm{T}$ - institution

$\mathrm{P}$ - public supply

F - fire

$\mathrm{R}$ - recreation

$\mathrm{N}$ - industrial

E - power

Water level (feet)

Water level, in feet below or above ( + ) land surface

F - flowing

P - pumping

$\mathrm{R}$ - recently pumped 


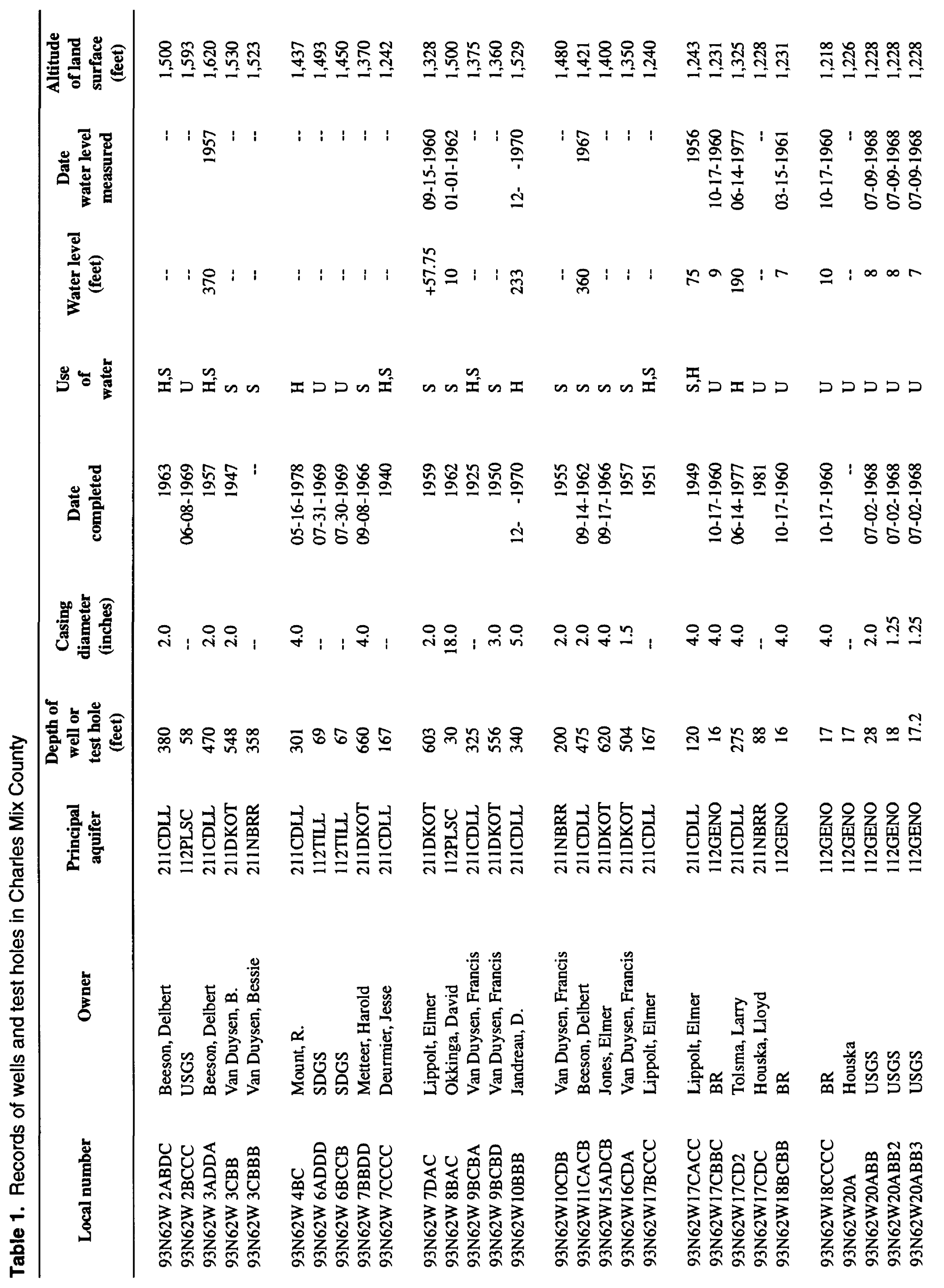

Table 1. Records of wells and test holes in Charles Mix County 7 


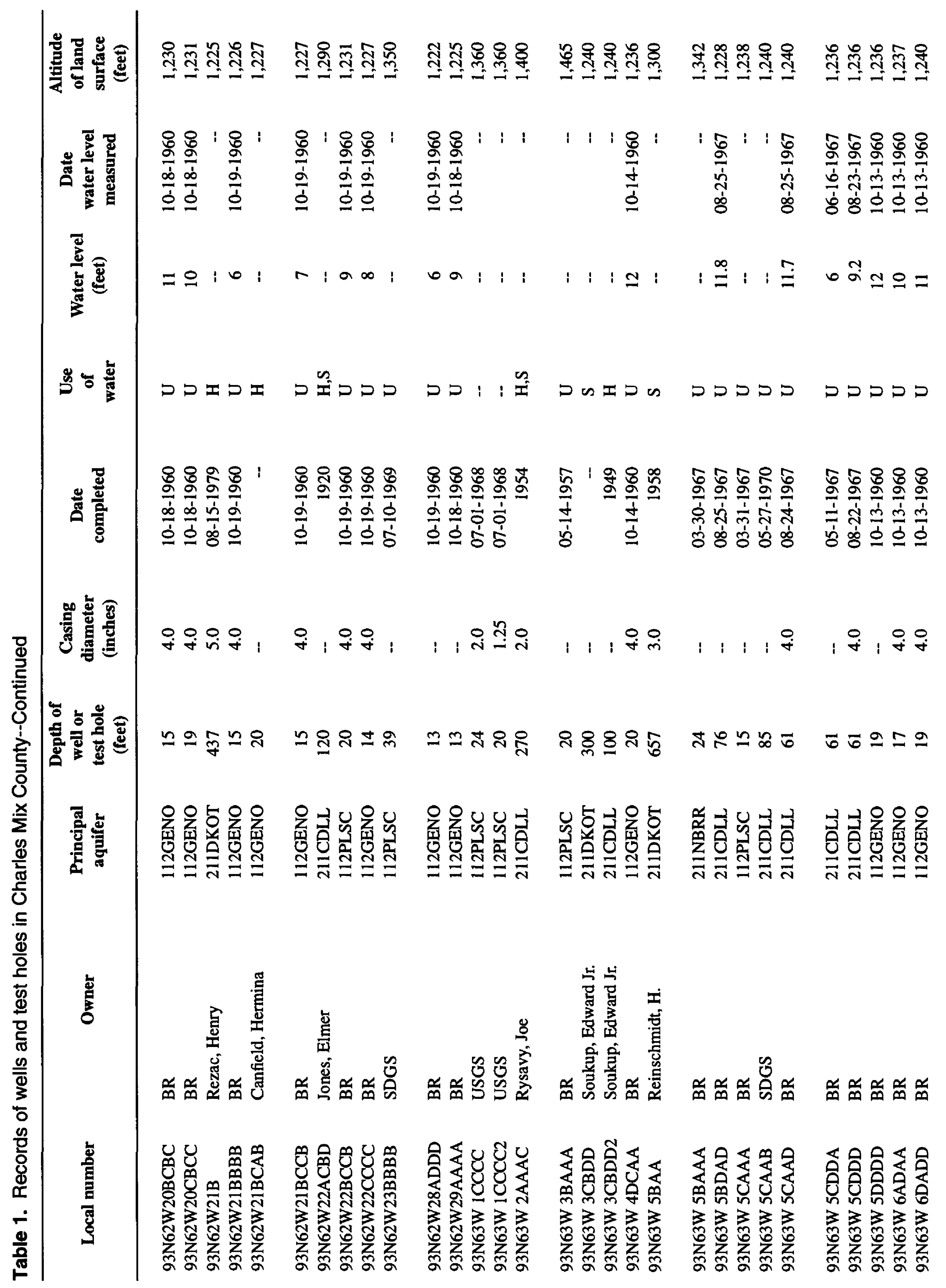

8 Records of Wells and Chemical Anslyses of Ground Water in Chsrles Mix snd Douglas Countiss, South Dakota 


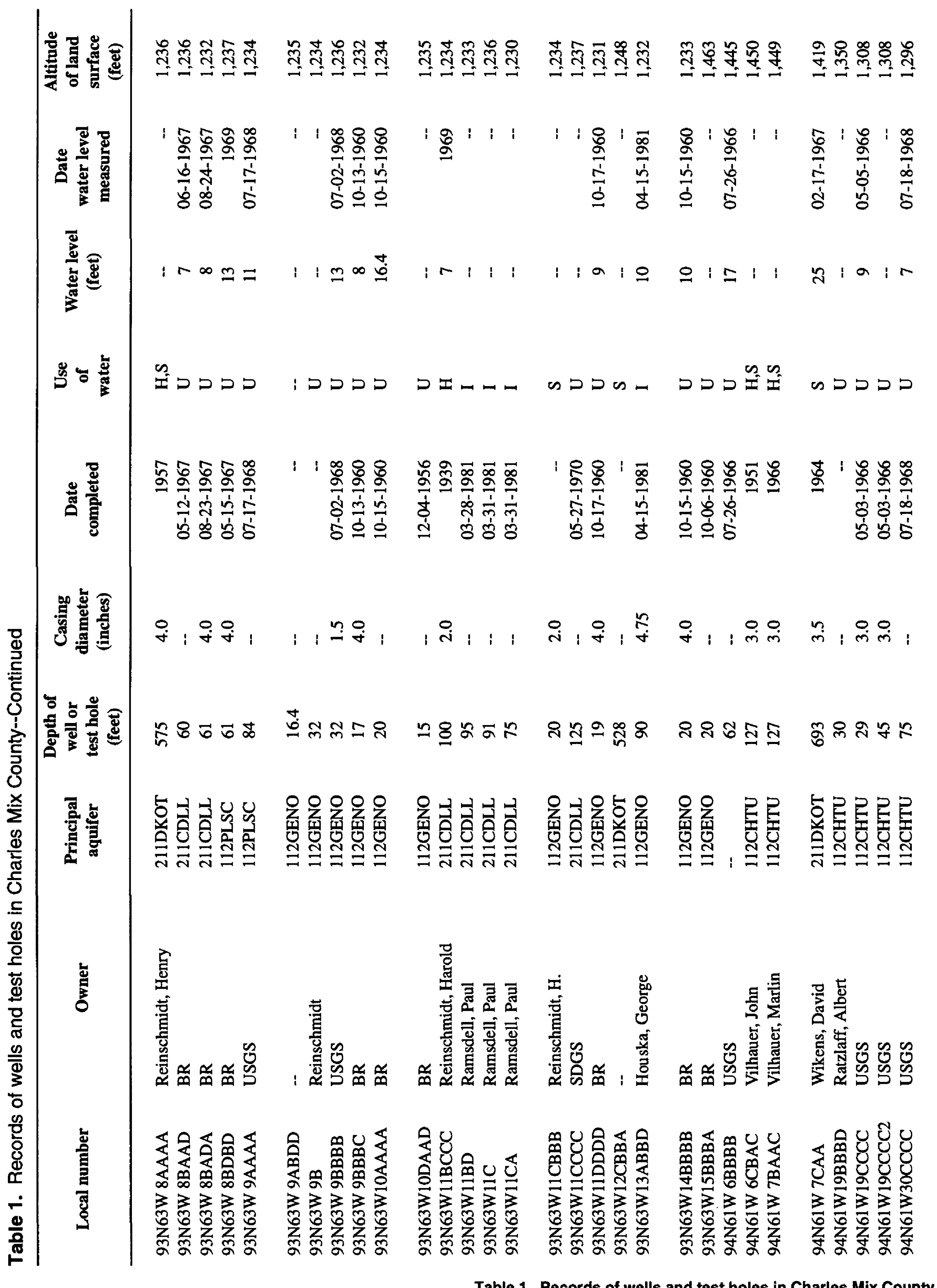

Table 1. Records of wells and test holes in Charles Mix County 


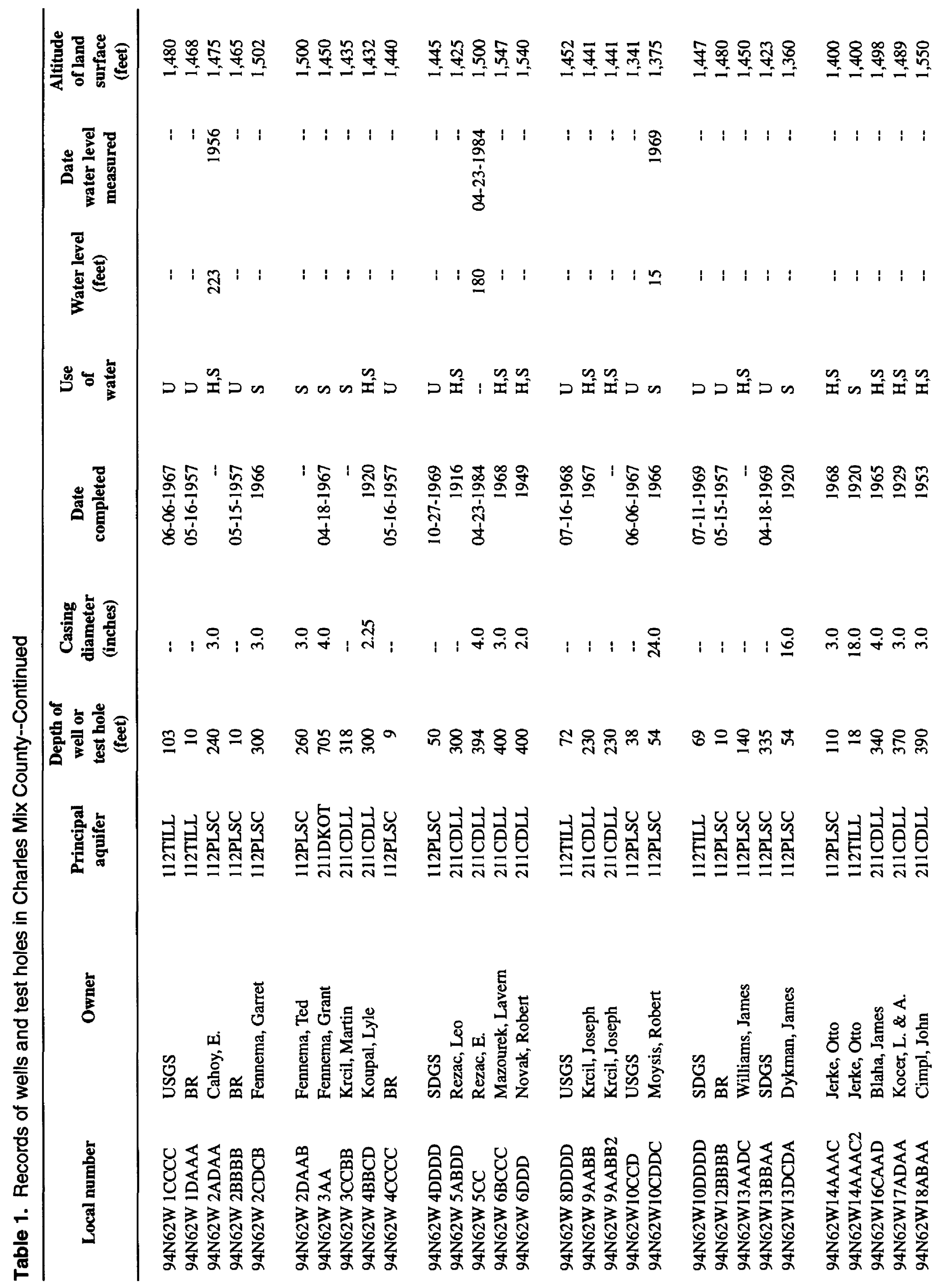

10 Records of Wells and Chemical Analyses of Ground Water in Charles Mix and Douglas Counties, South Dakota 


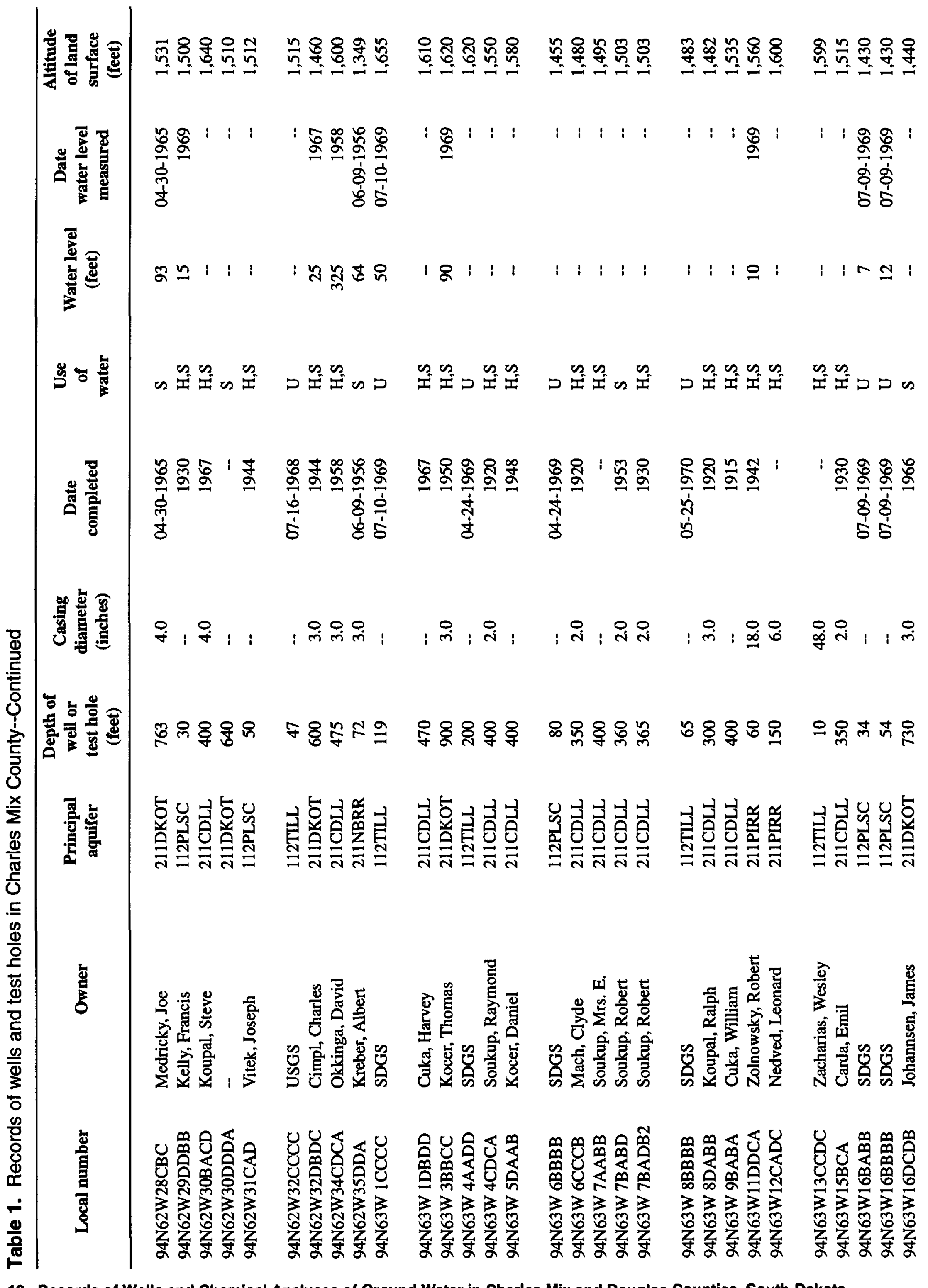

12 Records of Wells and Chemical Analyses of Ground Water in Charles Mix and Douglas Counties, South Dakota 


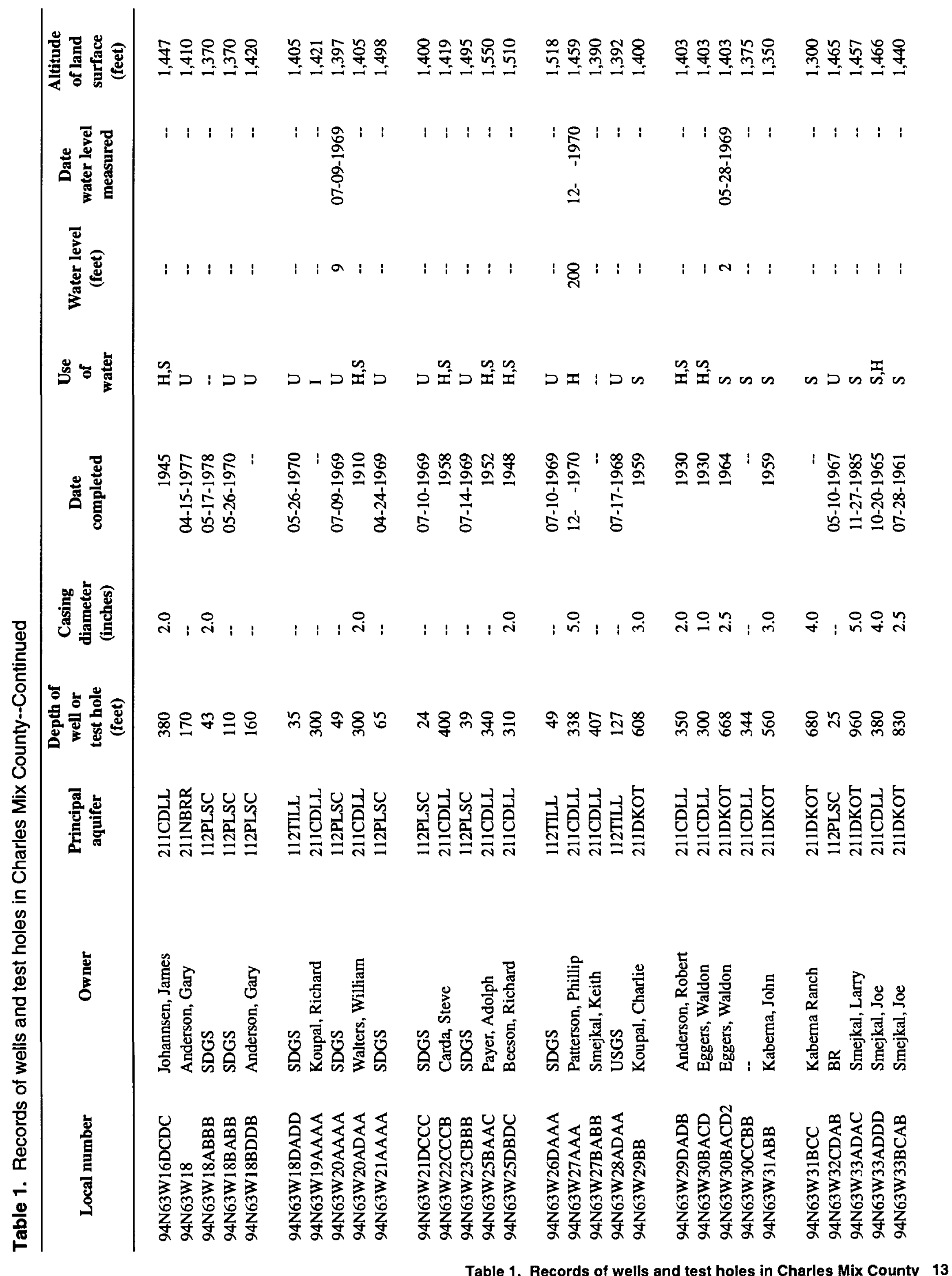




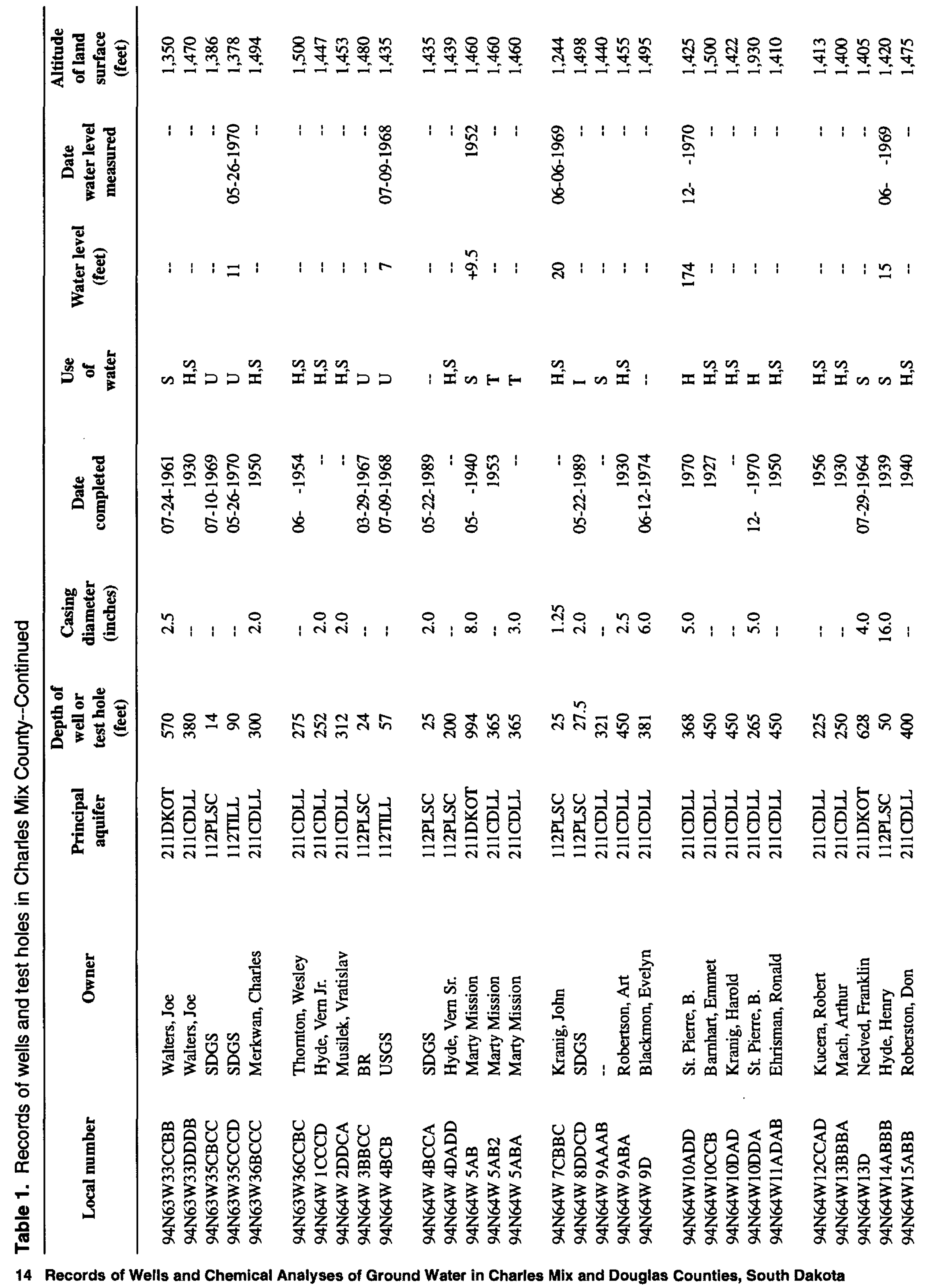




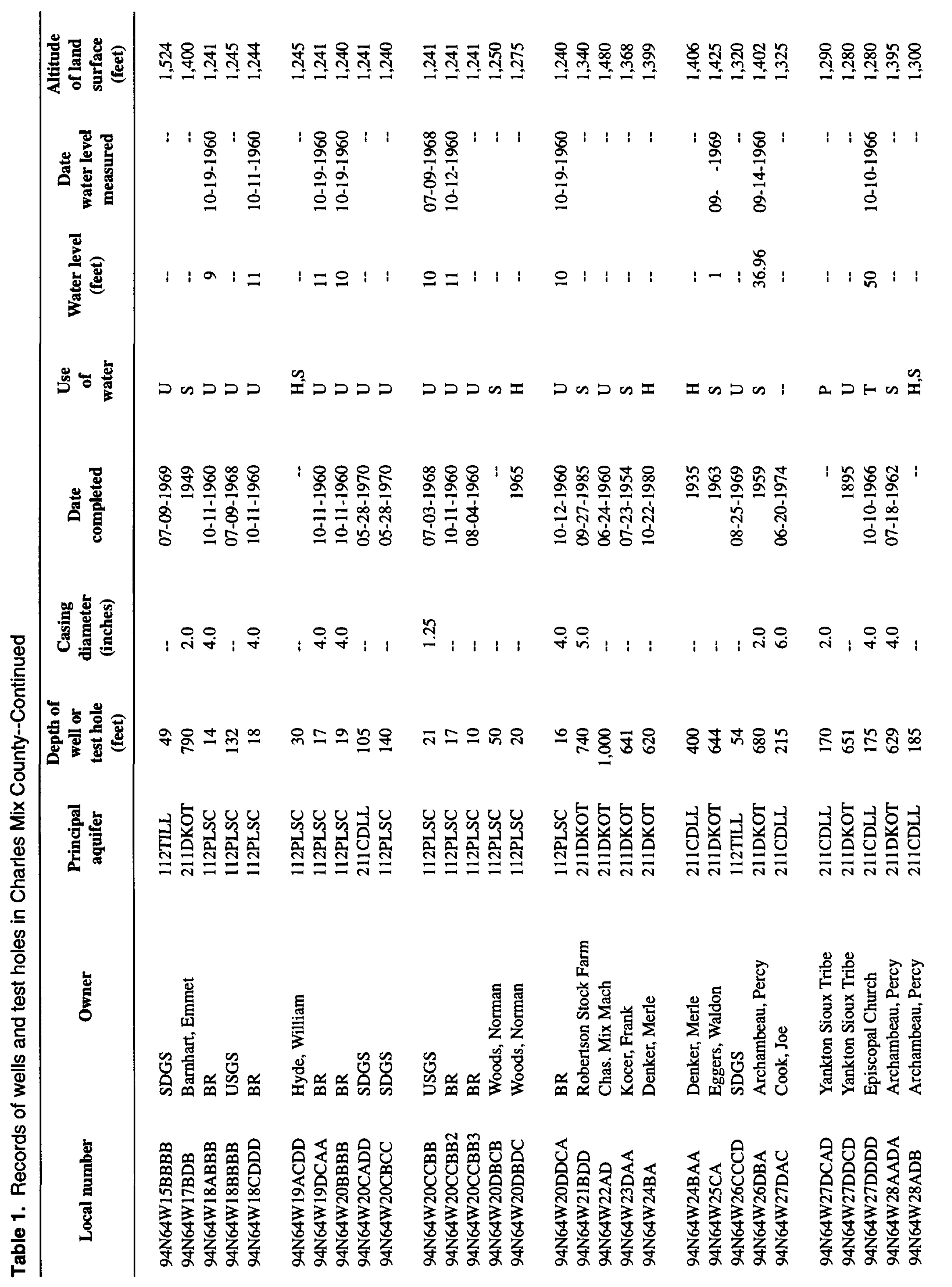




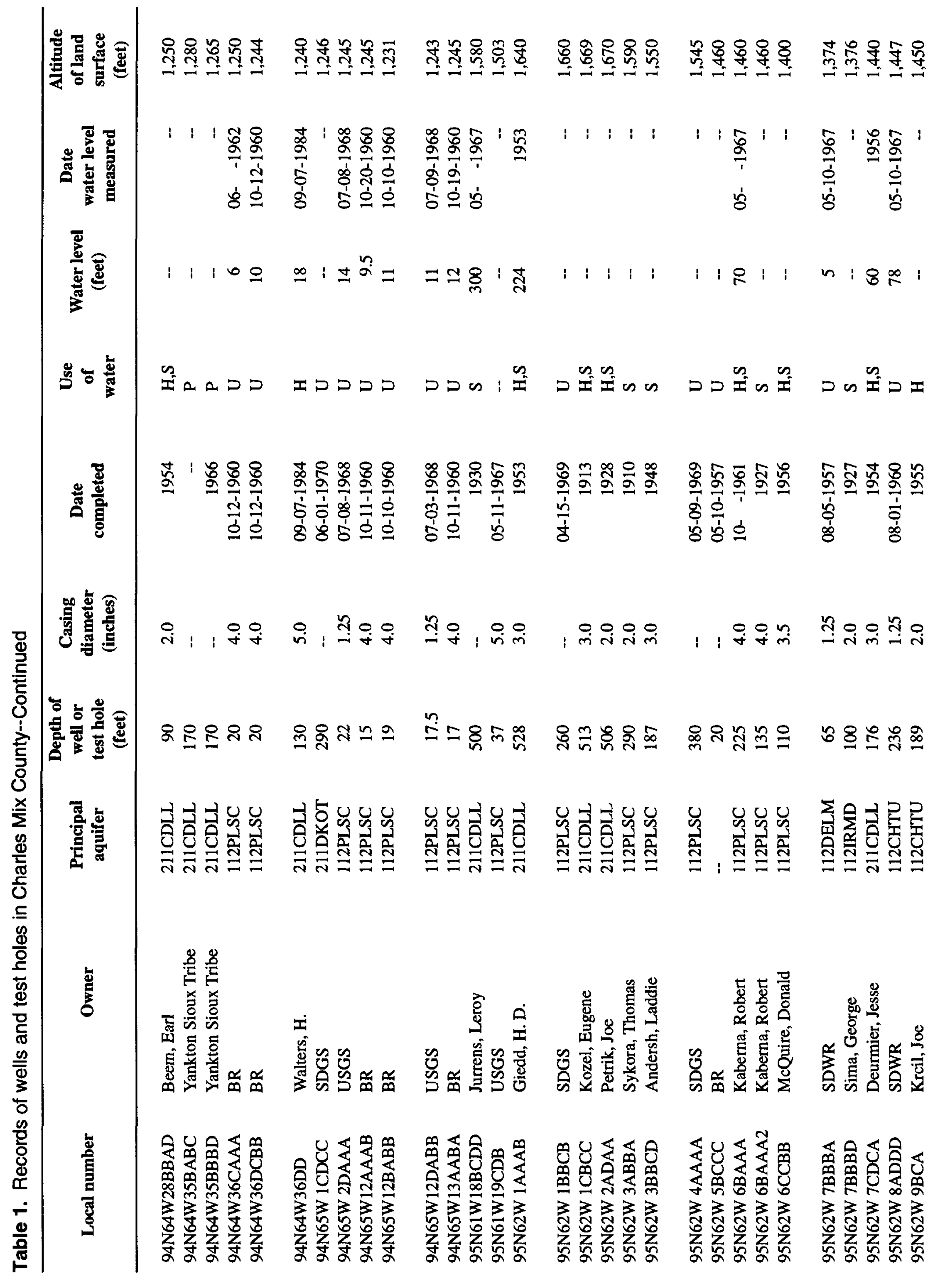

16 Records of Wells and Chemical Analyses of Ground Water in Charles Mix and Douglas Counties, South Dakota 


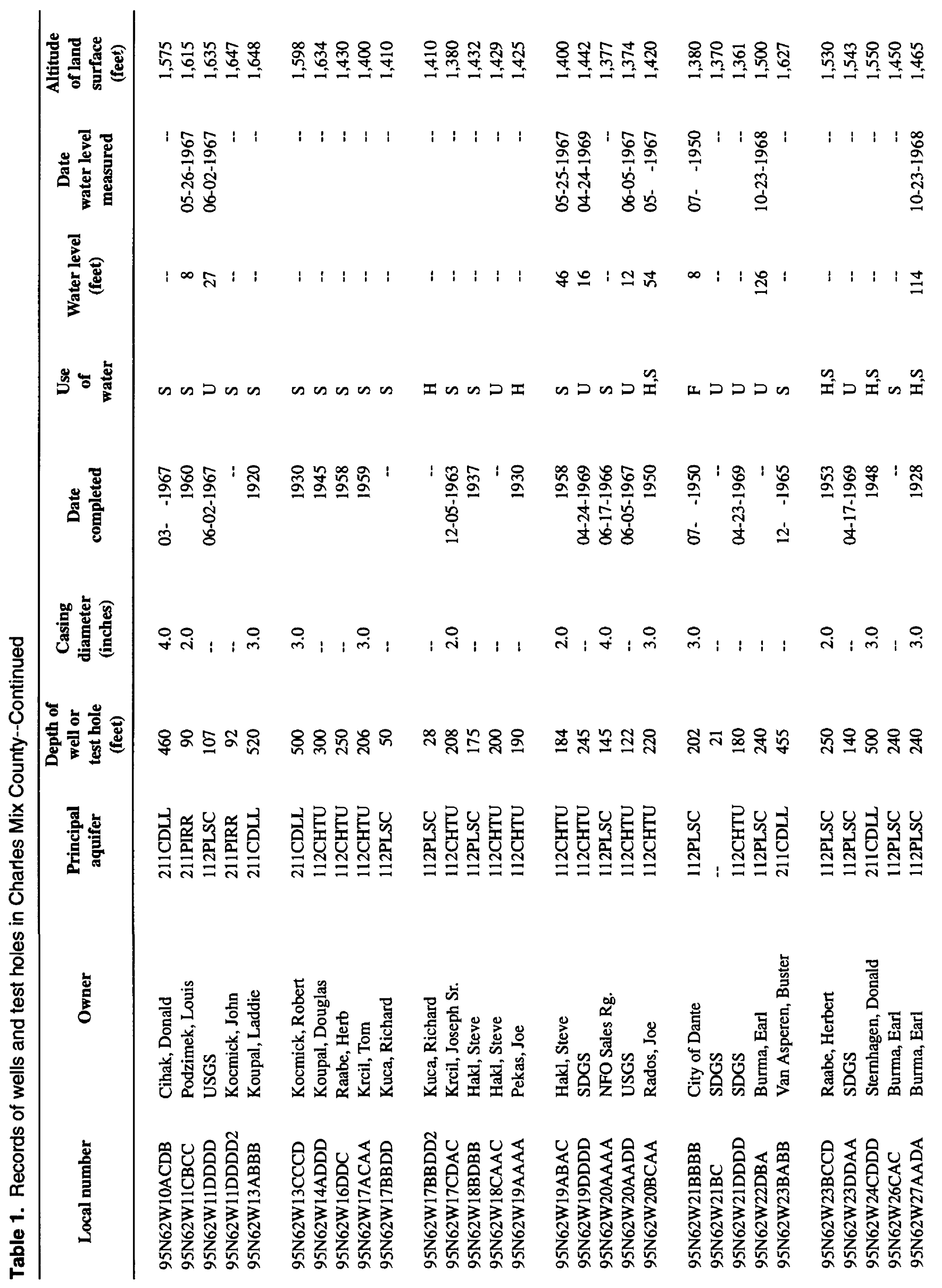




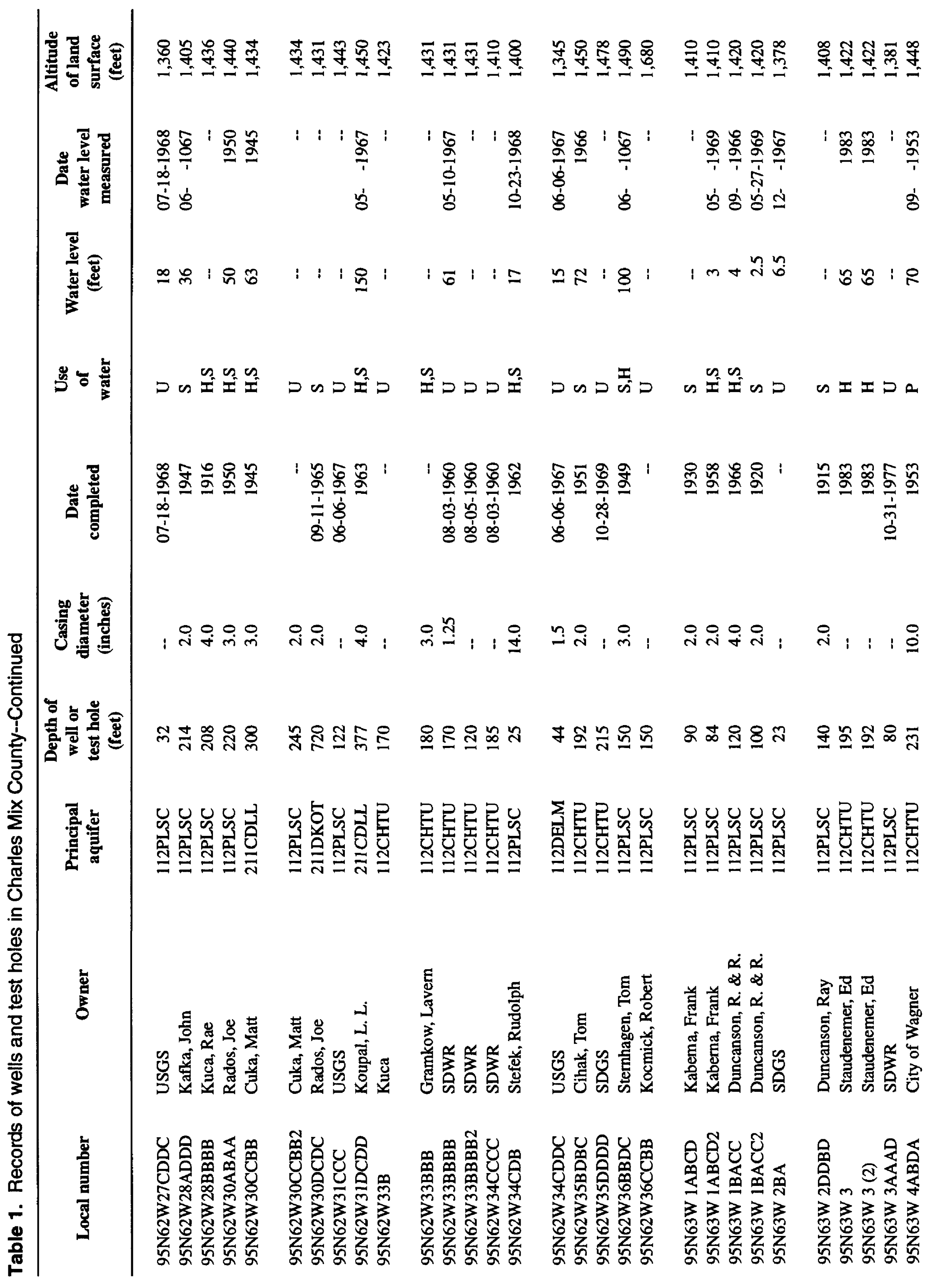

18 Records of Wells and Chemical Analyses of Ground Water in Charles Mix and Douglas Counties, South Dakota 


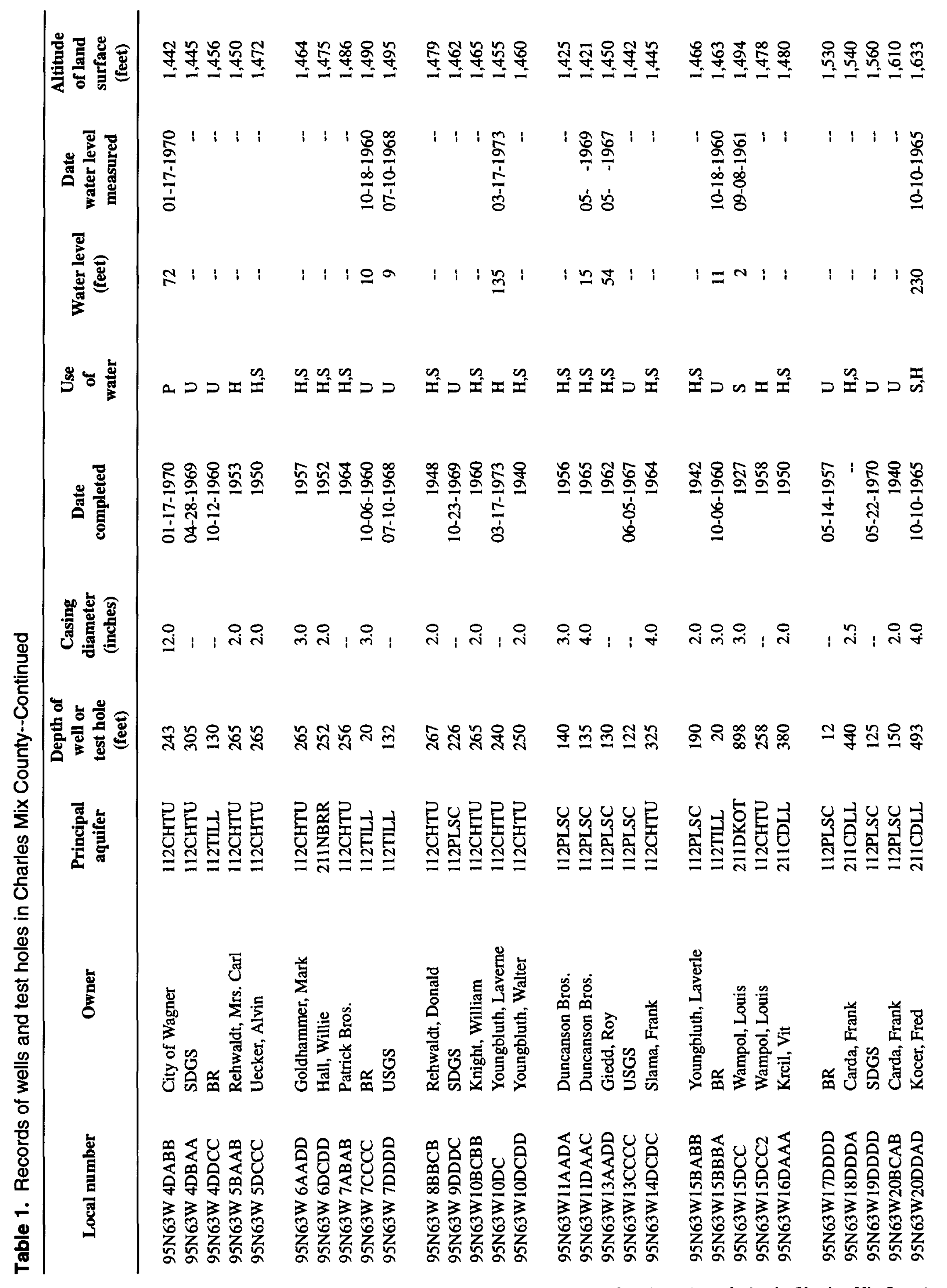

Tabla 1. Records of wells and test holes in Charles Mix County 19 


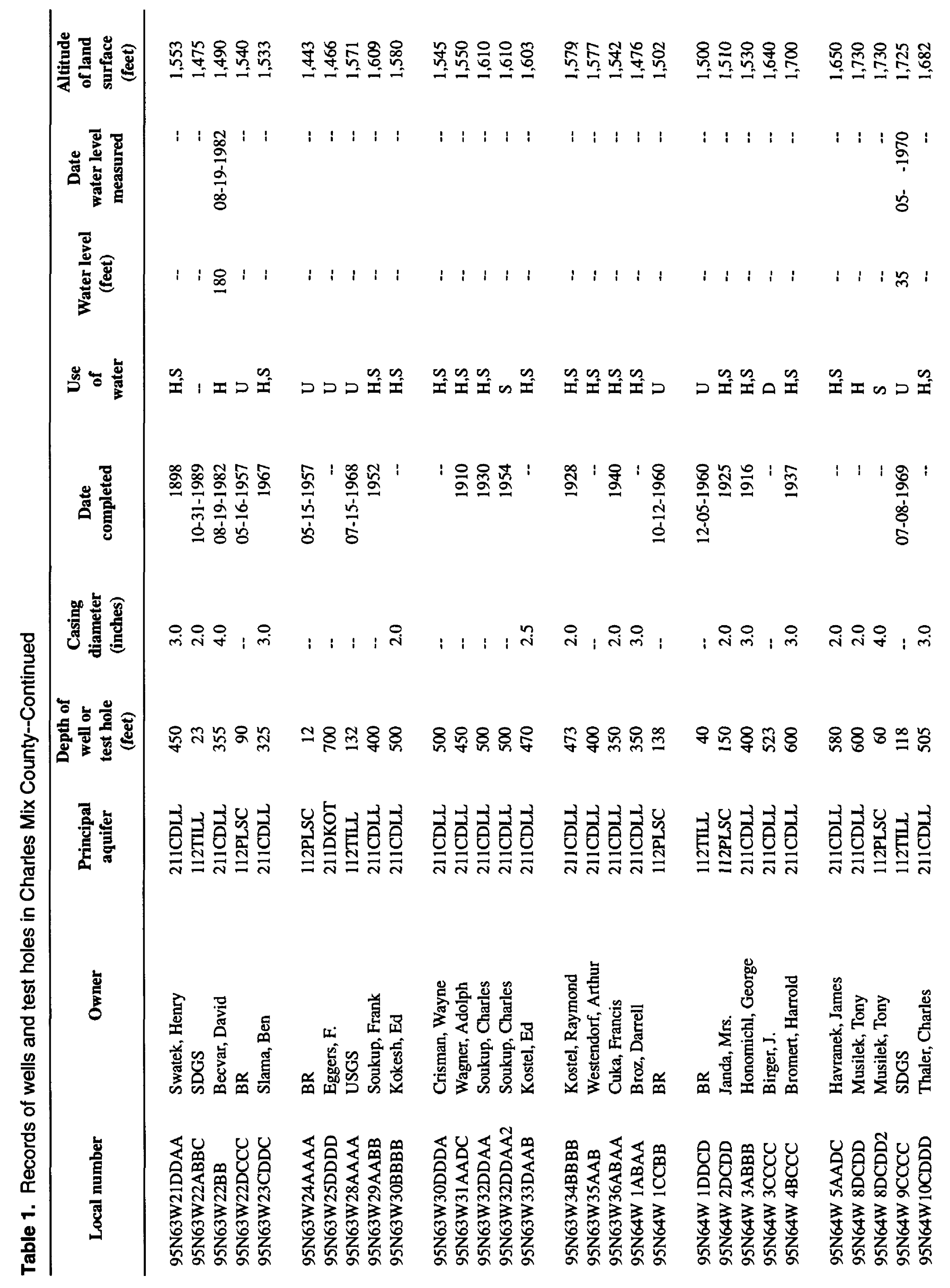

20 Records of Wells and Chemical Analyses of Ground Water in Charles Mix and Douglas Countiea, South Dakota 


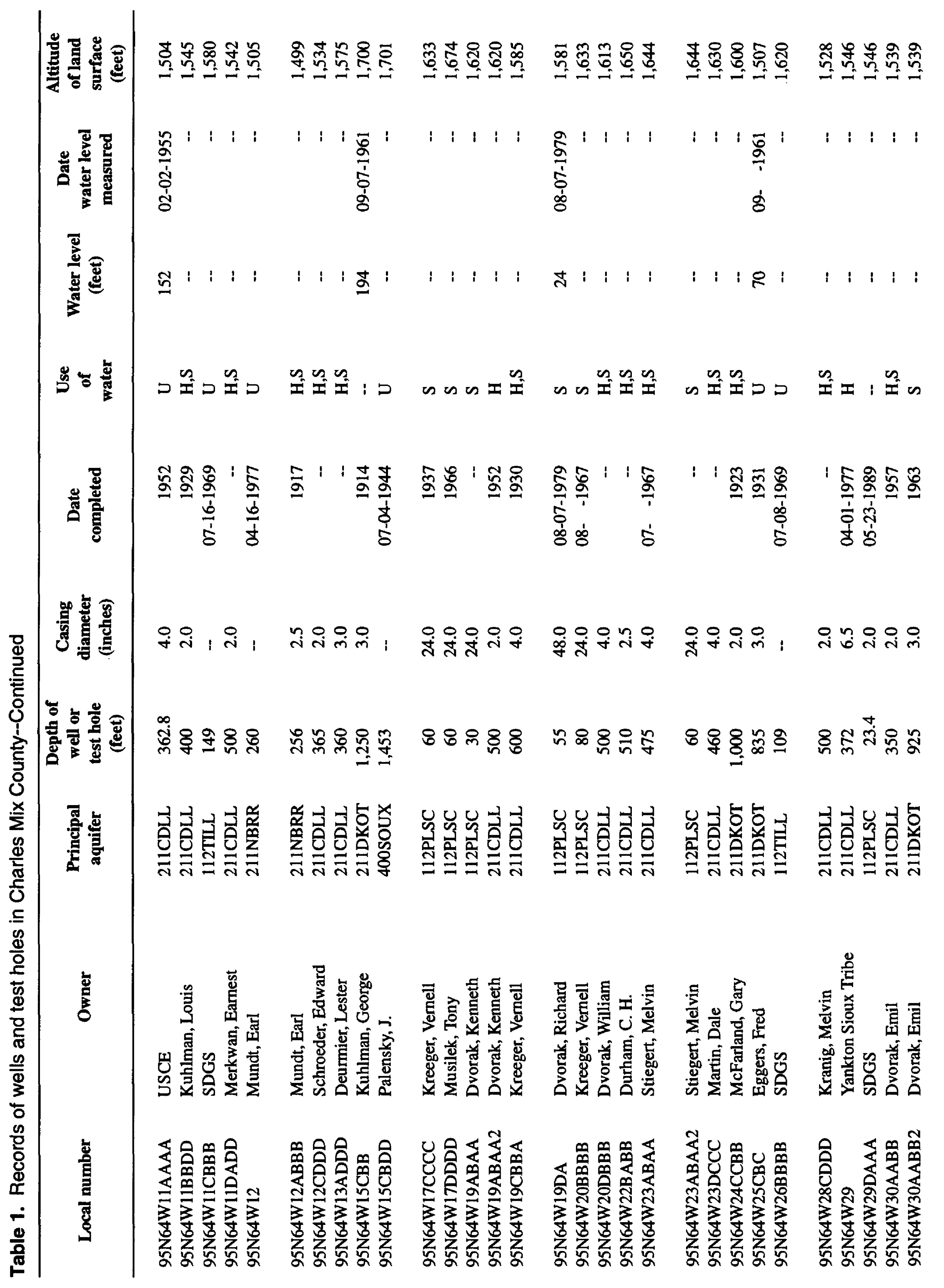




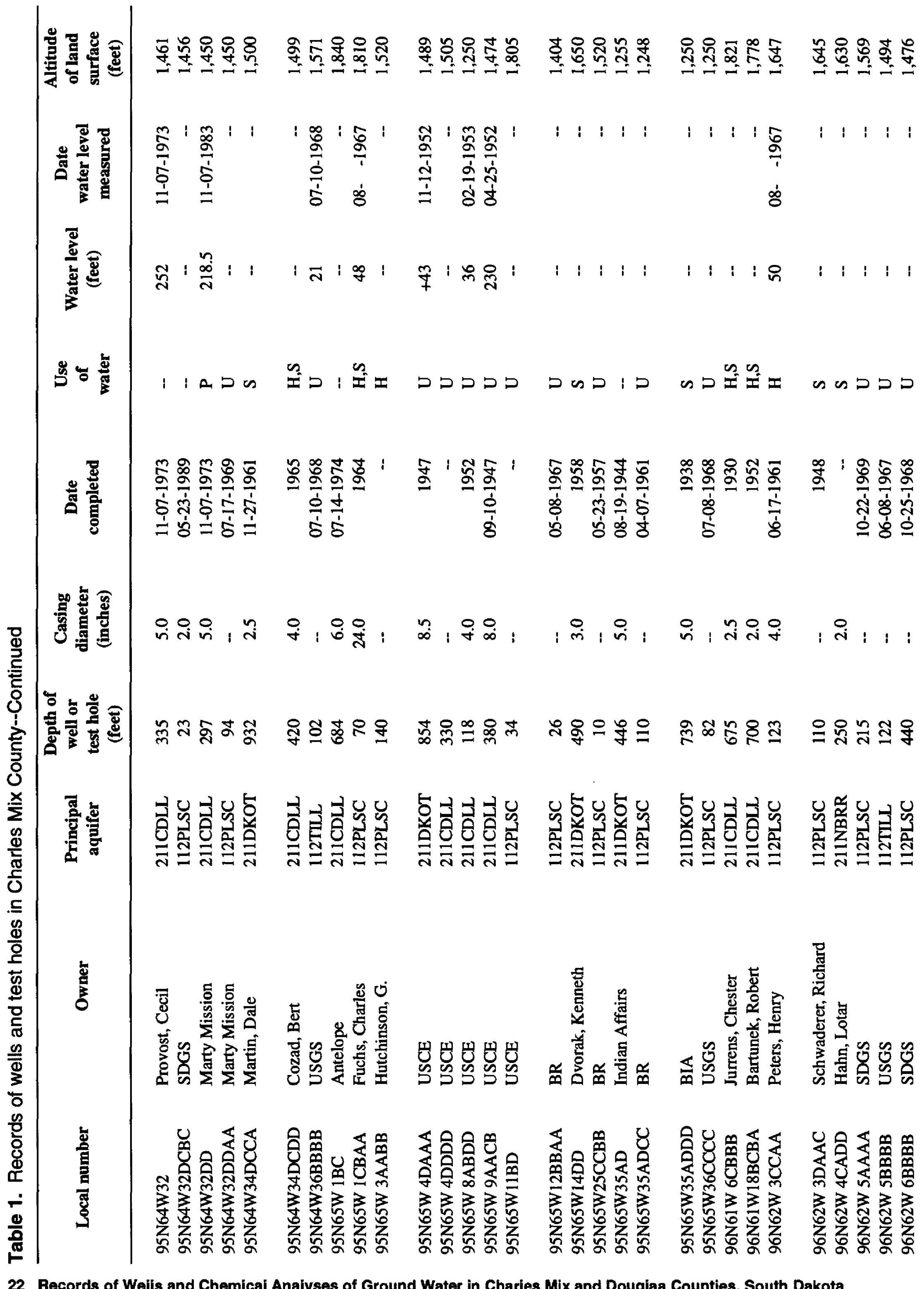




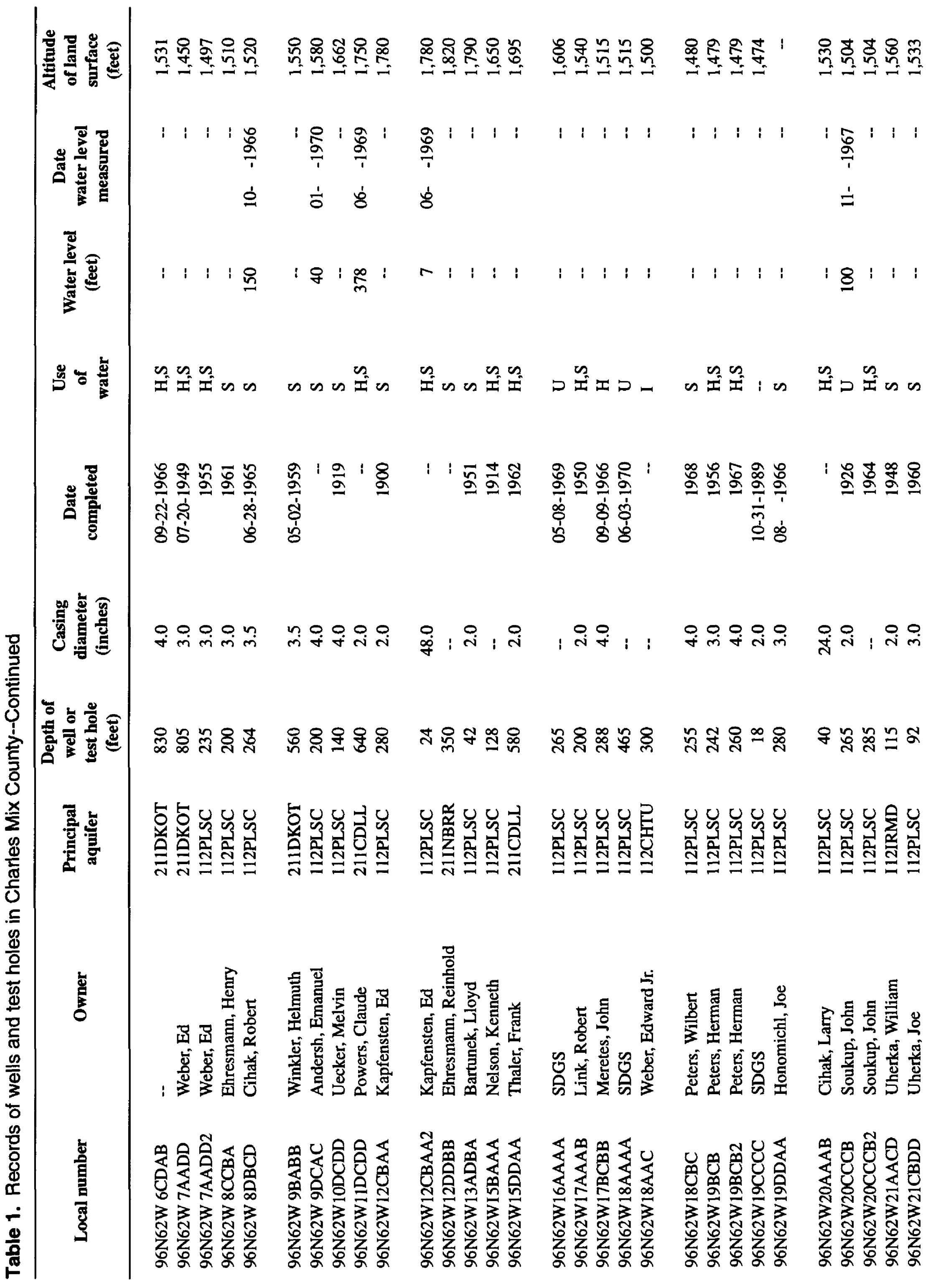




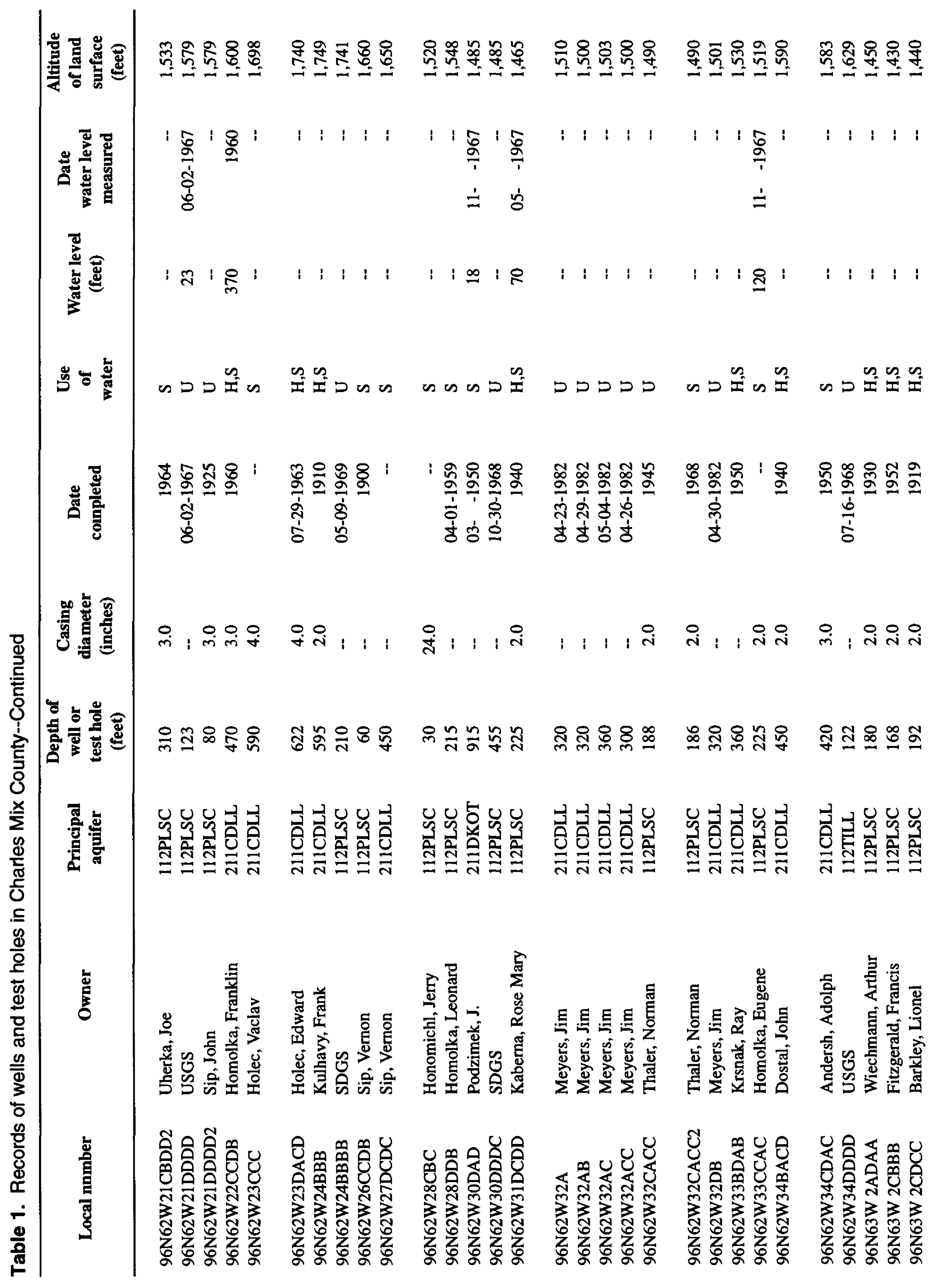




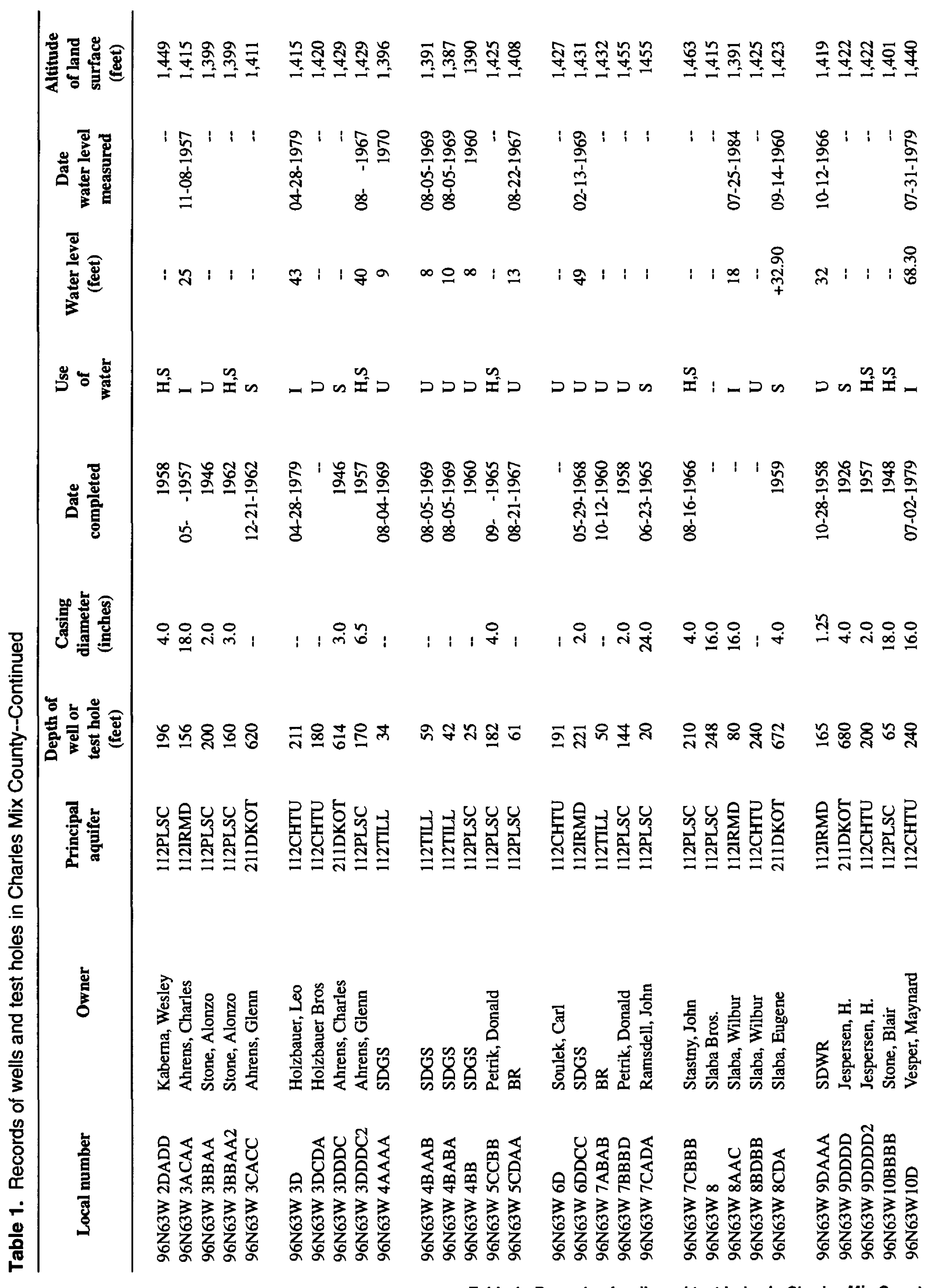

Tabla 1. Records of wells and tast holes in Charles Mix County 25 


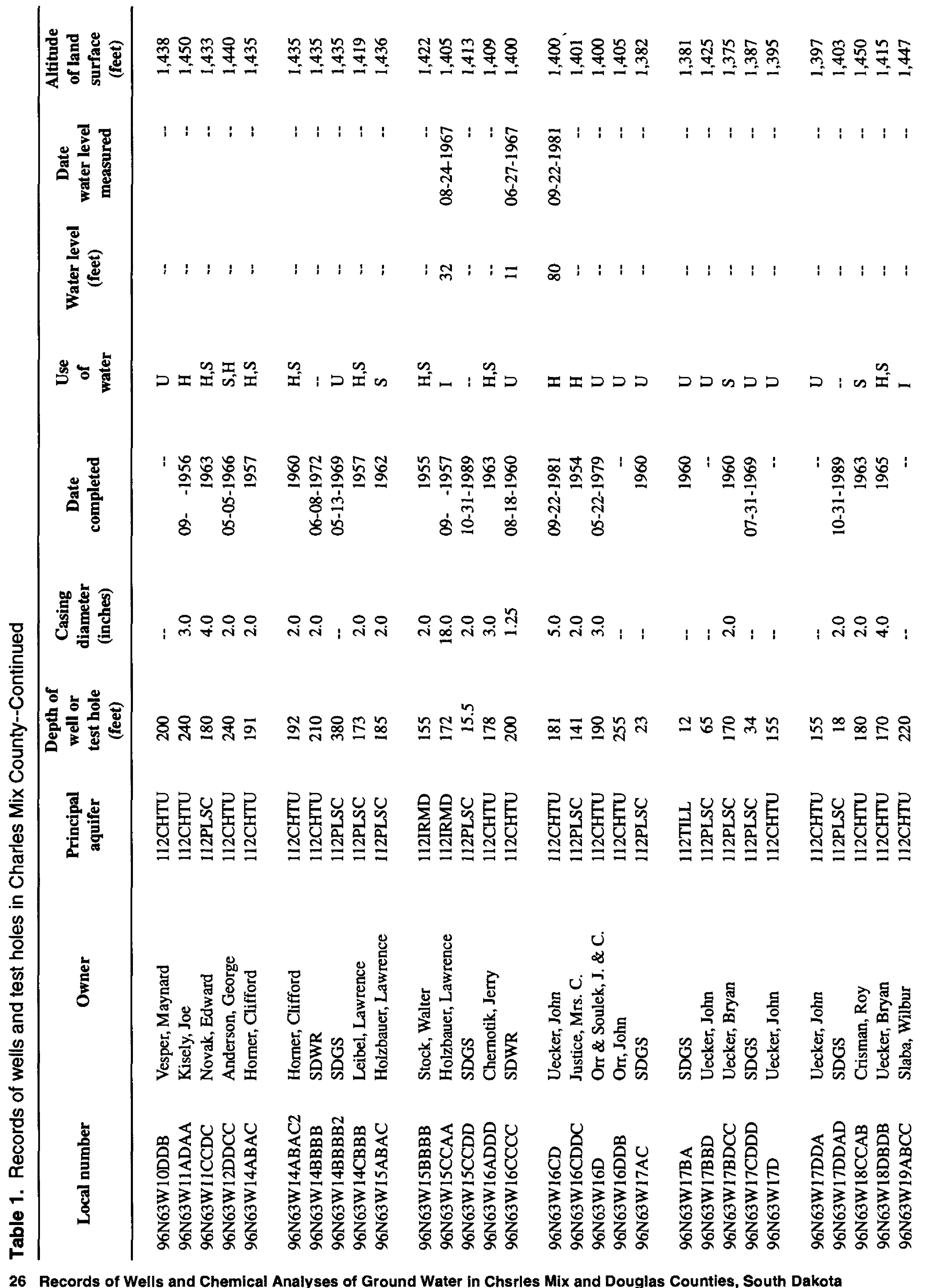




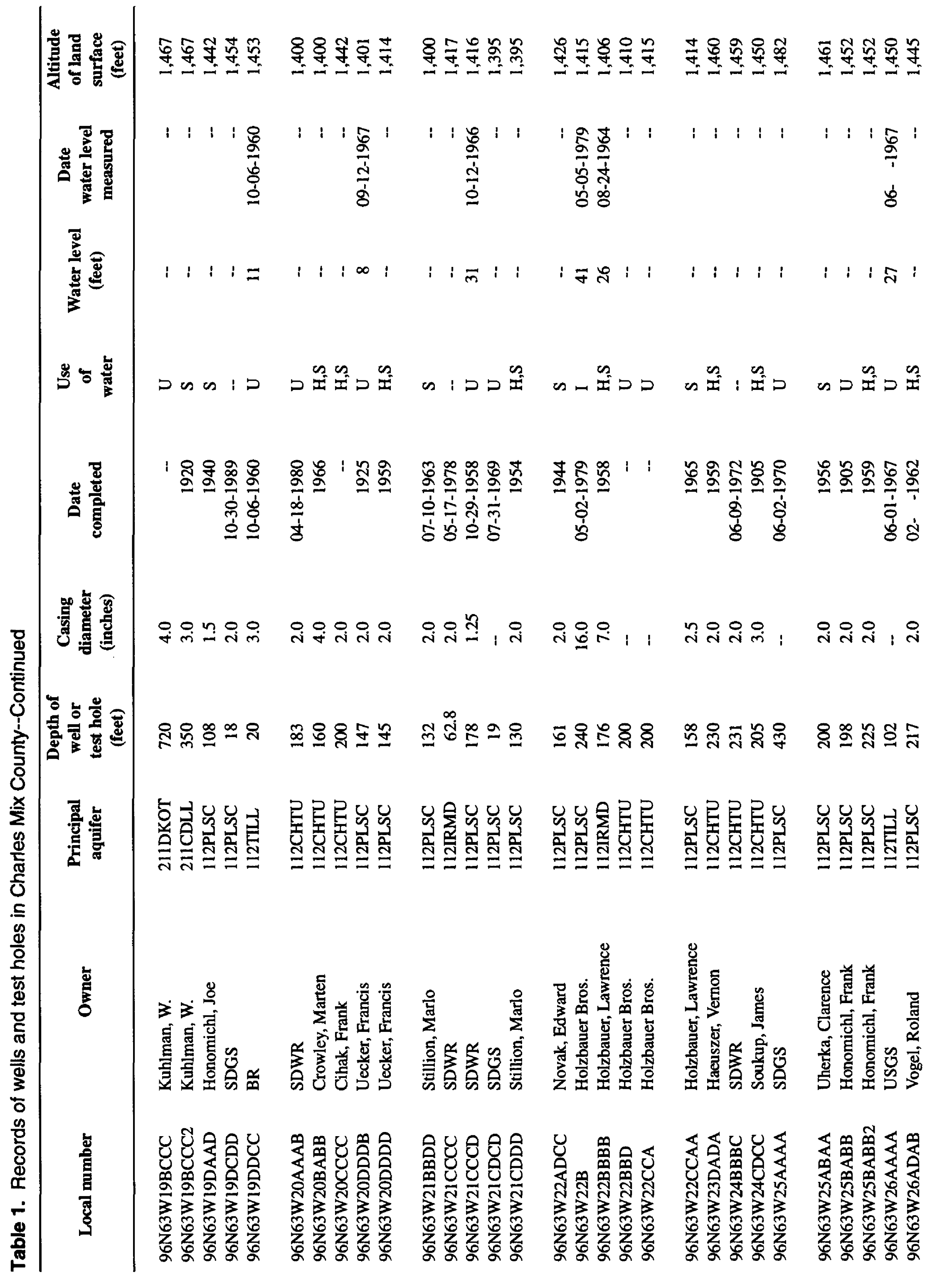




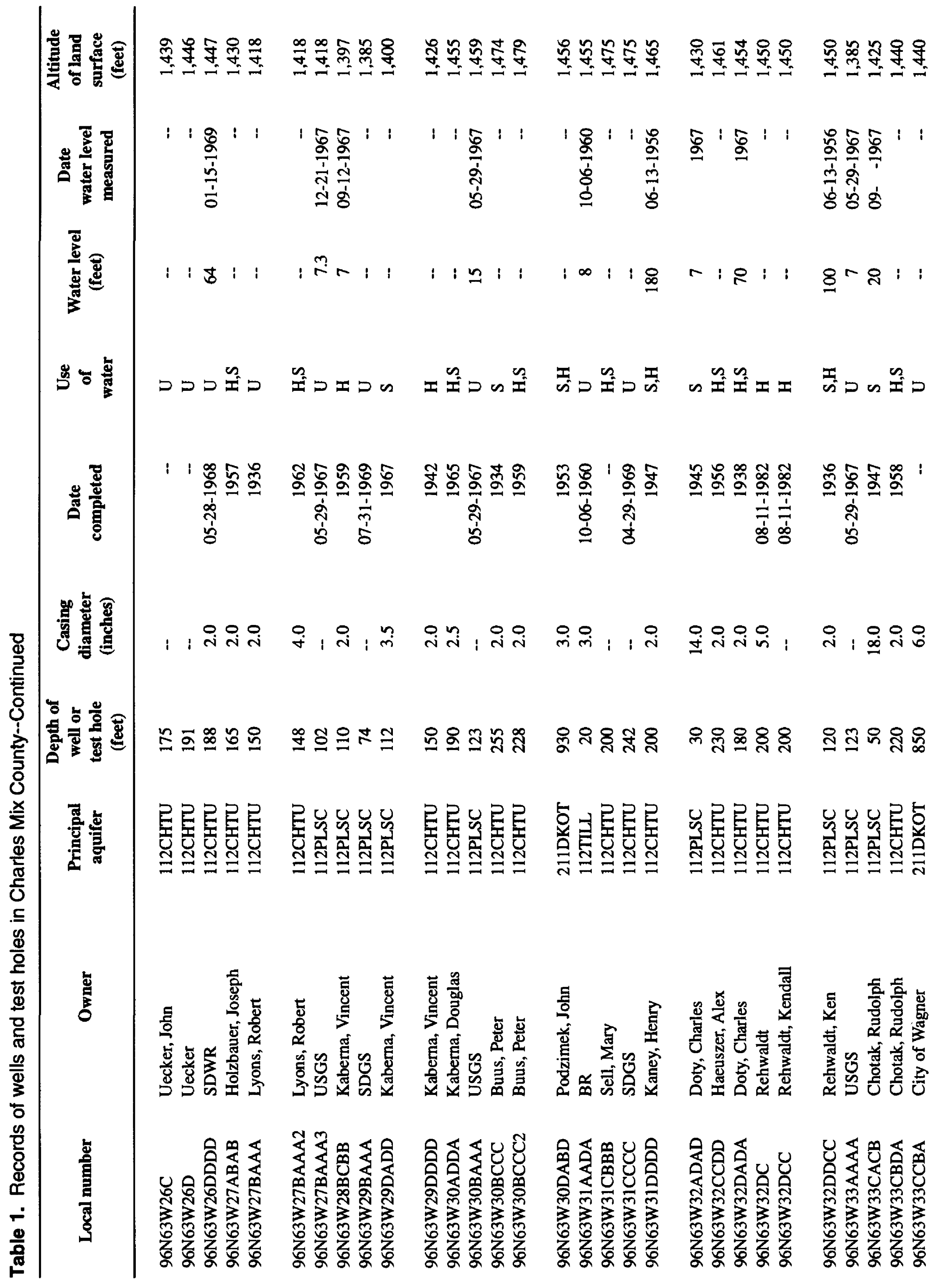

28 Records of Wells and Chemical Analyses of Ground Water in Charles Mix and Douglas Counties, South Dakota 


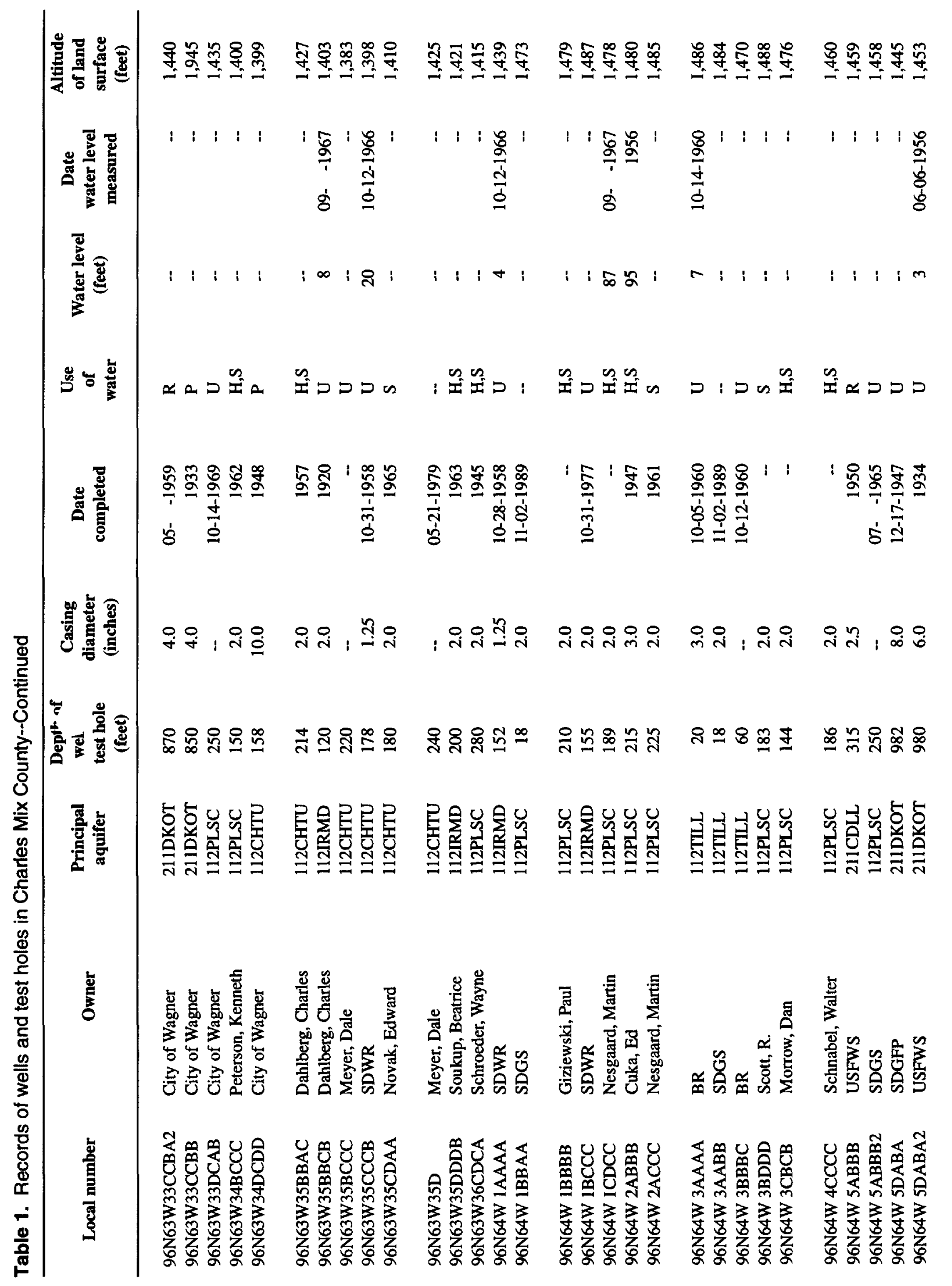




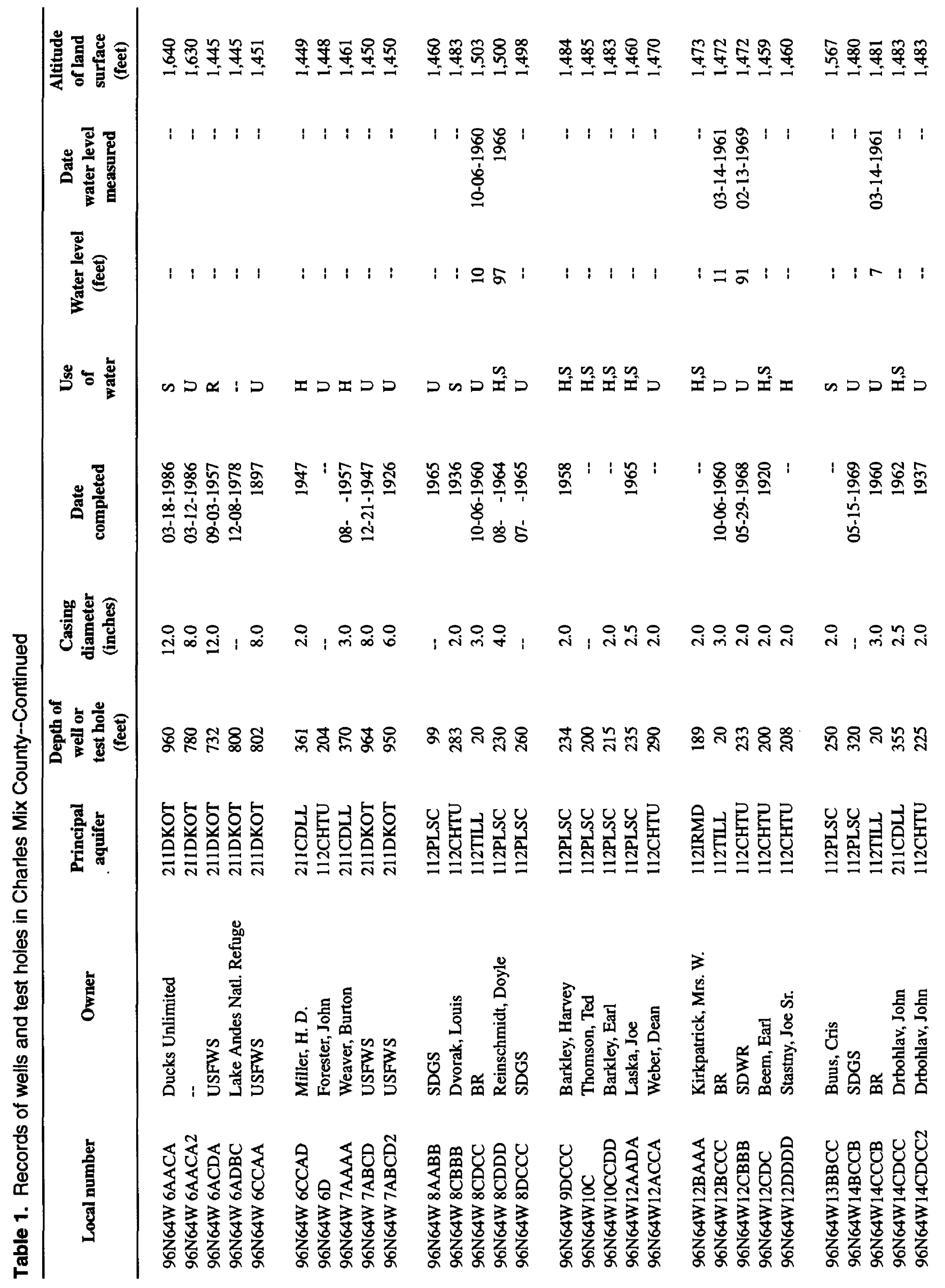

30 Records of Wells and Chemlcal Analyses of Ground Water in Charles Mix and Douglas Counties, South Dakota 


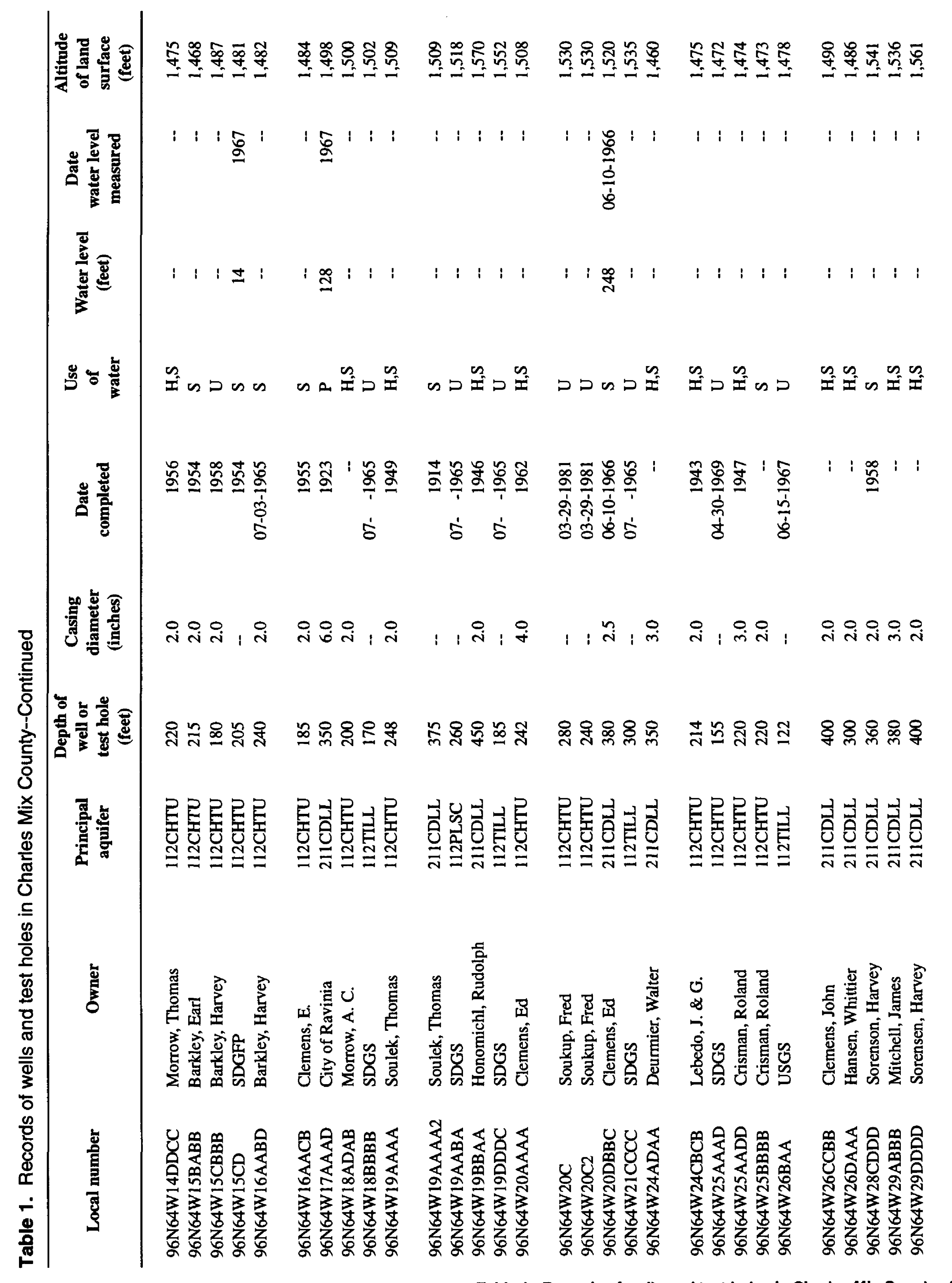

Table 1. Records of wells and test holes in Charles Mix County 31 


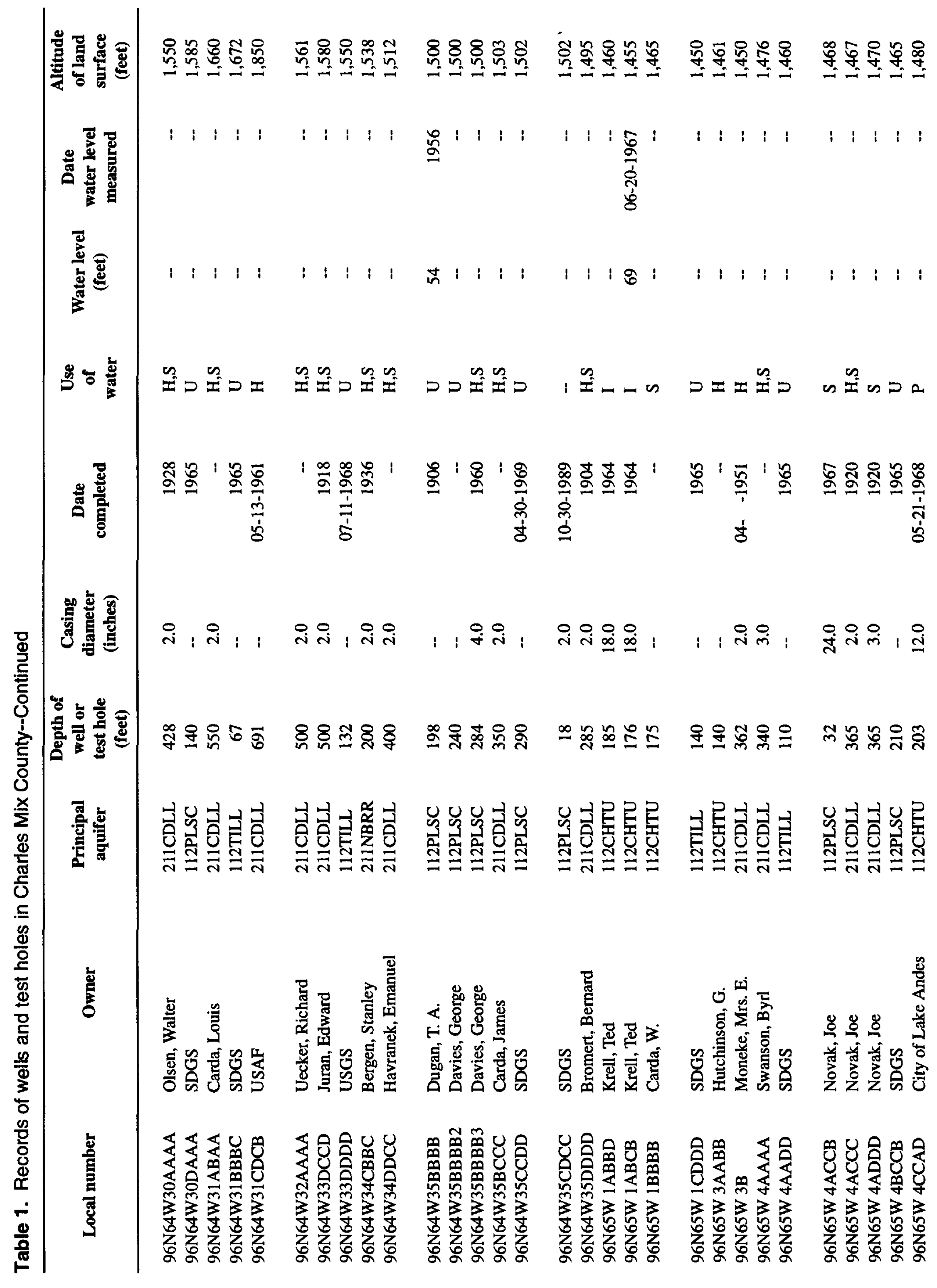

32 Records of Wells and Chemical Anslyses of Ground Water In Charles Mix and Douglas Counties, South Dakota 


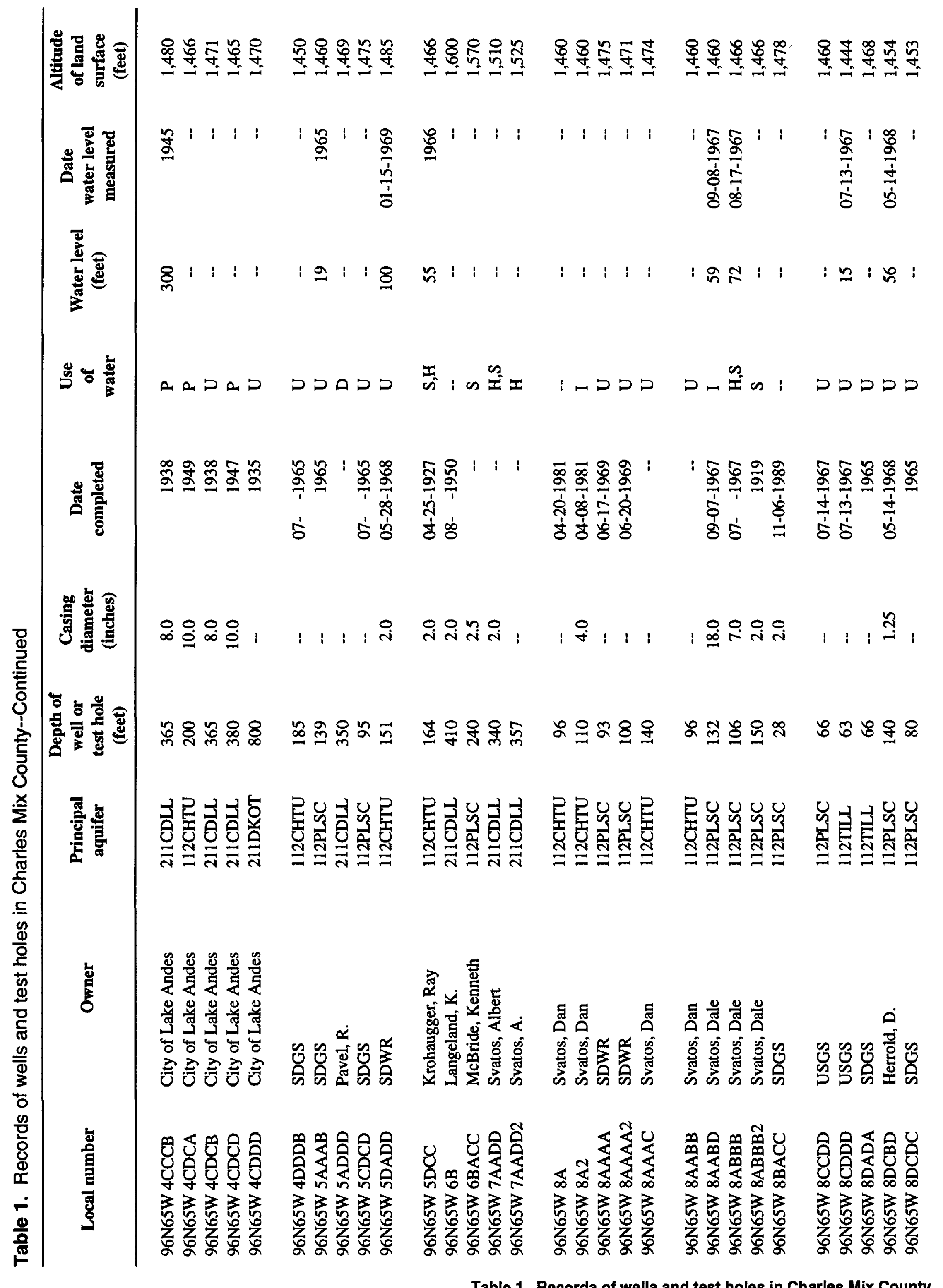




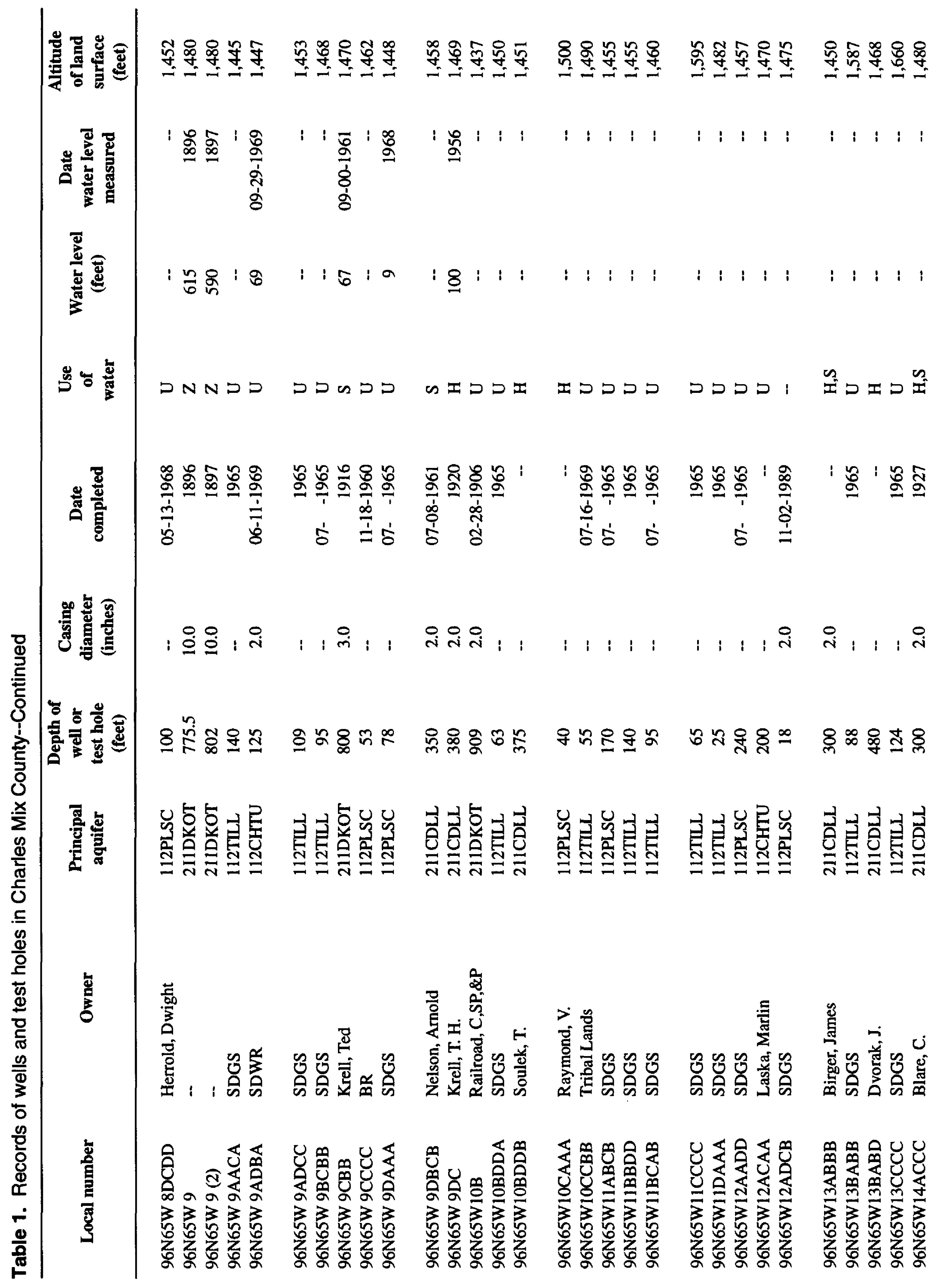

34 Records of Wells and Chemlcal Analyses of Ground Water in Charles Mix and Douglas Counties, South Dakota 


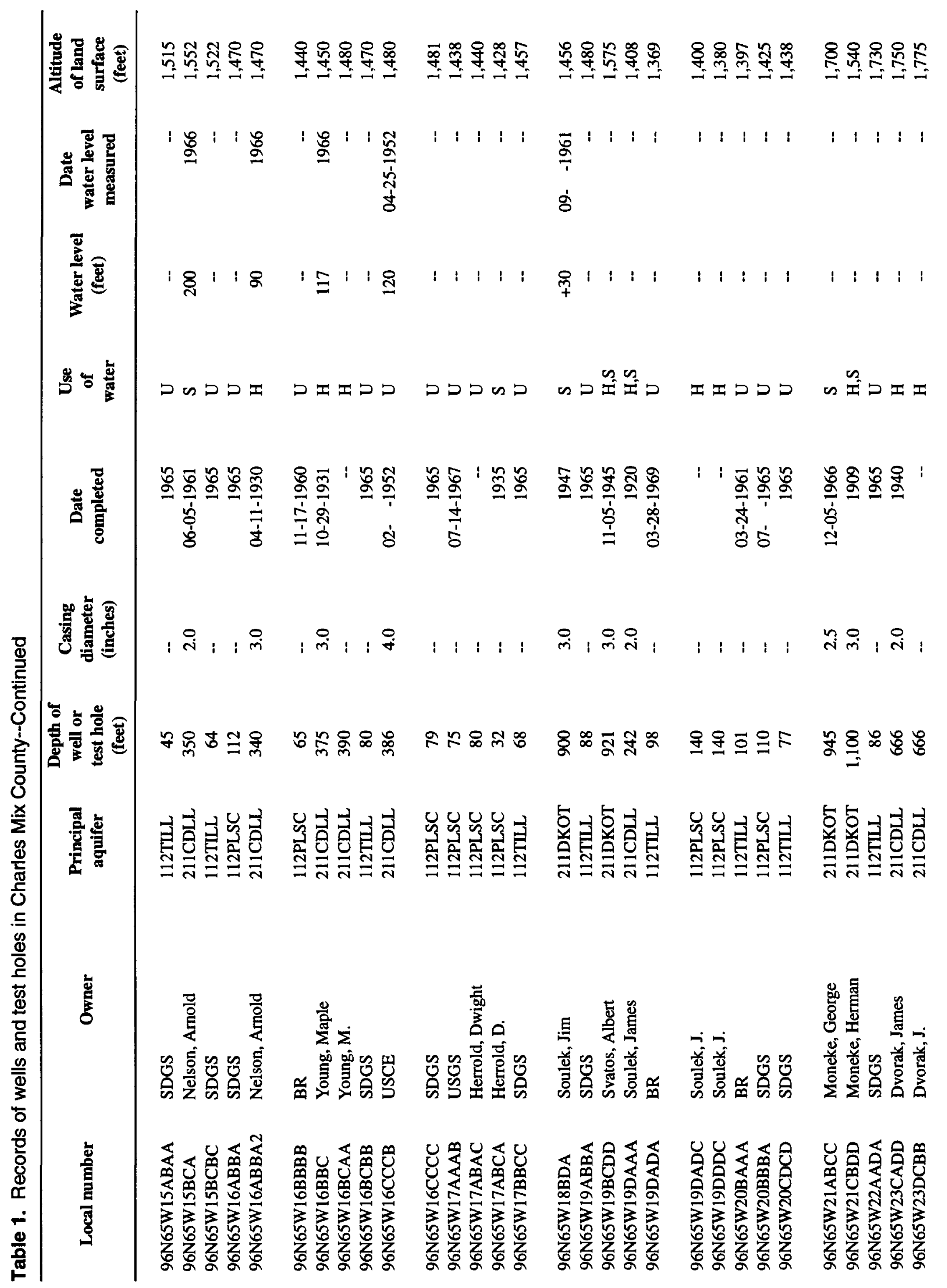

Tabla 1. Records of wella and test holea in Charles Mix County 35 


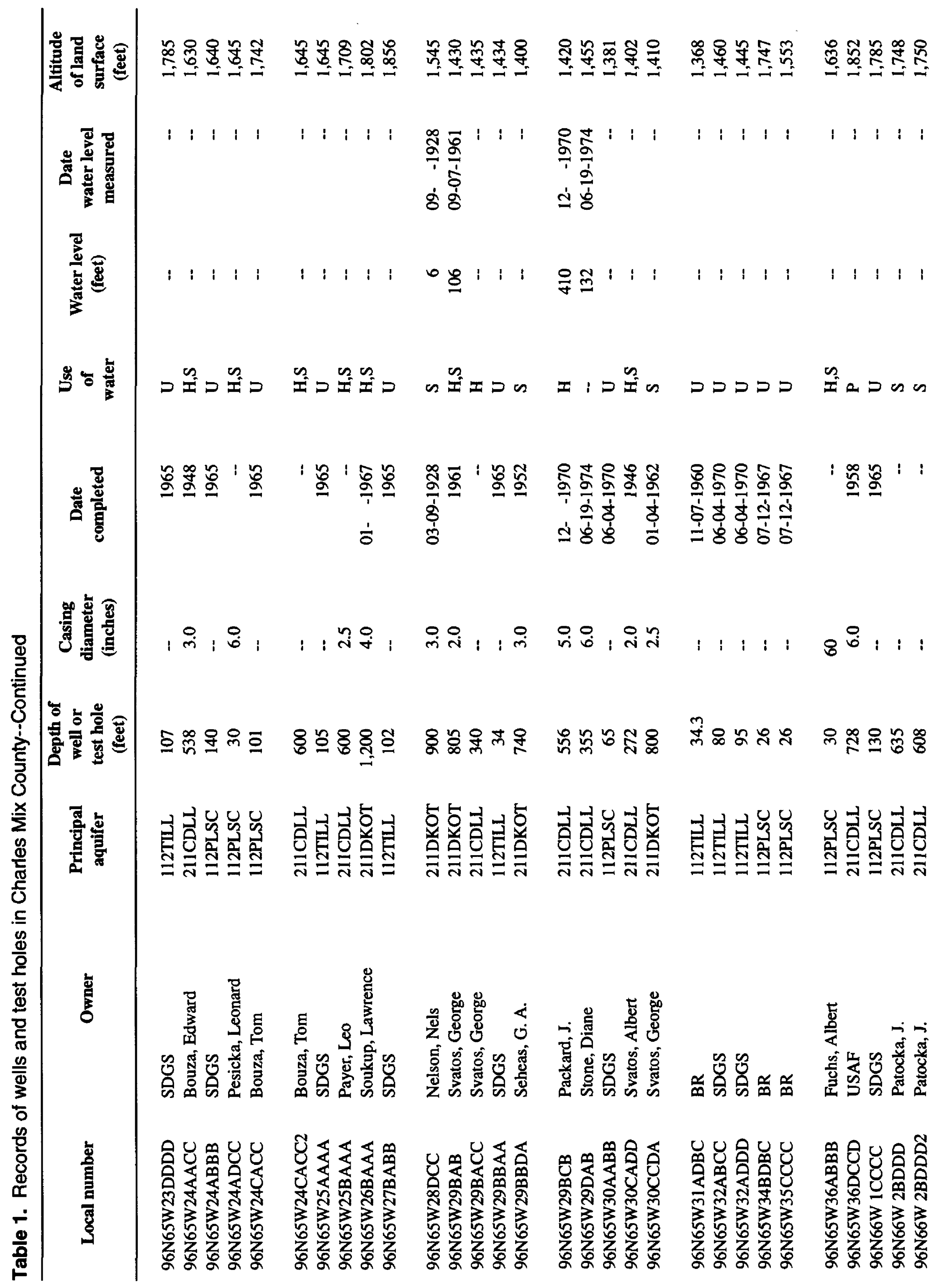




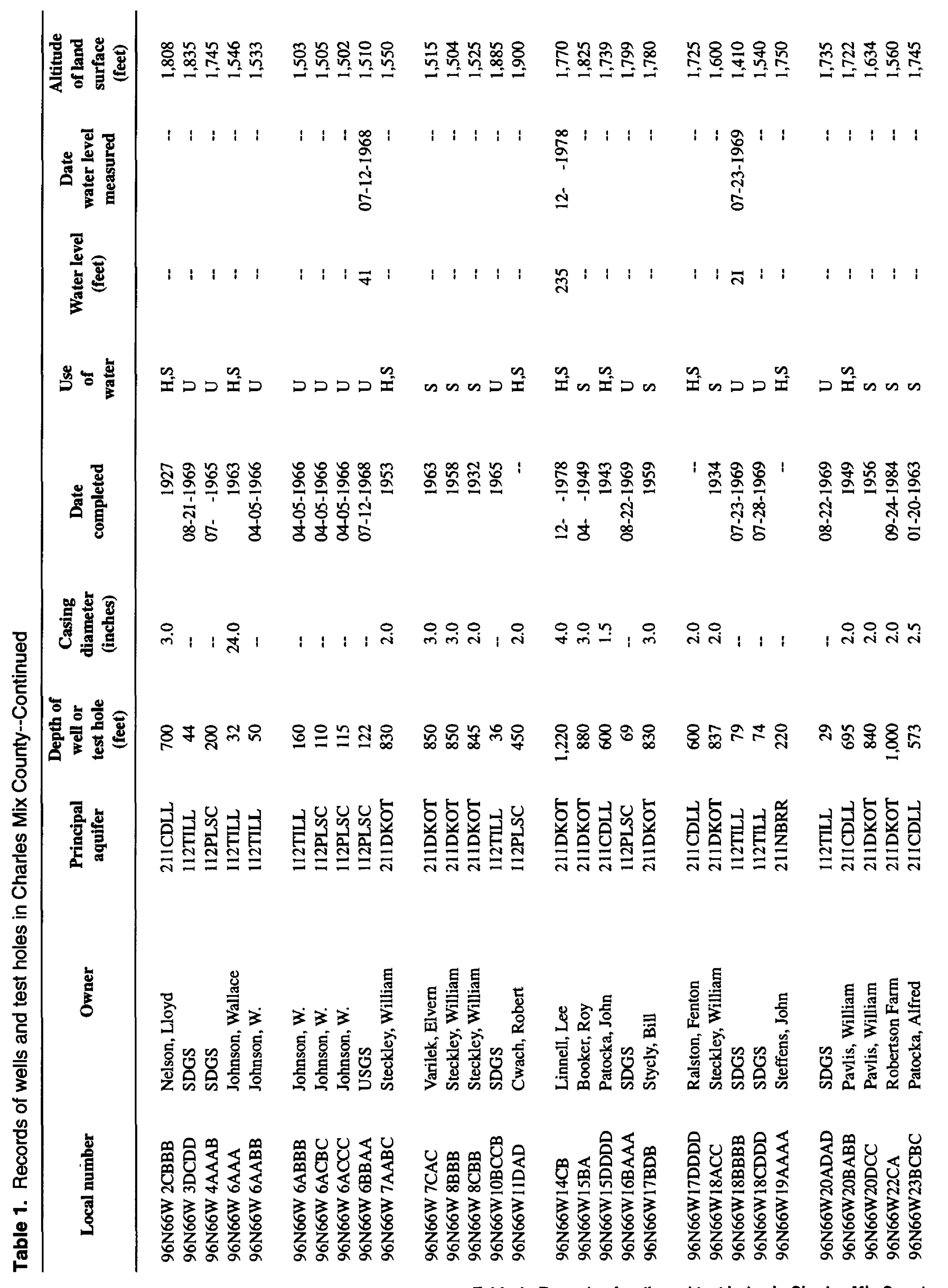




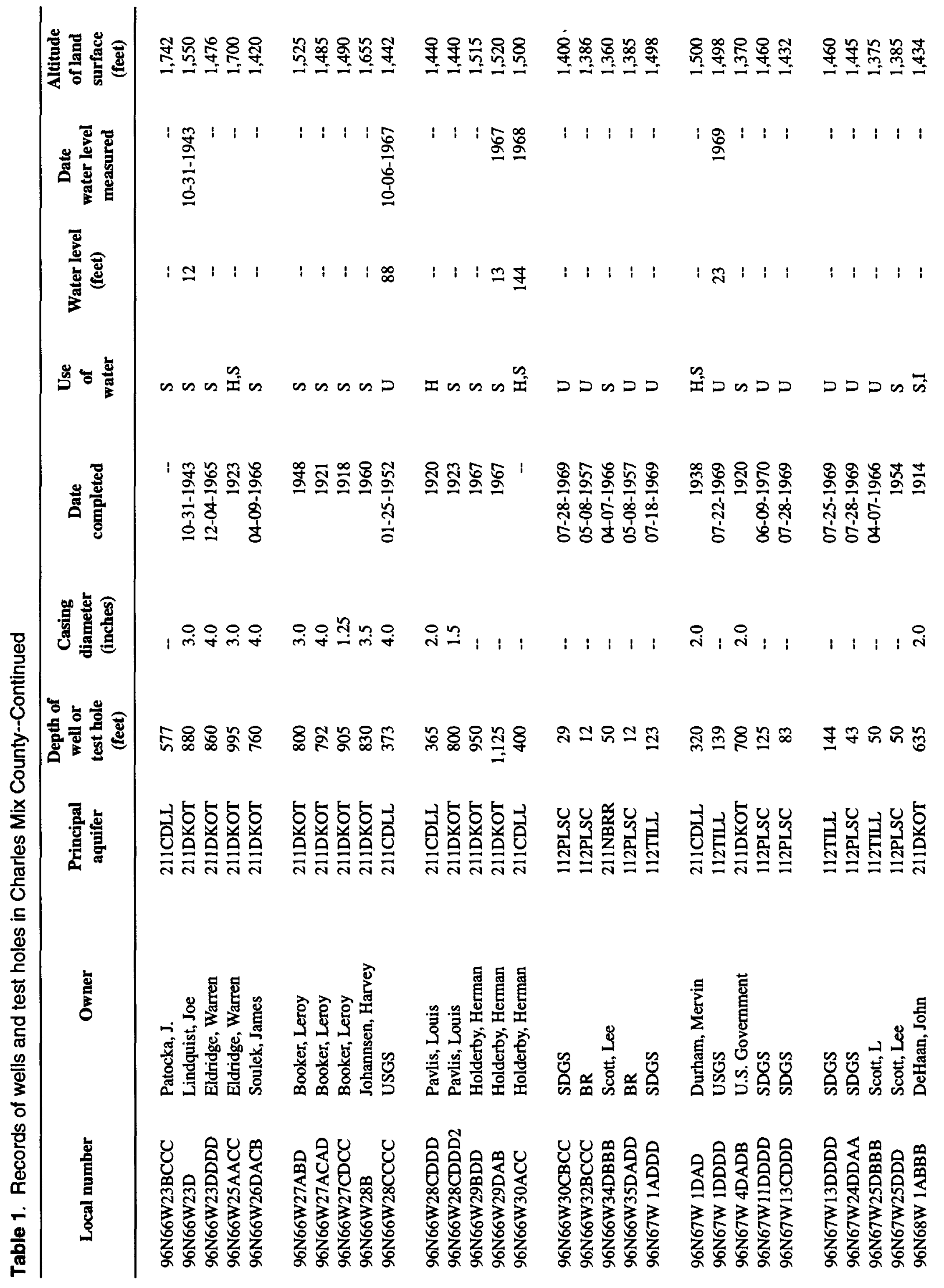




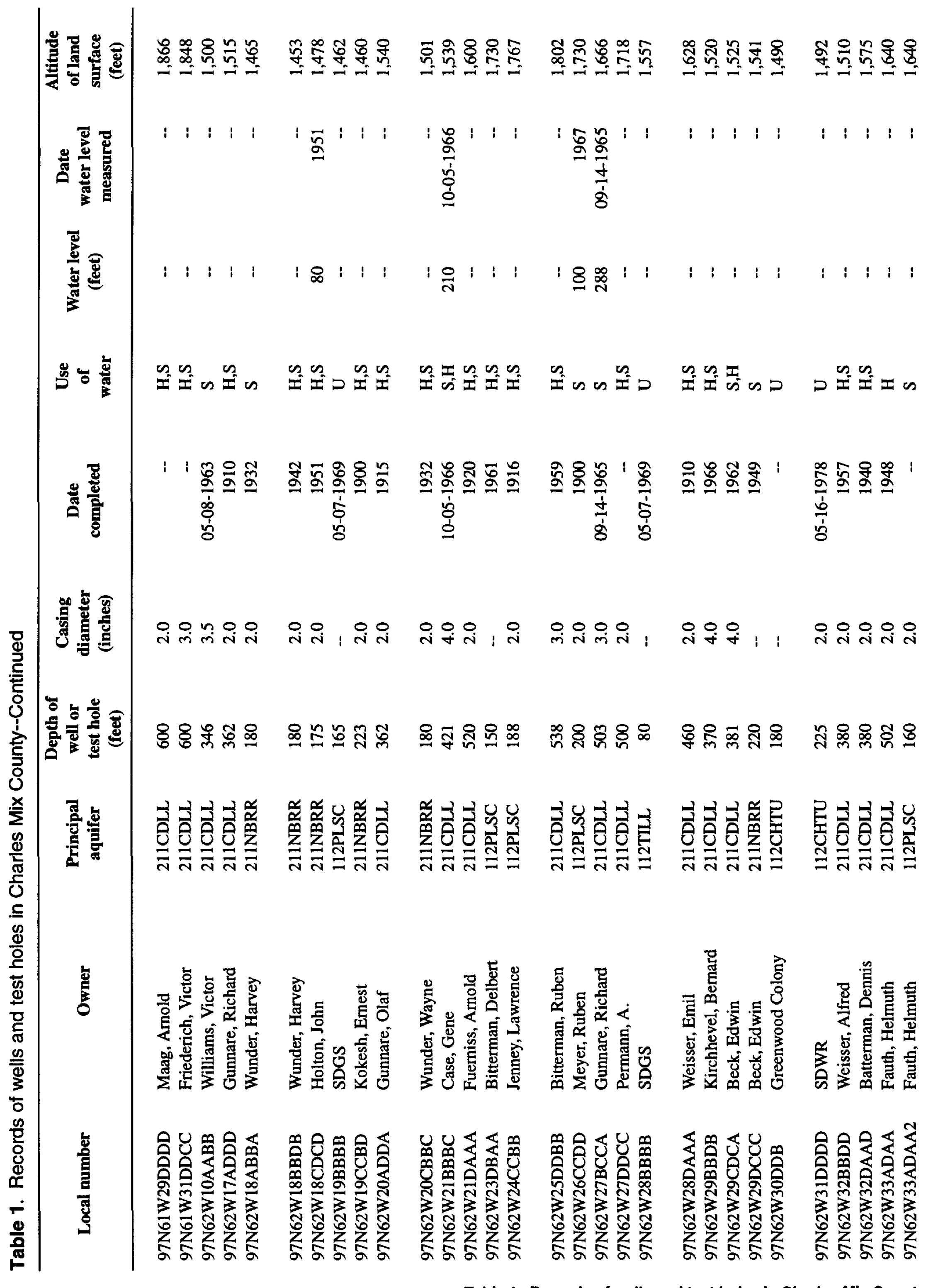




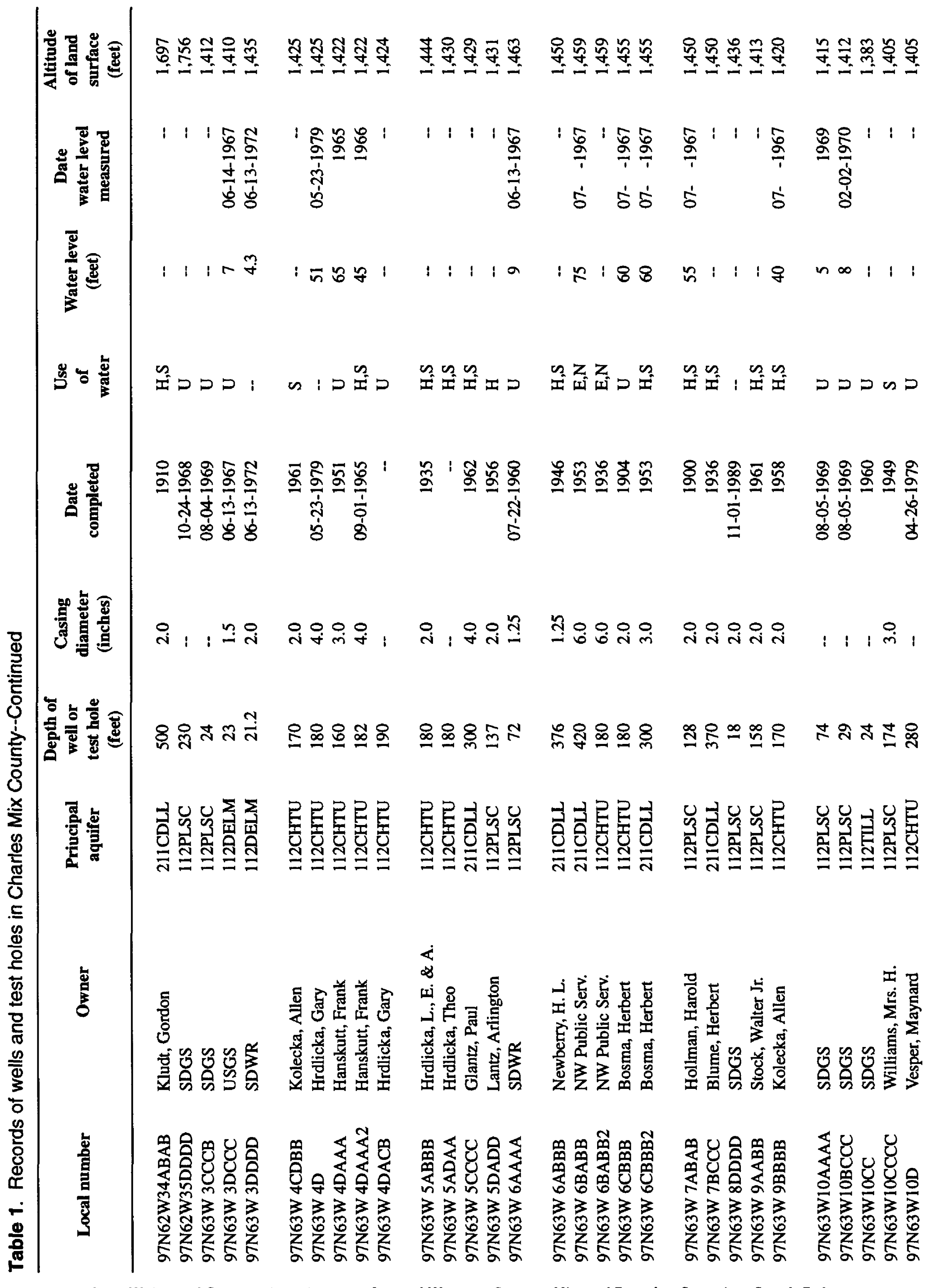

40 Records of Wells and Chemical Analyses of Ground Water in Charles Mix and Douglas Counties, South Dakota 


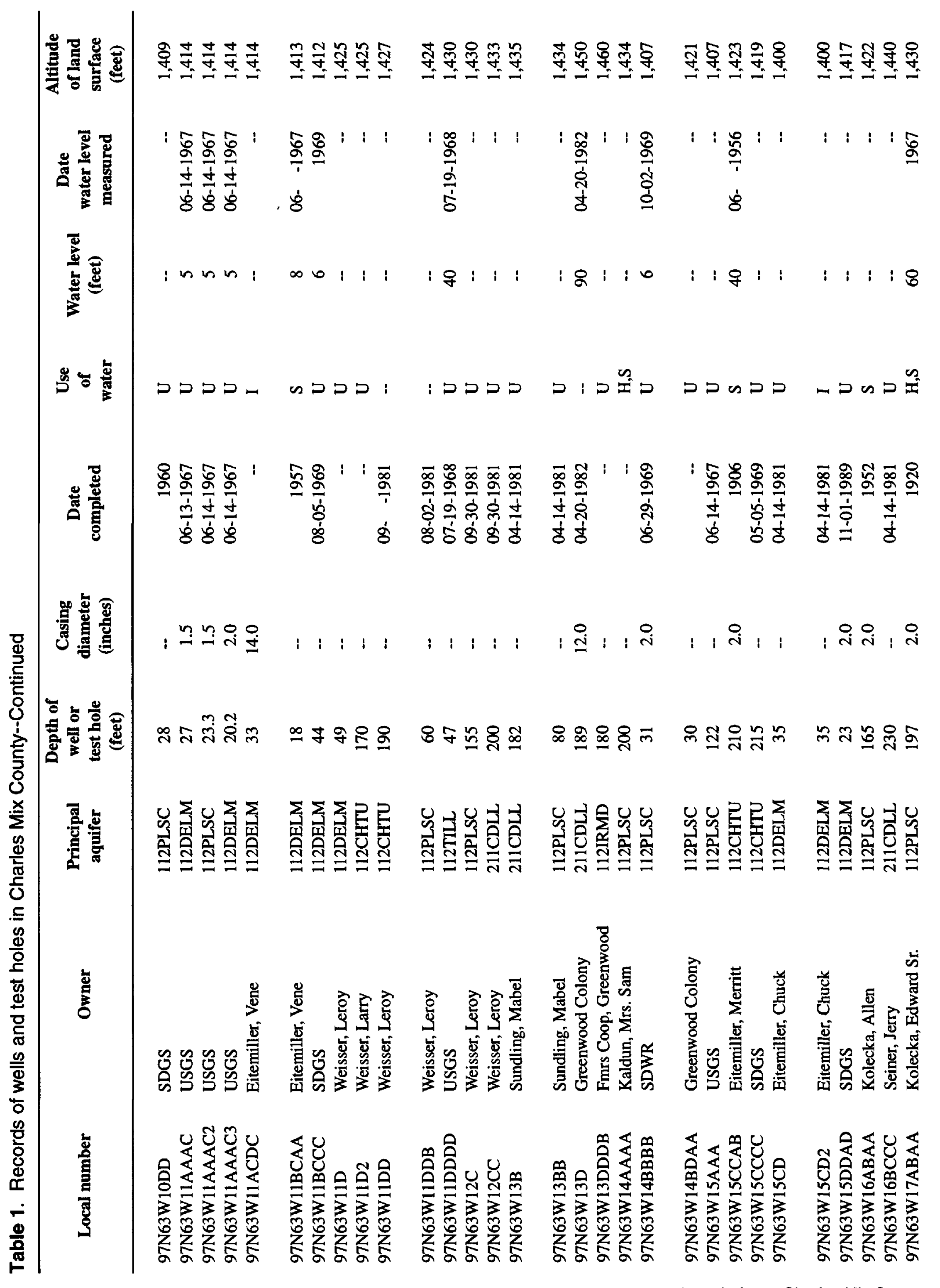

Table 1. Records of wells and test holes in Charles Mix County 41 


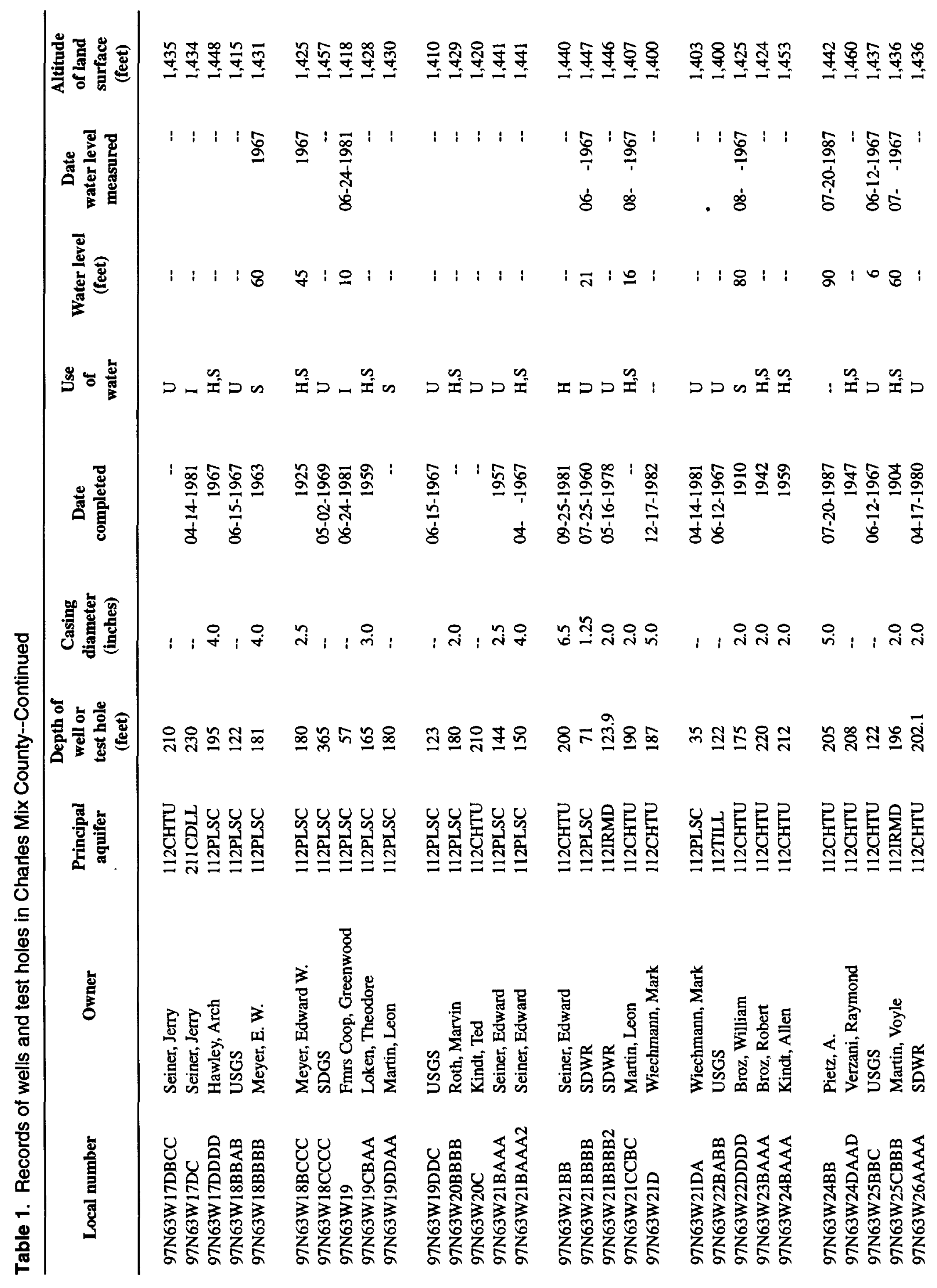

42 Records of Wells and Chemicel Analyses of Ground Water in Cherles Mix snd Douglas Counties, South Dakota 


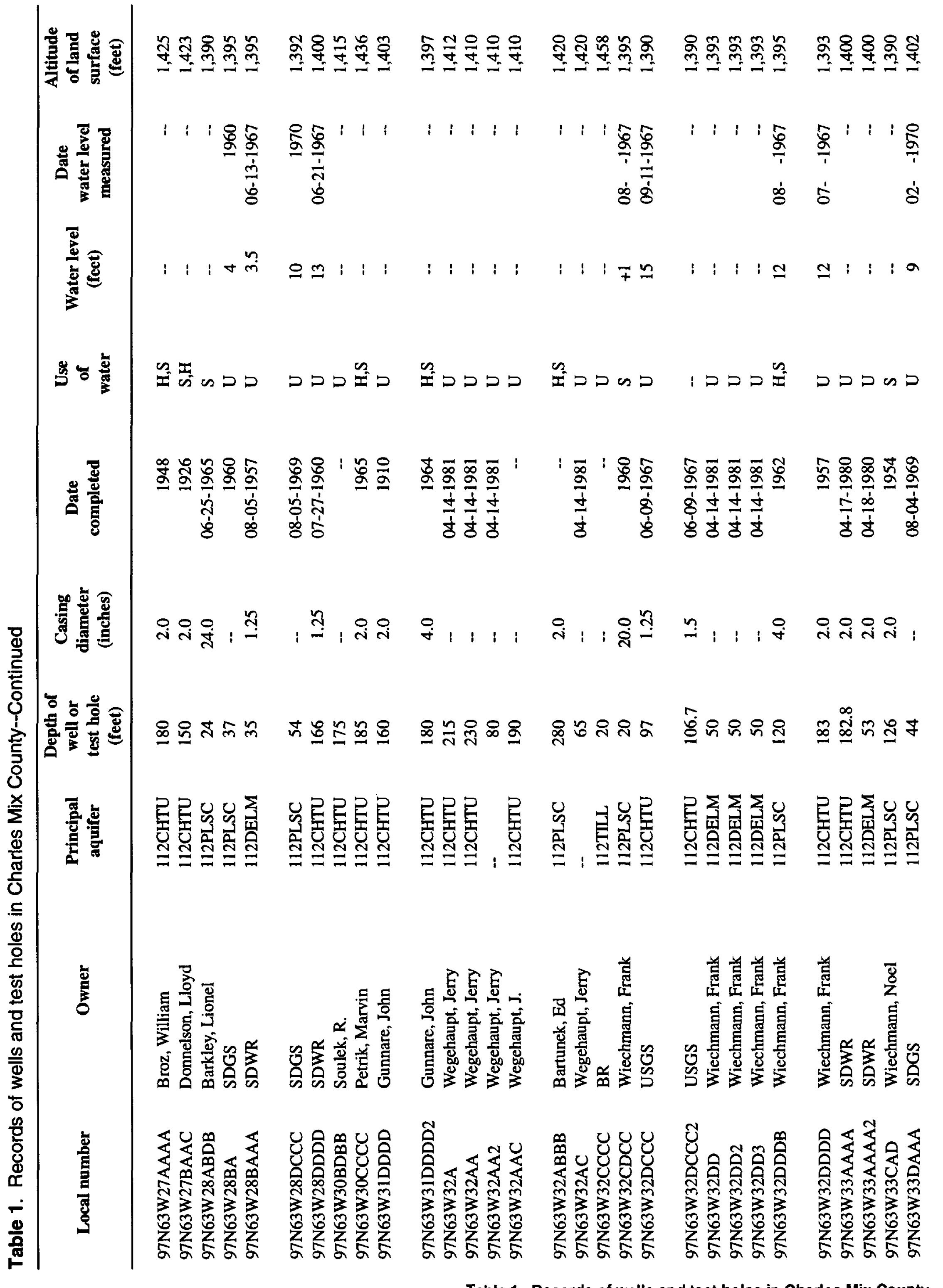




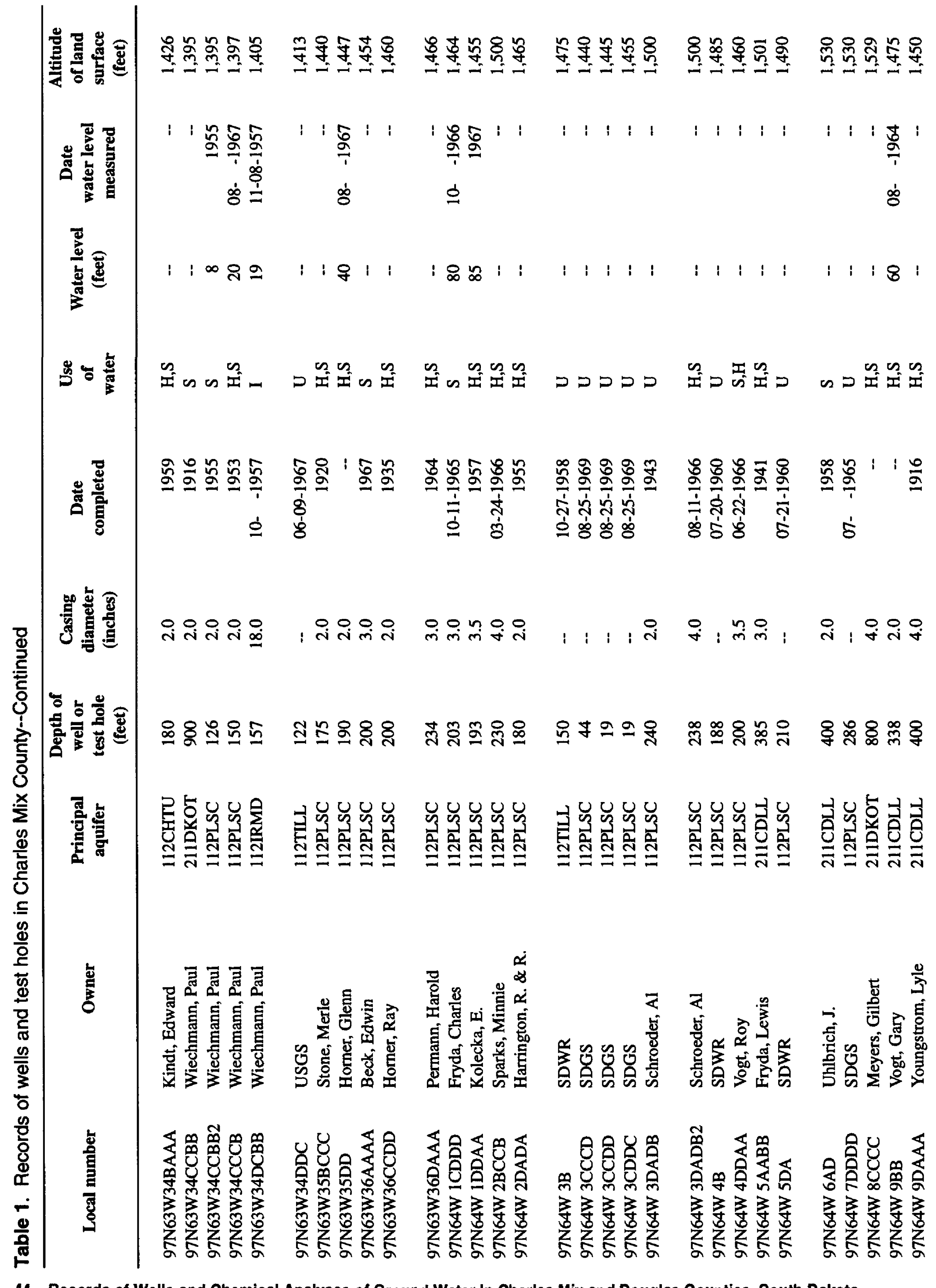

44 Records of Wells and Chemical Analyses of Ground Water In Charles Mix and Douglas Counties, South Dakota 


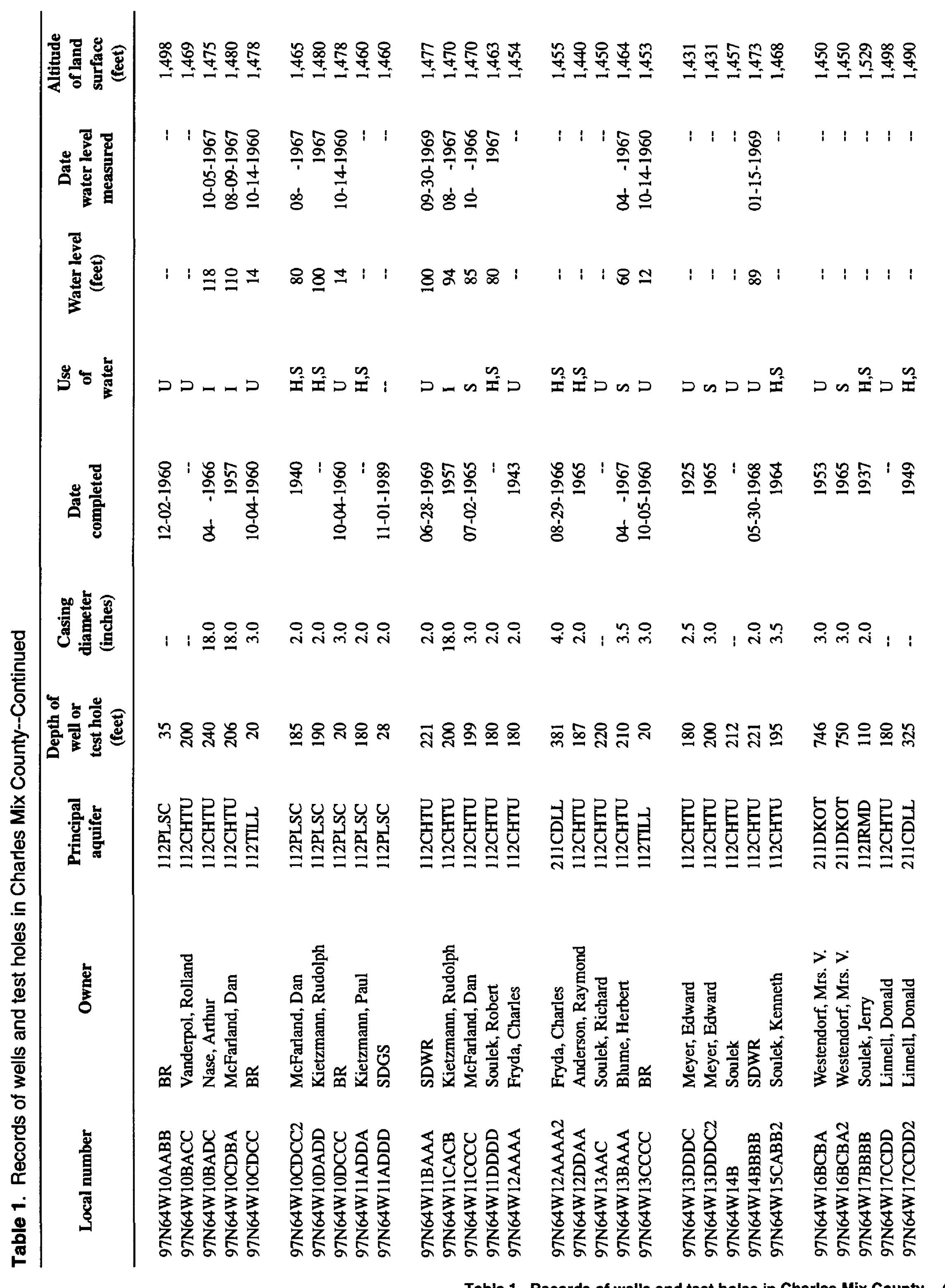




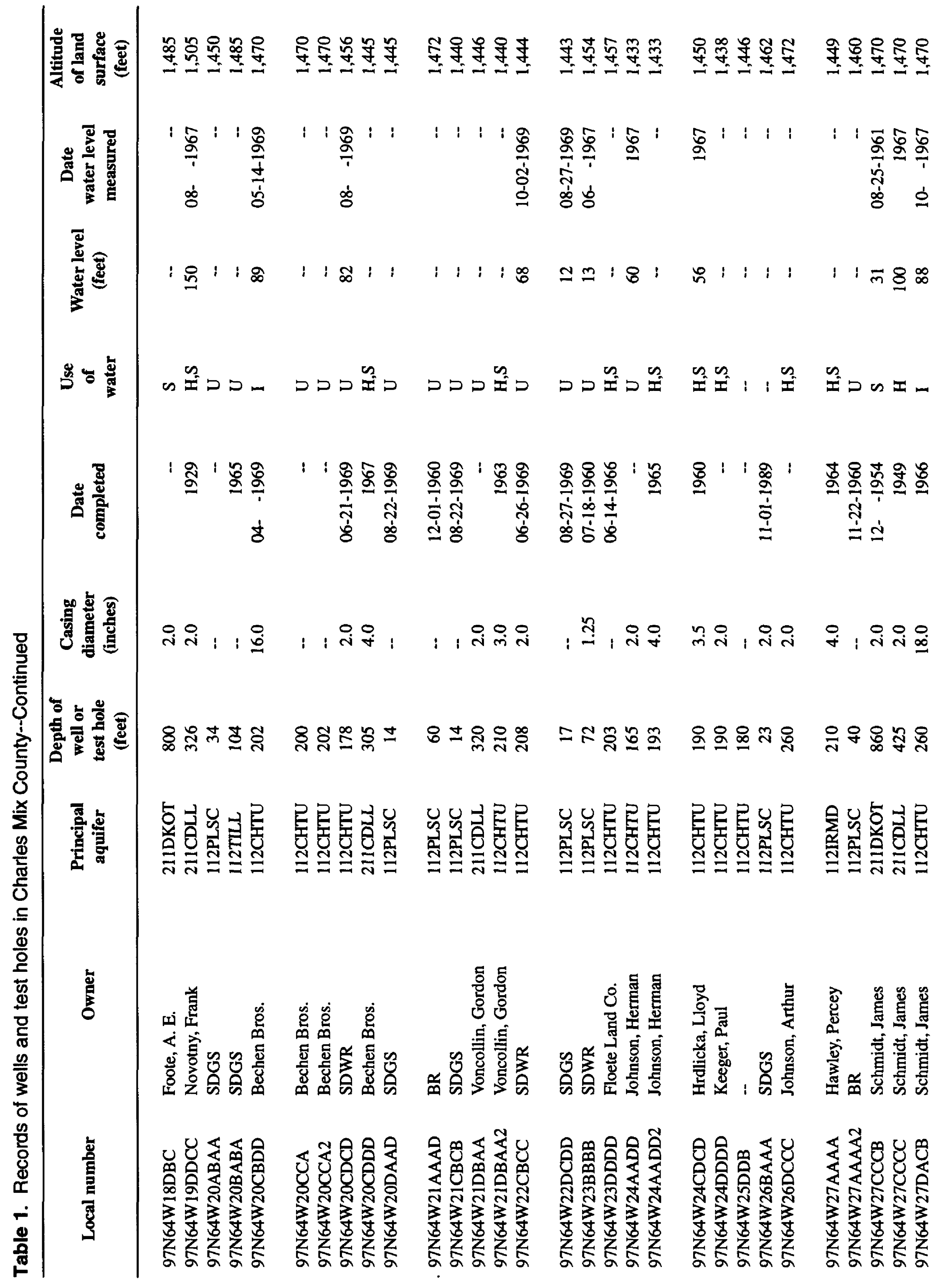

46 Records of Wells and Chemical Analyses of Ground Water in Chsrles Mix end Douglas Counties, South Dakota 


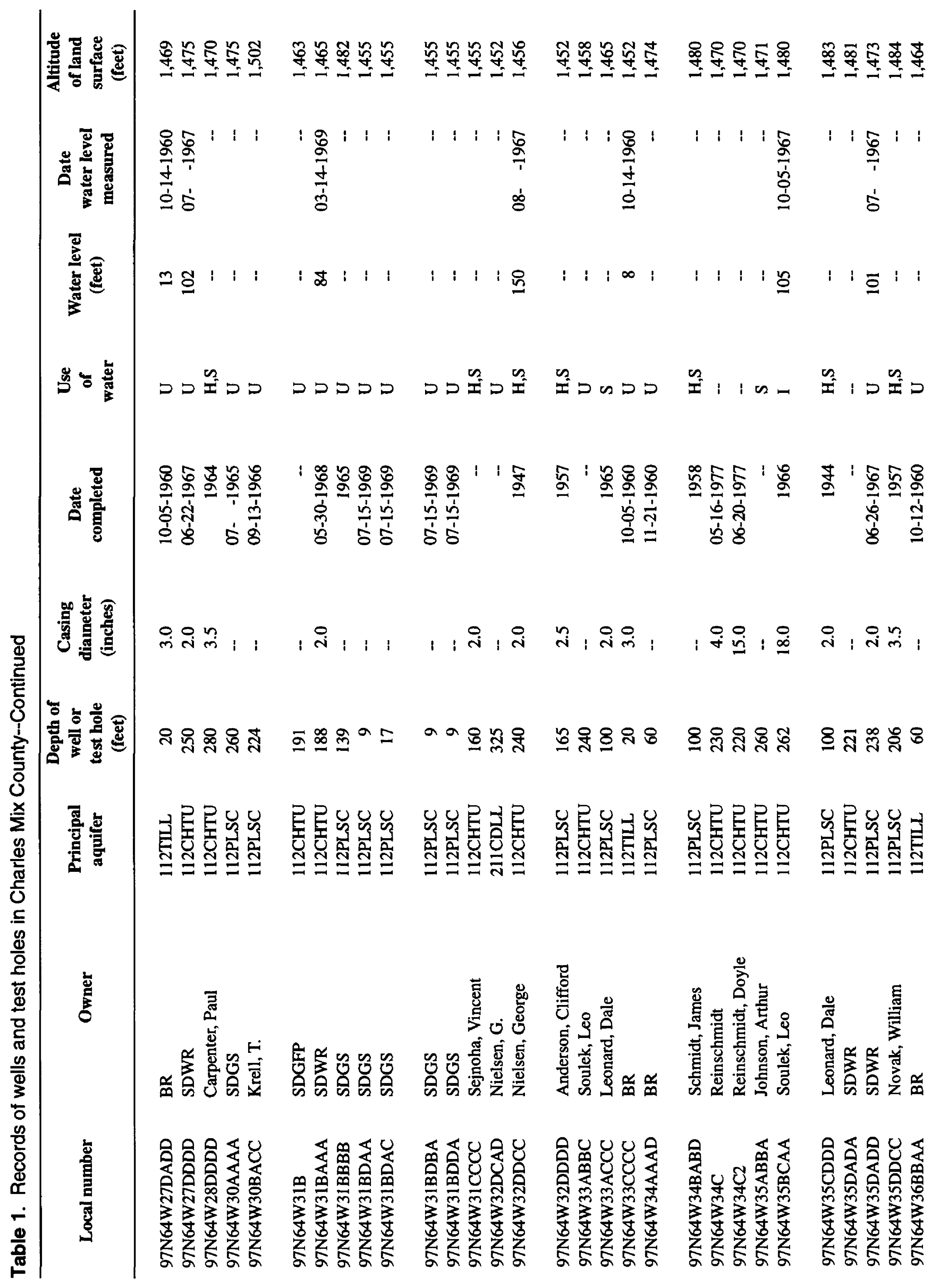

Table 1. Records of wells and test holes in Charles Mix County 47 


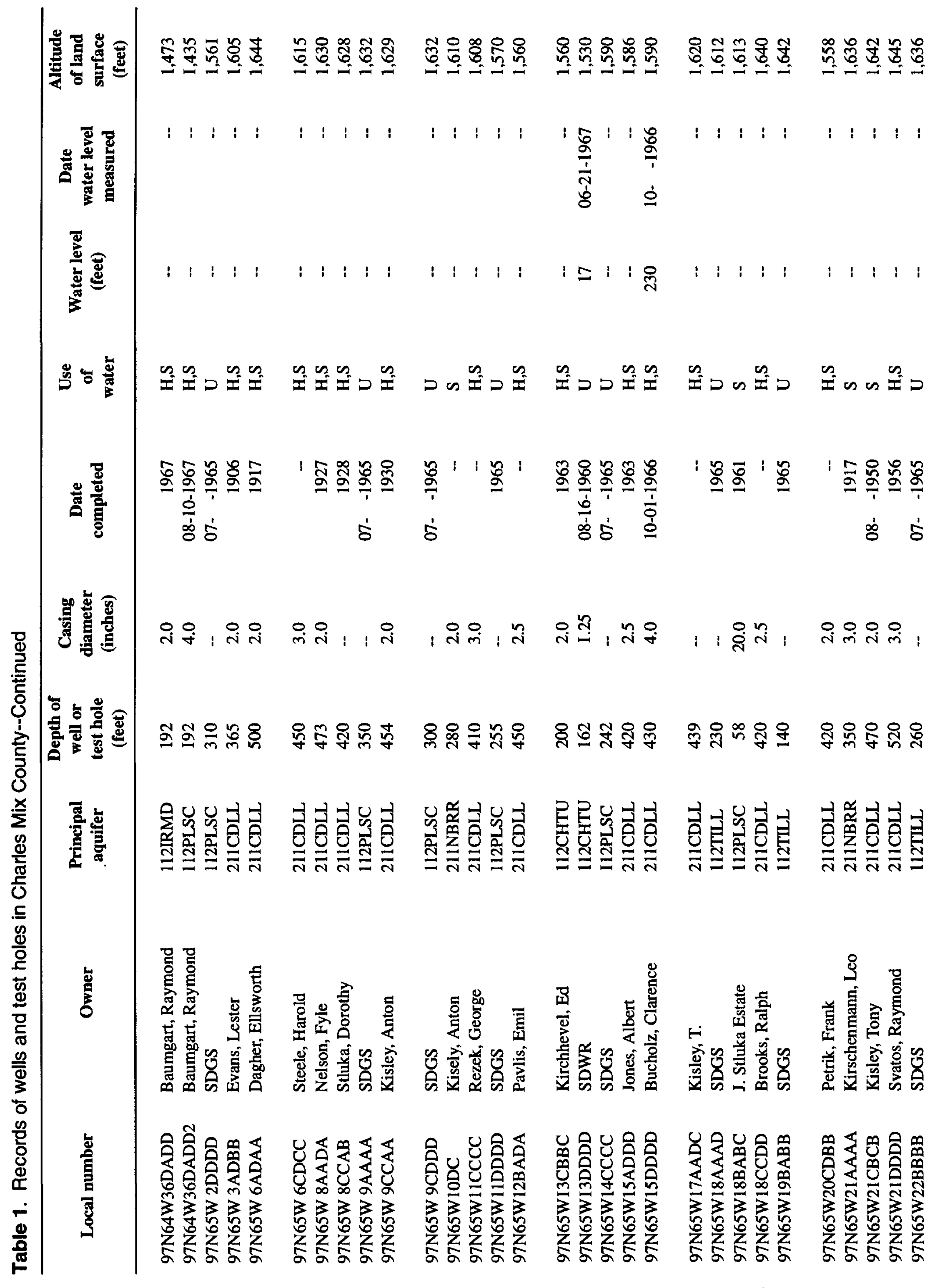

48 Records of Wells and ChemicsI Analyses of Ground Water in Chsrles Mix snd Douglas Counties, South Dakota 


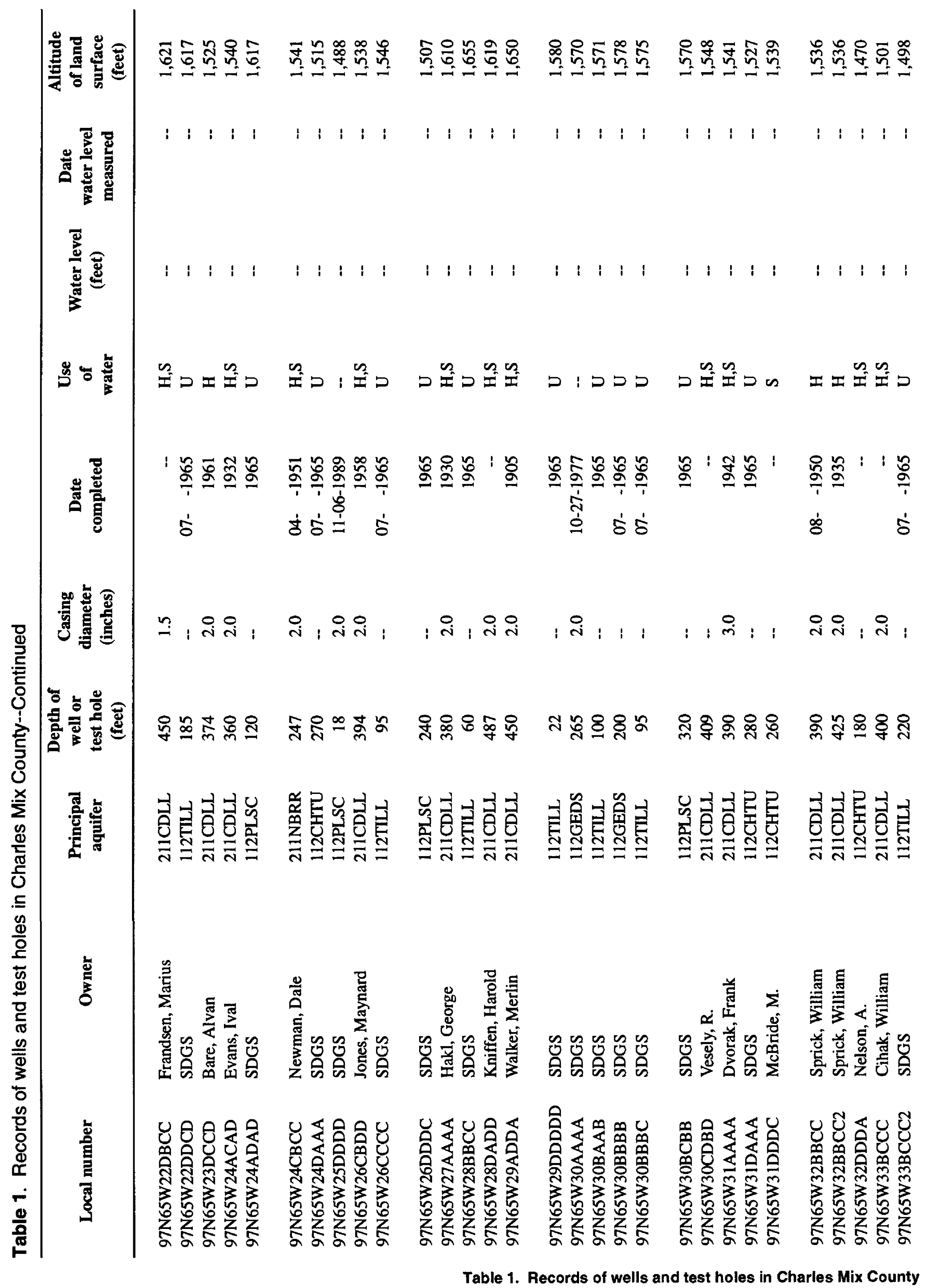




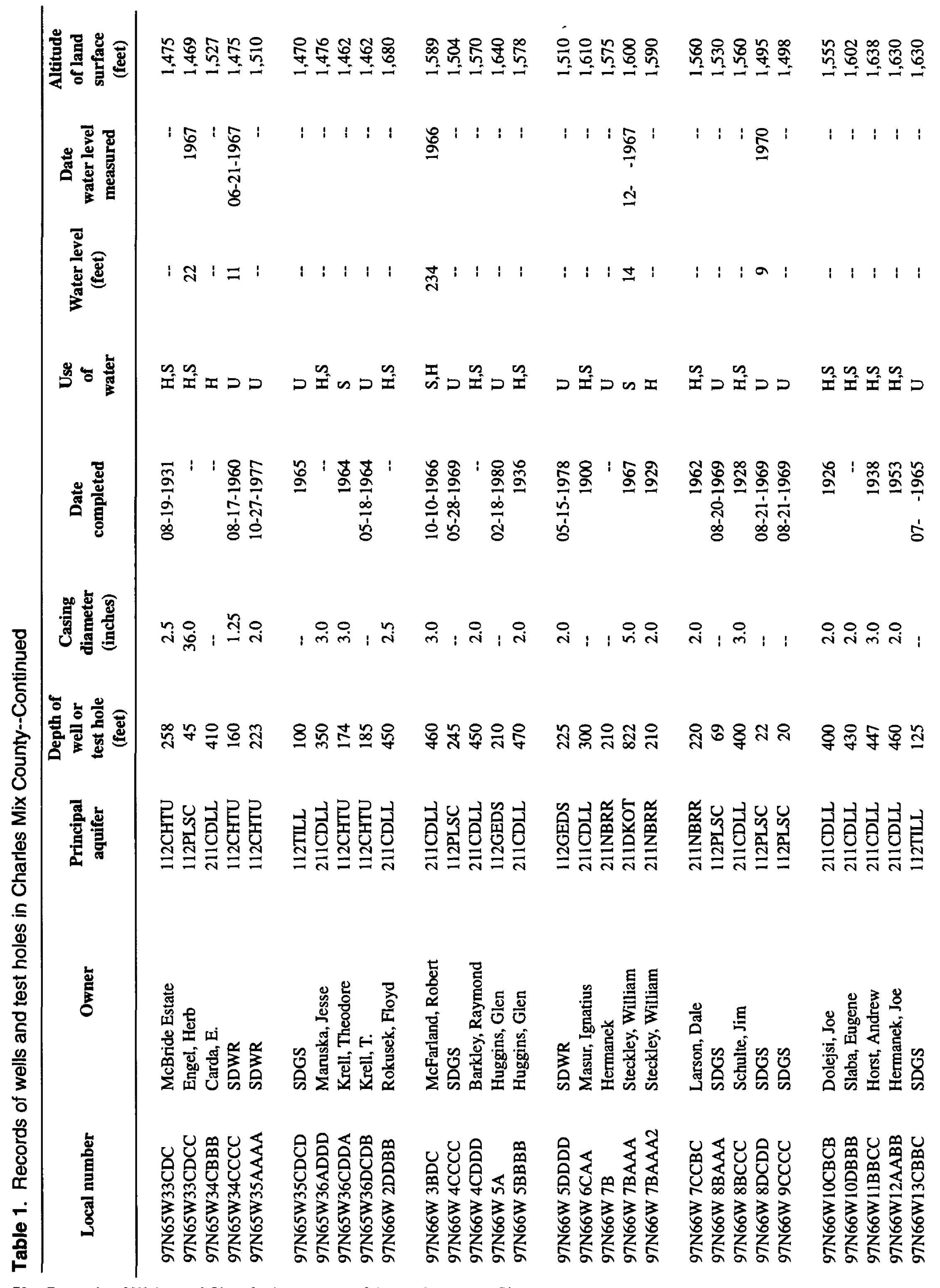

50 Records of Wells and Chemical Analyaas of Ground Water in Charlaa Mix and Douglas Counties, South Dakota 


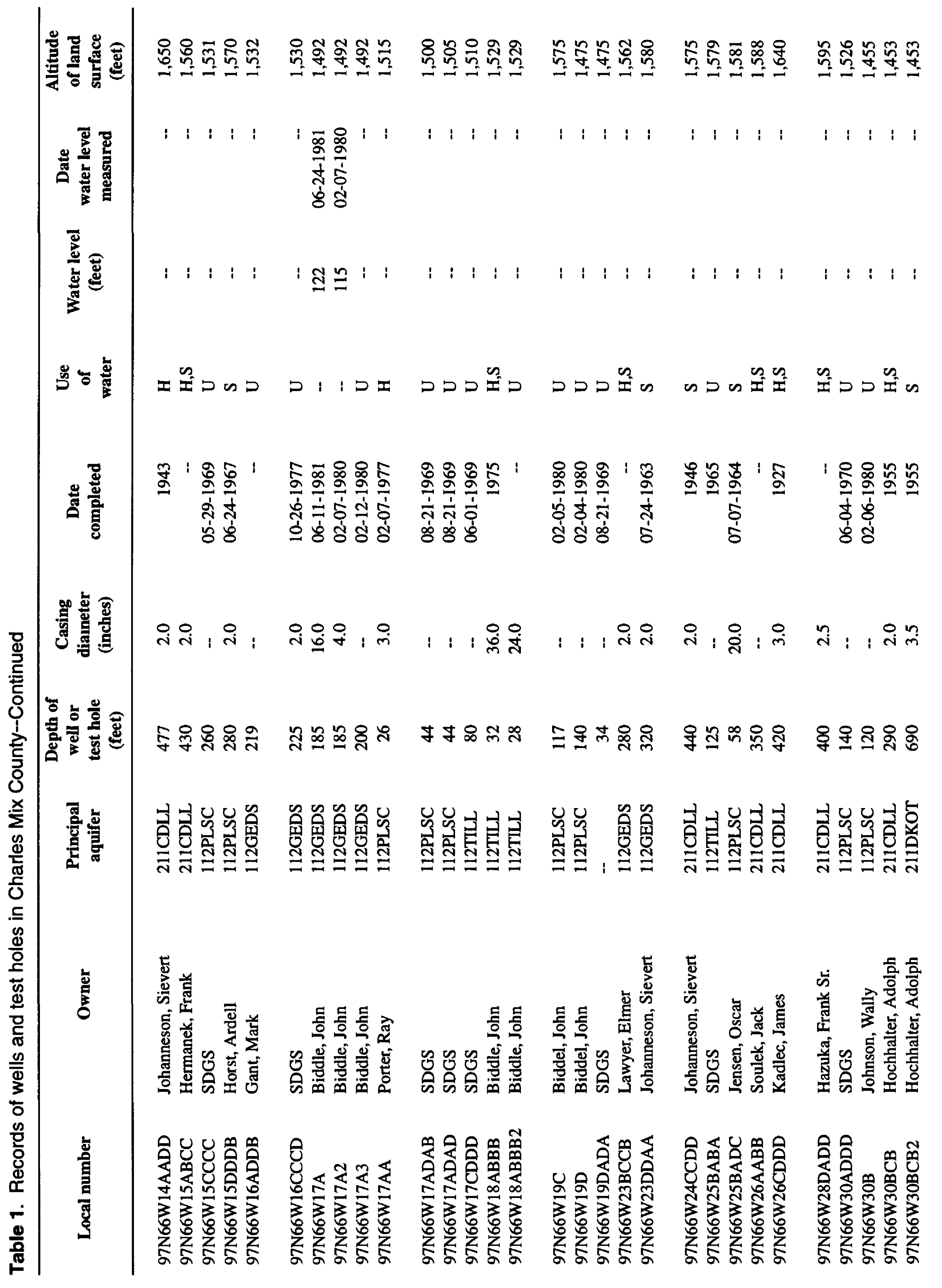




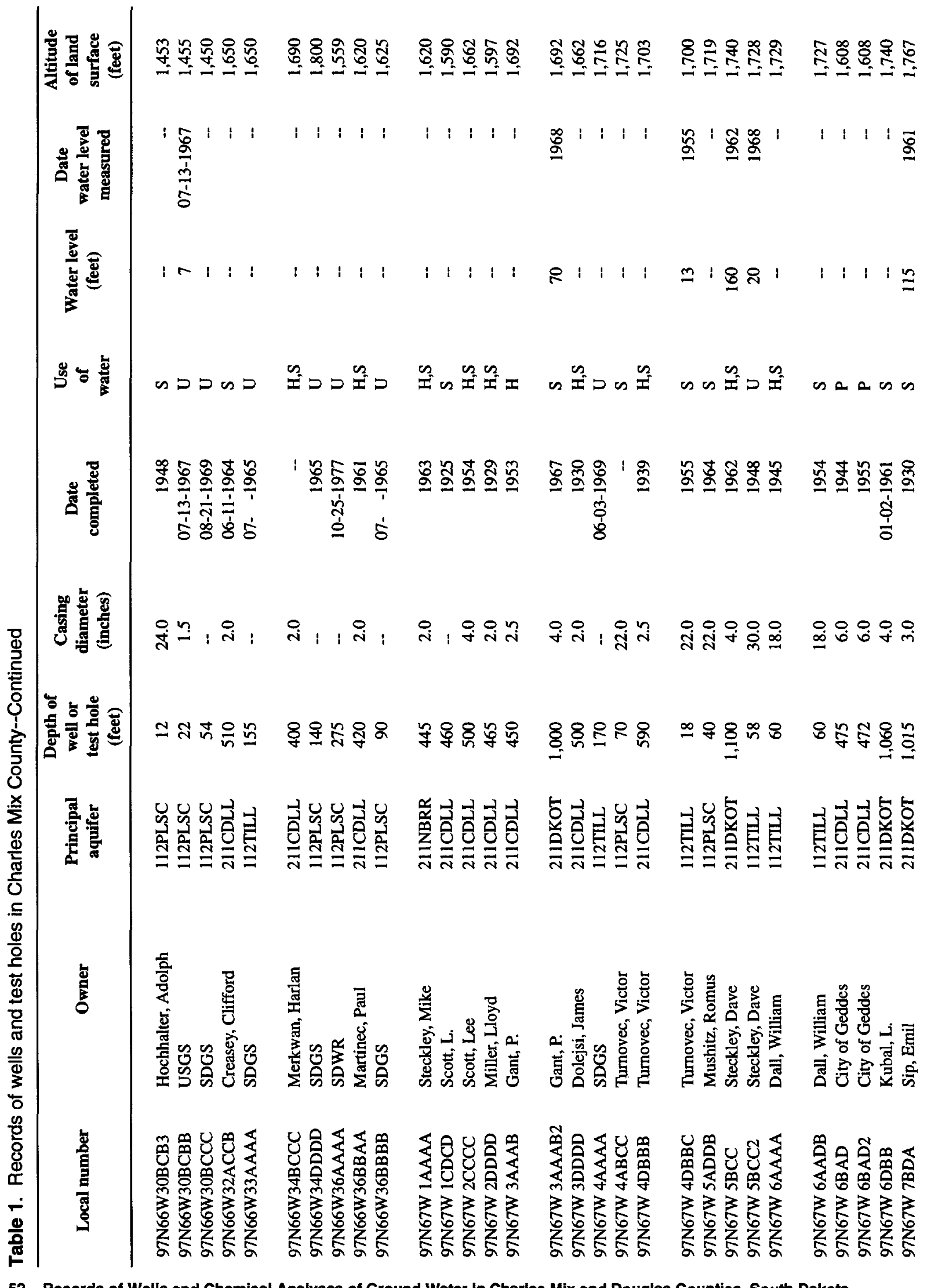

52 Records of Wells and Chemical Analyses of Ground Water In Charles Mix and Douglas Counties, South Dakota 


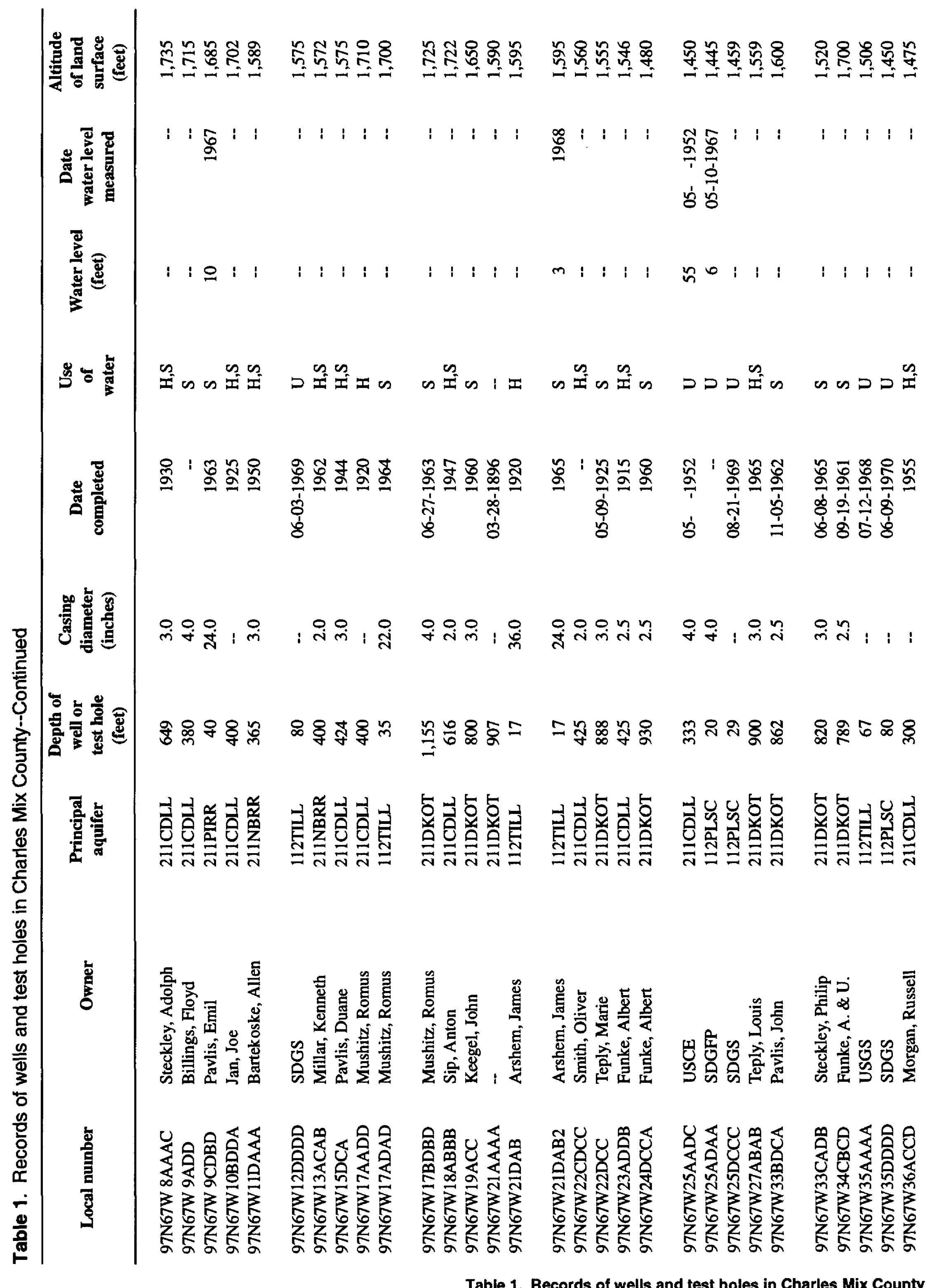




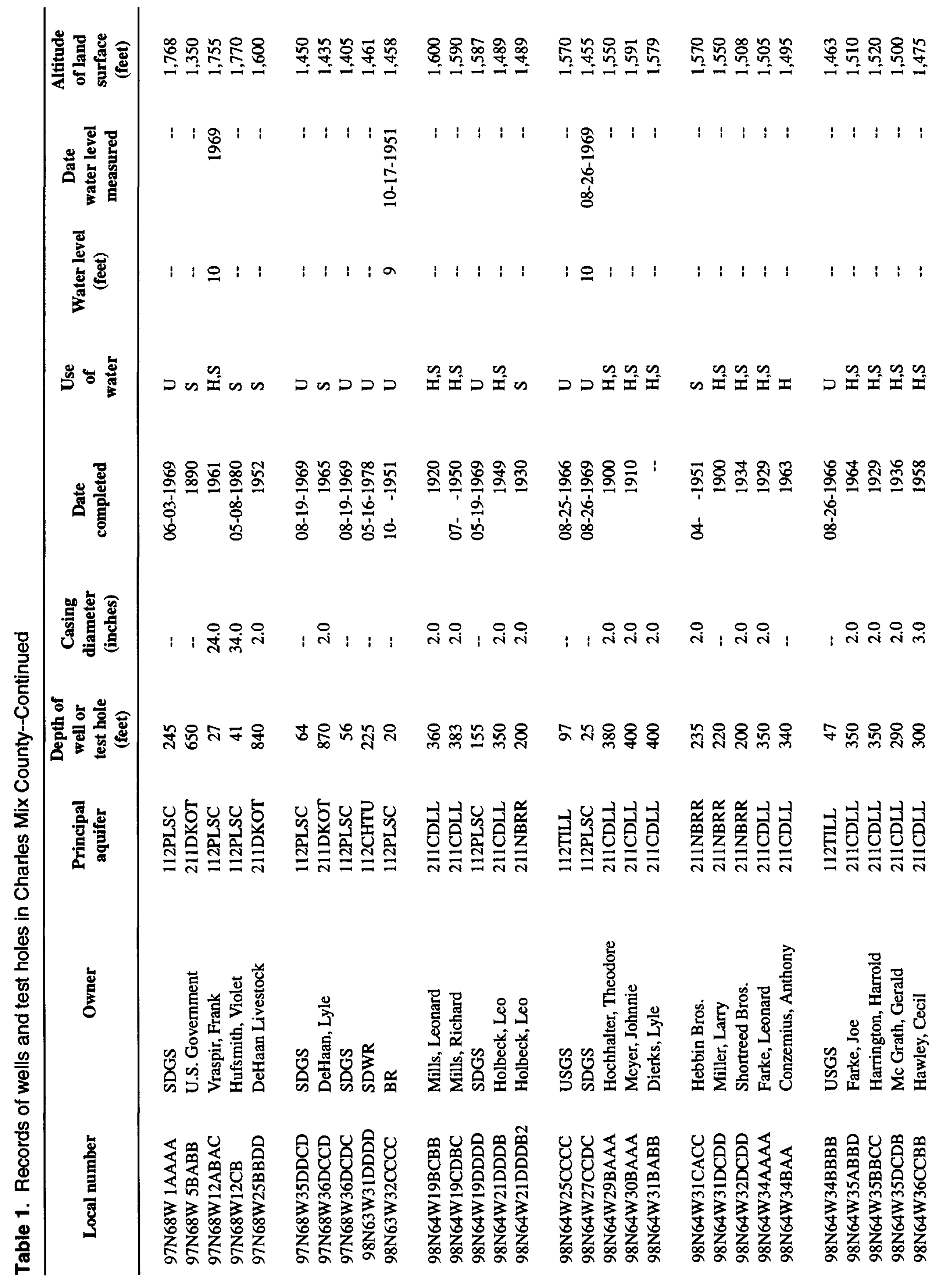




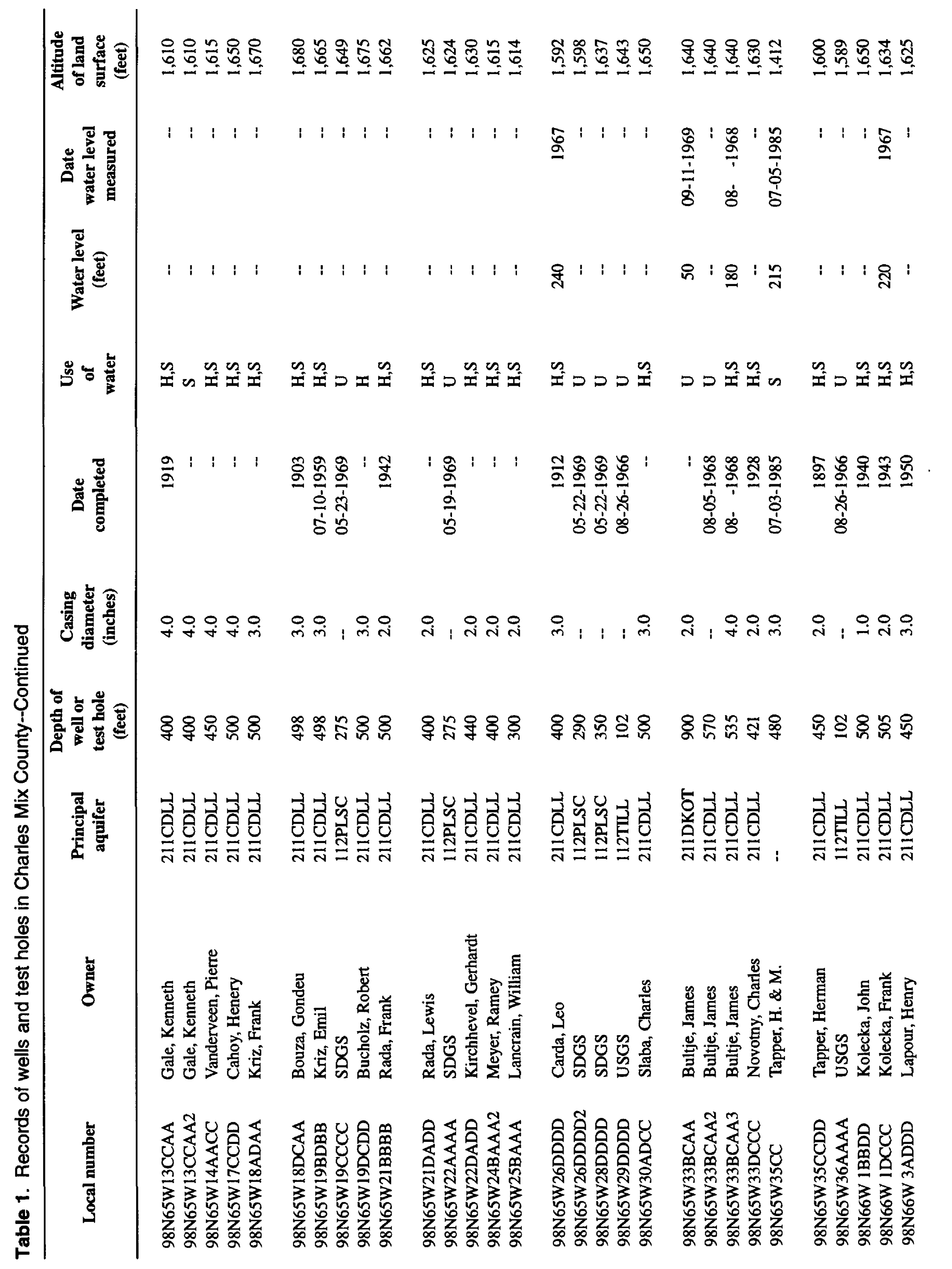




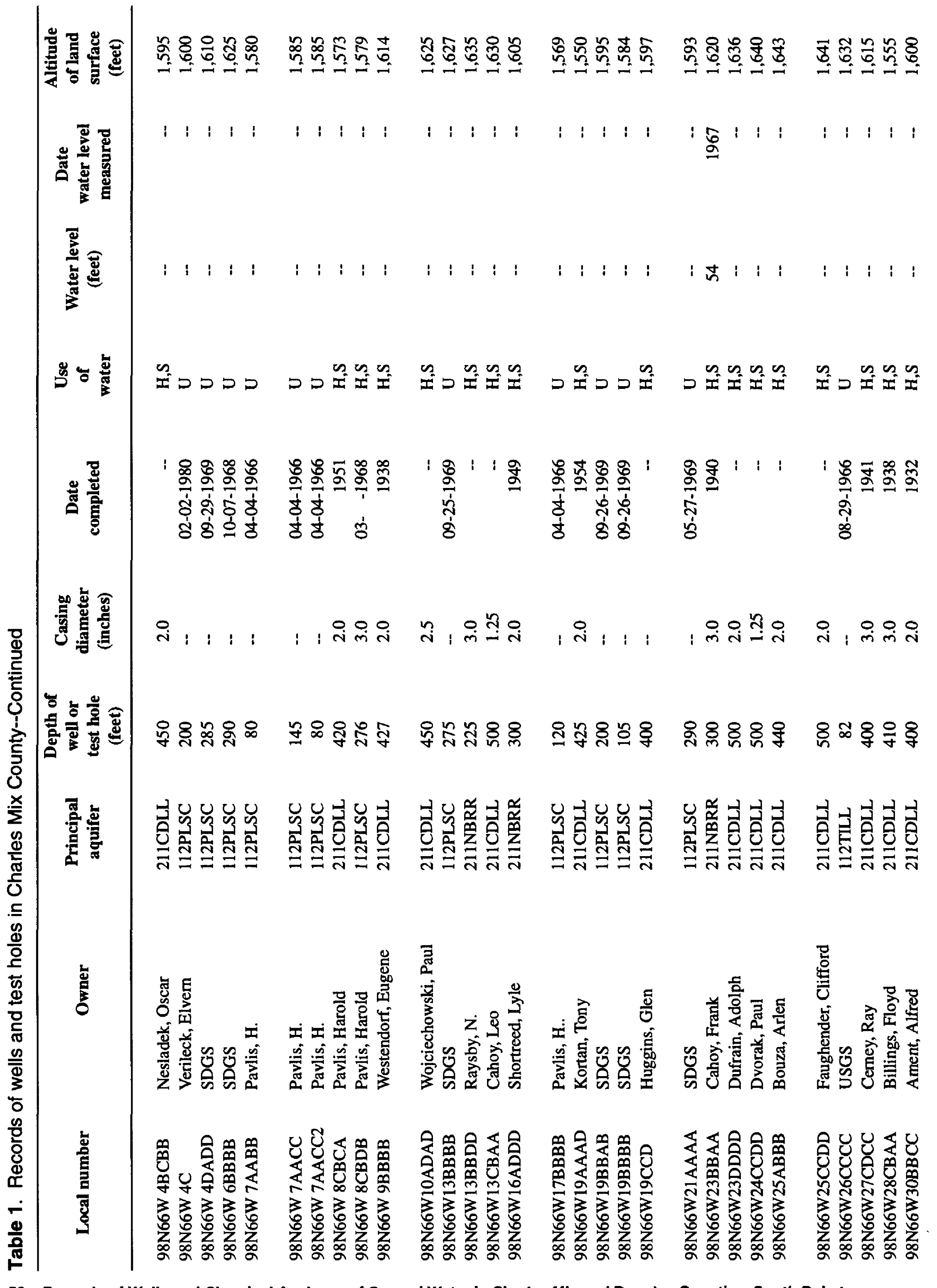

56 Records of Wells and Chemical Analyses of Ground Water in Cherles Mix snd Douglas Counties, South Dakota 


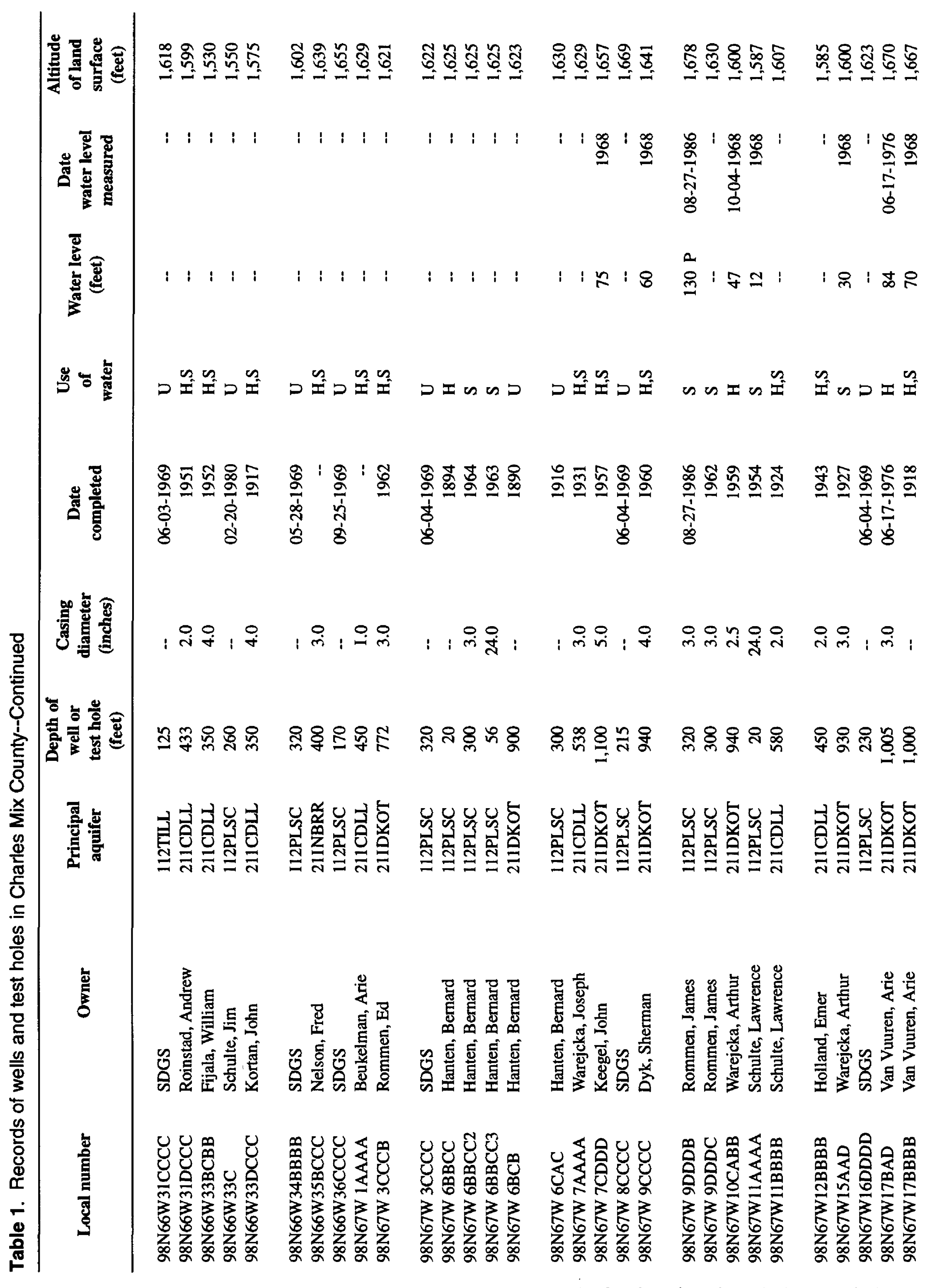

Table 1. Records of wells and test holes in Charies Mix County 


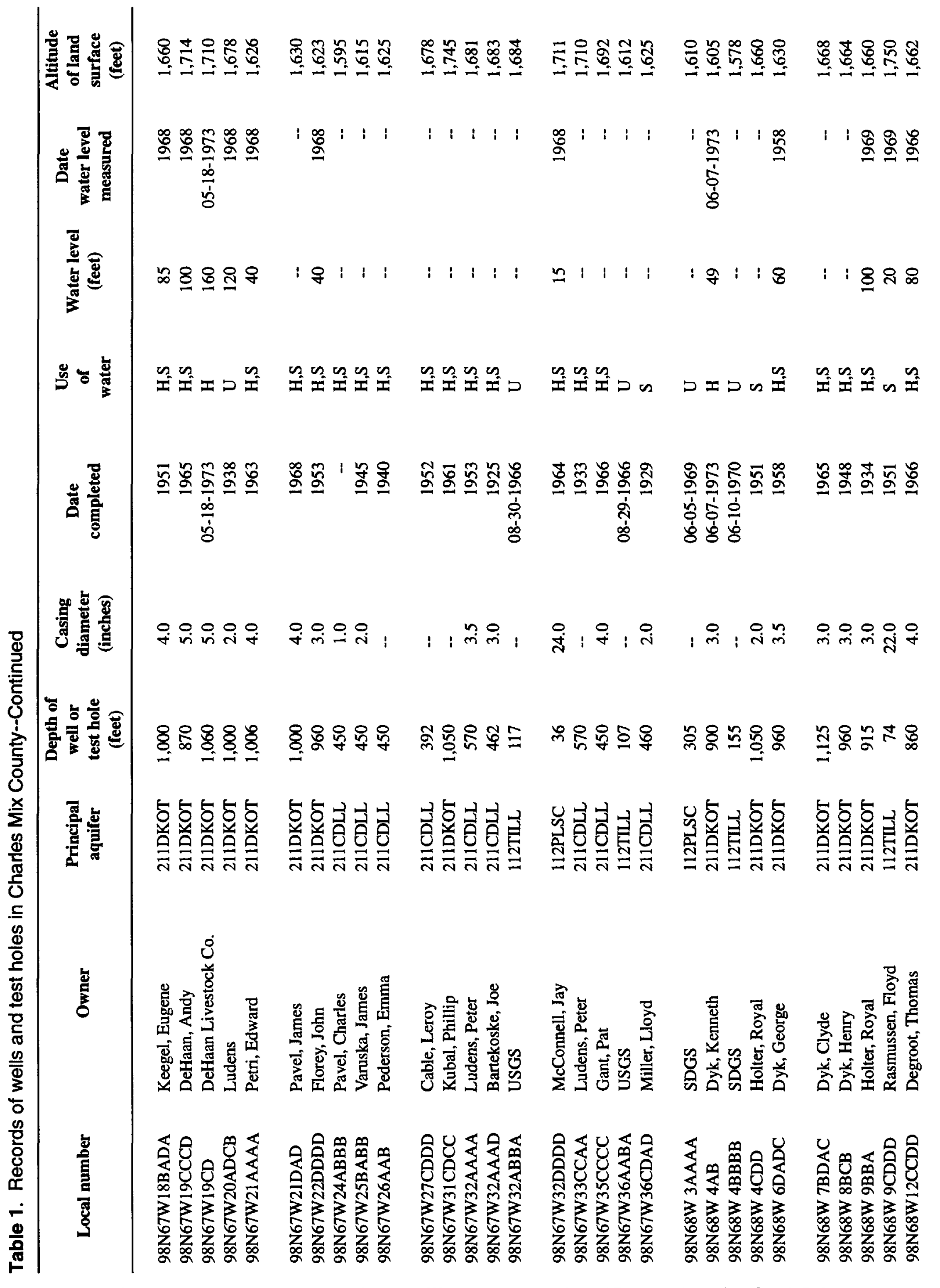

58 Records of Wells and Chemical Analyses of Ground Water in Charles Mix and Douglas Counties, South Dakota 


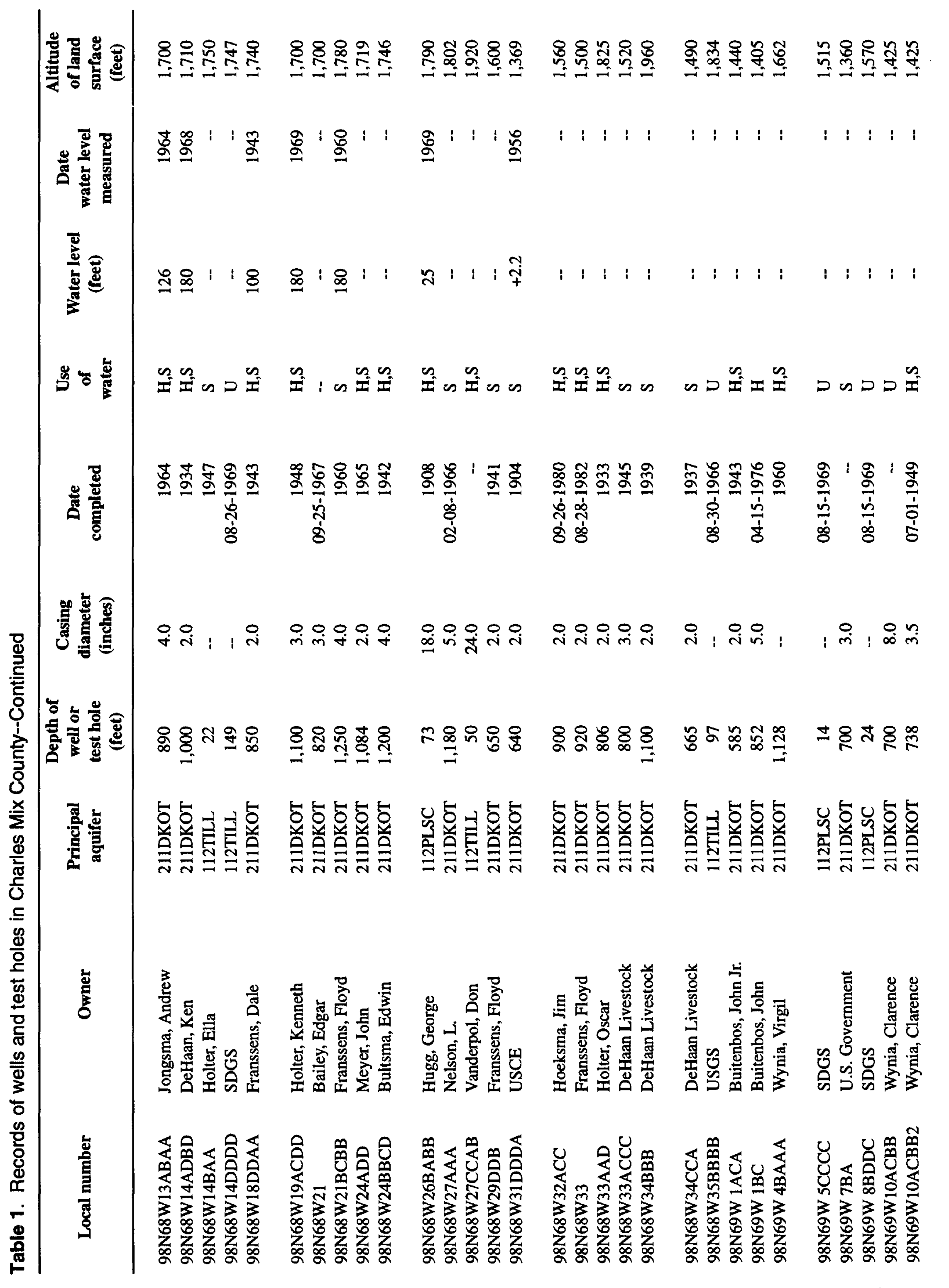




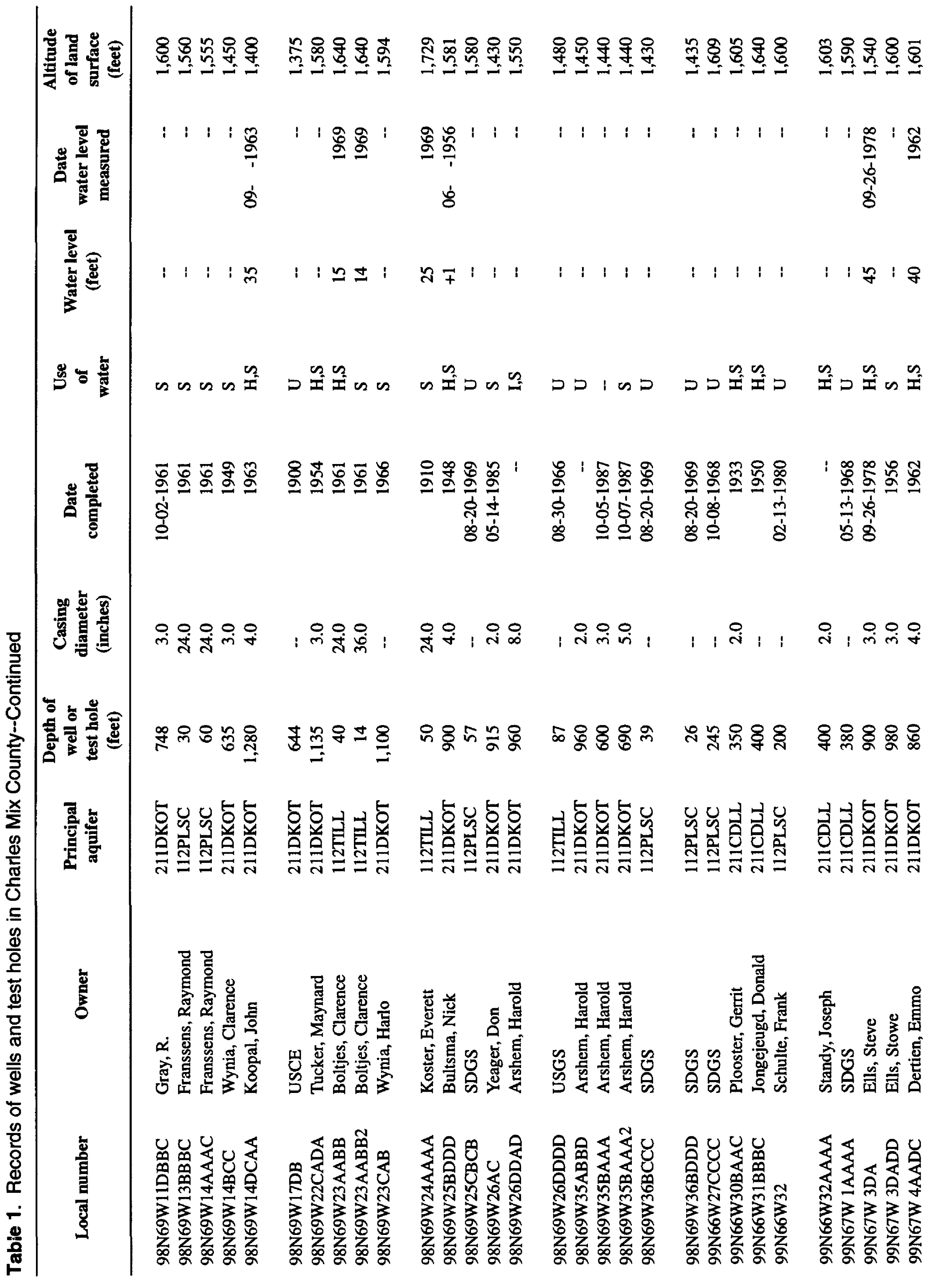

60 Records of Weils and Chemical Analyses of Ground Water In Chsrles Mix and Douglas Counties, South Dakota 


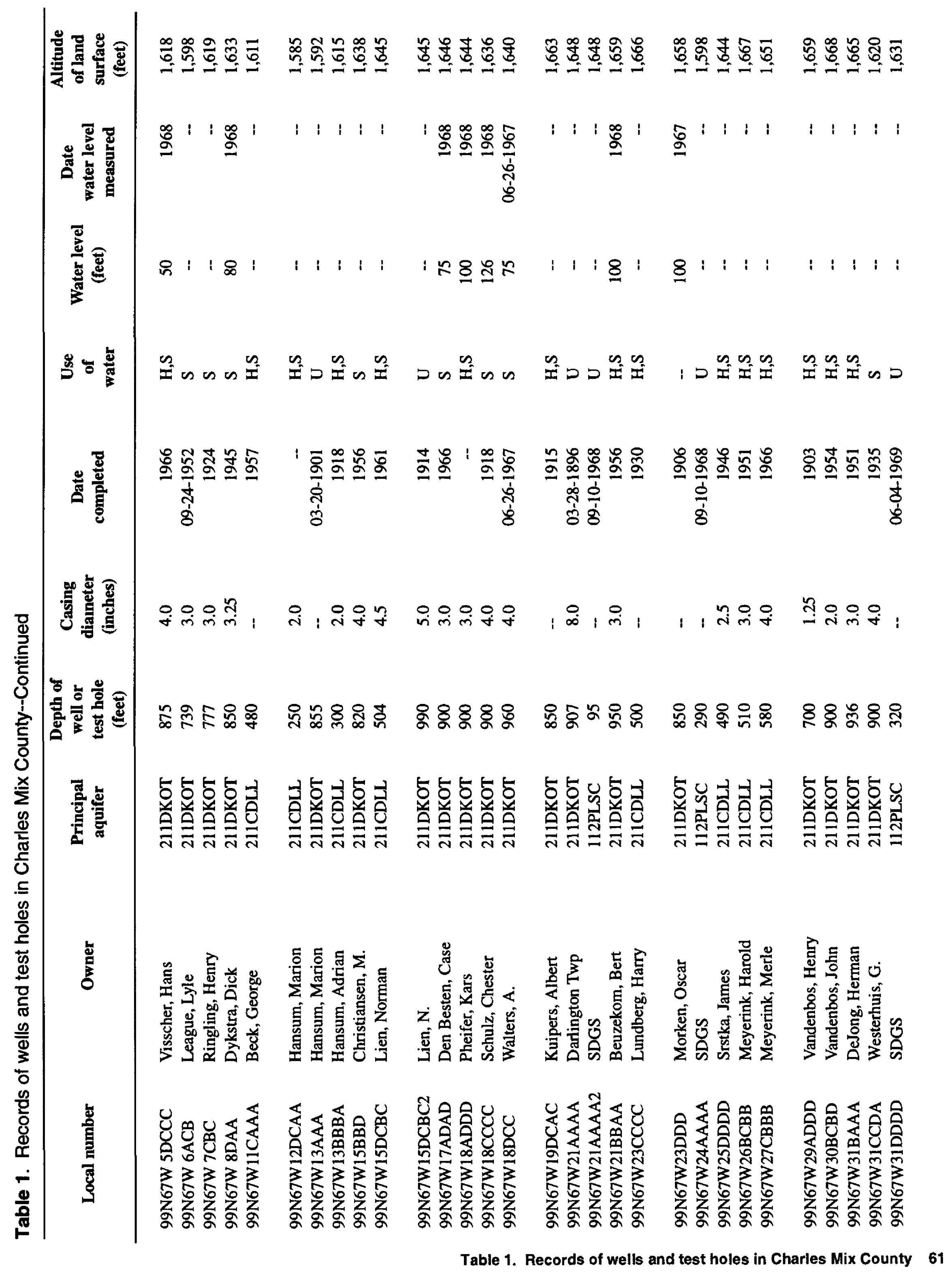




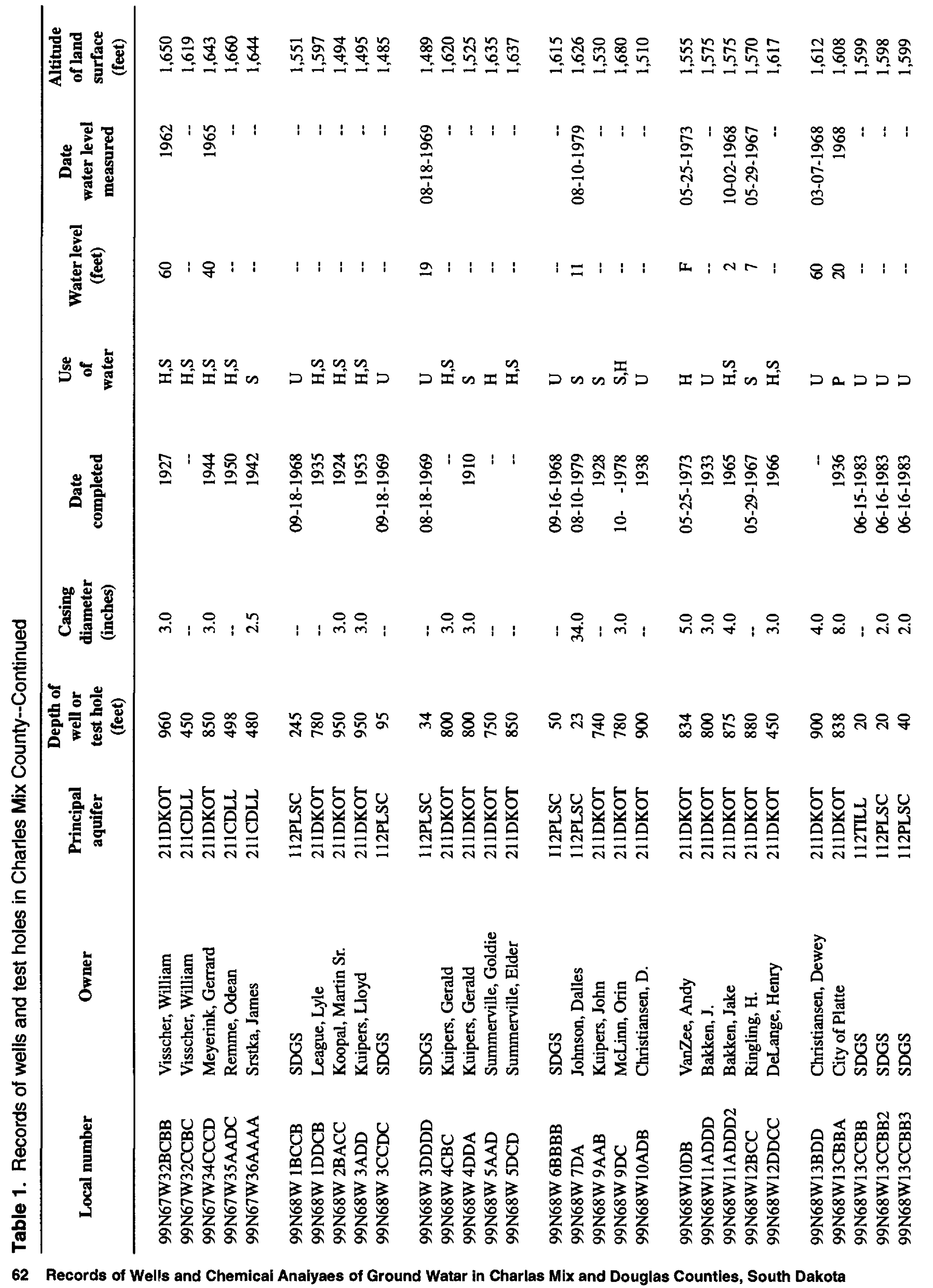




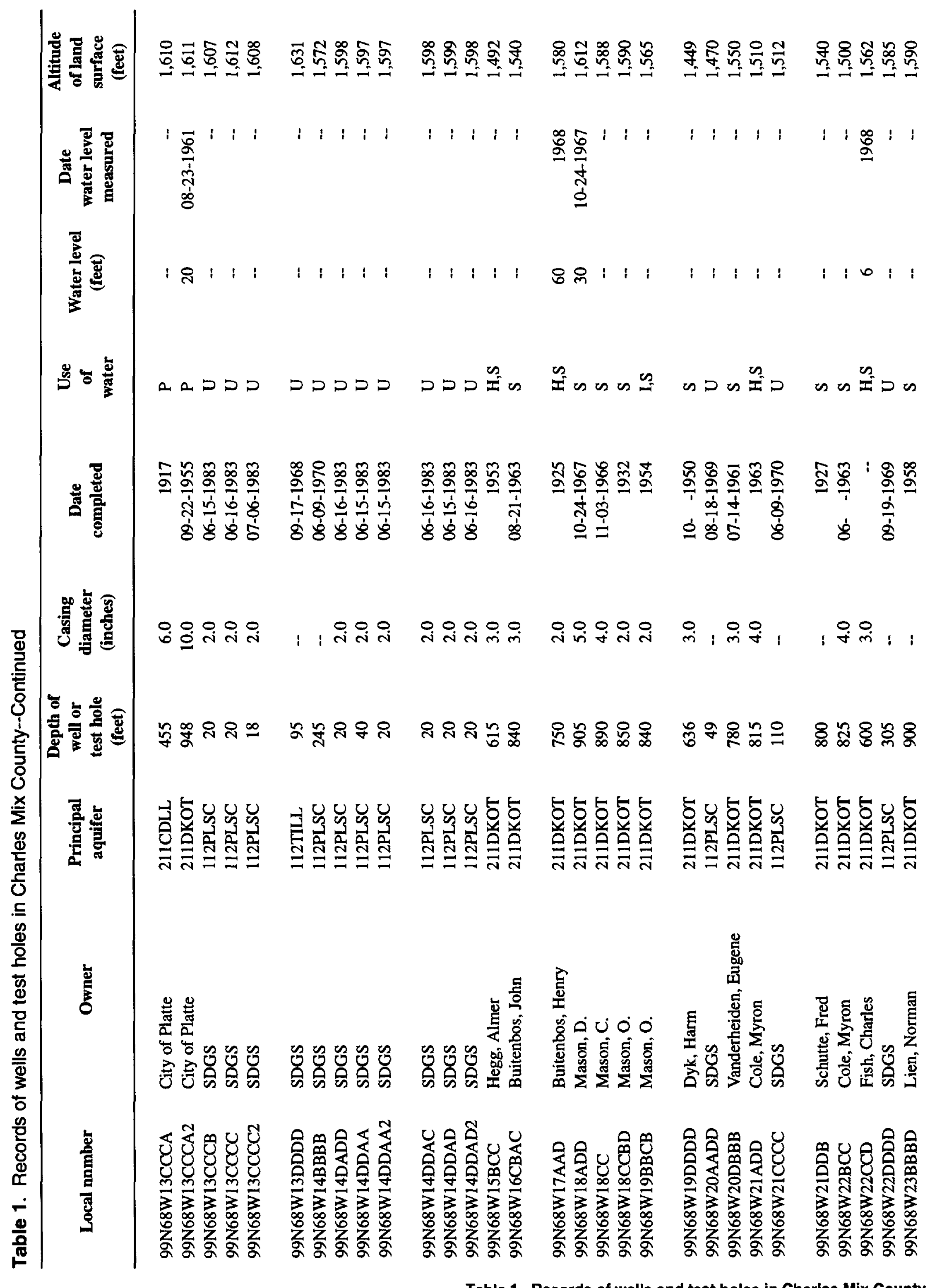

Table 1. Records of wells and test holes in Charles Mix County 63 


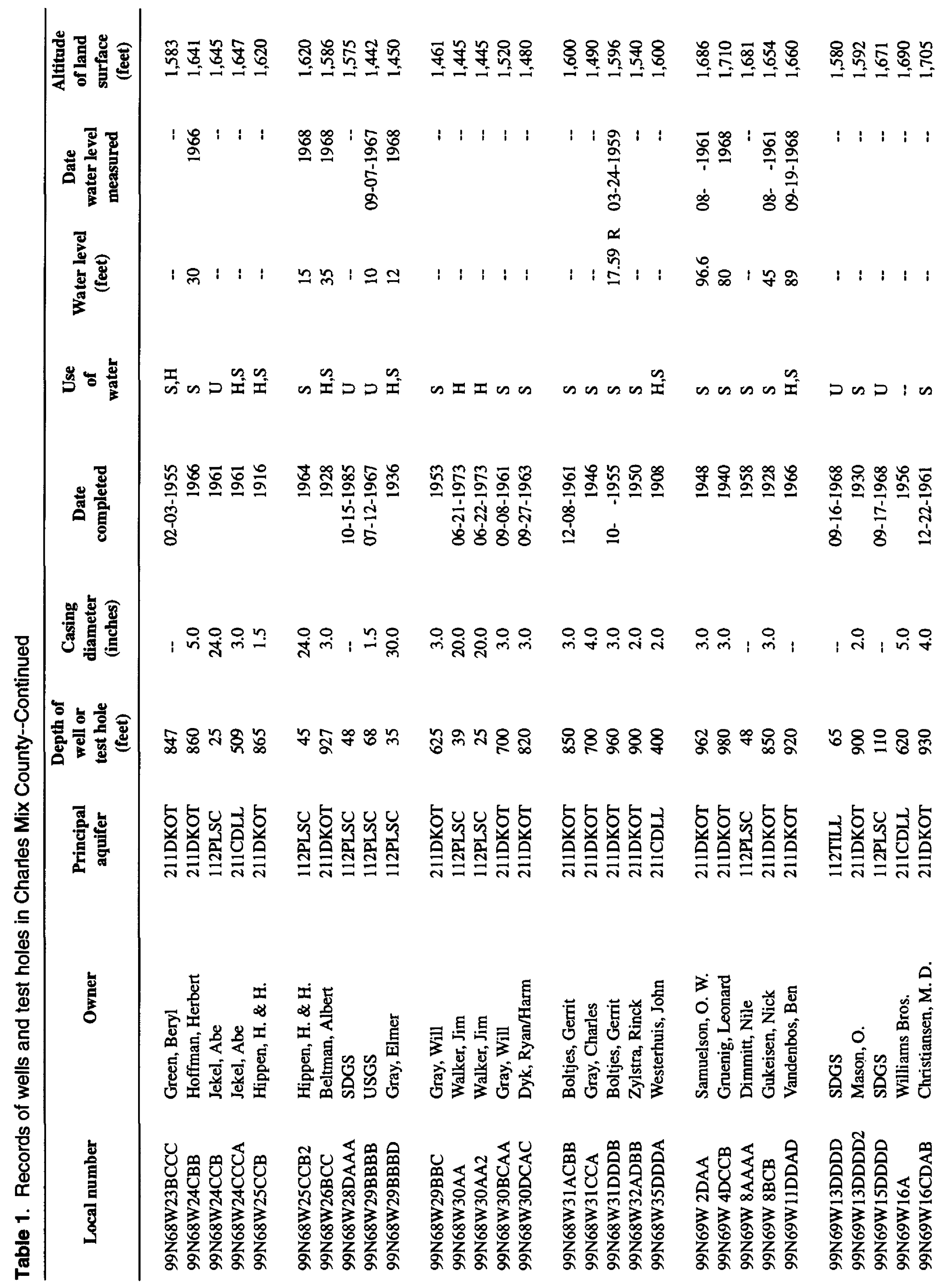

64 Records of Wells and Chemicsl Analyses of Ground Water in Chsrles Mix and Douglas Counties, South Dakota 


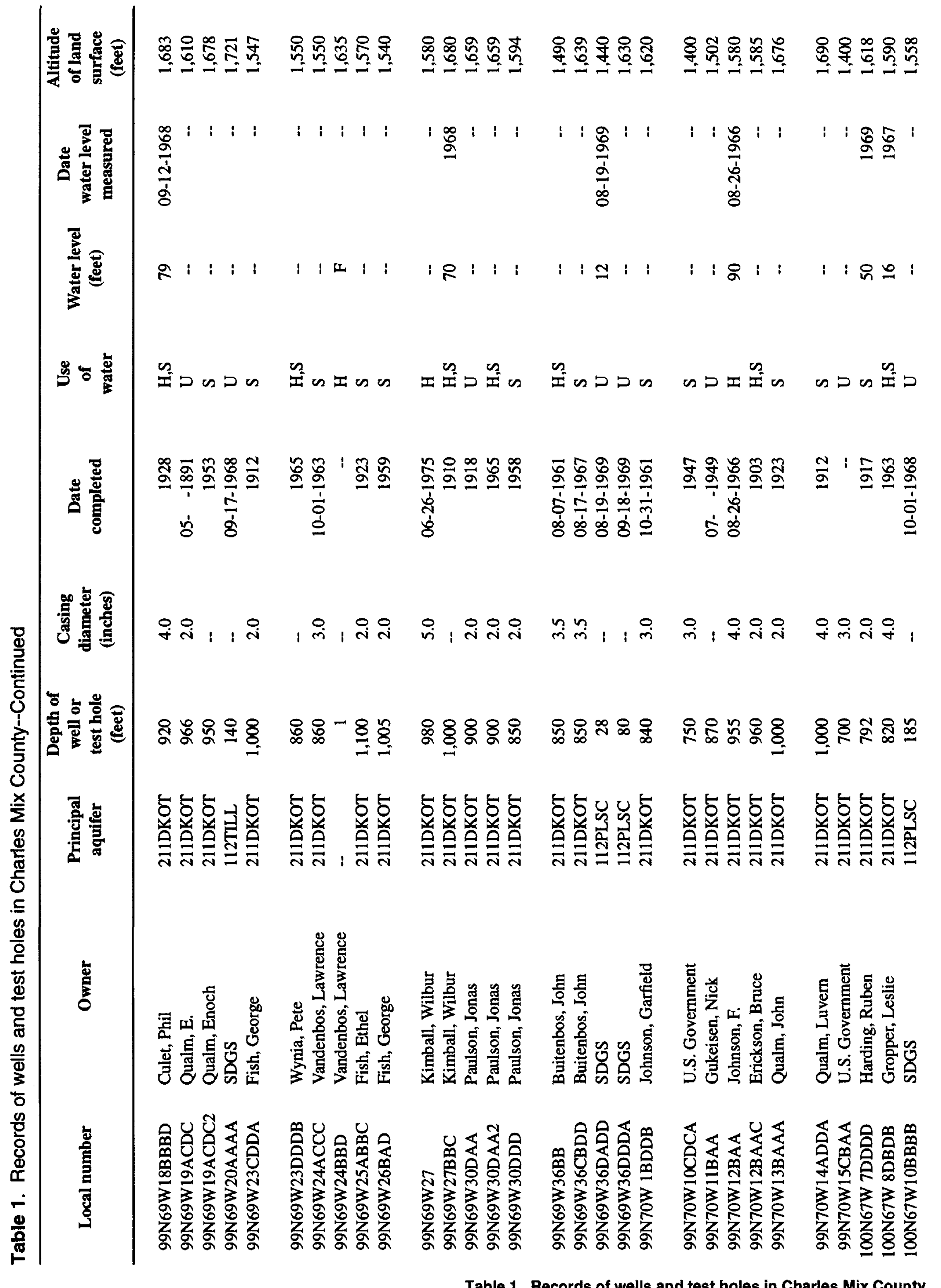

Table 1. Records of wells and test holes in Charles Mix County 65 


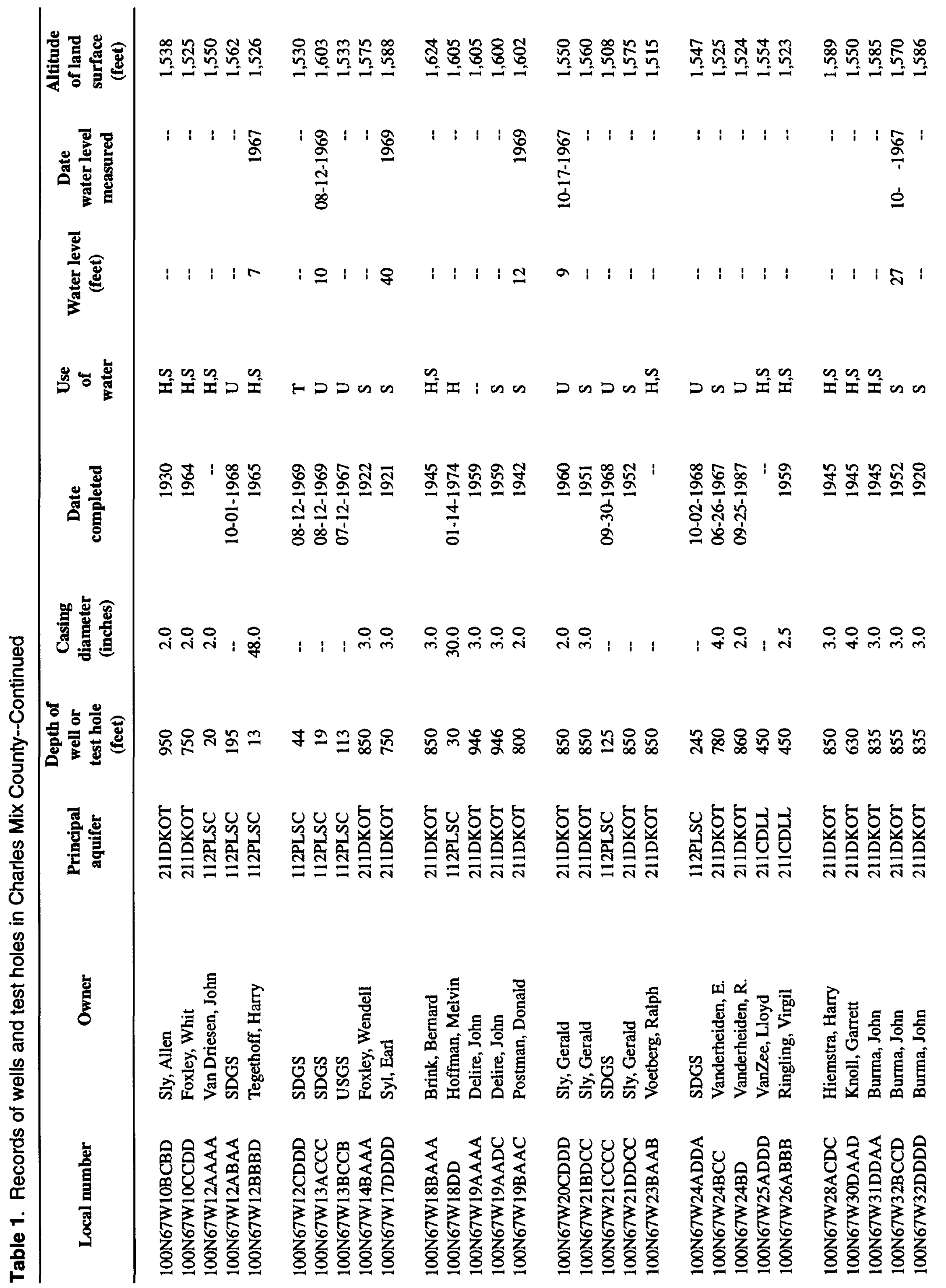

66 Records of Wells snd Chemicsl Analyses of Ground Water in Charles Mix and Douglas Counties, South Dakota 


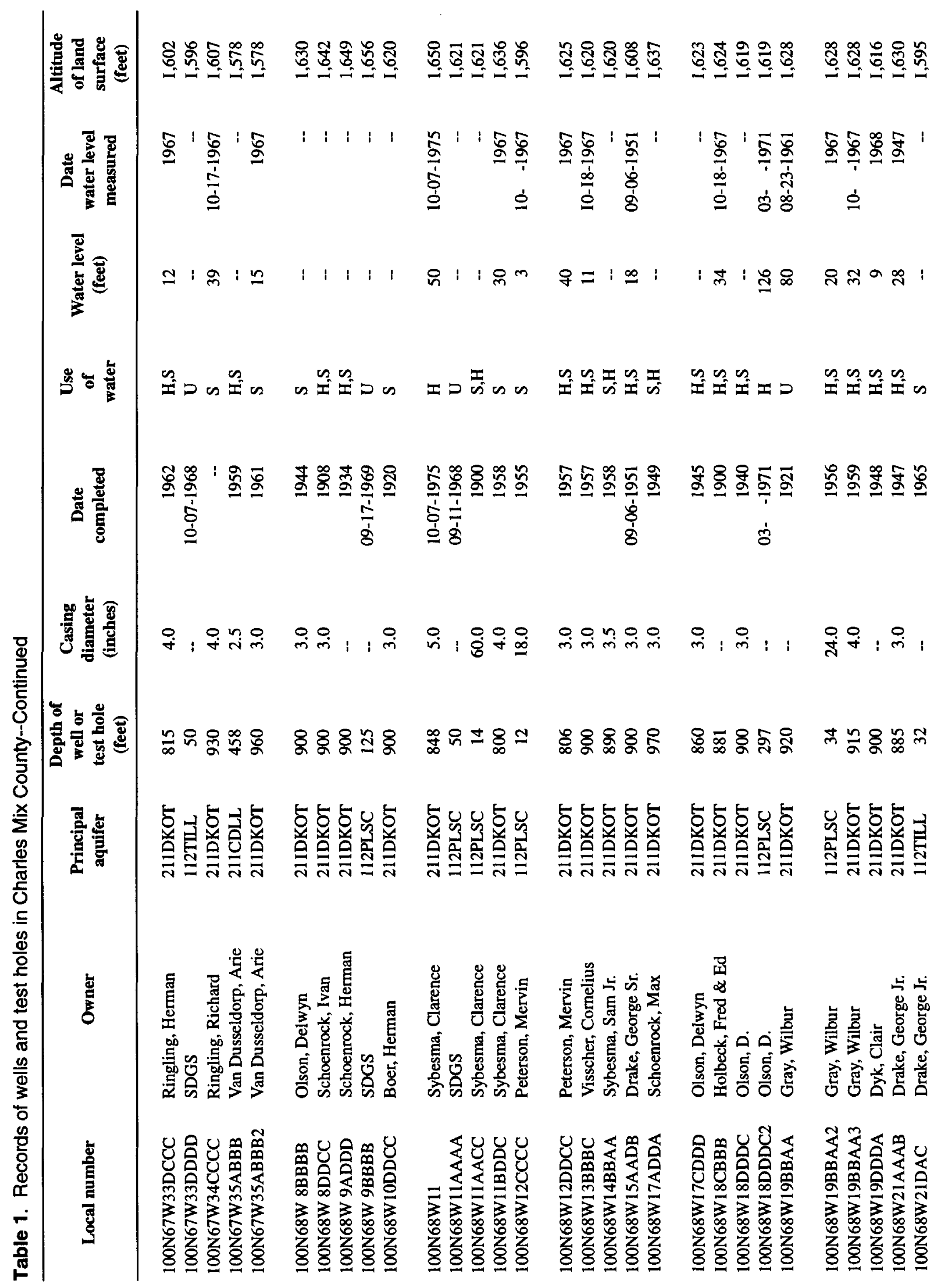

Table 1. Records of wells and test holes in Charles Mix County 67 


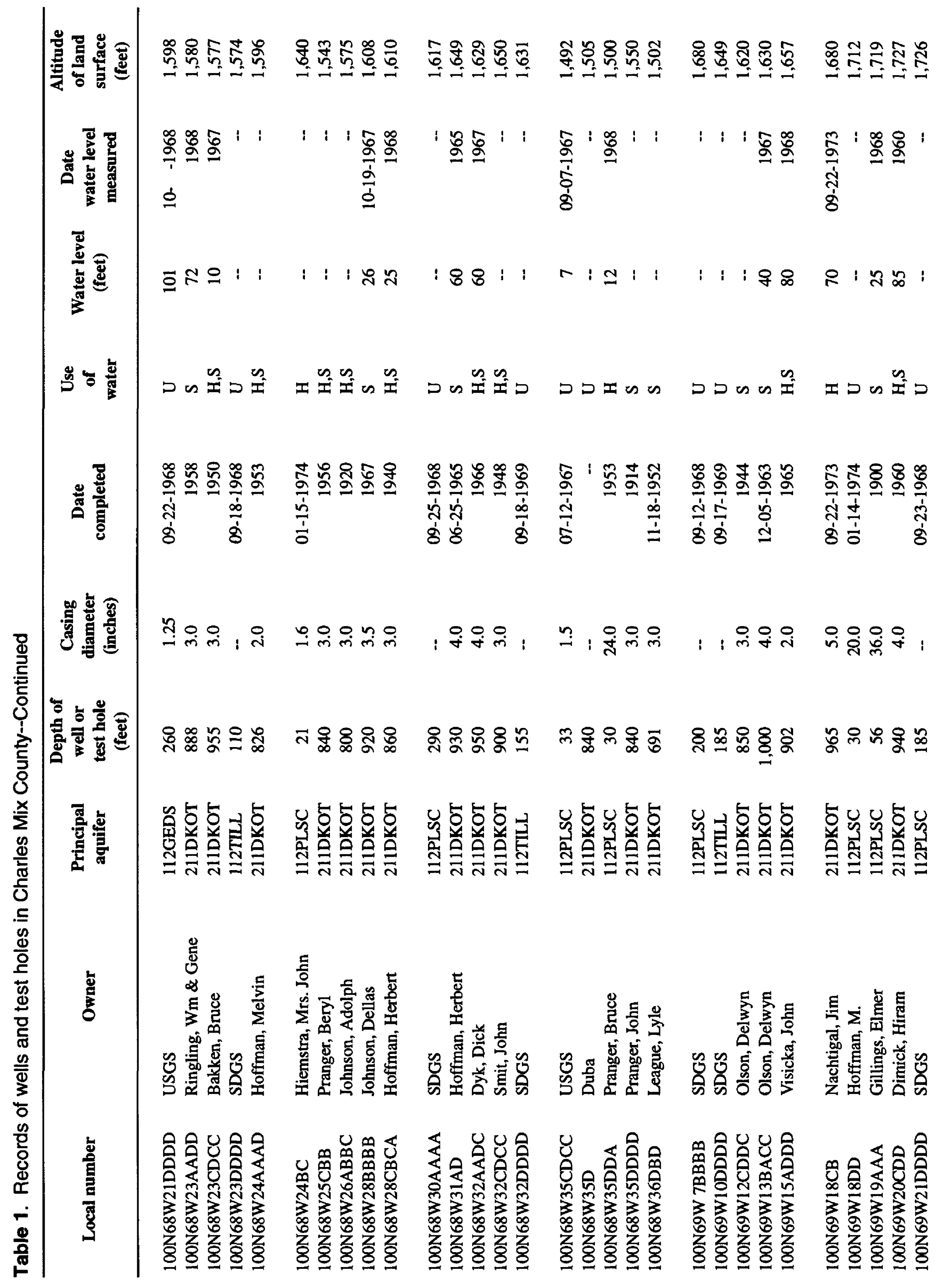

68 Records of Wells and Chemical Analyses of Ground Water In Chsrles Mix and Douglas Counties, South Dakota 


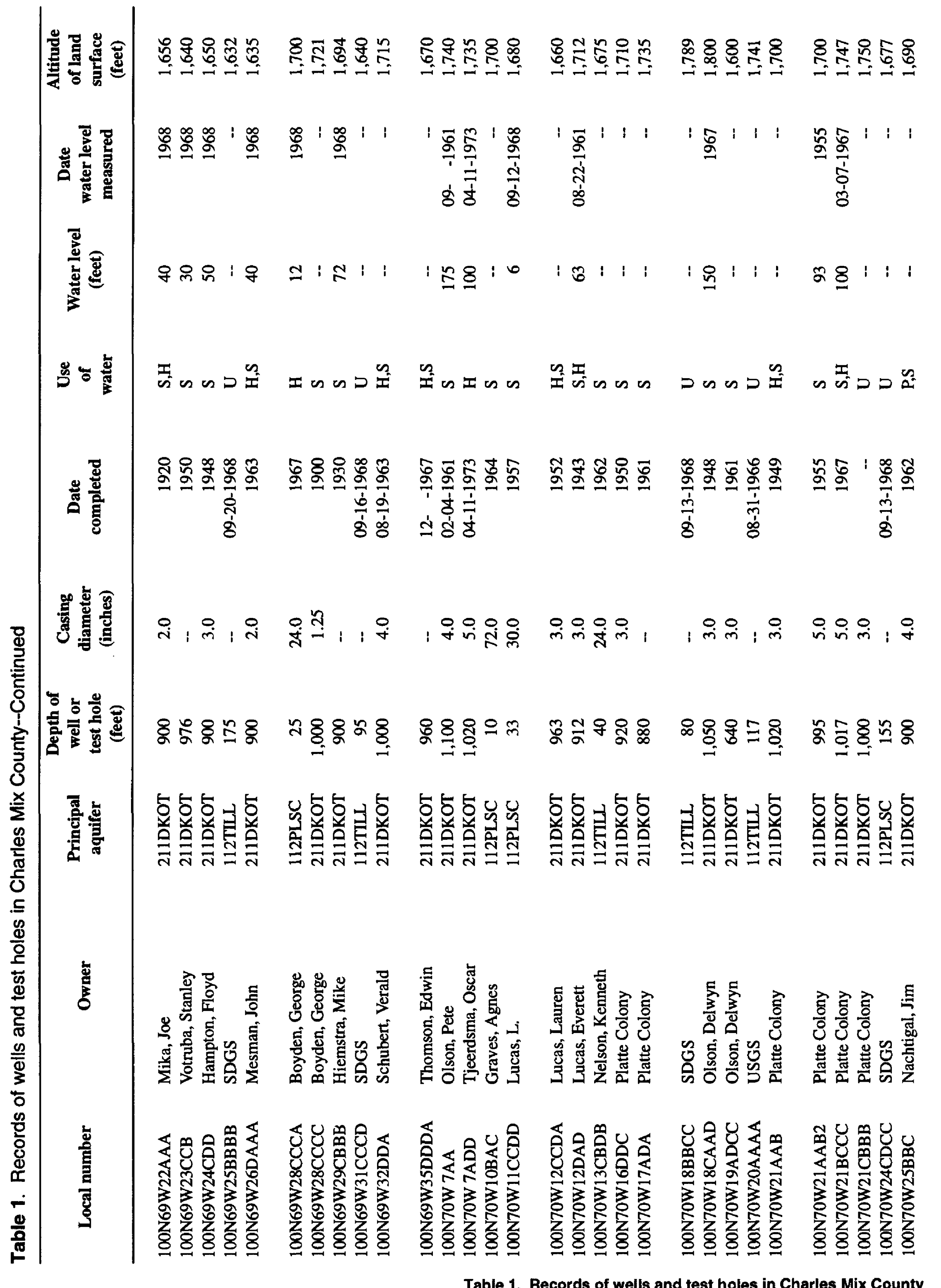




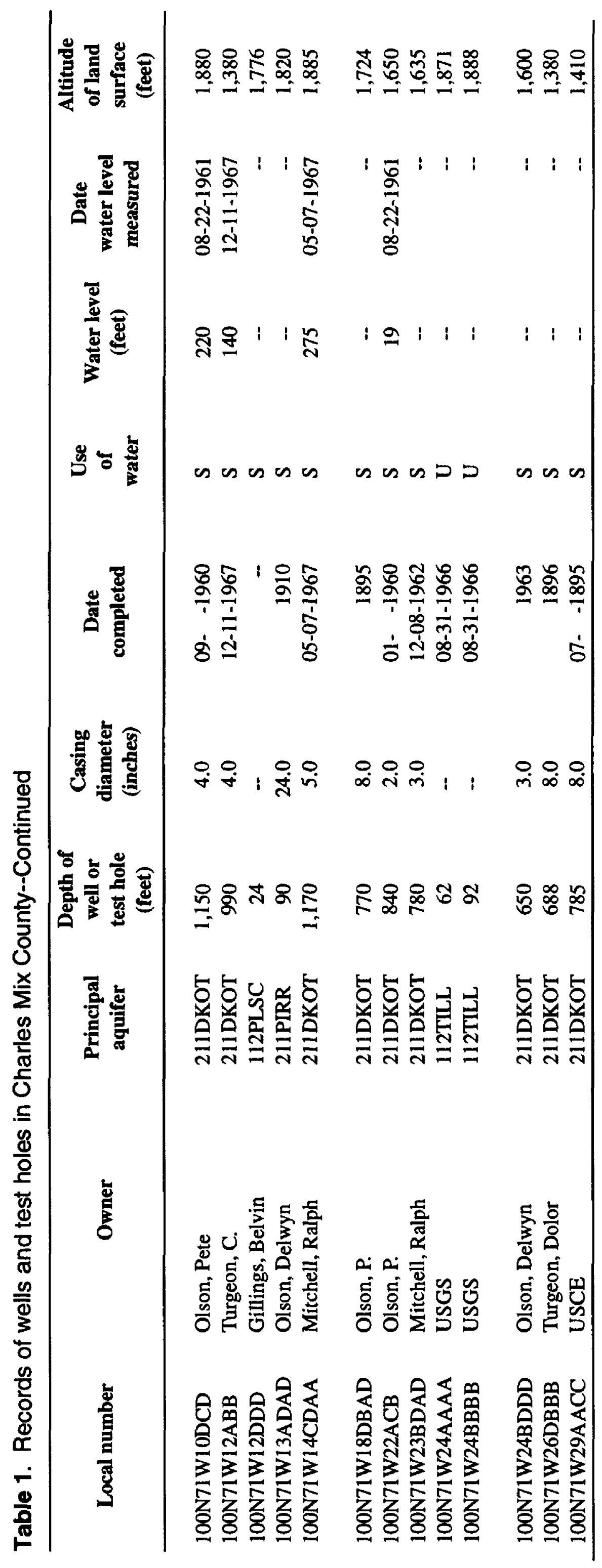

70 Records of Wells and Chemical Analyses of Ground Water in Cherles Mix end Douglas Counties, South Dakota 


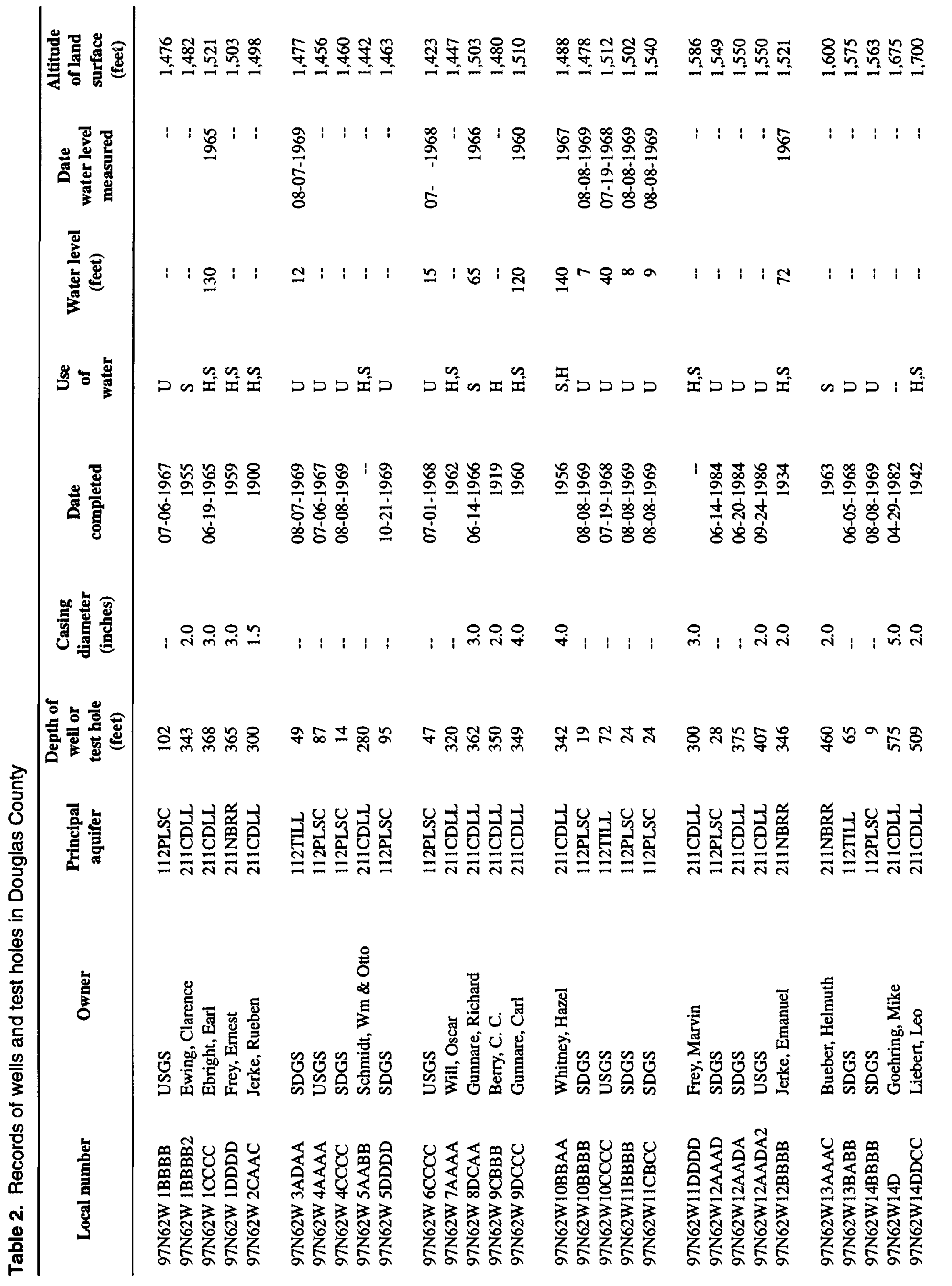




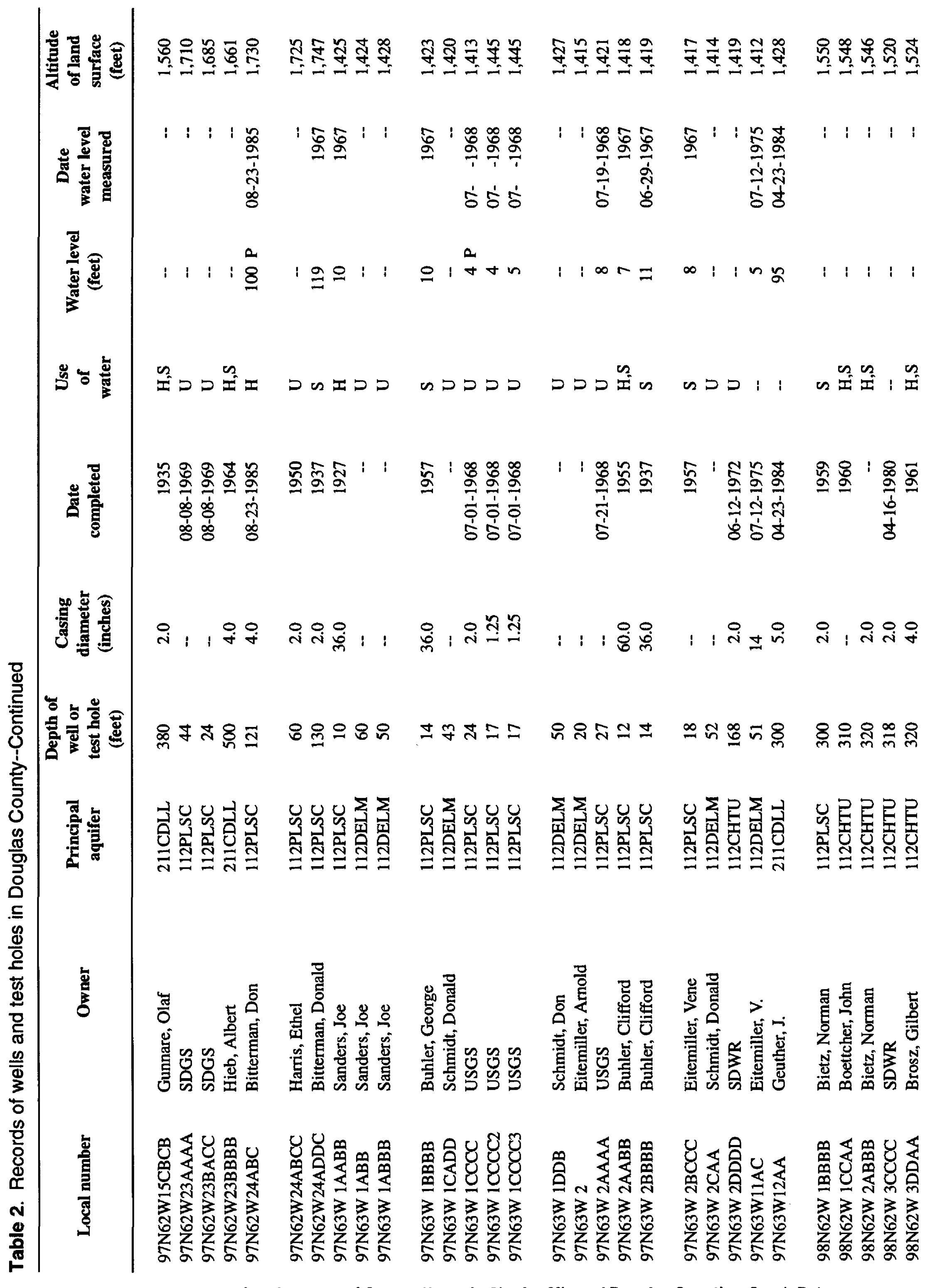

72 Records of Wells and Chemical Analyses of Ground Water in Charles Mix and Douglas Counties, South Dakota 


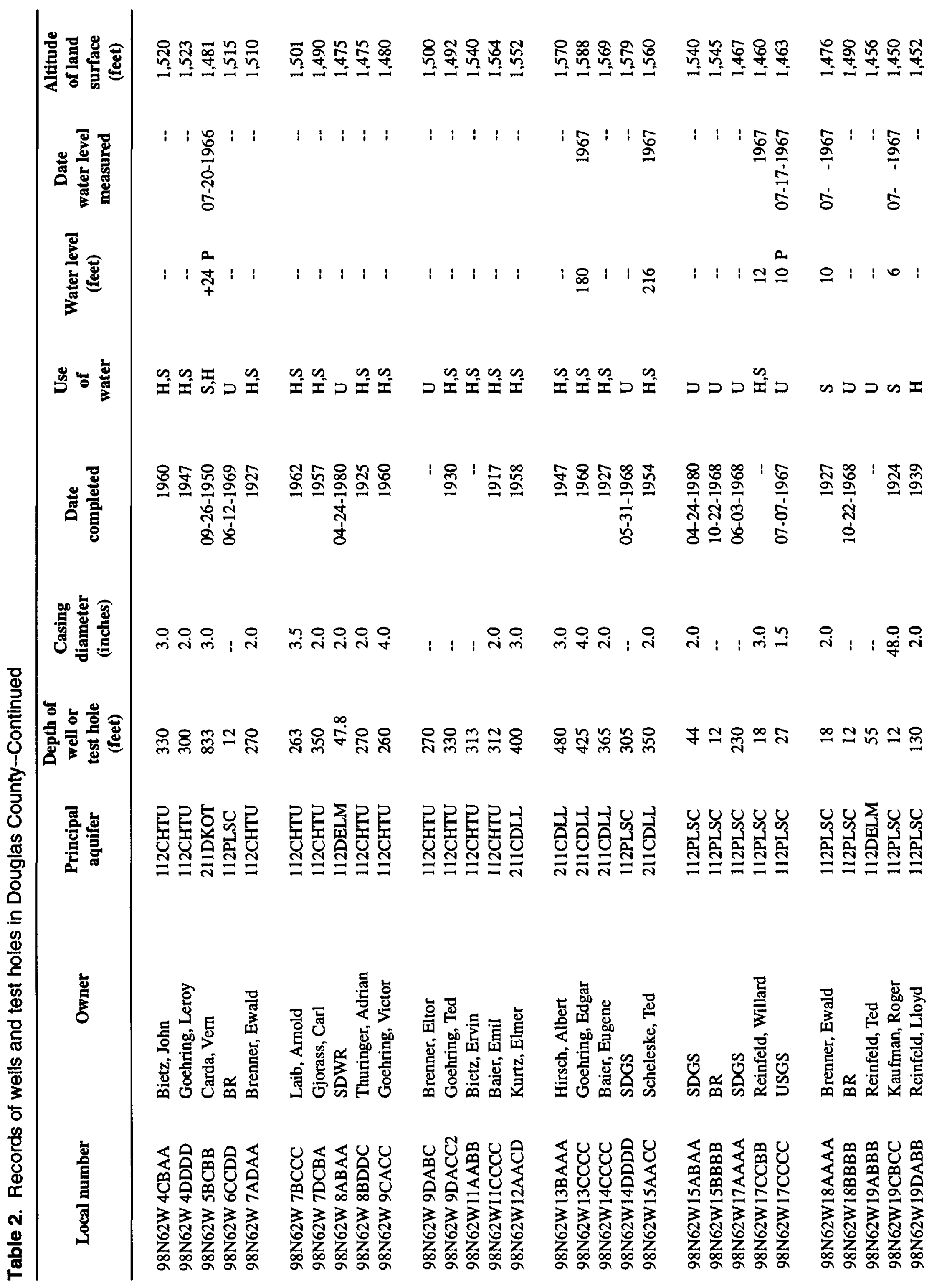




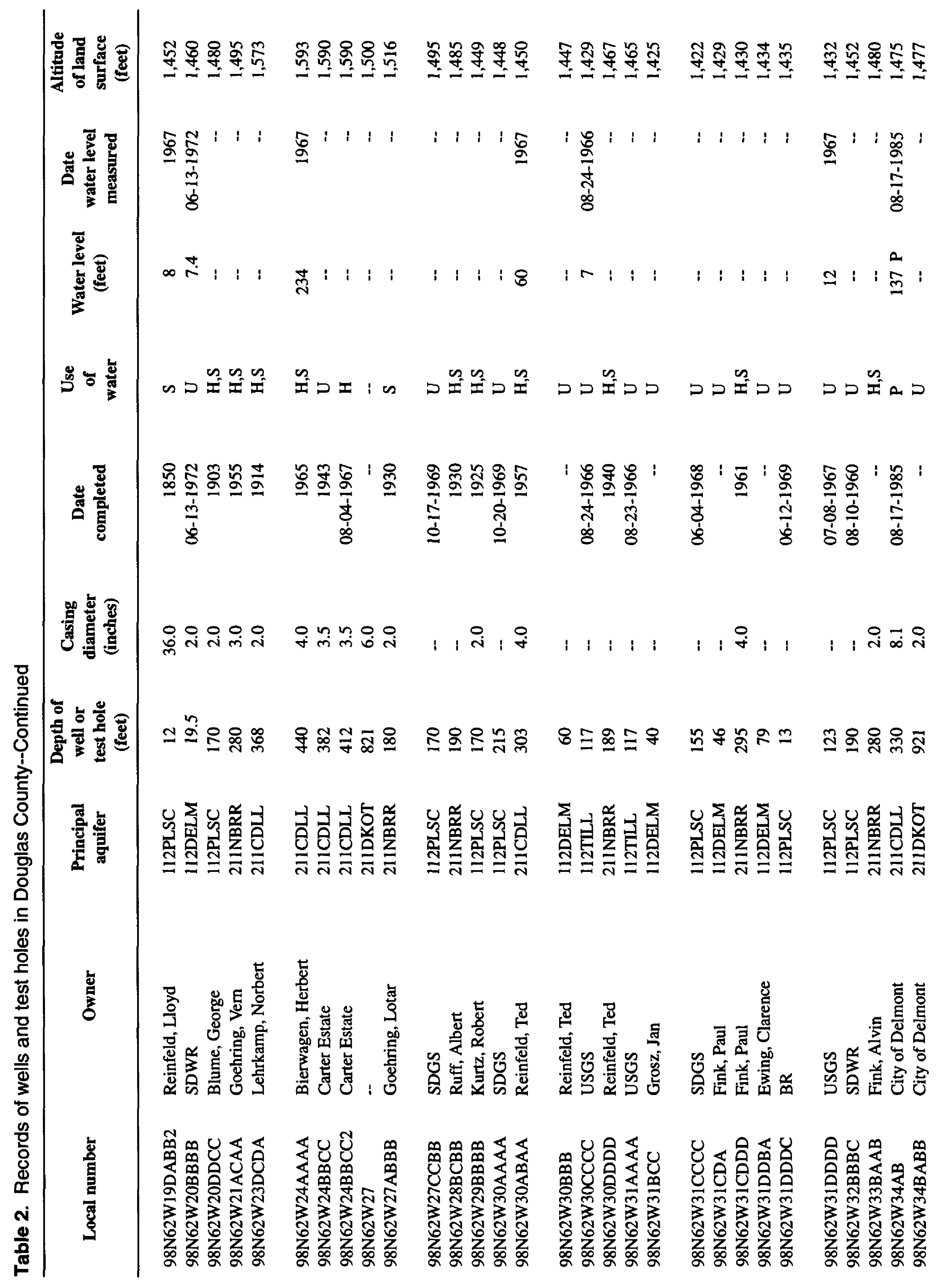




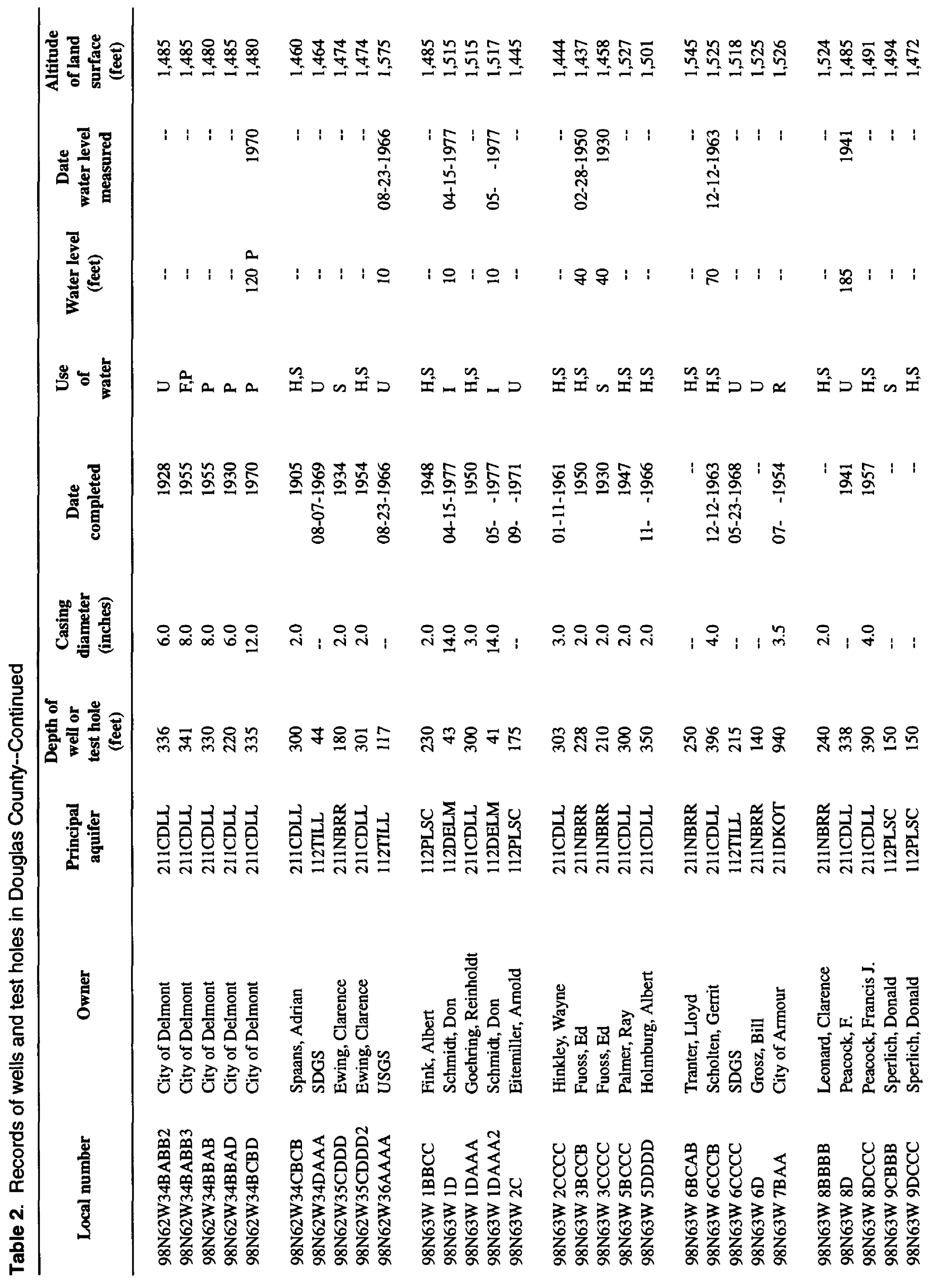




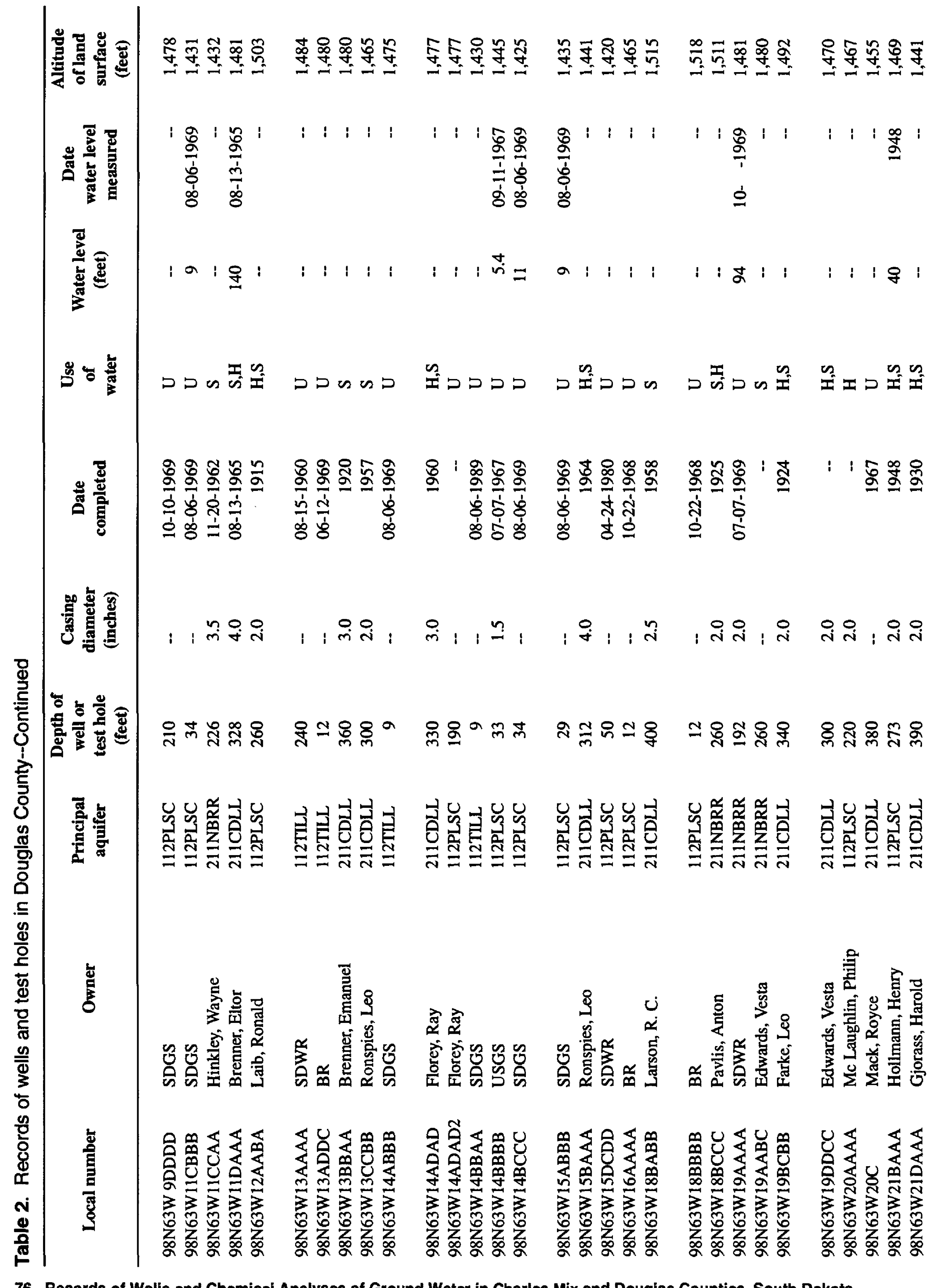

76 Records of Welis end Chemicei Analyses of Ground Water in Charles Mix end Dougias Counties, South Dekota 


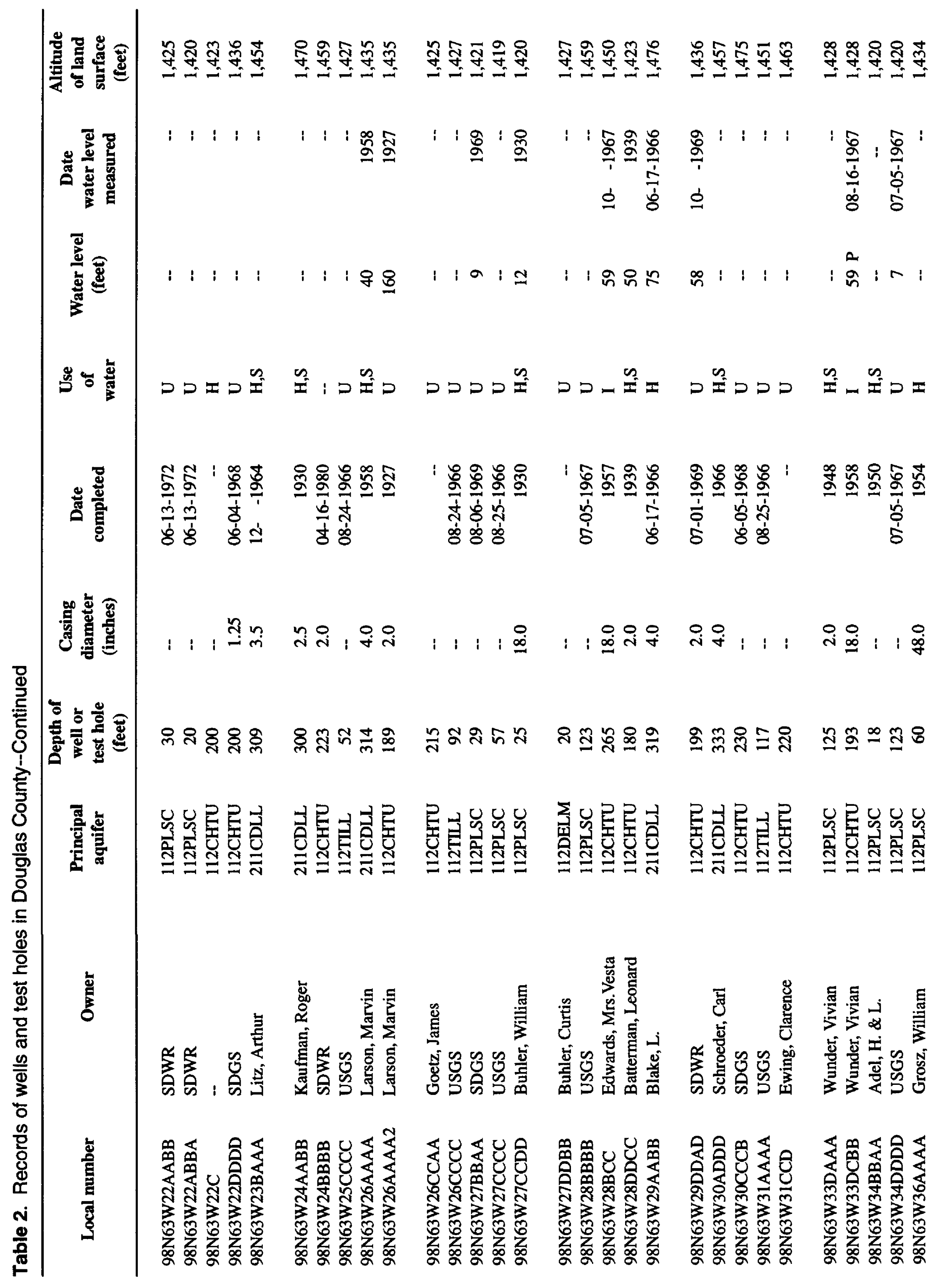

Table 2. Records of wells and test holes in Douglas County 77 


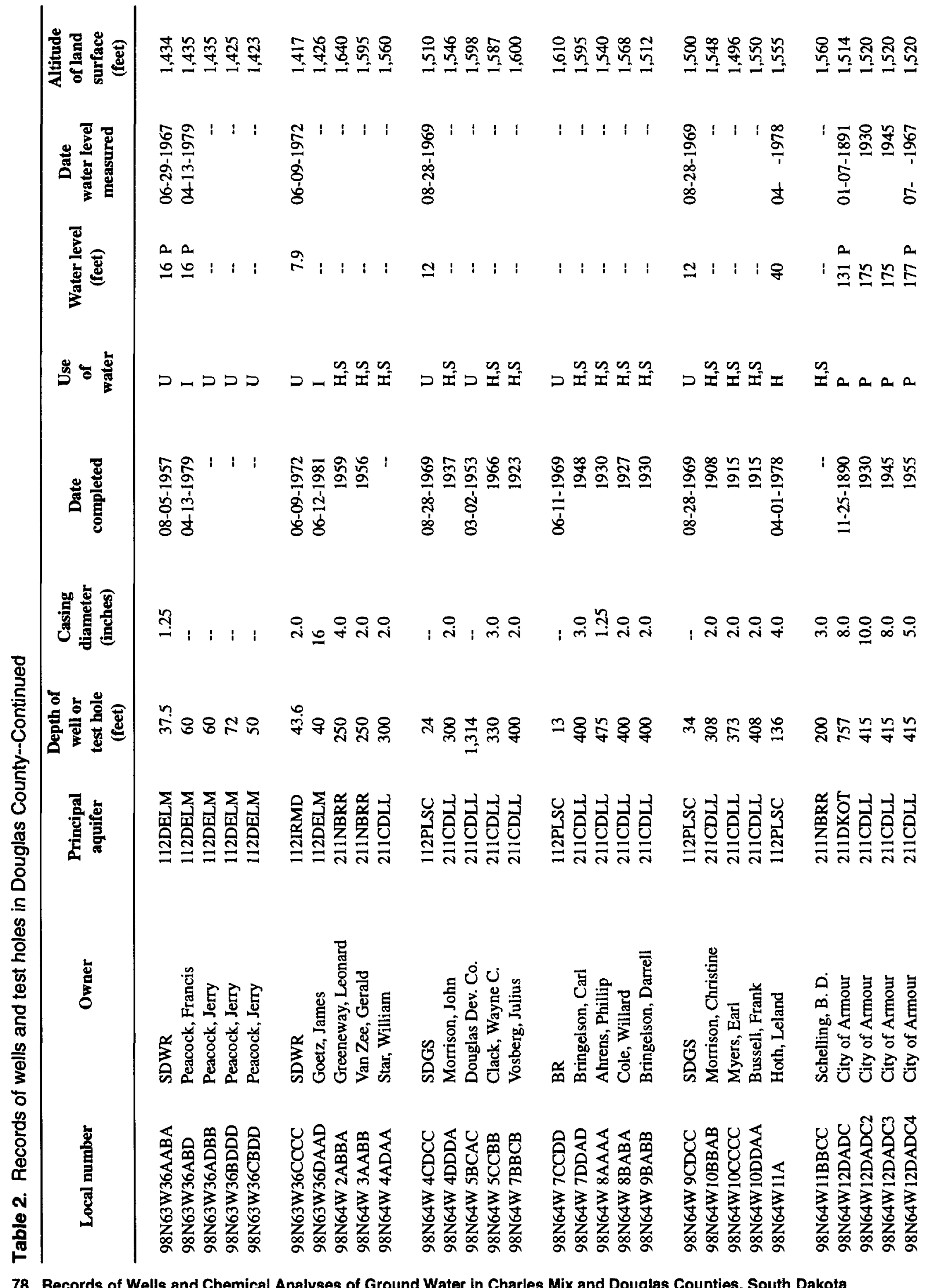




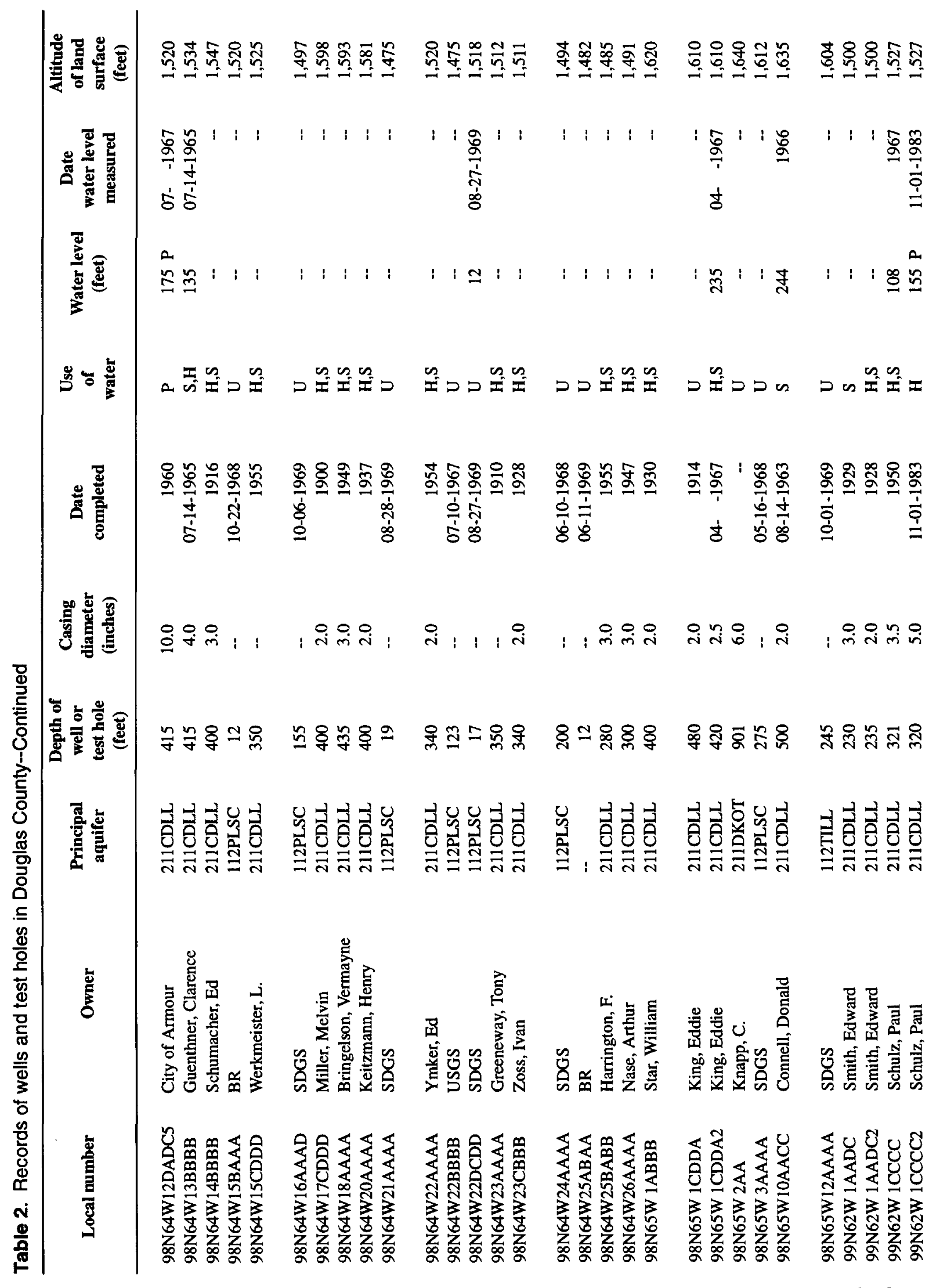




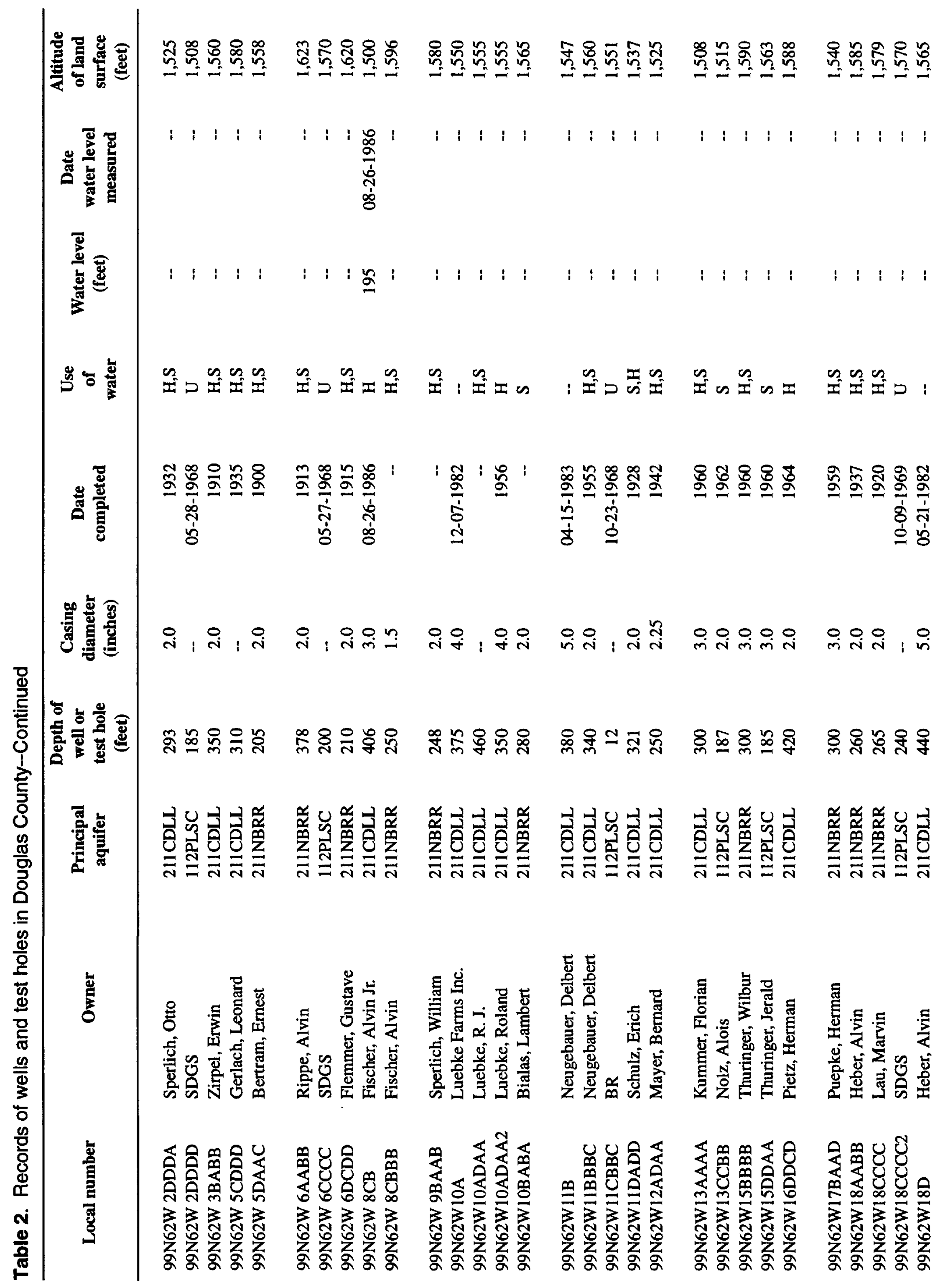

80 Records of Wells and Chemlcal Anelyses of Ground Water in Charles Mix and Douglas Counties, South Dakota 


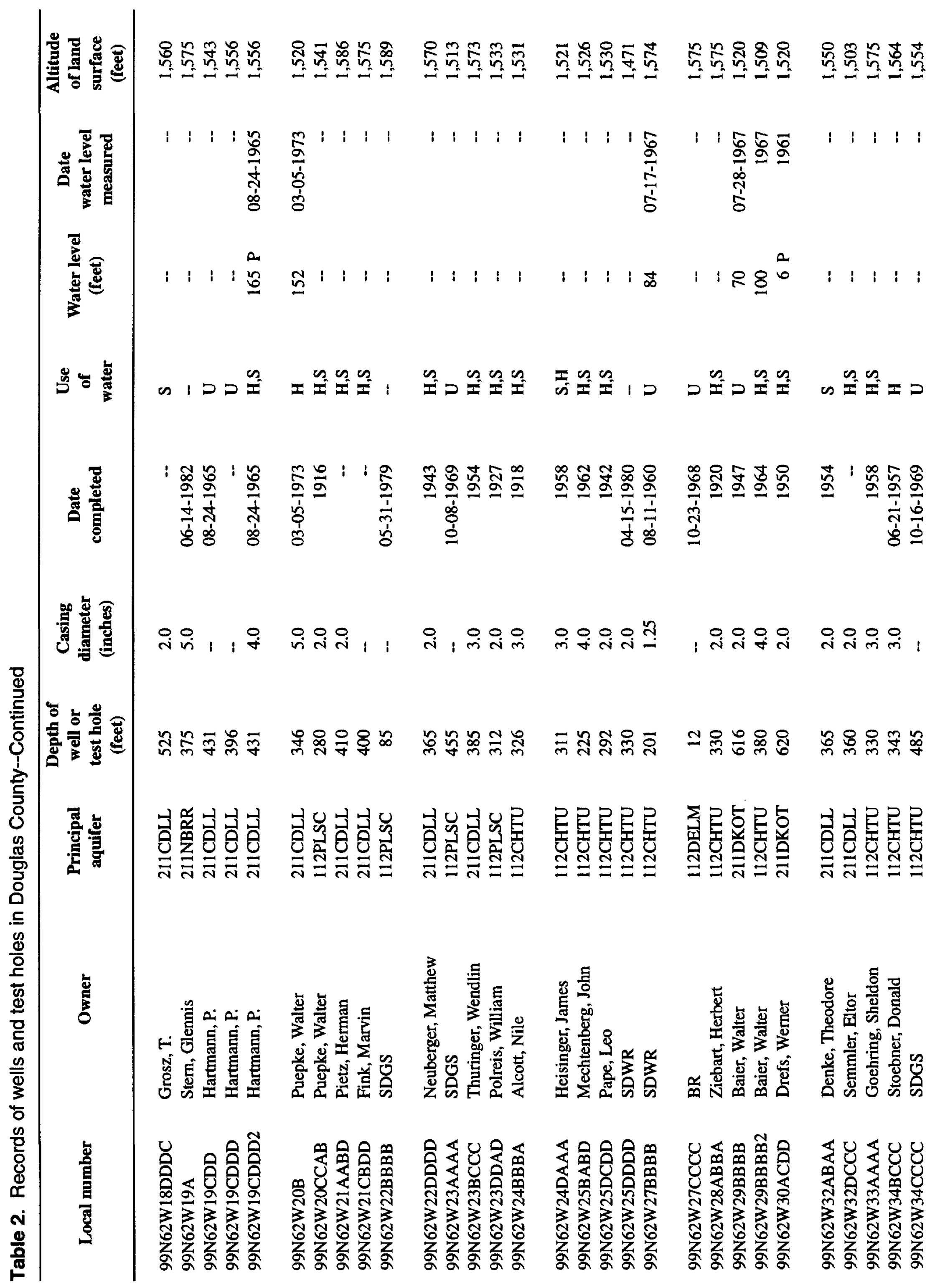




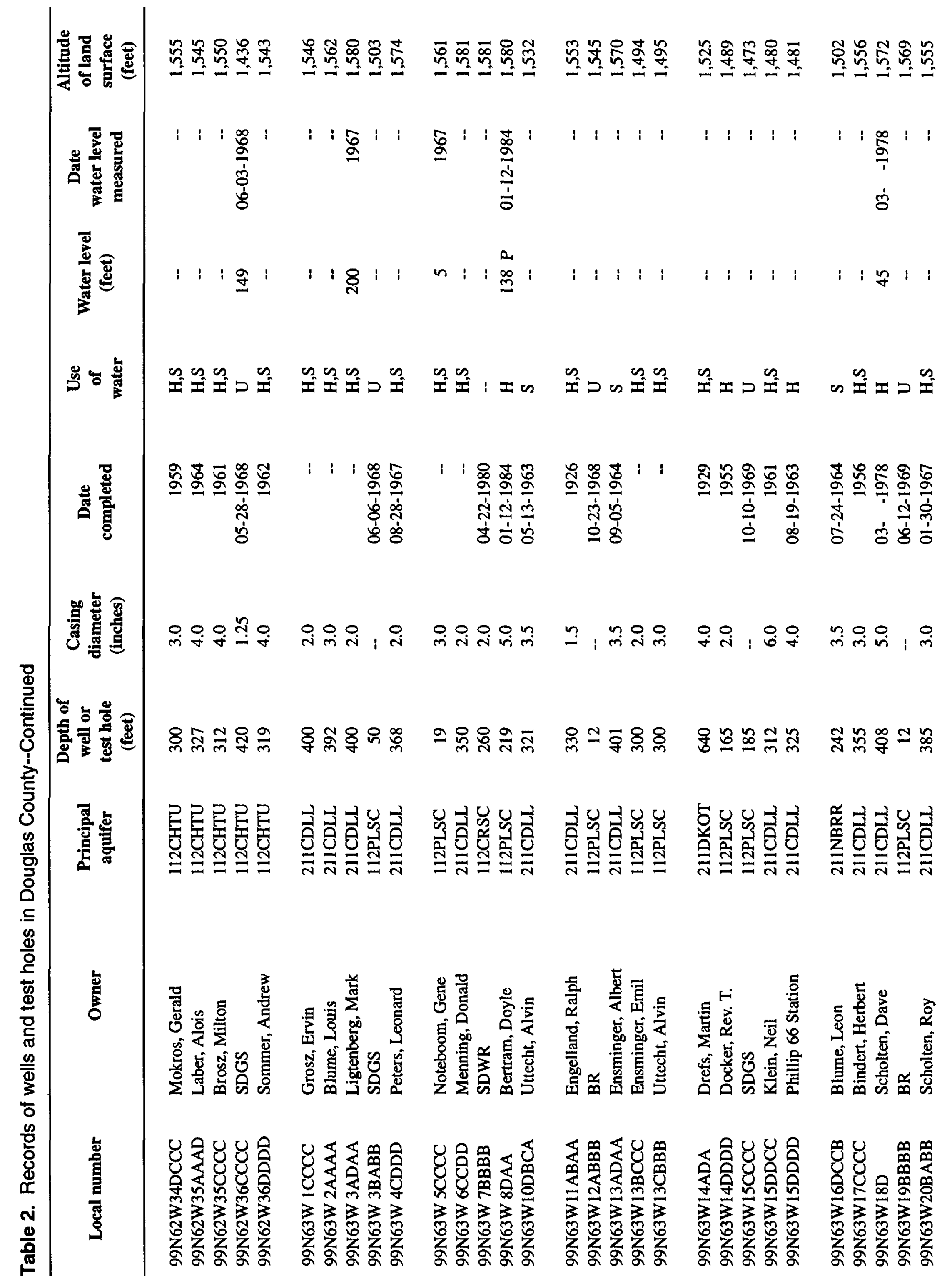

82 Records of Wells and Chemical Anslyses of Ground Water in Charles Mix and Douglas Countles, South Dakota 


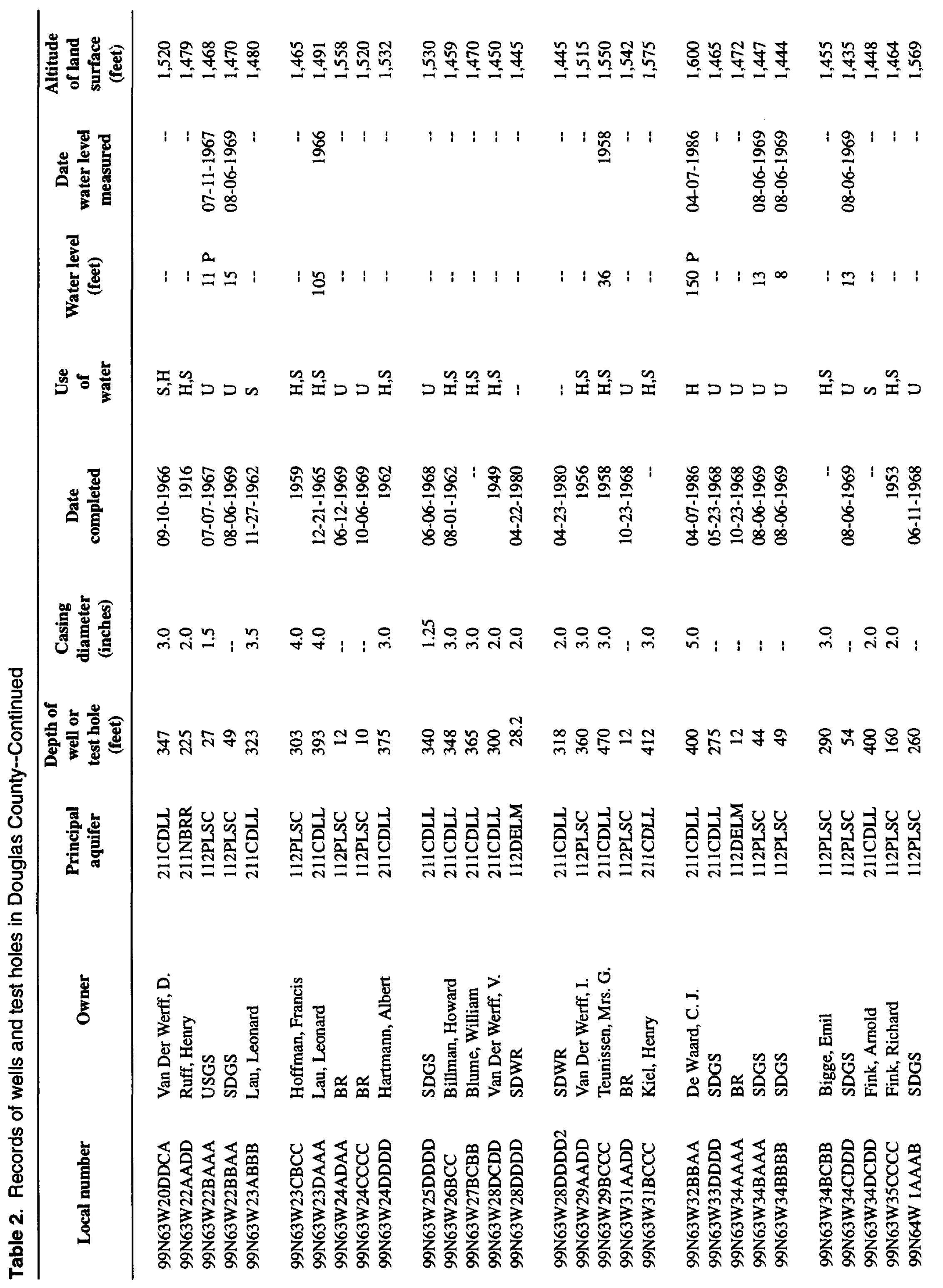




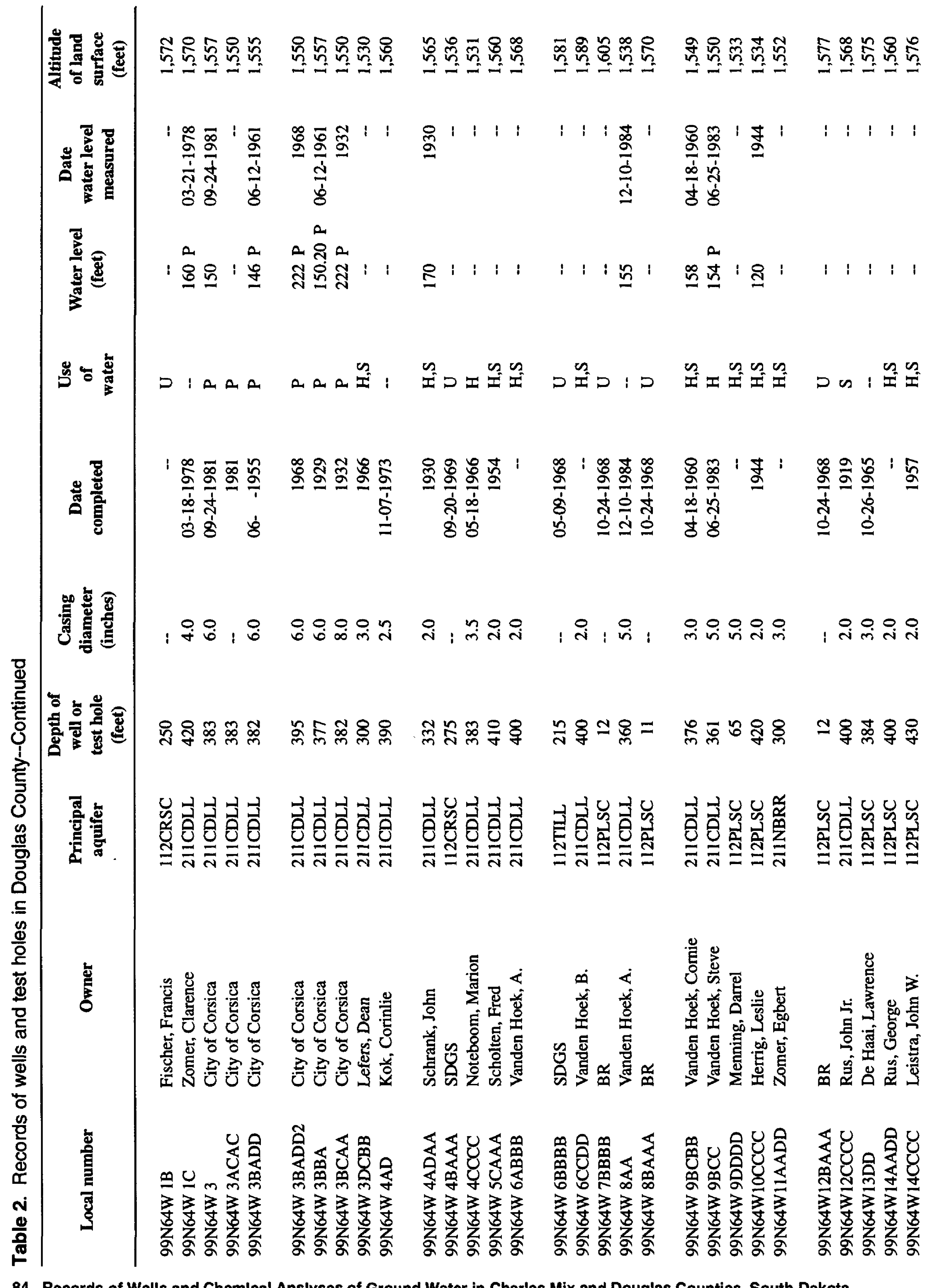

84 Records of Wells and Chemlcal Anslyses of Ground Water in Charles Mix and Douglas Counties, South Dakota 


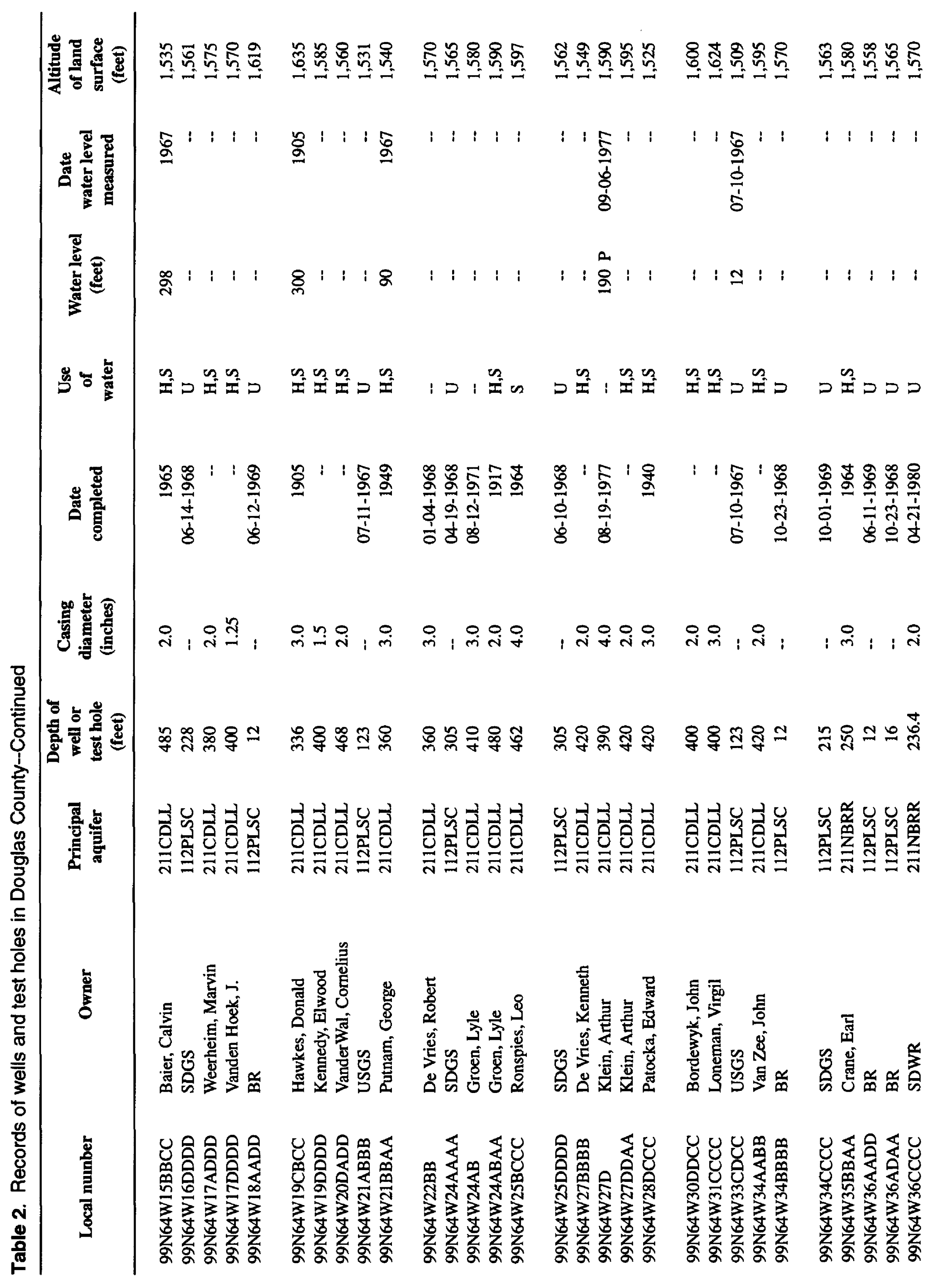




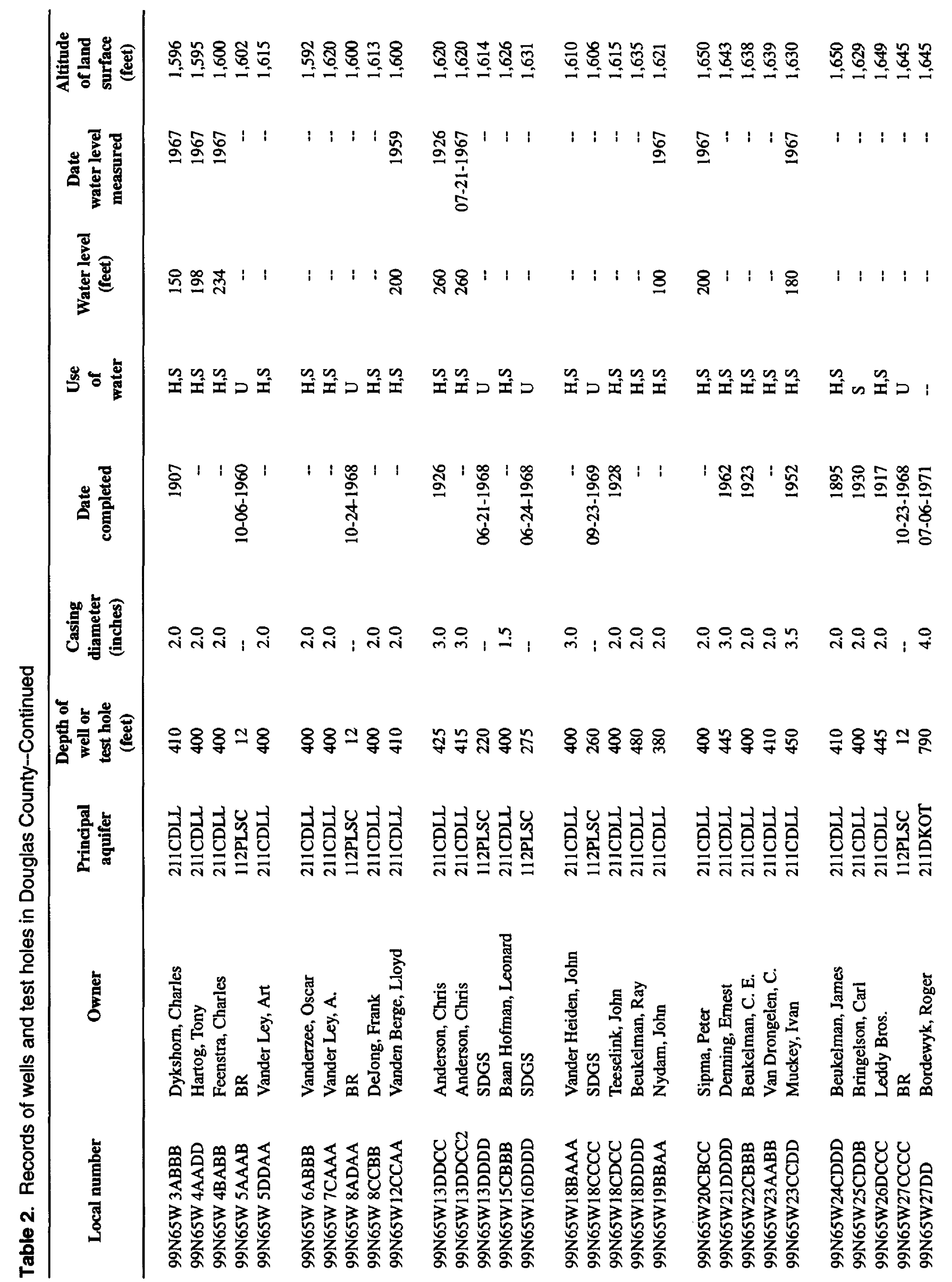




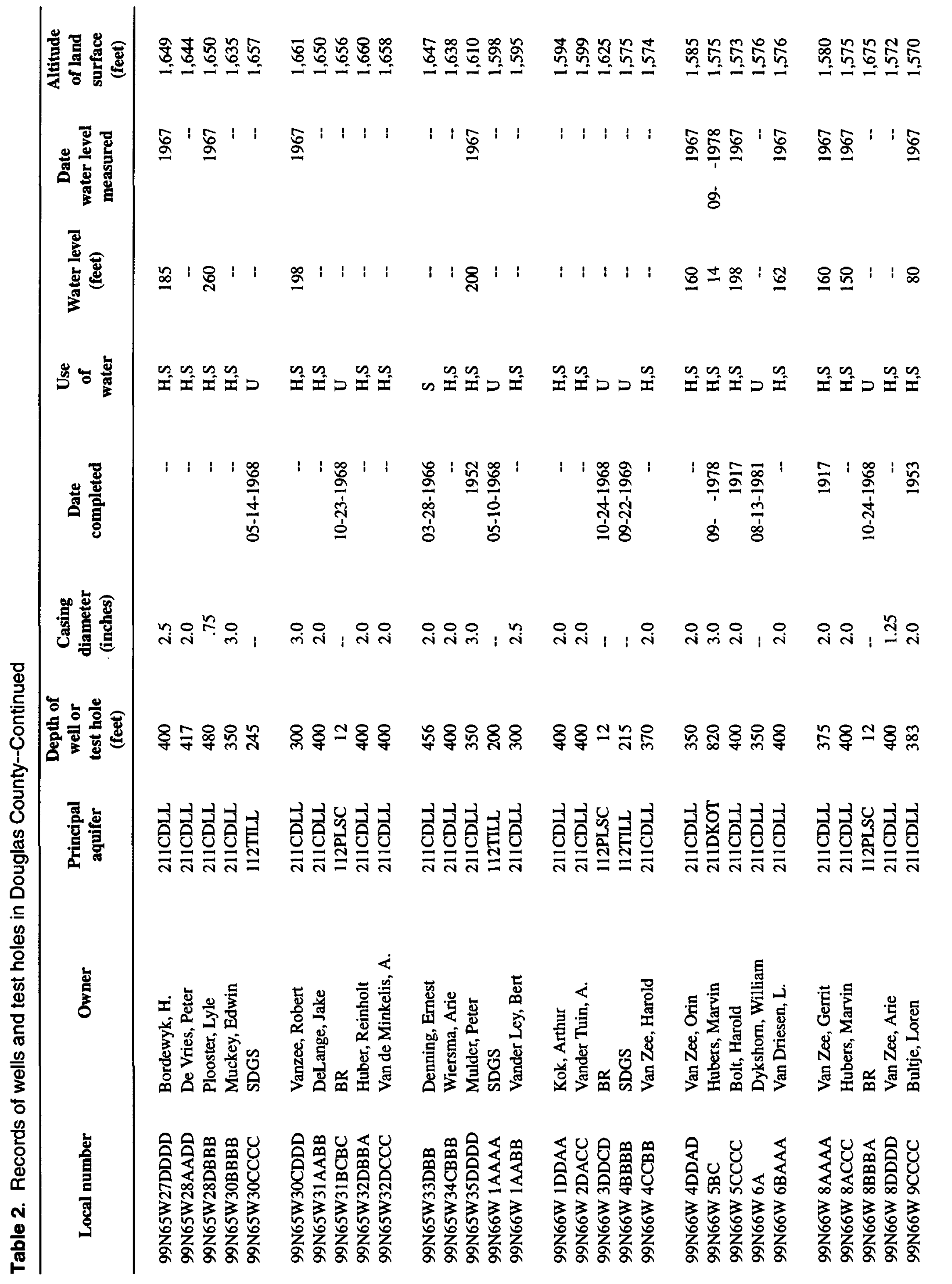




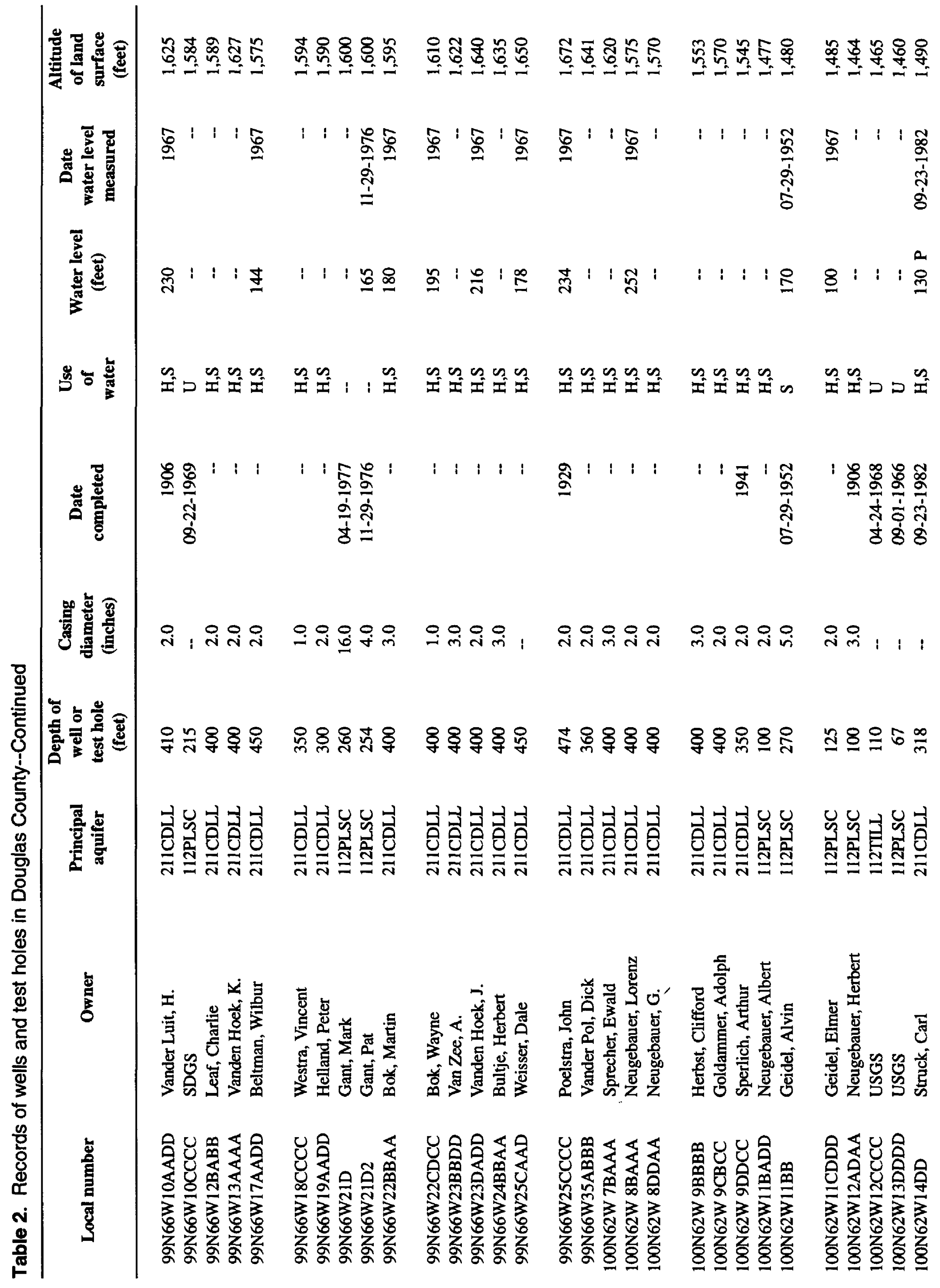

88 Records of Wells and Chemical Analyses of Ground Water in Chsrles Mix and Douglas Countles, South Dakota 


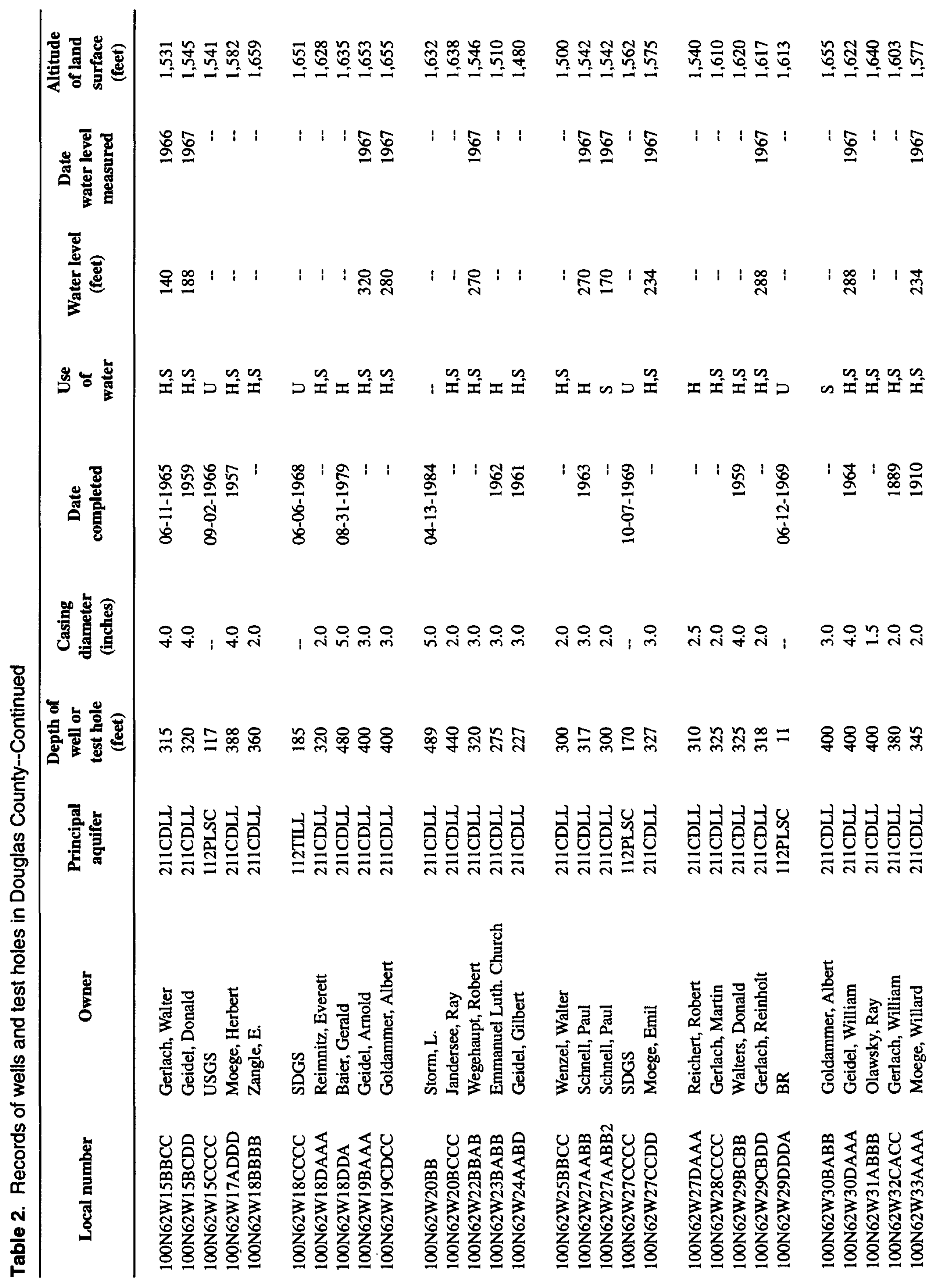




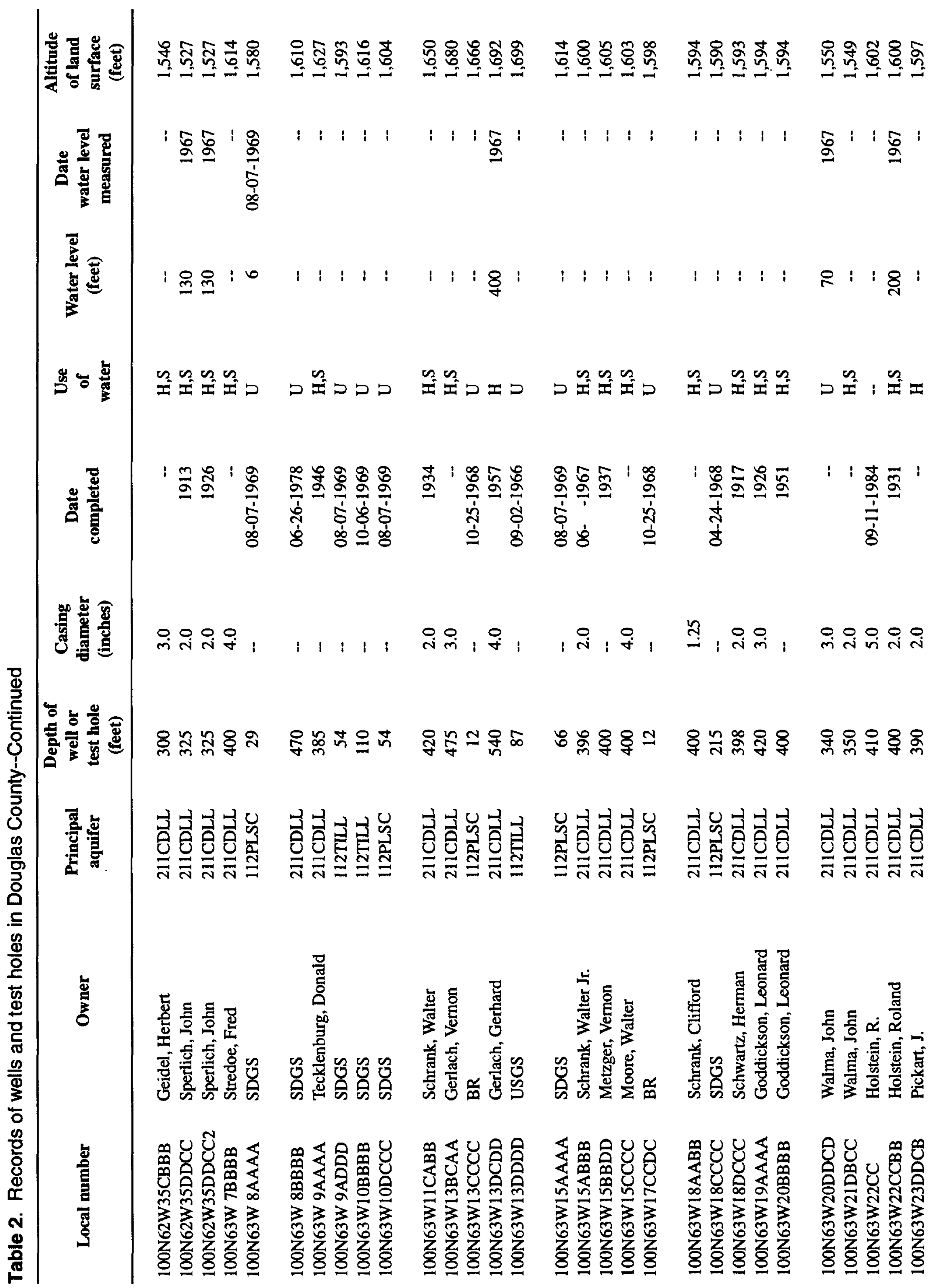

90 Records of Wells and Chemlcal Anslyses of Ground Water in Charles Mix and Douglas Counties, South Dakota 


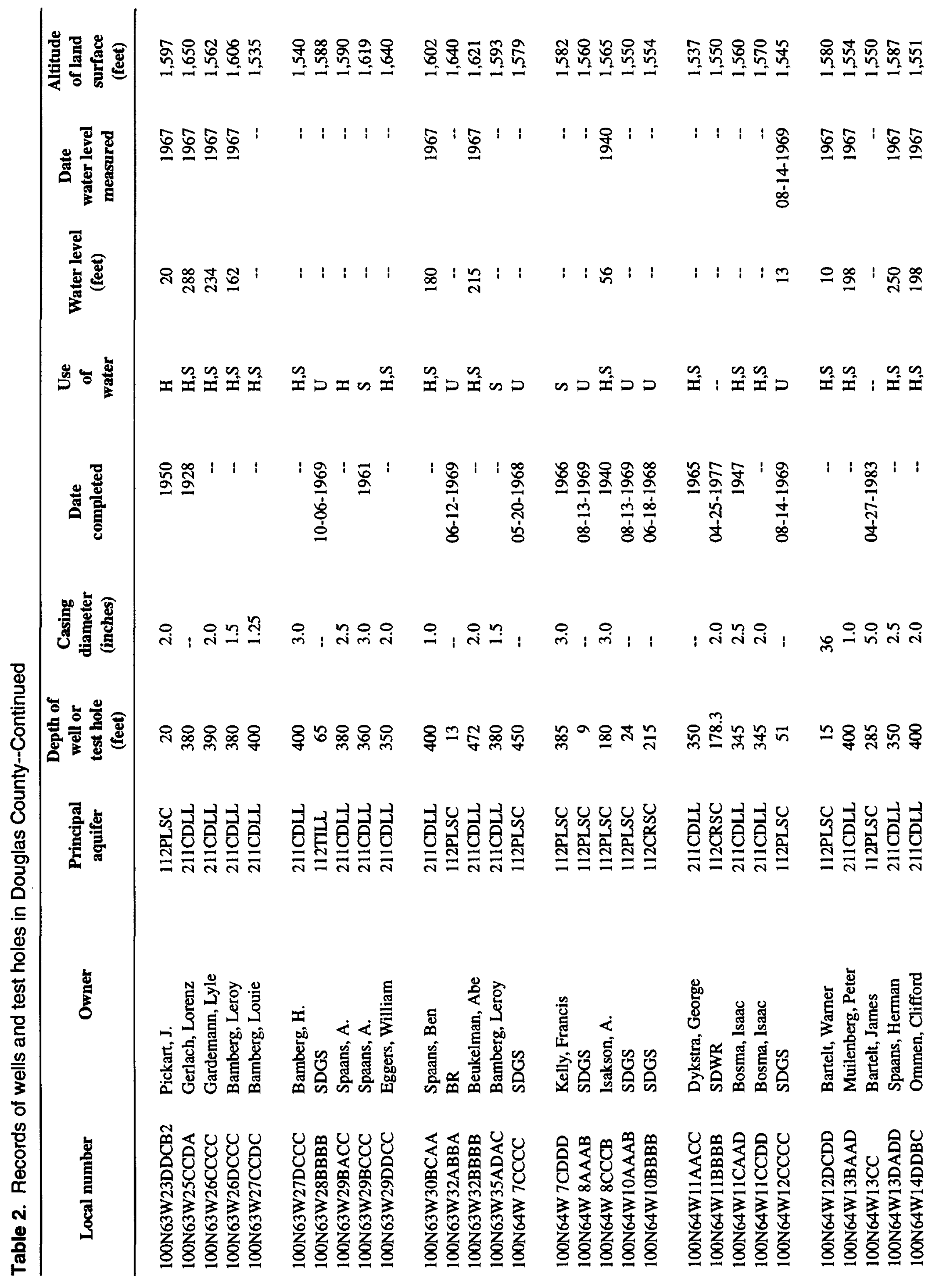

Table 2. Records of wells and test holes in Douglas County 91 


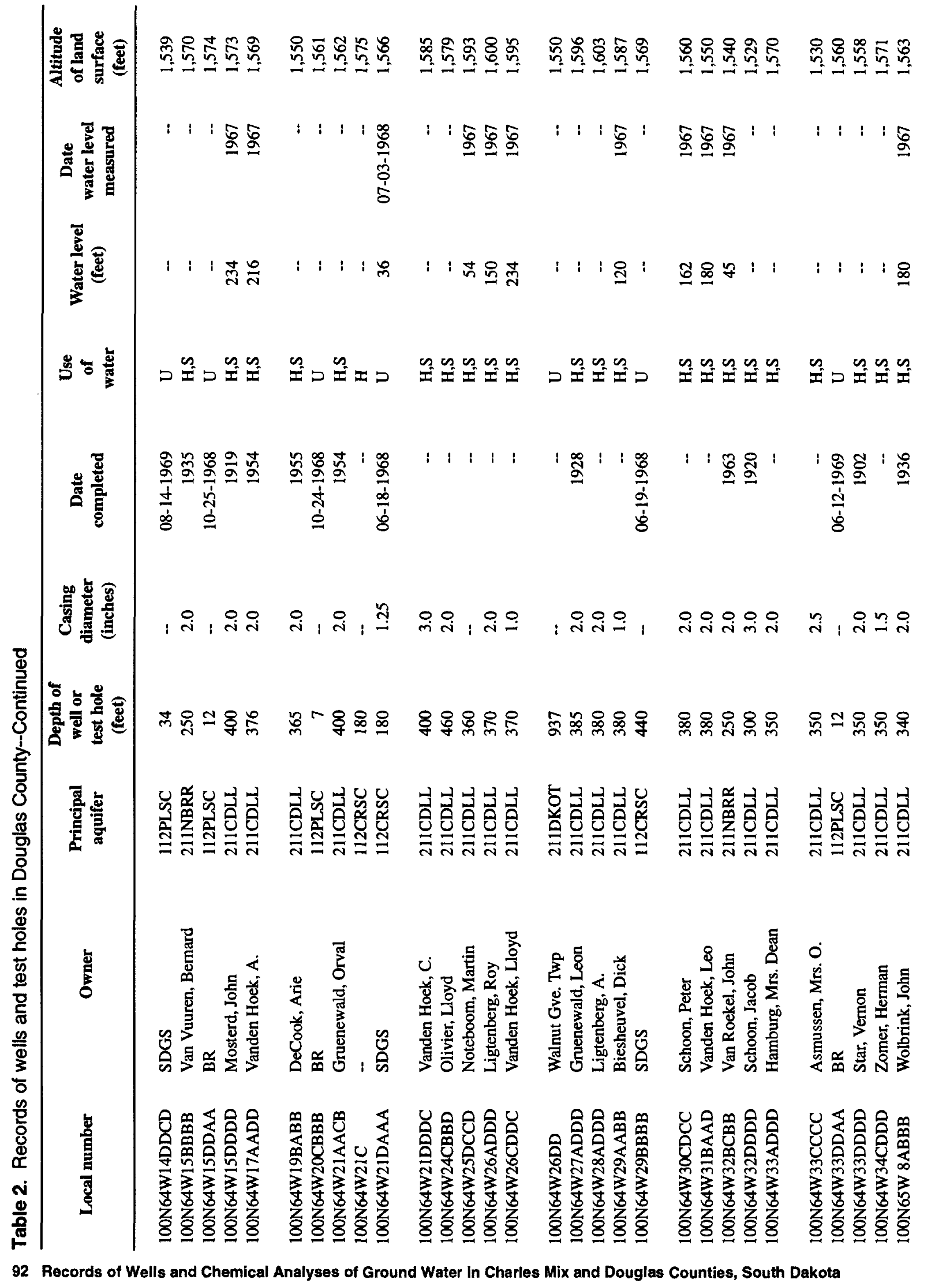




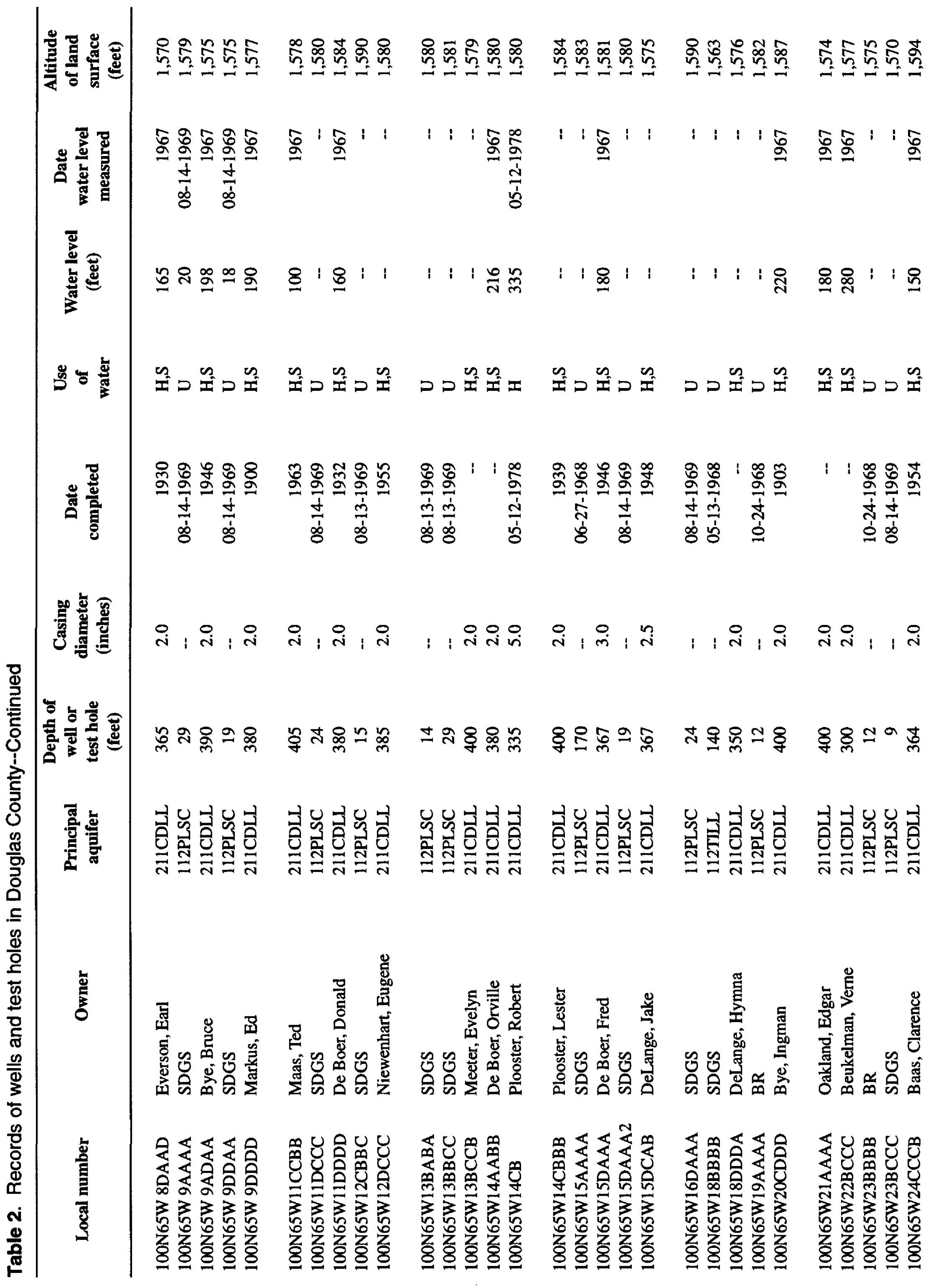




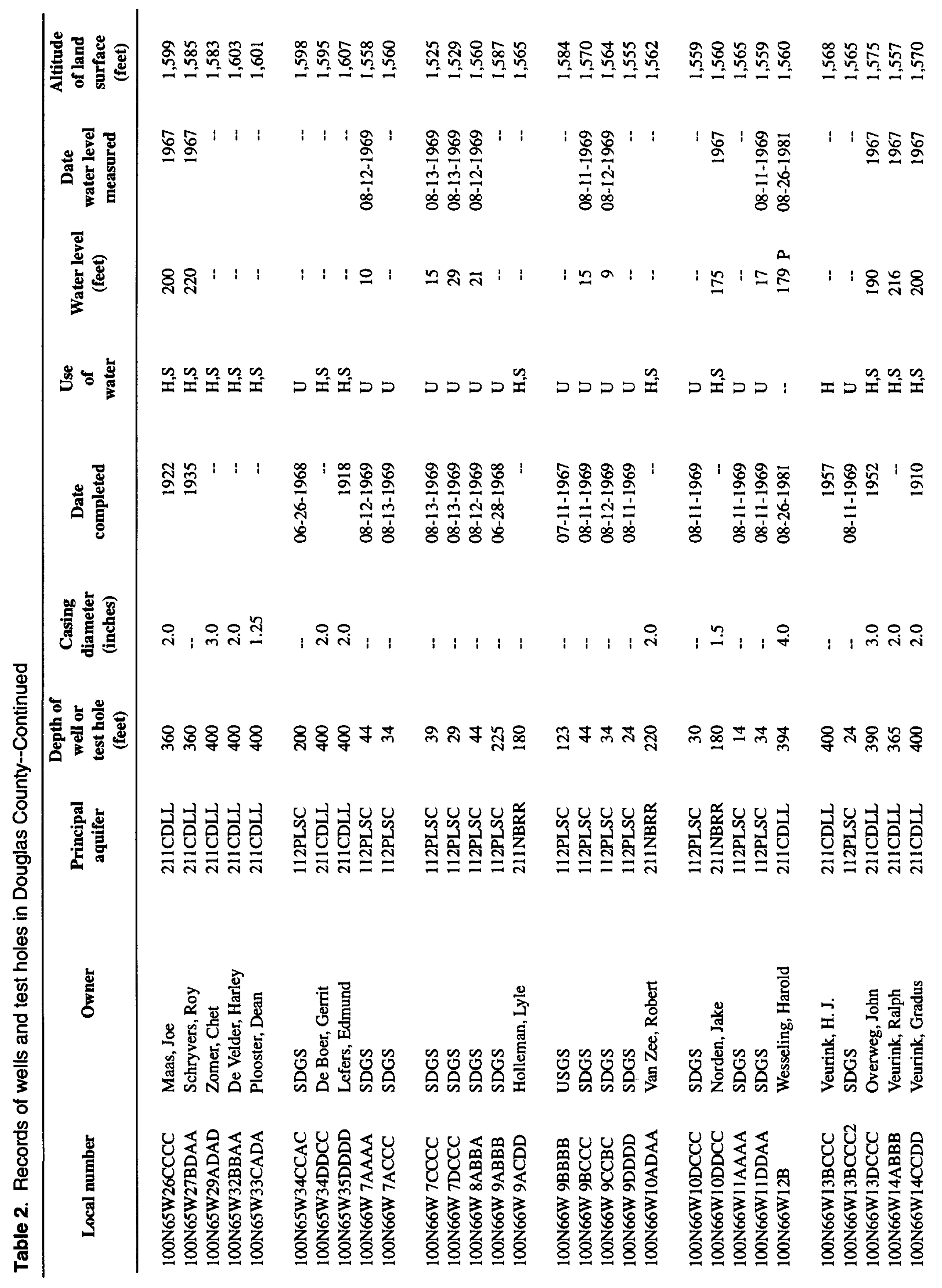

94 Records of Wells and Chemical Analyses of Ground Water in Charles Mix and Douglas Counties, South Dakota 


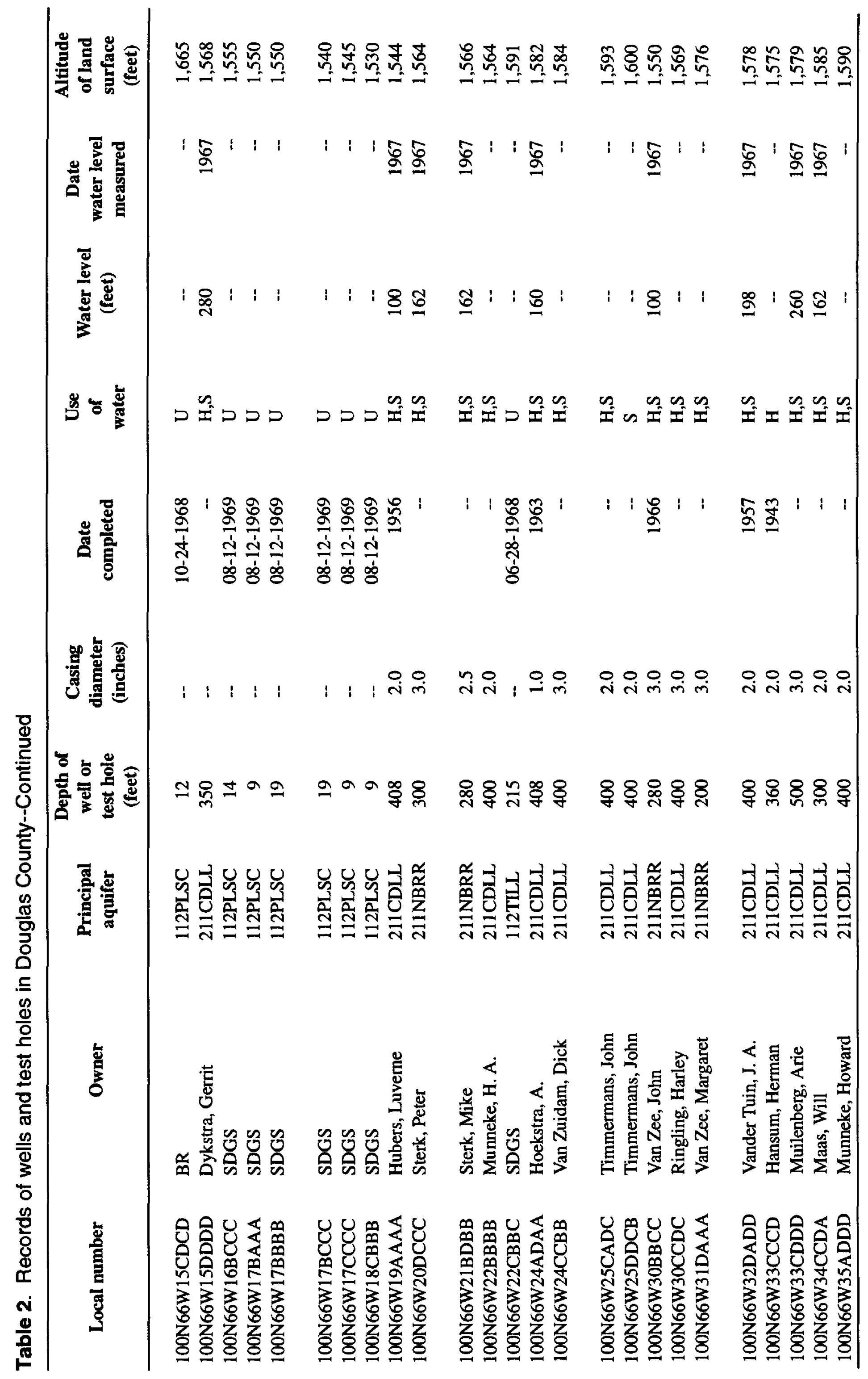


Blour pons

60 


\section{Explanation}

The following abbreviations and codes are used in table 3 :

${ }^{\circ} \mathrm{C}$ - degree Celsius

$\mu \mathrm{S} / \mathrm{cm}$ - microsiemens per centimeter at $25^{\circ} \mathrm{Celsius}$

$\mathrm{mg} / \mathrm{L}$ - milligrams per liter

$\mu \mathrm{g} / \mathrm{L} \quad$ - micrograms per liter

FET-FLD- fixed end-point titration-field value

ND - not detected

$<\quad-$ less than

$>\quad-$ greater than

-- $\quad$ no data

Agency Collecting or Analyzing

810 - U.S. Army Corps of Engineers

1028 - U.S. Geological Survey

46001 - South Dakota State University Soils Laboratory

46002 - South Dakota Water Resources Institute

46003 - South Dakota Agricultural Experiment Station

46004 - South Dakota State Chemist

46006 - South Dakota State University, Department Station Biochemistry

46007 - South Dakota Department of Environment and Natural Resources, Division of Water Rights

46008 - South Dakota Department of Environment and Natural Resources, Division of Geological Survey

80020 - National Water-Quality Laboratory, Denver, Colorado

83113 - District Water-Quality Laboratory, Lincoln, Nebraska

84610 - National Uranium Resources Evaluation

84913 - U.S. Geological Survey Water Quality Laboratory, Salt Lake City, Utah

9746 - South Dakota--specific entity unknown

99001 - Private contractor

The number under each parameter in the header is used when retrieving data from WATSTORE.

Chemical analyses are listed by aquifer in the following order:

$\begin{array}{ll}\text { Choteau } & \text { Pleistocene } \\ \text { Corsica } & \text { Till } \\ \text { Delmont } & \text { Codell } \\ \text { Geddes } & \text { Dakota } \\ \text { Greenwood } & \text { Niobrara } \\ \text { lntermediate } & \text { Pierre Shale }\end{array}$


Table 3. Chemical analyses of ground water in Charles Mix and Douglas Counties

\begin{tabular}{|c|c|c|c|c|c|c|c|c|c|c|}
\hline $\begin{array}{l}\text { Principal aquifer/ } \\
\text { local Identifier }\end{array}$ & $\begin{array}{l}\text { Date of } \\
\text { sampla }\end{array}$ & $\begin{array}{c}\text { Depth } \\
\text { of } \\
\text { well } \\
\text { (feet) } \\
\text { (00003) }\end{array}$ & $\begin{array}{c}\text { Agency } \\
\text { collecting } \\
\text { sampla } \\
\text { (code } \\
\text { number) } \\
\text { (00027) }\end{array}$ & $\begin{array}{c}\text { Agency } \\
\text { analyzing } \\
\text { aampla } \\
\text { (code } \\
\text { number) } \\
\text { (00028) }\end{array}$ & $\begin{array}{l}\text { Water } \\
\text { tamper- } \\
\text { ature } \\
\left({ }^{\circ} \mathrm{C}\right) \\
(00010)\end{array}$ & $\begin{array}{c}\text { Specific } \\
\text { conduct- } \\
\text { ance } \\
(\mu \mathrm{S} / \mathrm{cm}) \\
(00095)\end{array}$ & $\begin{array}{c}\text { pH } \\
\text { (standard } \\
\text { units) } \\
(00400)\end{array}$ & $\begin{array}{c}\text { Hardness } \\
\text { total } \\
\left(\mathrm{mgll}^{2} \text { as }\right. \\
\left.\mathrm{CaCO}_{3}\right) \\
(00900)\end{array}$ & $\begin{array}{c}\text { Calcium, } \\
\text { dissolved } \\
\text { (mg/L } \\
\text { aa Ca) } \\
(00915)\end{array}$ & $\begin{array}{l}\text { Magne- } \\
\text { aium, } \\
\text { diasolved } \\
\text { (mg/l } \\
\text { aa } \mathrm{Mg}) \\
\text { (00925) }\end{array}$ \\
\hline
\end{tabular}

Choteau aquifer

\begin{tabular}{|c|c|c|c|c|c|c|c|c|c|c|}
\hline 94N61W19BBBD & $11-08-76$ & 30 & 1028 & 1028 & -- & 995 & 7.3 & 450 & 130 & 30 \\
\hline 94N61W19CCCC & $10-26-66$ & 29 & 1028 & 46002 & 11.0 & 1,480 & 7.4 & 720 & 250 & 24 \\
\hline 95N62W 8ADDD & $09-18-67$ & 236 & 1028 & 1028 & 11.0 & 3,210 & 7.7 & 1,500 & 450 & 90 \\
\hline --do-- & $05-\quad-69$ & 236 & 1028 & 1028 & -- & 2,990 & 7.2 & 1,300 & 380 & 78 \\
\hline --do-- & $03-03-75$ & 236 & 1028 & 1028 & -- & 1,640 & 7.1 & 960 & 270 & 70 \\
\hline --do-- & $08-24-79$ & 236 & 1028 & 1028 & 21.0 & 2,650 & -- & 1,500 & 440 & 100 \\
\hline 95N62W18CAAC & $02-13-76$ & 200 & 1028 & 1028 & - & 2,190 & 7.4 & 470 & 130 & 36 \\
\hline 95N62W33B & $-\quad-68$ & 170 & 1028 & 1028 & -- & 2,510 & 7.7 & 1,100 & 320 & 67 \\
\hline 95N62W33BBBB & $08-24-67$ & 168 & 1028 & 46002 & 13.0 & 2,810 & 7.9 & 880 & 250 & 62 \\
\hline --do-- & $09-19-68$ & 168 & 1028 & 1028 & -- & 2,870 & 8.0 & 1,100 & 330 & 74 \\
\hline- do-- & $10-02-68$ & 168 & 1028 & 1028 & -- & 2,510 & 7.7 & 1,100 & 320 & 67 \\
\hline- do- & $07-10-73$ & 168 & 1028 & 1028 & -- & 2,460 & 6.3 & 750 & 250 & 29 \\
\hline --do-- & $08-24-79$ & 168 & 1028 & 1028 & 21.0 & 1,860 & - & 550 & 160 & 36 \\
\hline 95N63W 4ABDA & $11-10-54$ & 231 & 46004 & 46004 & -- & -- & 7.6 & 390 & 110 & 27 \\
\hline --do-- & $04-26-66$ & 231 & 1028 & 1028 & - & -- & 7.8 & 160 & 23 & 26 \\
\hline-- do-- & $08-30-72$ & 231 & 1028 & 1028 & - & 2,270 & 8.2 & 390 & 120 & 23 \\
\hline 95N63W 4DABB & $01-17-70$ & 243 & 1028 & 99001 & -- & -- & 7.4 & 480 & 110 & 49 \\
\hline --do-- & $08-30-72$ & 243 & 1028 & 1028 & - & -- & 8.1 & 420 & 130 & 23 \\
\hline --do-- & $01-13-76$ & 243 & 1028 & 1028 & -- & 2,470 & 7.8 & 420 & 120 & 29 \\
\hline 96N62W18AAC & $09-02-76$ & 300 & 1028 & 1028 & - & 2,380 & 7.1 & 1,200 & 400 & 52 \\
\hline $96 \mathrm{~N} 63 \mathrm{~W} 3 \mathrm{DCDA}$ & $04-08-81$ & 180 & 1028 & 1028 & -- & 2,700 & 8.5 & 1,500 & 260 & 200 \\
\hline 96N63W 8BDBB & $01-27-75$ & 240 & 1028 & 1028 & -- & 2,070 & 7.4 & 400 & 120 & 24 \\
\hline --do-- & $04-11-80$ & 240 & 1028 & 1028 & - & 1,820 & 7.5 & 270 & 78 & 18 \\
\hline 96N63W10DDB & --76 & 200 & 1028 & 1028 & -- & 234 & 7.0 & 490 & 130 & 38 \\
\hline --do-- & $04-08-77$ & 200 & 1028 & 1028 & - & 1,640 & 7.5 & 410 & 120 & 26 \\
\hline 96N63W14BBBB & $11-21-72$ & 210 & 1028 & 1028 & -- & 2,550 & 7.6 & 740 & 240 & 36 \\
\hline --do-- & $08-21-79$ & 210 & 1028 & 1028 & 21.0 & 2,450 & $\cdots$ & 670 & 200 & 45 \\
\hline $96 \mathrm{~N} 63 \mathrm{~W} 16 \mathrm{CCCC}$ & $06-27-67$ & 200 & 46002 & 46002 & 16.0 & 1,980 & 8.4 & 320 & 120 & 3.8 \\
\hline --do-- & $08-24-67$ & 200 & 1028 & 1028 & -- & 1,980 & 8.4 & 310 & 120 & 3.8 \\
\hline --do-- & $05-\quad-69$ & 200 & 1028 & 1028 & -- & 1,960 & 7.8 & 310 & 88 & 23 \\
\hline --do-- & $06-13-74$ & 200 & 1028 & 1028 & -- & 2,060 & 7.7 & 340 & 100 & 23 \\
\hline $96 \mathrm{~N} 63 \mathrm{~W} 16 \mathrm{D}$ & $09-09-75$ & 181 & 1028 & 1028 & -- & 1,970 & 8.2 & 340 & 91 & 27 \\
\hline 96N63W16DDB & $-\quad-76$ & 255 & 1028 & 1028 & $\cdots$ & 2,110 & 7.2 & 340 & 120 & 12 \\
\hline 96N63W17D & $04-21-75$ & 155 & 1028 & 1028 & -- & 1,880 & 7.1 & 480 & 130 & 37 \\
\hline 96N63W17DDA & $04-21-75$ & 155 & 1028 & 1028 & -- & 1,880 & 7.1 & 440 & 130 & 27 \\
\hline
\end{tabular}




\begin{tabular}{|c|c|c|c|c|c|c|c|c|c|c|c|c|}
\hline $\begin{array}{l}\text { Sodium, } \\
\text { dis- } \\
\text { solved } \\
\text { (mg/L } \\
\text { as } \mathrm{Na} \text { ) } \\
(00930)\end{array}$ & $\begin{array}{l}\text { Potas- } \\
\text { sium, } \\
\text { dis- } \\
\text { solved } \\
\text { (mg/L } \\
\text { as K) } \\
\text { (00935) }\end{array}$ & $\begin{array}{c}\text { Chloride, } \\
\text { dis- } \\
\text { solved } \\
\text { (mg/L } \\
\text { as Cl) } \\
(00940)\end{array}$ & $\begin{array}{c}\text { Sulfate, } \\
\text { dis- } \\
\text { solved } \\
\text { (mg/L } \\
\left.\text { as } \mathrm{SO}_{4}\right) \\
(00945)\end{array}$ & $\begin{array}{c}\text { Fluoride, } \\
\text { dis- } \\
\text { solved } \\
\text { (mg/L } \\
\text { as F) } \\
(00950)\end{array}$ & $\begin{array}{c}\text { Silica, } \\
\text { dis- } \\
\text { solved } \\
\left(\mathrm{mg}^{2} \mathrm{~L} \text { as }\right. \\
\text { SiO }) \\
(00955)\end{array}$ & $\begin{array}{c}\text { Bicar- } \\
\text { bonate } \\
\text { FET- } \\
\text { FLD } \\
\text { (mgllas } \\
\left.\mathrm{HCO}_{3}\right) \\
(00440)\end{array}$ & $\begin{array}{c}\text { Carbo- } \\
\text { nate } \\
\text { FET- } \\
\text { FLD } \\
(\mathrm{mg} / \mathrm{l} \text { as } \\
\left.\mathrm{CO}_{3}\right) \\
(00445)\end{array}$ & $\begin{array}{c}\text { Nitro- } \\
\text { gen, } \\
\text { nitrate } \\
\text { total } \\
\text { (mg/L } \\
\text { as N) } \\
(00620)\end{array}$ & $\begin{array}{l}\text { Solids, } \\
\text { sum of } \\
\text { consti- } \\
\text { tuents, } \\
\text { dis- } \\
\text { solved } \\
\text { (mg/L) } \\
\text { (70301) }\end{array}$ & $\begin{array}{l}\text { Iron, } \\
\text { total } \\
\text { recover- } \\
\text { able } \\
(\mu \mathrm{g} / \mathrm{L} \\
\text { as Fe) } \\
(01045)\end{array}$ & $\begin{array}{c}\text { Manga- } \\
\text { nese, } \\
\text { total } \\
\text { recover- } \\
\text { able } \\
(\mu g / l \\
\text { as Mn) } \\
(01055)\end{array}$ & $\begin{array}{c}\text { Boron, } \\
\text { dis- } \\
\text { solved } \\
(\mu g / L \\
\text { as B) } \\
(01020)\end{array}$ \\
\hline
\end{tabular}

\begin{tabular}{|c|c|c|c|c|c|c|c|c|c|c|c|c|}
\hline 27 & 9.0 & 12 & 420 & - & -. & 160 & -- & -- & 710 & -- & -- & -- \\
\hline 39 & 9.4 & 10 & 500 & -- & - & 280 & 0 & -- & 968 & -- & -- & -- \\
\hline 250 & 42 & 16 & 1,500 & .. & -. & 320 & -- & -- & 2,560 & -- & .. & -- \\
\hline 280 & 33 & 15 & 1,900 & .- & -. & -- & -- & 0.140 & 2,720 & 2,300 & -- & 1,400 \\
\hline 160 & 28 & 37 & 1,000 & -. & - & 63 & -- & 1.70 & 1,600 & 80 & -- & 970 \\
\hline 260 & -- & 15 & 1,800 & 0.41 & -- & -- & -- & -- & 3,000 & 2,200 & 4,100 & - \\
\hline 420 & 30 & 150 & 850 & -- & - & 180 & -- & -- & 1,700 & -. & -- & -- \\
\hline 320 & 33 & 36 & -- & -- & -- & -- & -- & 3.00 & -- & -- & -- & -- \\
\hline 300 & 25 & 68 & 1,100 & -- & - & -- & -- & .150 & 1,850 & 690 & -- & -- \\
\hline 300 & 30 & 36 & 1,500 & .40 & 17 & 260 & ND & . 100 & 2,460 & 780 & 1,400 & 1,800 \\
\hline 320 & 33 & 36 & 1,700 & -. & - & -- & -. & 2.60 & 2,550 & ND & -- & -- \\
\hline 290 & 30 & 83 & 1,200 & -. & - & 300 & -- & .280 & 2,030 & 190 & -- & 1,200 \\
\hline 310 & -- & 120 & 850 & .49 & -- & -- & -- & -- & -- & 80 & 2,300 & -- \\
\hline -- & -- & 150 & 920 & .80 & - & 220 & -- & .00 & -- & 120 & -- & -- \\
\hline 400 & 22 & 130 & 920 & .70 & - & 200 & -. & -- & 1,620 & -- & -- & -- \\
\hline 390 & 20 & 150 & 930 & -- & -- & 190 & .- & .00 & 1,720 & 1,800 & 1,700 & -- \\
\hline -. & -- & 140 & 600 & .0 & -- & 270 & 0 & -- & -- & -- & -. & -- \\
\hline 380 & 20 & 140 & 920 & 1.1 & - & 210 & -- & 1.00 & 1,720 & 500 & 2,000 & -- \\
\hline 390 & 23 & 140 & 930 & .60 & - & 200 & .- & 1.00 & 1,720 & 540 & 2,000 & -- \\
\hline 130 & 39 & 10 & 1,200 & -- & -- & 410 & -- & -- & 2,010 & -- & -- & -- \\
\hline 370 & 31 & 120 & 1,900 & -- & -- & 180 & 29 & -- & 3,020 & -- & -- & -- \\
\hline 360 & 25 & 170 & 610 & -. & - & 250 & -- & -. & 1,440 & - & -- & -- \\
\hline 320 & 16 & 220 & 420 & -- & -. & 310 & -- & -- & 1,230 & -- & -- & -- \\
\hline 370 & 28 & 47 & -. & -- & -- & -- & - & -- & -- & -- & -- & - \\
\hline 230 & 26 & 45 & 530 & -- & -- & 330 & -- & -- & 1,140 & -- & -- & -- \\
\hline 260 & 28 & 25 & 1,000 & -- & -- & -- & -. & .100 & 1,730 & 110 & -- & 690 \\
\hline 330 & -- & 100 & 820 & .40 & - & -- & -- & -- & -- & 1500 & 1,200 & -- \\
\hline 320 & 20 & 220 & 410 & -. & -- & 240 & -- & -. & 1,210 & -- & -. & -- \\
\hline 320 & 20 & 220 & 410 & -. & -- & -- & -- & 1.20 & 1,220 & 2,900 & -- & -- \\
\hline 330 & 21 & 200 & 560 & -- & -- & -- & -- & .310 & 1,370 & 2,000 & -. & 1,800 \\
\hline 360 & 28 & 220 & 490 & -- & -- & 280 & -- & .100 & 1,360 & 60 & -- & 2,100 \\
\hline 390 & 6.0 & 140 & 500 & -- & - & 350 & -- & - & 1,320 & -- & -- & 2,700 \\
\hline 320 & 33 & 130 & -- & -- & -- & -- & -- & -- & -- & -- & - & -- \\
\hline 360 & 29 & 150 & 580 & -- & -- & 280 & -- & -- & 1,420 & -- & -- & - \\
\hline 360 & 29 & 150 & 580 & -- & .. & 280 & -- & -- & 1,410 & -- & -- & - \\
\hline
\end{tabular}


Table 3. Chemical analyses of ground water in Charles Mix and Douglas Counties--Continued

\begin{tabular}{|c|c|c|c|c|c|c|c|c|c|c|}
\hline $\begin{array}{l}\text { Principal aquifer/ } \\
\text { local Identifier }\end{array}$ & $\begin{array}{l}\text { Date of } \\
\text { sample }\end{array}$ & $\begin{array}{l}\text { Depth } \\
\text { of } \\
\text { well } \\
\text { (feet) } \\
(00003)\end{array}$ & $\begin{array}{c}\text { Agency } \\
\text { collecting } \\
\text { sample } \\
\text { (code } \\
\text { number) } \\
\text { (00027) }\end{array}$ & $\begin{array}{c}\text { Agency } \\
\text { analyzing } \\
\text { sample } \\
\text { (code } \\
\text { number) } \\
\text { (00028) }\end{array}$ & $\begin{array}{c}\text { Water } \\
\text { temper- } \\
\text { ature } \\
\left({ }^{\circ} \mathrm{C}\right) \\
(00010)\end{array}$ & $\begin{array}{c}\text { Specific } \\
\text { conduct- } \\
\text { ance } \\
(\mu \mathrm{S} / \mathrm{cm}) \\
(00095)\end{array}$ & $\begin{array}{c}\text { pH } \\
\text { (standard } \\
\text { unlts) } \\
(00400)\end{array}$ & $\begin{array}{c}\text { Hardness } \\
\text { total } \\
\text { (mg/L as } \\
\left.\mathrm{CeCO}_{3}\right) \\
(00900)\end{array}$ & $\begin{array}{l}\text { Calcium, } \\
\text { dissolved } \\
\text { (mg/L } \\
\text { as Ca) } \\
\text { (00915) }\end{array}$ & $\begin{array}{l}\text { Magne- } \\
\text { sium, } \\
\text { dissolved } \\
\text { (mg/L } \\
\text { as } \mathrm{Mg}) \\
(00925)\end{array}$ \\
\hline
\end{tabular}

Choteau aquifer (Cont.)

\begin{tabular}{|c|c|c|c|c|c|c|c|c|c|c|}
\hline 96N63W19ABCC & $03-21-77$ & 220 & 1028 & 1028 & -. & 699 & 7.2 & 94 & 26 & 7.0 \\
\hline --do-- & $06-03-77$ & 220 & 1028 & 1028 & - & 1,970 & 7.2 & 320 & 100 & 14 \\
\hline 96N63W20AAAB & $05-15-80$ & 183 & 1028 & 1028 & -. & 1,800 & 7.9 & 260 & 60 & 26 \\
\hline 96N63W22BBD & $06-14-76$ & 200 & 1028 & 1028 & -- & 1,610 & 7.3 & 520 & 150 & 35 \\
\hline $96 \mathrm{~N} 63 \mathrm{~W} 22 \mathrm{CCA}$ & $06-14-76$ & 200 & 1028 & 1028 & $-\cdot$ & 1,610 & 7.3 & 520 & 150 & 35 \\
\hline $96 \mathrm{~N} 63 \mathrm{~W} 24 \mathrm{BBBC}$ & $07-10-73$ & 231 & 1028 & 1028 & -- & 2,150 & 7.7 & 670 & 220 & 31 \\
\hline --do-- & $08-21-79$ & 231 & 1028 & 1028 & 21.0 & 2,850 & -. & 930 & 260 & 72 \\
\hline $96 \mathrm{~N} 63 \mathrm{~W} 26 \mathrm{C}$ & $04-21-75$ & 175 & 1028 & 1028 & -- & 2,030 & 7.1 & 440 & 130 & 29 \\
\hline 96N63W26DDDD & $08-01-68$ & 188 & 1028 & 46002 & 12.0 & 1,710 & 7.8 & 320 & 100 & 18 \\
\hline --do-- & $05-\quad-69$ & 188 & 1028 & 1028 & -- & 1,980 & 7.7 & 410 & 120 & 30 \\
\hline --do-- & $06-13-74$ & 188 & 1028 & 1028 & .. & 1,910 & 7.5 & 400 & 110 & 30 \\
\hline --do-- & $08-21-79$ & 188 & 1028 & 1028 & 25.0 & 1,600 & -- & 320 & 88 & 24 \\
\hline 96N63W34DCDD & $12-17-57$ & 158 & 46004 & 46004 & -- & -- & 8.3 & 340 & 100 & 20 \\
\hline $96 \mathrm{~N} 63 \mathrm{~W} 35 \mathrm{BCCC}$ & $06-30-75$ & 220 & 1028 & 1028 & -- & 1,630 & 7.3 & 780 & 200 & 67 \\
\hline 96N64W 6D & $07-09-75$ & 204 & 1028 & 1028 & -- & 2,390 & 7.8 & 330 & 90 & 26 \\
\hline $96 \mathrm{~N} 64 \mathrm{~W} 8 \mathrm{CBBB}$ & $00-00-65$ & 283 & 46008 & 46008 & -- & -. & 7.3 & 500 & 140 & 34 \\
\hline 96N64W12ACCA & $-\quad-68$ & 290 & 1028 & 1028 & -- & 2,250 & -- & 360 & 120 & 17 \\
\hline --do-- & $05-04-76$ & 290 & 1028 & 1028 & -- & 2,170 & 8.1 & 400 & 120 & 24 \\
\hline $96 \mathrm{~N} 64 \mathrm{~W} 12 \mathrm{CBBB}$ & $08-01-68$ & 233 & 1028 & 46002 & 12.0 & 2,250 & 8.0 & 370 & 120 & 17 \\
\hline --do-- & $10-17.69$ & 233 & 1028 & 1028 & -. & 2,410 & 7.5 & 500 & 150 & 30 \\
\hline $96 \mathrm{~N} 64 \mathrm{~W} 12 \mathrm{CBBB}$ & $08-21-79$ & 233 & 1028 & 1028 & 25.0 & 2,200 & -- & 340 & 100 & 23 \\
\hline 96N64W18ADAB & --65 & 200 & 46008 & 46008 & -- & -- & 7.5 & 180 & 59 & 8.0 \\
\hline 96N64W19AAAA & --65 & 248 & 46008 & 46008 & -- & -- & 8.2 & 340 & 80 & 34 \\
\hline 96N64W20AAAA & $-\quad-65$ & 242 & 46008 & 46008 & -- & -- & 7.3 & 530 & 150 & 38 \\
\hline 96N65W 1ABCB & $08-06-64$ & 176 & 1028 & 46001 & 14.5 & 2,450 & 7.6 & 460 & 130 & 34 \\
\hline --do-- & $07-14-67$ & 176 & 1028 & 46002 & -- & 2,810 & 7.4 & 840 & 250 & 53 \\
\hline --do-- & $08-01-67$ & 176 & 1028 & 46002 & -- & 2,340 & 7.4 & 540 & 160 & 33 \\
\hline --do-- & $08-16-67$ & 176 & 1028 & 46002 & 14.0 & 2,740 & 7.4 & 540 & 180 & 22 \\
\hline $96 \mathrm{~N} 65 \mathrm{~W} 1 \mathrm{BBBB}$ & $06-\quad-65$ & 175 & 46008 & 46008 & -- & -- & 7.2 & 520 & 150 & 34 \\
\hline 96N65W 3AABB & $06-\quad-65$ & 140 & 46008 & 46008 & -. & -- & 7.4 & 580 & 180 & 29 \\
\hline $96 \mathrm{~N} 65 \mathrm{~W} 4 \mathrm{CDCA}$ & $10--53$ & 200 & 1028 & 1028 & -- & $\cdots$ & 7.5 & 670 & 190 & 45 \\
\hline --do-- & $02-13-57$ & 200 & 46004 & 46004 & -- & -. & 7.6 & 760 & 210 & 56 \\
\hline --do-- & $09-\quad-65$ & 200 & 1028 & 1028 & -- & -. & 6.9 & 730 & 220 & 46 \\
\hline --do-- & $01-11-71$ & 200 & 1028 & 1028 & - & -- & 7.2 & 750 & 230 & 43 \\
\hline 96N65W 5DADD & $10-17-69$ & 147 & 1028 & 1028 & -- & 1,700 & 7.6 & 470 & 140 & 29 \\
\hline
\end{tabular}




\begin{tabular}{|c|c|c|c|c|c|c|c|c|c|c|c|c|}
\hline $\begin{array}{c}\text { Sodium, } \\
\text { dis- } \\
\text { solved } \\
\text { (mg/L } \\
\text { as } \mathrm{Na} \text { ) } \\
(00930)\end{array}$ & $\begin{array}{l}\text { Potas- } \\
\text { sium, } \\
\text { dis- } \\
\text { solved } \\
\text { (mg/L } \\
\text { ss K) } \\
(00935)\end{array}$ & $\begin{array}{c}\text { Chloride, } \\
\text { dis- } \\
\text { solved } \\
\text { (mg/l } \\
\text { as Cl) } \\
(00940)\end{array}$ & $\begin{array}{c}\text { Sulfate, } \\
\text { dis- } \\
\text { solved } \\
\text { (mg/L } \\
\left.\text { as } \mathrm{SO}_{4}\right) \\
(00945)\end{array}$ & $\begin{array}{c}\text { Fluoride, } \\
\text { dis- } \\
\text { solved } \\
\text { (mg/L } \\
\text { as F) } \\
(00950)\end{array}$ & $\begin{array}{c}\text { Silica, } \\
\text { dis- } \\
\text { solved } \\
(\mathrm{mg} / \mathrm{L} \text { as } \\
\left.\mathrm{SiO}_{2}\right) \\
(00955)\end{array}$ & $\begin{array}{c}\text { Bicar- } \\
\text { bonate } \\
\text { FET- } \\
\text { FLD } \\
\left(\mathrm{mg}^{2} \text { as }\right. \\
\left.\mathrm{HCO}_{3}\right) \\
(00440)\end{array}$ & $\begin{array}{c}\text { Carbo- } \\
\text { nate } \\
\text { FET- } \\
\text { FLD } \\
(\mathrm{mg} / \mathrm{L} \text { as } \\
\mathrm{CO} \\
(00445)\end{array}$ & $\begin{array}{c}\text { Nitro- } \\
\text { gen, } \\
\text { nitrate } \\
\text { total } \\
\text { (mg/l } \\
\text { as N) } \\
(00620)\end{array}$ & $\begin{array}{c}\text { Solids, } \\
\text { sum of } \\
\text { consti- } \\
\text { tuents, } \\
\text { dis- } \\
\text { solved } \\
\text { (mg/L) } \\
\text { (70301) }\end{array}$ & $\begin{array}{c}\text { Iron, } \\
\text { total } \\
\text { recover- } \\
\text { able } \\
(\mu \mathrm{g} / \mathrm{L} \\
\text { as Fe) } \\
(01045)\end{array}$ & $\begin{array}{c}\text { Manga- } \\
\text { nese, } \\
\text { total } \\
\text { recover- } \\
\text { able } \\
(\mu g / L \\
\text { as Mn) } \\
(01055)\end{array}$ & $\begin{array}{c}\text { Boron, } \\
\text { dis- } \\
\text { solved } \\
(\mu g / L \\
\text { as B) } \\
(01020)\end{array}$ \\
\hline
\end{tabular}

\begin{tabular}{|c|c|c|c|c|c|c|c|c|c|c|c|c|}
\hline 120 & 6.0 & 79 & 200 & -- & -- & 120 & - & -- & 497 & -- & - & -- \\
\hline 290 & 23 & 210 & 410 & $\cdots$ & -- & 300 & -- & -- & 1,200 & -- & - & -- \\
\hline 300 & 26 & 250 & 480 & -- & -- & 210 & -- & 1.60 & 1,240 & 310 & -- & 1,700 \\
\hline 270 & 28 & 120 & 450 & $\cdots$ & -- & 530 & -- & -- & 1,310 & -- & -- & - \\
\hline 270 & 28 & 120 & 450 & -- & -- & 530 & -- & -- & 1,310 & -- & -- & -- \\
\hline 210 & 28 & 36 & 960 & -- & -- & 460 & - & .110 & 1,710 & 60 & -- & 750 \\
\hline 360 & - & 110 & 1,100 & 0.42 & -- & -- & -- & -- & -- & 700 & 1,900 & -- \\
\hline 360 & 28 & 150 & 610 & -- & -- & 270 & -- & -- & 1,440 & -- & -- & -- \\
\hline 260 & 19 & 130 & 500 & - & -- & 310 & - & -- & 1,180 & -- & -- & -- \\
\hline 280 & 22 & 120 & 600 & - & -- & -- & - & ND & 1,330 & 280 & -- & 950 \\
\hline 330 & 24 & 140 & 480 & -- & -- & 360 & - & .010 & 1,290 & 80 & - & 800 \\
\hline 270 & -- & 150 & 330 & .40 & -- & -- & -- & -- & -- & $<50$ & 1,700 & $\cdots$ \\
\hline 410 & 19 & 250 & 600 & .60 & -- & 260 & -- & .800 & 1,530 & 200 & 1,600 & -- \\
\hline 120 & 10 & 39 & 620 & -- & -- & 290 & - & -- & 1,200 & -- & -- & -- \\
\hline 510 & 32 & 250 & 560 & -- & -- & 350 & -- & -- & 1,640 & - & -- & -- \\
\hline -- & -- & 250 & 940 & -- & -- & -- & -- & -- & 1,970 & -- & -- & -- \\
\hline 400 & 24 & 220 & -- & -- & -- & -- & -- & -- & - & 80 & -- & -- \\
\hline 330 & 27 & 210 & 510 & -- & -- & 290 & 12 & - & 1,380 & -- & -- & -- \\
\hline 400 & 26 & 220 & 690 & -. & -- & 280 & -- & -. & 1,610 & -- & -- & -- \\
\hline 390 & 27 & 190 & 810 & -- & -- & -- & -- & ND & 1,740 & 10 & -- & 3,500 \\
\hline 400 & - & 200 & 580 & .41 & -- & -- & -- & -. & -- & 70 & 1,500 & - \\
\hline-- & -. & 78 & 72 & -- & -- & -- & -- & -- & 810 & -- & -- & -- \\
\hline -- & -- & 130 & 210 & -- & -- & -- & -- & -- & - & -- & $-\cdots$ & -- \\
\hline -- & - & 140 & 840 & -- & -- & -- & -- & -- & 1,870 & -- & -- & -- \\
\hline 460 & 27 & 180 & 1,100 & -- & -- & 330 & 0 & $\cdots$ & 2,090 & -- & -- & -- \\
\hline 440 & 29 & 140 & 1,000 & -- & -- & 350 & -- & -- & 2,080 & -- & -- & -- \\
\hline 410 & 25 & 140 & 800 & -- & -- & 300 & -. & -- & 1,720 & -- & -- & -- \\
\hline 290 & 40 & 160 & 780 & -. & -- & 300 & -- & - & 1,620 & -- & -- & 1,800 \\
\hline -- & -- & 160 & 940 & -- & - & -- & -- & - & -- & 60 & 1,400 & -- \\
\hline -- & -- & 120 & 980 & -- & -- & -- & -- & - & -- & 800 & $-\cdot$ & -- \\
\hline -- & -- & 17 & 620 & .65 & -- & 330 & -- & .00 & -- & 900 & -- & -- \\
\hline 140 & 26 & 27 & 730 & .60 & -- & 360 & -- & .00 & 1,370 & 700 & 2,800 & -- \\
\hline 180 & 26 & 52 & 810 & .60 & -- & 320 & $-\infty$ & 3.00 & 1,480 & 400 & 3,200 & -- \\
\hline 180 & 73 & 58 & 830 & .57 & -- & 310 & -- & .00 & 1,560 & 400 & 2,700 & -- \\
\hline 210 & 27 & 20 & 750 & -- & -- & -- & -- & ND & 1,280 & ND & -- & 1,400 \\
\hline
\end{tabular}


Table 3. Chemical analyses of ground water in Charles Mix and Douglas Counties--Continued

\begin{tabular}{|c|c|c|c|c|c|c|c|c|c|c|}
\hline $\begin{array}{l}\text { Principal aquifer/ } \\
\text { local Identifier }\end{array}$ & $\begin{array}{l}\text { Date of } \\
\text { aample }\end{array}$ & $\begin{array}{c}\text { Depth } \\
\text { of } \\
\text { well } \\
\text { (feet) } \\
(00003)\end{array}$ & $\begin{array}{c}\text { Agency } \\
\text { collecting } \\
\text { aample } \\
\text { (code } \\
\text { number) } \\
\text { (00027) }\end{array}$ & $\begin{array}{c}\text { Agency } \\
\text { analyzing } \\
\text { aample } \\
\text { (code } \\
\text { number) } \\
\text { (00028) }\end{array}$ & $\begin{array}{l}\text { Water } \\
\text { temper- } \\
\text { ature } \\
\left({ }^{\circ} \mathrm{C}\right) \\
(00010)\end{array}$ & $\begin{array}{c}\text { Specific } \\
\text { conduct- } \\
\text { ance } \\
(\mu S / \mathrm{cm}) \\
(00095)\end{array}$ & $\begin{array}{c}\text { pH } \\
\text { (atandard } \\
\text { units) } \\
(00400)\end{array}$ & $\begin{array}{c}\text { Herdnesa } \\
\text { total } \\
(\mathrm{mghles} \\
\left.\mathrm{CaCO}_{3}\right) \\
(00900)\end{array}$ & $\begin{array}{c}\text { Calcium, } \\
\text { dissolved } \\
\text { (mg/L } \\
\text { aa Ca) } \\
(00915)\end{array}$ & $\begin{array}{l}\text { Megne- } \\
\text { sium, } \\
\text { dissolved } \\
\text { (mg/L } \\
\text { as Mg) } \\
(00925)\end{array}$ \\
\hline
\end{tabular}

Choteau aquifer (Cont.)

\begin{tabular}{|c|c|c|c|c|c|c|c|c|c|c|}
\hline & & & & & & & & & & \\
\hline 96N65W 5DADD & $08-20-79$ & 147 & 1028 & 1028 & 25.0 & 1,600 & - & 430 & 130 & 26 \\
\hline 96N65W 5DCC & $09-29-56$ & 164 & 1028 & 46001 & -- & 1,400 & 8.2 & 620 & 120 & 78 \\
\hline --do-- & $-\quad-65$ & 164 & 46008 & 46008 & -- & -- & 7.5 & 370 & 110 & 22 \\
\hline 96N65W 8AAAC & $-\quad-68$ & 140 & 1028 & 1028 & - & 1,270 & 7.8 & 490 & 140 & 31 \\
\hline $96 \mathrm{~N} 65 \mathrm{~W} 8 \mathrm{AABB}$ & $01-29-81$ & 96 & 1028 & 1028 & -- & 1,190 & 7.3 & 630 & 170 & 48 \\
\hline 96N65W 9ADBA & $10-03-69$ & 123 & 1028 & 1028 & 12.0 & 2,360 & 7.7 & 1,200 & 250 & 150 \\
\hline --do-- & $10-09-69$ & 123 & 1028 & 46002 & - & 2,360 & 7.7 & 1,200 & 250 & 150 \\
\hline --do-- & $10-17-69$ & 123 & 1028 & 1028 & -- & 2,460 & 7.6 & 1,100 & 300 & 73 \\
\hline --do-- & $06-13-74$ & 123 & 1028 & 1028 & -- & 2,920 & 7.6 & 1,300 & 390 & 81 \\
\hline --do-- & $08-23-79$ & 123 & 1028 & 1028 & -- & 2,720 & -- & 950 & 250 & 78 \\
\hline $96 \mathrm{~N} 65 \mathrm{~W} 12 \mathrm{ACAA}$ & $08-25-76$ & 200 & 1028 & 1028 & -- & 1,710 & 7.0 & 920 & 280 & 56 \\
\hline 97N62W30DDB & $-\quad-76$ & 180 & 1028 & 1028 & -- & 1,350 & 7.4 & 690 & 190 & 54 \\
\hline --do-- & $08-19-76$ & 180 & 1028 & 1028 & -- & 2,500 & 7.4 & 690 & 210 & 40 \\
\hline 97N62W31DDDD & $08-21-79$ & 225 & 1028 & 1028 & 21.0 & 3,350 & -- & -- & -- & -- \\
\hline 97N63W 2DDDD & $11-21-72$ & 168 & 1028 & 1028 & -- & 2,060 & 7.3 & 960 & 300 & 51 \\
\hline --do-- & $08-23-79$ & 168 & 1028 & 1028 & 21.0 & 1,950 & -- & 850 & 230 & 71 \\
\hline 97N63W 4DACB & $02-11-77$ & 190 & 1028 & 1028 & -- & 1,640 & 7.4 & 380 & 110 & 27 \\
\hline 97N63W11D2 & $02-18-82$ & 170 & 1028 & 1028 & -- & 2,100 & 7.6 & 1,200 & 370 & 72 \\
\hline 97N63W17DBCC & $-\quad-76$ & 210 & 1028 & 1028 & -- & 1,950 & 7.1 & 200 & 60 & 13 \\
\hline --do-- & $04-07-81$ & 210 & 1028 & 1028 & -- & 1,550 & 8.1 & 300 & 68 & 31 \\
\hline 97N63W20C & $02-16-82$ & 210 & 1028 & 1028 & -- & 1,800 & 8.0 & 260 & 64 & 24 \\
\hline 97N63W26AAAA & $05-15-80$ & 202 & 1028 & 1028 & -. & 1,850 & 6.9 & 180 & 40 & 19 \\
\hline 97N63W28DDDD & $06-21-67$ & 166 & 1028 & 46002 & 16.0 & 2,410 & 6.6 & 820 & 220 & 65 \\
\hline --do-- & $05-\quad-69$ & 166 & 1028 & 1028 & - & 1,710 & 6.9 & 500 & 140 & 37 \\
\hline --do-- & $06-13-74$ & 166 & 1028 & 1028 & - & 1,980 & 6.2 & 510 & 150 & 32 \\
\hline- -do-- & $08-24-79$ & 166 & 1028 & 1028 & 21.0 & 1,600 & -- & 490 & 140 & 33 \\
\hline 97N63W30BDBB & $-\quad-75$ & 175 & 1028 & 1028 & -- & 1,890 & 8.0 & 84 & 22 & 7.0 \\
\hline 97N63W32AAC & $12-11-80$ & 190 & 1028 & 1028 & -- & 1,840 & 7.2 & 210 & 56 & 17 \\
\hline --do-- & $01-19-81$ & 190 & 1028 & 1028 & -- & 1,920 & 7.2 & 400 & 120 & 26 \\
\hline --do-- & $04-16-81$ & 190 & 1028 & 1028 & - & 1,700 & 7.6 & 400 & 110 & 29 \\
\hline 97N63W33AAAA & $05-15-80$ & 183 & 1028 & 1028 & -- & 2,150 & 7.8 & 740 & 200 & 58 \\
\hline 97N64W10BACC & $-\quad-68$ & 200 & 1028 & 1028 & -- & 1,890 & 7.5 & 200 & 62 & 12 \\
\hline --do-- & $-\quad-75$ & 200 & 1028 & 1028 & - & 1,940 & 7.7 & 190 & 54 & 14 \\
\hline --do-- & $12-16-75$ & 200 & 1028 & 1028 & -- & 1,920 & 7.5 & 290 & 80 & 21 \\
\hline 97N64W10BADC & $08-01-68$ & 240 & 1028 & 46002 & 13.0 & 1,880 & 7.5 & 200 & 62 & 12 \\
\hline
\end{tabular}




\begin{tabular}{|c|c|c|c|c|c|c|c|c|c|c|c|c|}
\hline $\begin{array}{c}\text { Sodium, } \\
\text { dis- } \\
\text { aolved } \\
\text { (mg/L } \\
\text { as } \mathrm{Na}) \\
(00930)\end{array}$ & $\begin{array}{l}\text { Potas- } \\
\text { sium, } \\
\text { dis- } \\
\text { solved } \\
\text { (mg/L } \\
\text { as K) } \\
(00935)\end{array}$ & $\begin{array}{c}\text { Chloride, } \\
\text { dia- } \\
\text { solved } \\
\text { (mg/L } \\
\text { as Cl) } \\
(00940)\end{array}$ & $\begin{array}{c}\text { Sulfate, } \\
\text { dia- } \\
\text { solved } \\
\text { (mg/L } \\
\left.\text { as } \mathrm{SO}_{4}\right) \\
(00945)\end{array}$ & $\begin{array}{c}\text { Fluoride, } \\
\text { dis- } \\
\text { solved } \\
\text { (mg/L } \\
\text { as F) } \\
(00950)\end{array}$ & $\begin{array}{c}\text { Silica, } \\
\text { dis- } \\
\text { solved } \\
\left(\mathrm{mgll}^{2} \mathbf{s s}\right. \\
\left.\mathrm{SiO}_{2}\right) \\
(00955)\end{array}$ & $\begin{array}{c}\text { Bicar- } \\
\text { bonate } \\
\text { FET- } \\
\text { FLD } \\
\left(\mathrm{mg}^{2} \text { L as }\right. \\
\left.\mathrm{HCO}_{3}\right) \\
(00440)\end{array}$ & $\begin{array}{c}\text { Carbo- } \\
\text { nate } \\
\text { FET- } \\
\text { FLD } \\
\left(\mathrm{mg}^{\prime} / \mathrm{L} \text { as }\right. \\
\left.\mathrm{CO}_{3}\right) \\
(00445)\end{array}$ & $\begin{array}{c}\text { Nitro- } \\
\text { gen, } \\
\text { nitrata } \\
\text { total } \\
(\mathrm{mg} / \mathrm{L} \\
\text { as N) } \\
(00620)\end{array}$ & $\begin{array}{c}\text { Solids, } \\
\text { sum of } \\
\text { consti- } \\
\text { tuants, } \\
\text { dis- } \\
\text { solved } \\
(\mathrm{mg} / \mathrm{L}) \\
(70301)\end{array}$ & $\begin{array}{c}\text { Iron, } \\
\text { total } \\
\text { recover- } \\
\text { able } \\
(\mu \mathrm{g} / \mathrm{L} \\
\text { ss Fe) } \\
(01045)\end{array}$ & $\begin{array}{l}\text { Manga- } \\
\text { nese, } \\
\text { total } \\
\text { recover- } \\
\text { able } \\
(\mu g / L \\
\text { as Mn) } \\
(01055)\end{array}$ & $\begin{array}{c}\text { Boron, } \\
\text { dis- } \\
\text { solved } \\
(\mu g / L \\
\text { as B) } \\
(01020)\end{array}$ \\
\hline
\end{tabular}

\begin{tabular}{|c|c|c|c|c|c|c|c|c|c|c|c|c|}
\hline 200 & -- & 18 & 660 & 0.42 & -- & -- & -- & -- & -. & $<50$ & 1,700 & -- \\
\hline 69 & 13 & 20 & 370 & -- & -- & 370 & 0 & -- & 851 & -- & -- & -- \\
\hline -- & -- & .0 & 290 & -- & -- & -- & -- & -- & -- & -- & -- & -- \\
\hline 77 & 18 & 7.0 & -- & -- & -- & - & -- & -- & -. & 780 & -- & -- \\
\hline 52 & 14 & 20 & 430 & -- & -- & 310 & -- & -- & 889 & -- & -- & -- \\
\hline 190 & 30 & 70 & 960 & -. & -- & 290 & -- & -- & 1,790 & -- & -- & -- \\
\hline 190 & 30 & 70 & 950 & -. & .. & .. & -- & 0.800 & 1,780 & 80 & -- & -- \\
\hline 220 & 32 & 72 & 1,100 & -- & -- & -- & -- & ND & 1,950 & 20 & -- & 1,700 \\
\hline 300 & 38 & 120 & 1,100 & -- & -- & 440 & -- & .020 & 2,280 & 10 & -- & 1,500 \\
\hline 280 & -- & 250 & 920 & .31 & -- & -- & -- & -- & -- & 7,000 & 1,200 & -. \\
\hline 120 & 30 & 12 & 750 & -- & -- & 480 & -- & -- & 1,480 & -- & -- & -- \\
\hline 63 & 12 & 10 & -- &.- & -- & -- & -- & -- & -. & -- & -- & -- \\
\hline 390 & 27 & 150 & 900 & -- & - & 370 & -- & -- & 1,890 & -- & -- & -- \\
\hline 280 & -- & 11 & 2,000 & .76 & -- & -- & -- & -- & -- & -- & -- & -- \\
\hline 60 & 16 & 12 & 890 & $\cdots$ & -- & -. & -. & .040 & 1,470 & 110 & -- & 120 \\
\hline 110 & - & 20 & 860 & .21 & -- & - & -- & $<.500$ & -- & 2,900 & 4,100 & -- \\
\hline 240 & 21 & 160 & 410 & -- & -- & 280 & -- & - & 1,100 & -- & -- & -- \\
\hline 42 & 16 & 9.0 & 1,200 & -- & -- & -. & ND & -- & 1,710 & -- & .- & 220 \\
\hline 450 & 25 & 150 & -- & -- & -- & -. & .. & -- & .- & -- & -- & -- \\
\hline 280 & 23 & 130 & 560 & -- & -- & 210 & -- & -- & 1,210 & -- & -- & -- \\
\hline 340 & 31 & 170 & 670 &.- & -- & 220 & ND & -- & 1,410 & - & -- & 2,700 \\
\hline 330 & 28 & 52 & 610 & -- & -- & 260 & -- & 2.45 & 1,200 & 1,100 & -. & 200 \\
\hline 290 & 23 & 86 & 1,000 & -- & -- & 160 & -- & -- & 1,760 & -- & -- & -- \\
\hline 250 & 17 & 120 & 770 & -- & -- & -. & -- & .560 & 1,400 & 28,000 & -- & 1,200 \\
\hline 260 & 20 & 170 & 600 & -- & -- & 93 & -- & .130 & 1,280 & 94,000 & -- & 1,000 \\
\hline 250 & -- & 150 & 680 & .46 & -- & -- & -- & -- & -- & -- & 2,300 & -. \\
\hline 450 & 24 & 200 & -- & -- & -. & -- & -- & -- & -- & -- & -- & -- \\
\hline 340 & 22 & 210 & 480 & -- & -- & 140 & -- & -- & 1190 & -- & -- & -- \\
\hline 300 & 25 & 230 & 490 & -- & -- & 230 & -- & -- & 1,310 & -- & -- & -- \\
\hline 340 & 30 & 180 & 450 & -- & -- & 460 & $\cdots$ & -- & 1,370 & -- & -- & -- \\
\hline 280 & 24 & 36 & 1,000 & -- & -- & 250 & -- & 1.90 & 1,770 & 2,400 & -- & 2,900 \\
\hline 320 & 18 & 140 & -- & -- & -- & $\cdots$ & -- & -- & -- & 30 & -- & -- \\
\hline 450 & 22 & 240 & -- & - & -- & -- & -. & -- & -- & -- & -- & -- \\
\hline 330 & 26 & 80 & 590 & -- & -- & 330 & -. & -- & 1,290 & -- & -- & -- \\
\hline 320 & 18 & 140 & 500 & - & -- & 250 & -- & -- & 1,180 & -- & -- & 1,400 \\
\hline
\end{tabular}


Table 3. Chemical analyses of ground water in Charles Mix and Douglas Counties--Continued

\begin{tabular}{|c|c|c|c|c|c|c|c|c|c|c|}
\hline $\begin{array}{l}\text { Principal aquifer/ } \\
\text { local Identifier }\end{array}$ & $\begin{array}{l}\text { Dete of } \\
\text { sample }\end{array}$ & $\begin{array}{c}\text { Depth } \\
\text { of } \\
\text { well } \\
\text { (feet) } \\
\text { (00003) }\end{array}$ & $\begin{array}{c}\text { Agency } \\
\text { collecting } \\
\text { sample } \\
\text { (code } \\
\text { number) } \\
\text { (00027) }\end{array}$ & $\begin{array}{c}\text { Agency } \\
\text { analyzing } \\
\text { sample } \\
\text { (code } \\
\text { number) } \\
\text { (00028) }\end{array}$ & $\begin{array}{c}\text { Water } \\
\text { temper- } \\
\text { ature } \\
\left({ }^{\circ} \mathrm{C}\right) \\
(00010)\end{array}$ & $\begin{array}{c}\text { Specific } \\
\text { conduct- } \\
\text { ance } \\
(\mu \mathrm{S} / \mathrm{cm}) \\
(00095)\end{array}$ & $\begin{array}{c}\text { pH } \\
\text { (standard } \\
\text { units) } \\
(00400)\end{array}$ & $\begin{array}{c}\text { Hardness } \\
\text { totel } \\
\text { (mg/L as } \\
\left.\mathrm{CaCO}_{3}\right) \\
(00900)\end{array}$ & $\begin{array}{l}\text { Calcium, } \\
\text { dissolved } \\
\text { (mg/h } \\
\text { as Ca) } \\
(00915)\end{array}$ & $\begin{array}{l}\text { Magne- } \\
\text { sium, } \\
\text { dissolved } \\
\text { (mg/l } \\
\text { as Mg) } \\
(00925)\end{array}$ \\
\hline
\end{tabular}

Choteau aquifer (Cont.)

\begin{tabular}{|c|c|c|c|c|c|c|c|c|c|c|}
\hline 97N64W10CDBA & $08-09-67$ & 206 & 1028 & 46002 & 13.5 & 2,450 & 7.6 & 370 & 110 & 24 \\
\hline 97N64W11BAAA & $10-02-69$ & 221 & 1028 & 46002 & 12.0 & 2,230 & 8.4 & 250 & 46 & 33 \\
\hline --do-- & $10-09-69$ & 221 & 1028 & 1028 & -- & 2,230 & 8.4 & 240 & 40 & 33 \\
\hline --do-- & $06-13-74$ & 221 & 1028 & 1028 & -- & -- & -- & -- & -- & -- \\
\hline --do-- & $08-22-79$ & 221 & 1028 & 1028 & 23.0 & 2,220 & -- & 150 & 43 & 10 \\
\hline 97N64W11CACB & $02-22-57$ & 200 & 1028 & 46001 & 11.0 & 1,500 & 7.8 & 200 & 53 & 16 \\
\hline --do-- & $03-03-57$ & 200 & 1028 & 46001 & -- & 1,500 & 8.1 & 150 & 42 & 12 \\
\hline --do-- & $07-14-67$ & 200 & 1028 & 46002 & -- & 2,220 & 8.0 & 220 & 80 & 5.0 \\
\hline --do-- & $08-01-67$ & 200 & 1028 & 46002 &.- & 1,780 & 7.6 & 180 & 58 & 9.0 \\
\hline --do-- & $08-08-67$ & 200 & 1028 & 46002 & 11.0 & 3,260 & 7.9 & 830 & 150 & 110 \\
\hline --do-- & $08-17-67$ & 200 & 1028 & 46002 & -- & 1,990 & 7.6 & 180 & 62 & 6.0 \\
\hline 97N64WI3AAC & $04-12-79$ & 220 & 1028 & 1028 & -- & 1,880 & 7.3 & 380 & 120 & 20 \\
\hline 97N64W14BBBB & $08-02-68$ & 209 & 1028 & 46002 & 12.0 & 1,900 & 7.9 & 210 & 64 & 12 \\
\hline --do-- & $10-16-69$ & 209 & 1028 & 1028 & - & 1,820 & 7.8 & 200 & 61 & 12 \\
\hline --do-- & $08-22-79$ & 209 & 1028 & 1028 & 23.0 & 1,850 & -- & 190 & 56 & 11 \\
\hline 97N64W20CCA & $-\quad-75$ & 200 & 1028 & 1028 & -- & 2,300 & 7.5 & 210 & 53 & 19 \\
\hline --do-- & $-\quad-77$ & 200 & 1028 & 1028 & -- & 3,150 & 6.9 & 590 & 150 & 54 \\
\hline 97N64W20CCA2 & $02-26-75$ & 202 & 1028 & 1028 & -- & 2,300 & 7.5 & 210 & 53 & 19 \\
\hline 97N64W20CDCD & $10-03-69$ & 178 & 1028 & 46002 & 12.0 & 2,840 & 8.4 & 560 & 110 & 69 \\
\hline --do-- & $10-09-69$ & 178 & 1028 & 1028 & -- & 2,840 & 8.4 & 560 & 110 & 69 \\
\hline --do-- & $06-13-74$ & 178 & 1028 & 1028 & - & 2,810 & 7.5 & 390 & 100 & 34 \\
\hline --do-- & $08-22-79$ & 178 & 1028 & 1028 & 23.0 & 2,490 & - & 390 & 110 & 29 \\
\hline $97 \mathrm{~N} 64 \mathrm{~W} 22 \mathrm{CBCC}$ & $10-02-69$ & 210 & 1028 & 46002 & 12.0 & 2,640 & 7.6 & 760 & 160 & 88 \\
\hline --do-- & $06-13-74$ & 210 & 1028 & 1028 & -- & 2,720 & 7.7 & 660 & 180 & 50 \\
\hline-- do-- & $08-22-79$ & 210 & 1028 & 1028 & 23.0 & 2,600 & - & 600 & 170 & 45 \\
\hline 97N64W25DDB & -.74 & 180 & 1028 & 1028 & -- & 2,320 & 7.8 & 340 & 95 & 26 \\
\hline --do-- & $02-19-75$ & 180 & 1028 & 1028 & -- & 1,910 & 8.3 & 220 & 62 & 16 \\
\hline 97N64W27DACB & $05-26-66$ & 260 & 1028 & 1028 & -- & 2,620 & 7.7 & 460 & 130 & 33 \\
\hline --do-- & $07-08-66$ & 260 & 1028 & 1028 & -- & 2,140 & 7.6 & 440 & 130 & 29 \\
\hline --do- & $07-14-67$ & 260 & 1028 & 1028 & -- & 2,380 & 7.5 & 470 & 130 & 36 \\
\hline --do-- & $08-01-67$ & 260 & 1028 & 1028 & -- & 2,360 & 7.5 & 440 & 130 & 27 \\
\hline --do-- & $08-24-67$ & 260 & 1028 & 46002 & 13.5 & 2,620 & 7.4 & 450 & 130 & 30 \\
\hline 97N64W27DDDD & $09-21-67$ & 250 & 1028 & 46002 & 12.0 & 2,620 & 8.0 & 490 & 150 & 29 \\
\hline --do-- & $08-22-79$ & 250 & 1028 & 1028 & 25.0 & 2,450 & -- & 450 & 120 & 35 \\
\hline 97N64W31BAAA & $08-02-68$ & 188 & 1028 & 46002 & 12.0 & 2,780 & 8.1 & 400 & 120 & 24 \\
\hline
\end{tabular}




\begin{tabular}{|c|c|c|c|c|c|c|c|c|c|c|c|c|}
\hline $\begin{array}{l}\text { Sodium, } \\
\text { dis- } \\
\text { solved } \\
\text { (mg/L } \\
\text { as } \mathrm{Na}) \\
(00930)\end{array}$ & $\begin{array}{l}\text { Potas- } \\
\text { sium, } \\
\text { dis- } \\
\text { solved } \\
\text { (mg/L } \\
\text { as K) } \\
(00935)\end{array}$ & $\begin{array}{c}\text { Chloride, } \\
\text { dis- } \\
\text { solved } \\
\text { (mg/L } \\
\text { as CI) } \\
\text { (00940) }\end{array}$ & $\begin{array}{c}\text { Sulfate, } \\
\text { dis- } \\
\text { solved } \\
\text { (mg/L } \\
\left.\text { as } \mathrm{SO}_{4}\right) \\
(00945)\end{array}$ & $\begin{array}{c}\text { Fluoride, } \\
\text { dis- } \\
\text { solved } \\
\text { (mg/L } \\
\text { ss F) } \\
(00950)\end{array}$ & $\begin{array}{c}\text { Silica, } \\
\text { dis- } \\
\text { solved } \\
(\mathrm{mg} / \mathrm{l} \text { ss } \\
\left.\mathrm{SiO}_{2}\right) \\
(00955)\end{array}$ & $\begin{array}{c}\text { Bicar- } \\
\text { bonste } \\
\text { FET- } \\
\text { FLD } \\
\left(\mathrm{mgll}^{2} \text { as }\right. \\
\left.\mathrm{HCO}_{3}\right) \\
(00440)\end{array}$ & $\begin{array}{c}\text { Csrbo- } \\
\text { nate } \\
\text { FET- } \\
\text { FLD } \\
(\mathrm{mg} / \mathrm{L} \text { as } \\
\left.\mathrm{CO}_{3}\right) \\
(00445)\end{array}$ & $\begin{array}{c}\text { Nitro- } \\
\text { gen, } \\
\text { nitrate } \\
\text { totsl } \\
(\mathrm{mg} / \mathrm{L} \\
\text { as N) } \\
(00620)\end{array}$ & $\begin{array}{l}\text { Solids, } \\
\text { sum of } \\
\text { constj- } \\
\text { tuents, } \\
\text { dis- } \\
\text { solved } \\
\text { (mg/L) } \\
\text { (70301) }\end{array}$ & $\begin{array}{l}\text { Iron, } \\
\text { total } \\
\text { recover- } \\
\text { sble } \\
(\mu g / L \\
\text { as Fe) } \\
(01045)\end{array}$ & $\begin{array}{l}\text { Msngs- } \\
\text { nese, } \\
\text { total } \\
\text { recover- } \\
\text { able } \\
(\mu g / L \\
\text { as Mn) } \\
(01055)\end{array}$ & $\begin{array}{c}\text { Boron, } \\
\text { dis- } \\
\text { solved } \\
(\mu \mathrm{g} / \mathrm{L} \\
\text { as B) } \\
(01020)\end{array}$ \\
\hline
\end{tabular}

\begin{tabular}{|c|c|c|c|c|c|c|c|c|c|c|c|c|}
\hline 350 & 24 & 190 & 560 & -. & -- & 330 & -- & -- & 1,420 & - & -- & 1,900 \\
\hline 460 & 21 & 230 & 430 & -- & -- & 290 & -- & -- & 1,360 & -- & -- & -- \\
\hline 460 & 21 & 230 & 430 & -- & -- & -- & -- & ND & 1,380 & ND & -- & -- \\
\hline-- & -- & -- & -- & -- & -- & -- & -- & -- & - & - & -- & -- \\
\hline 420 & -- & 410 & 360 & 1.0 & -- & -- & -- & -. & -- & $<50$ & 750 & -- \\
\hline 400 & 26 & 260 & 360 & -- & -- & 380 & 0 & -. & 1,300 & -- & -- & -- \\
\hline 460 & 25 & 210 & 450 & -- & -- & 380 & 16 & -- & 1,400 & -- & -- & -- \\
\hline 430 & 27 & 190 & 460 & -- & - & 370 & -. & -- & 1,370 & - & -- & -- \\
\hline 400 & 17 & 200 & 350 & -- & -- & 370 & -- & - & 1,220 & - & -- & -- \\
\hline 450 & 24 & 210 & 1,000 & -- & -- & 410 & -- & - & 2,150 & -- & $\overrightarrow{-}$ & 2,000 \\
\hline 390 & 9.0 & 200 & 350 & -- & - & 380 & -- & -- & 1,200 & -- & -. & -- \\
\hline 290 & 31 & 130 & 590 & -. & - & 240 & - & -- & 1,310 & -- & - & -- \\
\hline 360 & 21 & 140 & 500 & -- & -- & 220 & -- & -- & 1,210 & -- & -- & -- \\
\hline 310 & 20 & 150 & 530 & -- & -- & -- & - & ND & 1,210 & ND & -. & 2,600 \\
\hline 320 & -- & 350 & 300 & .54 & -- & .. & -- & -- & -- & $<50$ & 1,000 & -- \\
\hline 480 & 20 & 290 & -- & -. & -- & - & -- & -- & -- & - & -- & -- \\
\hline 500 & 31 & 330 & -- & -- & -- & -- & -- & -- & -- & - & -- & -- \\
\hline 480 & 20 & 290 & 390 & -- & -- & 410 & - & -. & 1,450 & - & -- & -- \\
\hline 650 & 29 & 250 & 760 & -- & -- & 370 & - & -- & 2,050 & -- & -- & 4,200 \\
\hline 650 & 29 & 250 & 760 & -- & -- & -- & - & 2.80 & 2,090 & 320 & -- & 4,300 \\
\hline 570 & 34 & 260 & 650 & -- & -- & 440 & -- & .100 & 1,870 & ND & -- & 3,300 \\
\hline 470 & -- & 400 & 530 & .42 & -- & -- & -- & -- & -- & $<50$ & 1,700 & -- \\
\hline 320 & 30 & 170 & 780 & -- & -- & 410 & -- & -- & 1,750 & -- & -- & -- \\
\hline 450 & 37 & 190 & 930 & -- & -- & 400 & -- & .050 & 2,040 & 10 & -- & 2,700 \\
\hline 370 & -- & 350 & 660 & .30 & -- & -- & -- & -- & -- & 170 & 2,400 & -- \\
\hline 450 & 32 & 170 & -- & -- & -- & -- & -- & - & -- & - & -- & -- \\
\hline 390 & 31 & 160 & 580 & -- & -- & 120 & 14 & - & 1,310 & -- & -- & -- \\
\hline 440 & 35 & 150 & 790 & -- & -- & 340 & -- & - & 1,750 & -- & -- & -- \\
\hline 340 & 28 & 140 & 820 & -- & - & 300 & -- & -- & 1,630 & -- & -- & -- \\
\hline 430 & 29 & 160 & 710 & -- & - & 360 & -- & -- & 1,670 & -- & -- & -- \\
\hline 400 & 26 & 160 & 680 & -- & - & 340 & -- & -- & 1,590 & - & -- & -- \\
\hline 410 & 26 & 180 & 690 & -- & - & 300 & -- & -- & 1,610 & -- & -. & 2,200 \\
\hline 360 & 27 & 160 & 750 & -- & - & 280 & -- & -- & 1,610 & -- & -- & - \\
\hline 380 & -- & 170 & 690 & .35 & - & -- & -- & - & -- & $<50$ & 1,900 & -- \\
\hline 460 & 27 & 190 & 740 & - & -- & 390 & -. & -- & 1,750 & -- & -- & - \\
\hline
\end{tabular}


Table 3. Chemical analyses of ground water in Charles Mix and Douglas Counties--Continued

\begin{tabular}{|c|c|c|c|c|c|c|c|c|c|c|}
\hline $\begin{array}{l}\text { Principal aquifer/ } \\
\text { local identifier }\end{array}$ & $\begin{array}{l}\text { Date of } \\
\text { sample }\end{array}$ & $\begin{array}{c}\text { Depth } \\
\text { of } \\
\text { well } \\
\text { (feet) } \\
\text { (00003) }\end{array}$ & $\begin{array}{c}\text { Agency } \\
\text { collecting } \\
\text { sample } \\
\text { (code } \\
\text { number) } \\
\text { (00027) }\end{array}$ & $\begin{array}{c}\text { Agency } \\
\text { analyzing } \\
\text { sample } \\
\text { (code } \\
\text { number) } \\
\text { (00028) }\end{array}$ & $\begin{array}{c}\text { Water } \\
\text { temper- } \\
\text { ature } \\
\left({ }^{\circ} \mathrm{C}\right) \\
(00010)\end{array}$ & $\begin{array}{c}\text { Specific } \\
\text { conduct- } \\
\text { ance } \\
(\mu S / c m) \\
(00095)\end{array}$ & $\begin{array}{c}\text { pH } \\
\text { (stendard } \\
\text { units) } \\
(00400)\end{array}$ & $\begin{array}{c}\text { Hardness } \\
\text { total } \\
(\mathrm{mg} / \mathrm{L} \text { as } \\
\left.\mathrm{CeCO}_{3}\right) \\
(00900)\end{array}$ & $\begin{array}{l}\text { Calcium, } \\
\text { dissolved o } \\
\text { (mg/L } \\
\text { as Ca) } \\
(00915)\end{array}$ & $\begin{array}{l}\text { Magne- } \\
\text { sium, } \\
\text { dissolved } \\
\text { ( } \mathrm{mg} / \mathrm{L} \\
\text { as Mg) } \\
\text { (00925) }\end{array}$ \\
\hline
\end{tabular}

Choteau aquifer (Cont.)

\begin{tabular}{|c|c|c|c|c|c|c|c|c|c|c|}
\hline 97N64W31BAAA & $10-16-69$ & 188 & 1028 & 1028 & -- & 2,620 & 8.1 & 360 & 100 & 26 \\
\hline --do-- & $08-24-79$ & 188 & 1028 & 1028 & 25.0 & 2,600 & -- & 340 & 90 & 27 \\
\hline 97N64W31CCCC & $06-\quad-65$ & 160 & 46008 & 46008 & -- & - & 7.5 & 510 & -- & 27 \\
\hline 97N64W32DDCC & $-\quad-65$ & 240 & 46008 & 46008 & -- & -- & 7.6 & 60 & 11 & 8.0 \\
\hline 97N64W33ABBC & $11-14-80$ & 240 & 1028 & 1028 & - & 3,350 & 7.3 & 930 & 230 & 86 \\
\hline 97N64W35BCAA & $07-14-67$ & 262 & 1028 & 46002 & -- & 2,560 & 7.6 & 430 & 84 & 53 \\
\hline --do-- & $08-01-67$ & 262 & 1028 & 46002 & -- & 2,140 & 7.5 & 380 & 120 & 20 \\
\hline --do-- & $08-16-67$ & 262 & 1028 & 46002 & 12.0 & 2,480 & 8.1 & 320 & 93 & 22 \\
\hline --do-- & $08-17-67$ & 262 & 1028 & 46002 & -- & 2,390 & 7.5 & 390 & 130 & 16 \\
\hline 97N64W35DADA & $08-24-67$ & 221 & 1028 & 1028 & -- & 2,360 & 8.1 & 370 & 130 & 12 \\
\hline --do-- & $08-22-79$ & 221 & 1028 & 1028 & 25.0 & 2,000 & -- & 330 & 95 & 23 \\
\hline 97N65W $13 \mathrm{CBBC}$ & $-\quad-65$ & 200 & 46008 & 46008 & -- & -- & 7.4 & 490 & 130 & 41 \\
\hline 97N65W13DDDD & $06-21-67$ & 162 & 1,028 & 46002 & 16.0 & 2,120 & 7.7 & 600 & 150 & 53 \\
\hline --do-- & $10-16-69$ & 162 & 1028 & 1028 & - & 2,200 & 8.1 & 550 & 130 & 54 \\
\hline --do-- & $06-13-74$ & 162 & 1028 & 1028 & -- & 2,280 & 7.9 & 660 & 150 & 69 \\
\hline --do-- & $08-24-79$ & 162 & 1028 & 1028 & 21.0 & 1,660 & -- & 540 & 120 & 56 \\
\hline 97N65W31DDDC & $-\quad-65$ & 260 & 46008 & 46008 & $\cdots$ & -- & 8.0 & 670 & 230 & 26 \\
\hline 97N65W32DDDA & $-\quad-65$ & 180 & 46008 & 46008 & -- & -- & 8.2 & 470 & 180 & 5.0 \\
\hline 97N65W34CCCC & $06-21-67$ & 160 & 1028 & 46002 & 16.0 & 3,070 & 7.4 & 1,000 & 160 & 150 \\
\hline --do-- & $09-19-68$ & 160 & 1028 & 1028 & -- & 2,910 & 8.0 & 930 & 220 & 92 \\
\hline-- do-- & $07-10-73$ & 160 & 1028 & 1028 & -- & 5,310 & 7.5 & 1,700 & 430 & 160 \\
\hline 97N65W35AAAA & $08-20-79$ & 223 & 1028 & 1028 & 21.0 & 3,510 & -- & 680 & 180 & 55 \\
\hline $98 \mathrm{~N} 62 \mathrm{~W} 2 \mathrm{ABBB}$ & $09-09-68$ & 320 & 1028 & 46002 & -- & 1,580 & 8.1 & 270 & 86 & 13 \\
\hline $98 \mathrm{~N} 62 \mathrm{~W} 3 \mathrm{CCCC}$ & $05-15-80$ & 318 & 1028 & 46002 & -- & 1,800 & 7.6 & 460 & 130 & 34 \\
\hline 98N62W 9DABC & $05-16-77$ & 270 & 1028 & 1028 & -- & 1,990 & 7.6 & 400 & 110 & 29 \\
\hline $98 \mathrm{~N} 63 \mathrm{~W} 22 \mathrm{C}$ & $00-00-68$ & 200 & 1028 & 1028 & -- & 2,470 & 7.9 & 170 & 54 & 9.0 \\
\hline 98N63W24BBBB & $05-15-80$ & 223 & 1028 & 1028 & -- & 2,050 & 7.8 & 580 & 170 & 38 \\
\hline $98 \mathrm{~N} 63 \mathrm{~W} 26 \mathrm{CCAA}$ & $01-27-75$ & 215 & 1028 & 1028 & -- & 2,600 & 7.2 & 420 & 120 & 28 \\
\hline 98N63W28BCC & $10-15-57$ & 265 & 46008 & 46001 & -- & 2,200 & 7.8 & 180 & 44 & 16 \\
\hline 98N63W29DDAD & $10-09-69$ & 199 & 1028 & 1028 & - & 2,180 & 8.3 & 220 & 46 & 25 \\
\hline --do-- & $06-13-74$ & 199 & 1028 & 1028 & -- & 2,170 & 7.9 & 190 & 53 & 14 \\
\hline --do-- & $08-23-79$ & 199 & 1028 & 1028 & 23.0 & 2,150 & -- & 140 & 42 & 9.0 \\
\hline 98N63W31CCD & $08-06-76$ & 220 & 1028 & 1028 & -- & 2,530 & 7.4 & 590 & 170 & 40 \\
\hline 98N63W31DDDD & $08-10-78$ & 225 & 46002 & 46002 & -- & 2,110 & 7.9 & 300 & 88 & 19 \\
\hline --do-- & $08-21-79$ & 225 & 1028 & 1028 & 22.0 & 2,080 & -- & 260 & 72 & 19 \\
\hline
\end{tabular}




\begin{tabular}{|c|c|c|c|c|c|c|c|c|c|c|c|c|}
\hline $\begin{array}{c}\text { Sodium, } \\
\text { dis- } \\
\text { solved } \\
\text { (mg/h } \\
\text { as } \mathrm{Na}) \\
(00930)\end{array}$ & $\begin{array}{l}\text { Potas- } \\
\text { sium, } \\
\text { dis- } \\
\text { solved } \\
\text { (mg/L } \\
\text { as K) } \\
(00935)\end{array}$ & $\begin{array}{c}\text { Chloride, } \\
\text { dis- } \\
\text { solved } \\
\text { (mg/l } \\
\text { as } \mathrm{Cl} \text { ) } \\
(00940)\end{array}$ & $\begin{array}{c}\text { Sulfate, } \\
\text { dis- } \\
\text { solved } \\
\text { (mg/l } \\
\left.\mathrm{as} \mathrm{SO}_{4}\right) \\
(00945)\end{array}$ & $\begin{array}{c}\text { Fluoride, } \\
\text { dis- } \\
\text { solved } \\
\text { (mg/L } \\
\text { as F) } \\
\text { (00950) }\end{array}$ & $\begin{array}{c}\text { Silica, } \\
\text { dis- } \\
\text { solved } \\
\left(\mathrm{mg}^{2} \mathrm{l} \text { as }\right. \\
\left.\mathrm{SiO}_{2}\right) \\
(00955)\end{array}$ & $\begin{array}{c}\text { Bicar- } \\
\text { bonate } \\
\text { FET- } \\
\text { FLD } \\
\text { (mgllas } \\
\left.\mathrm{HCO}_{3}\right) \\
(00440)\end{array}$ & $\begin{array}{c}\text { Carbo- } \\
\text { nate } \\
\text { FET- } \\
\text { FLD } \\
(\mathrm{mg} / \mathrm{l} \text { as } \\
\left.\mathrm{CO}_{3}\right) \\
(00445)\end{array}$ & $\begin{array}{c}\text { Nitro- } \\
\text { gen, } \\
\text { nitrate } \\
\text { total } \\
\text { (mg/L } \\
\text { as N) } \\
(00620)\end{array}$ & $\begin{array}{l}\text { Solids, } \\
\text { sum of } \\
\text { consti- } \\
\text { tuents, } \\
\text { dis- } \\
\text { solved } \\
\text { (mg/l) } \\
\text { (70301) }\end{array}$ & $\begin{array}{c}\text { Iron, } \\
\text { total } \\
\text { recover- } \\
\text { able } \\
(\mu \mathrm{g} / \mathrm{L} \\
\text { as Fe) } \\
(01045)\end{array}$ & $\begin{array}{c}\text { Manga- } \\
\text { nese, } \\
\text { total } \\
\text { recover- } \\
\text { able } \\
(\mu g / L \\
\text { as Mn) } \\
(01055)\end{array}$ & $\begin{array}{c}\text { Boron, } \\
\text { dis- } \\
\text { solved } \\
(\mu \mathrm{g} / \mathrm{L} \\
\text { as } \mathrm{B}) \\
(01020)\end{array}$ \\
\hline
\end{tabular}

\begin{tabular}{|c|c|c|c|c|c|c|c|c|c|c|c|c|}
\hline 310 & 26 & 200 & 650 & -- & - & -- & -- & 2.20 & 1,500 & 40 & -- & 3,800 \\
\hline 510 & -- & 180 & 790 & 0.63 & - & - & -- & -- & -- & 550 & 770 & -- \\
\hline -- & -- & 150 & 970 & -- & -- & -- & -- & -- & -- & 1,800 & -- & -- \\
\hline -- & - & 1,100 & .0 & -- & -- & - & -- & -- & -- & -- & -- & -- \\
\hline 420 & 37 & 410 & 1,000 & -- & -- & 420 & -- & -- & 2,410 & -- & -- & -- \\
\hline 430 & 26 & 210 & 620 & - & -- & 300 & -- & -. & 1,570 & -- & -- & -- \\
\hline 420 & 25 & 190 & 630 & -- & -- & 310 & -- & -- & 1,560 & -- & - & -- \\
\hline 440 & 26 & 200 & 650 & -- & -. & 250 & -- & -- & 1,550 & -- & -- & 1,900 \\
\hline 400 & 13 & 200 & 630 & -- & -. & 310 & -- & -- & 1,540 & -- & -- & -- \\
\hline 370 & 24 & 220 & 490 & -- & -- & -- & -- & 1.65 & 1,390 & 8,700 & -- & -- \\
\hline 350 & -- & 230 & 460 & .42 & -- & -- & -- & -- & -- & $<50$ & 1,300 & -- \\
\hline -- & -- & 260 & 550 & -- & -- & -- & -- & -. & 2,110 & .. & -- & -- \\
\hline 260 & 22 & 22 & 710 & -- & - & 200 & -- & -- & 1,310 & -. & -- & -- \\
\hline 280 & 22 & 25 & 1,000 & -. & -- & -- & -. & 1.31 & 1,620 & 10 & -- & 2,100 \\
\hline 290 & 26 & 19 & 1,100 & -- & -- & 180 & -- & .010 & 1,750 & ND & -- & 1,500 \\
\hline 270 & -- & 19 & 900 & .41 & -- & -- & $\cdots$ & -- & -- & $<50$ & 750 & $\cdots$ \\
\hline -- & -- & 150 & 1,100 & $\cdots$ & -. & -- & -- & -- & 2,060 & -- & -- & -- \\
\hline -- & -- & 150 & 1,100 & -- & -- & -- & -- & -- & 2,040 & -- & -- & - \\
\hline 370 & 27 & 34 & 1,300 & - & -- & 390 & + & -- & 2,230 & -- & -- & -. \\
\hline 370 & 24 & 40 & 1,300 & .40 & 21 & 390 & ND & .100 & 2,310 & 400 & 1,400 & 1,100 \\
\hline 540 & 26 & 18 & 2,700 & -- & -- & 420 & -- & .150 & 4,030 & 70 & -- & 780 \\
\hline 490 & -- & 160 & 1,000 & .52 & -- & -- & -- & -- & -- & $<50$ & 2,600 & -- \\
\hline 260 & 18 & 77 & 670 & -- & - & 110 & -- & $\cdots$ & 1,180 & -- & -- & -- \\
\hline 300 & 23 & 120 & 860 & -- & -- & 110 & -- & 2.20 & 1,520 & 1,500 & -- & 2,000 \\
\hline 310 & 25 & 98 & 850 & -- & -- & 180 & -- & -- & 1,510 & -- & -- & - \\
\hline 470 & 30 & 280 & -- & -- & -- & -- & -- & -- & -- & 30 & -- & -. \\
\hline 280 & 31 & 160 & 870 & -- & -- & 120 & -- & 1.98 & 1,610 & 680 & -- & 2,100 \\
\hline 480 & 32 & 310 & 560 & -- & -- & 400 & -- & -- & 1,720 & - & -- & -- \\
\hline 500 & 13 & 140 & 790 & -- & -- & 320 & 3 & -- & 1,660 & -- & -- & -- \\
\hline 520 & 21 & 120 & 560 & -- & -- & -- & -- & .660 & 1,460 & 1,300 & -- & -- \\
\hline 480 & 23 & 120 & 660 & -- & -- & 340 & -- & ND & 1,520 & 10 & -- & 3,700 \\
\hline 420 & -- & 220 & 490 & .82 & -- & -- & -- & -- & -- & $<50$ & 860 & -- \\
\hline 340 & 42 & 63 & 1,000 & -- & -- & 300 & -- & - & 1,830 & - & -- & -- \\
\hline 350 & 32 & 110 & 660 & -- & - & 310 & - & 2.80 & 1,410 & 460 & - & 3,700 \\
\hline 370 & -- & 110 & 590 & .59 & -- & -- & -- & -- & -- & $<50$ & 1,200 & -- \\
\hline
\end{tabular}


Table 3. Chemical analyses of ground water in Charles Mix and Douglas Counties--Continued

\begin{tabular}{|c|c|c|c|c|c|c|c|c|c|c|}
\hline $\begin{array}{l}\text { Principal aquifer/ } \\
\text { local identifier }\end{array}$ & $\begin{array}{l}\text { Date of } \\
\text { sampla }\end{array}$ & $\begin{array}{l}\text { Depth } \\
\text { of } \\
\text { well } \\
\text { (feet) } \\
(00003)\end{array}$ & $\begin{array}{l}\text { Agency } \\
\text { collecting } \\
\text { sample } \\
\text { (code } \\
\text { number) } \\
\text { (00027) }\end{array}$ & $\begin{array}{l}\text { Agency } \\
\text { analyzing } \\
\text { sample } \\
\text { (code } \\
\text { number) } \\
\text { (00028) }\end{array}$ & $\begin{array}{c}\text { Water } \\
\text { temper- } \\
\text { ature } \\
\left({ }^{\circ} \mathrm{C}\right) \\
(00010)\end{array}$ & $\begin{array}{c}\text { Specific } \\
\text { conduct- } \\
\text { ance } \\
(\mu S / \mathrm{cm}) \\
(00095)\end{array}$ & $\begin{array}{c}\text { pH } \\
\text { (standard } \\
\text { units) } \\
(00400)\end{array}$ & $\begin{array}{c}\text { Hardness } \\
\text { total } \\
(\mathrm{mg} / \mathrm{L} \text { as } \\
\left.\mathrm{CaCO}_{3}\right) \\
(0090)\end{array}$ & $\begin{array}{c}\text { Calcium, } \\
\text { disaolvad } \\
\text { (mg/h } \\
\text { as Ca) } \\
\text { (00915) }\end{array}$ & $\begin{array}{l}\text { Magne- } \\
\text { sium, } \\
\text { dissolvad } \\
\text { (mg/ } \\
\text { as } \mathrm{Mg} \text { ) } \\
\text { (00925) }\end{array}$ \\
\hline
\end{tabular}

Choteau aquifer (Cont.)

$\begin{array}{lrrrrrrrrrr}\text { 98N63W33DCBB } & 07-31-68 & 193 & 1028 & 46002 & 14.0 & 2,220 & 7.8 & 260 & 78 & 15 \\ \text { 99N62W25DDDD } & 05-15-80 & 330 & 1028 & 1028 & -- & 2,100 & 7.7 & 1,300 & 380 & 77 \\ \text { 99N62W27BBBB } & 07-11-67 & 201 & 1028 & 46002 & 12.0 & 3,470 & 7.7 & 1,300 & 160 & 210 \\ \text {--do-- } & 08-24-67 & 201 & 1028 & 1028 & -- & 3,470 & -- & 1,300 & 160 & 210 \\ \text {--do-- } & 09-24-69 & 201 & 1028 & 1028 & -- & 3,570 & -- & 1,600 & 360 & 180 \\ & & & & & & & & & & \\ \text {--do-- } & 08-23-79 & 201 & 1028 & 1028 & 21.0 & 2,950 & -- & 1,400 & 300 & 160\end{array}$

Corsica aquifer

$\begin{array}{lrrrrrrrrrr}\text { 99N63W 7BBBB } & 05-15-80 & 260 & 1028 & 1028 & -- & 1,200 & 7.7 & 300 & 72 & 29 \\ \text { 99N64W 1B } & 11-14-77 & 250 & 1028 & 1028 & -- & 2,050 & 7.7 & 940 & 340 & 24 \\ \text { 100N64W11BBBB } & 06-10-77 & 178 & 46007 & 46007 & - & 1,190 & 8.0 & 360 & 130 & 10 \\ \quad-- \text { do-- } & 08-23-79 & 178 & 1028 & 1028 & 21.0 & 1,070 & - & 310 & 93 & 20 \\ \text { 100N64W21C } & 00-00-68 & 180 & 1028 & 1028 & -- & 2,760 & 7.8 & 910 & 320 & 29 \\ & & & & & & & & & & \\ \text { 100N64W21DAAA } & 07-31-68 & 180 & 1028 & 46002 & 11.0 & 2,760 & 7.8 & 920 & 320 & 29 \\ \quad-- \text { do-- } & 06-19-80 & 180 & 1028 & 1028 & -- & 1,800 & - & 490 & 100 & 55\end{array}$

Delmont aquifer

$\begin{array}{lrrrrrrrrrc}------ & & & & & \\ \text { 95N62W 7BBBA } & 12-18-58 & 65 & 1028 & 1028 & -- & 2,430 & 7.1 & 710 & 190 & 58 \\ \text {--do-- } & 12-18-58 & 65 & 1028 & 1028 & -- & 2,430 & 7.1 & 700 & 190 & 58 \\ \text {--do-- } & 09-18-67 & 65 & 1028 & 46002 & 11.0 & 2,480 & 7.6 & 690 & 210 & 39 \\ \text {--do-- } & 05--69 & 65 & 1028 & 1028 & -- & 2,490 & 8.0 & 36 & 10 & 2.7 \\ - \text {--do-- } & 08-24-79 & 65 & 1028 & 1028 & -- & -- & -- & -- & -- & -- \\ & & & & & & & & & & \\ \text { 95N62W34CDDC } & 06-06-67 & 44 & 1028 & 46002 & 13.0 & 1,530 & 7.4 & 800 & 220 & 60 \\ \text { 97N63W 1ABB } & 03-02-76 & 60 & 1028 & 1028 & -- & 799 & 7.6 & 290 & 68 & 28 \\ \text { 97N63W 1ABBB } & 07-02-80 & 50 & 1028 & 1028 & -- & 1,110 & 8.0 & 400 & 100 & 34 \\ \text { 97N63W 1CADD } & 12-05-79 & 43 & 1028 & 1028 & -- & 659 & 7.5 & 360 & 100 & 24 \\ \text { 97N63W 1DDB } & 02-18-75 & 50 & 1028 & 1028 & -- & 957 & 7.8 & 460 & 100 & 50 \\ & & & & & & & & & & \\ \text { 97N63W 2 } & 11-05-74 & 20 & 1028 & 1028 & -- & 351 & 7.1 & 160 & 10 & 33 \\ \text { 97N63W 2CAA } & 01-18-77 & 52 & 1028 & 1028 & -- & 587 & 7.8 & 280 & 81 & 19 \\ \text { 97N63W 3DCCC } & 06-13-67 & 23 & 1028 & 46002 & 16.0 & 1,180 & 7.7 & 500 & 120 & 48 \\ \text { 97N63W 3DDDD } & 11-21-72 & 21 & 1028 & 1028 & -- & 1,010 & 7.4 & 450 & 150 & 18 \\ \text {--do-- } & 08-22-79 & 21 & 1028 & 1028 & 21.0 & 890 & -- & 430 & 100 & 43 \\ & & & & & & & & & & \\ \text { 97N63W11AAAC } & 06-14-67 & 27 & 1028 & 46002 & 10.0 & 1,970 & 7.8 & 1,100 & 340 & 65 \\ \text { 97N63W11AAAC3 } & 10-17-67 & 20 & 1028 & 46002 & 13.5 & 1,510 & 7.7 & 810 & 200 & 76 \\ \text { 97N63W11ACDC } & 11-05-74 & 33 & 1028 & 1028 & -- & 699 & 7.1 & 390 & 100 & 33 \\ \text {--do-- } & 10-16-80 & 33 & 1028 & 1028 & -- & 1,340 & 7.5 & 550 & 150 & 45 \\ \text { 97N63W11D } & 02-18-82 & 49 & 1028 & 1028 & -- & 2,900 & 7.8 & 1,500 & 400 & 120\end{array}$




\begin{tabular}{|c|c|c|c|c|c|c|c|c|c|c|c|c|}
\hline $\begin{array}{c}\text { Sodlum, } \\
\text { dia- } \\
\text { solved } \\
\text { (mg/l } \\
\text { as } \mathrm{Na}) \\
(00930)\end{array}$ & $\begin{array}{l}\text { Potas- } \\
\text { slum, } \\
\text { dis- } \\
\text { solved } \\
\text { (mg/L } \\
\text { as K) } \\
(00935)\end{array}$ & $\begin{array}{c}\text { Chloride, } \\
\text { dis- } \\
\text { solved } \\
\text { (mg/L } \\
\text { as Cl) } \\
(00940)\end{array}$ & $\begin{array}{c}\text { Sulfate, } \\
\text { dis- } \\
\text { solved } \\
(\mathrm{mg} / \mathrm{L} \\
\left.\text { as } \mathrm{SO}_{4}\right) \\
(00945)\end{array}$ & $\begin{array}{c}\text { Fluorlde, } \\
\text { dls- } \\
\text { solved } \\
\text { (mg/L } \\
\text { as F) } \\
\text { (00950) }\end{array}$ & $\begin{array}{c}\text { Silica, } \\
\text { dis- } \\
\text { solved } \\
\left(\mathrm{mg}^{2} \mathrm{~L} \text { as }\right. \\
\left.\mathrm{SiO}_{2}\right) \\
(00955)\end{array}$ & $\begin{array}{c}\text { Bicar- } \\
\text { bonata } \\
\text { FET- } \\
\text { FLD } \\
\left(\mathrm{mg}^{2} \text { as }\right. \\
\left.\mathrm{HCO}_{3}\right) \\
(00440)\end{array}$ & $\begin{array}{c}\text { Carbo- } \\
\text { nate } \\
\text { FET- } \\
\text { FLD } \\
\left(\mathrm{mg}^{2} \text { as }\right. \\
\left.\mathrm{CO}_{3}\right) \\
(00445)\end{array}$ & $\begin{array}{c}\text { Nitro- } \\
\text { gen, } \\
\text { nitrate } \\
\text { total } \\
\text { (mg/L } \\
\text { as N) } \\
(00620)\end{array}$ & $\begin{array}{c}\text { Solids, } \\
\text { sum of } \\
\text { consti- } \\
\text { tuents, } \\
\text { dis- } \\
\text { solved } \\
\text { (mg/L) } \\
\text { (70301) }\end{array}$ & $\begin{array}{c}\text { Iron, } \\
\text { total } \\
\text { recover- } \\
\text { able } \\
(\mu \mathrm{g} / \mathrm{L} \\
\text { as Fe) } \\
(01045)\end{array}$ & $\begin{array}{c}\text { Manga- } \\
\text { nese, } \\
\text { total } \\
\text { recover- } \\
\text { able } \\
(\mu g / L \\
\text { as Mn) } \\
(01055)\end{array}$ & $\begin{array}{c}\text { Boron, } \\
\text { dia- } \\
\text { solved } \\
(\mu \mathrm{g} / \mathrm{L} \\
\text { as B) } \\
(01020)\end{array}$ \\
\hline
\end{tabular}

$\begin{array}{rrrrrrrrrrrrr}380 & 21 & 170 & 650 & -- & -- & 190 & -- & - & 1,410 & -- & -- & 1,700 \\ 130 & 37 & 170 & 1,300 & -- & -- & 110 & -- & 1.40 & 2,190 & 620 & -- & 630 \\ 390 & 23 & 30 & 1,500 & -- & -- & 420 & -- & - & 2,520 & -- & -. & -- \\ 390 & 23 & 30 & 1,500 & -- & -- & -- & -- & \text { ND } & 2,550 & 3,000 & -- & -- \\ 400 & 29 & 23 & 1,800 & -- & -- & -- & -- & .170 & 3,070 & 40 & -- & 1,300 \\ 420 & -- & 12 & 1,800 & 0.32 & -- & - & -- & <.100 & -. & 450 & 450 & --\end{array}$

$\begin{array}{rrrrrrrrrrrrr}120 & 22 & 10 & 420 & -- & -- & 160 & -- & 1.65 & 753 & 3,300 & -- & 800 \\ 220 & 26 & 17 & 1,300 & -- & -- & 270 & -- & -- & 2,020 & -- & -- & -- \\ 150 & 12 & 4.0 & 260 & -- & -- & 500 & -- & \text { ND } & 810 & 100 & -- & 8,800 \\ 150 & -- & 8.0 & 170 & -35 & -- & -- & -- & <.500 & -- & <50 & 600 & -- \\ 320 & 28 & 16 & -- & -- & -- & -- & -- & -- & -- & 90 & -- & -- \\ 320 & 28 & 16 & 1,100 & -- & -- & 410 & -- & -- & 2,060 & - & -- & -- \\ 180 & 21 & 19 & 660 & -- & -- & 210 & 5 & 1.52 & 1,150 & 140 & -- & 860\end{array}$

\begin{tabular}{|c|c|c|c|c|c|c|c|c|c|c|c|c|}
\hline 280 & 19 & 220 & 800 & .50 & 22 & 280 & 0 & -- & 1,730 & -- & -. & 1,100 \\
\hline 280 & 19 & 220 & 800 & .50 & 22 & 280 & $\mathrm{ND}$ & -- & 1,720 & 530 & -- & 1,100 \\
\hline 290 & 18 & 240 & 700 & -- & -- & 290 & -- & -- & 1,640 & -. & -- & . \\
\hline 280 & 21 & 210 & 910 & -- & -- & -. & -- & ND & 1,580 & -- & -- & 1,400 \\
\hline-- & -- & -- & -- & -- & -- & - & -- & -- & - & -- & -- & -- \\
\hline 72 & 9.4 & 15 & 540 & -- & $\ldots$ & 380 & -- & -- & 1,100 & -- & -- & 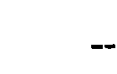 \\
\hline 18 & 100 & 8.0 & 80 & -- & -- & 370 & - & - & 485 & -- & -- & -- \\
\hline 120 & 8.0 & 10 & 350 & -- & -- & 340 & -- & - & 786 & -- & -- & " \\
\hline 20 & 6.0 & 4.0 & 150 & -- & -- & 290 & -- & -- & 445 & $\cdots$ & -- & -- \\
\hline 63 & 10 & 5.0 & 180 & -- & -- & 450 & - & -- & 632 & -- & -- & $=$ \\
\hline 19 & 8.0 & 4.0 & 100 & -- & -- & 350 & -- & -- & 351 & -- & - & -- \\
\hline 17 & 8.0 & 6.0 & 130 & -- & -- & 230 & -- & -- & 372 & -- & -- & -- \\
\hline 84 & 8.7 & 10 & 300 & -- & -- & 350 & -- & -- & 743 & -- & -- & -- \\
\hline 30 & 8.5 & 3.5 & 190 & -- & -- & - & -- & .560 & 587 & 110 & -- & 100 \\
\hline 27 & -- & 7.0 & 150 & .25 & - & - & -- & -. & -- & 250 & 750 & -- \\
\hline 66 & 9.4 & 18 & 860 & -- & -- & 330 & -- & -- & 1,520 & -- & -- & -- \\
\hline 46 & 8.6 & 9.8 & 680 & - & -- & 180 & -- & -- & 1,110 & -- & -- & -- \\
\hline 19 & 8.0 & 4.0 & 100 & -- & $\cdots$ & 350 & -- & -- & 441 & - & - & -- \\
\hline 73 & 6.0 & 10 & 290 & -- & - & 360 & -- & -- & 749 & -- & -- & -- \\
\hline 210 & 49 & 10 & 1,800 & -- & -- & 100 & ND & -- & 2,670 & -- & -- & 1,200 \\
\hline
\end{tabular}


Table 3. Chemical analyses of ground water in Charles Mix and Douglas Counties--Continued

\begin{tabular}{|c|c|c|c|c|c|c|c|c|c|c|}
\hline $\begin{array}{l}\text { Principal aquifer/ } \\
\text { local identifier }\end{array}$ & $\begin{array}{l}\text { Date of } \\
\text { sempie }\end{array}$ & $\begin{array}{c}\text { Depth } \\
\text { of } \\
\text { weil } \\
\text { (feet) } \\
(00003)\end{array}$ & $\begin{array}{c}\text { Agency } \\
\text { collecting } \\
\text { sample } \\
\text { (code } \\
\text { number) } \\
\text { (00027) }\end{array}$ & $\begin{array}{l}\text { Agency } \\
\text { analyzing } \\
\text { sample } \\
\text { (code } \\
\text { number) } \\
\text { (00028) }\end{array}$ & $\begin{array}{c}\text { Water } \\
\text { temper- } \\
\text { eture } \\
\left({ }^{\circ} \mathrm{C}\right) \\
(00010)\end{array}$ & $\begin{array}{c}\text { Specific } \\
\text { conduct- } \\
\text { ence } \\
(\mu \mathrm{S} / \mathrm{cm}) \\
(00095)\end{array}$ & $\begin{array}{c}\text { pH } \\
\text { (standard } \\
\text { units) } \\
(\mathbf{0 0 4 0 0 )}\end{array}$ & $\begin{array}{c}\text { Hardness } \\
\text { totai } \\
(\mathrm{mg} / \mathrm{L} \text { as } \\
\left.\mathrm{CaCO}_{3}\right) \\
(0090)\end{array}$ & $\begin{array}{c}\text { Calcium, } \\
\text { dissolved } \\
\text { (mg/l } \\
\text { as Ce) } \\
(00915)\end{array}$ & $\begin{array}{l}\text { Magne- } \\
\text { aium, } \\
\text { dissolved } \\
\text { (mg/L } \\
\text { as Mg) } \\
(00925)\end{array}$ \\
\hline
\end{tabular}

Delmont aquifer (Cont.)

\begin{tabular}{|c|c|c|c|c|c|c|c|c|c|c|}
\hline 97N63W28BAAA & 05- -69 & 35 & 1028 & 1028 & -. & 1,800 & 8.4 & 1,100 & 240 & 110 \\
\hline --do-- & $06-13-74$ & 35 & 1028 & 1028 & -- & 1,820 & 7.7 & 800 & 180 & 84 \\
\hline 98N62W19ABBB & $04-25-75$ & 55 & 1028 & 1028 & -- & 838 & 7.4 & $280^{\circ}$ & 13 & 60 \\
\hline 98N62W20BBBB & $11-21-72$ & 19 & 1028 & 1028 & -- & 571 & 7.5 & 260 & 98 & 4.0 \\
\hline --do-- & $08-23-79$ & 19 & 1028 & 1028 & 21.0 & 650 & -- & 310 & 80 & 27 \\
\hline $98 \mathrm{~N} 62 \mathrm{~W} 30 \mathrm{BBB}$ & $04-25-75$ & 60 & 1028 & 1028 & -- & 1,080 & 7.6 & 600 & 59 & 110 \\
\hline $98 \mathrm{~N} 62 \mathrm{~W} 31 \mathrm{BCC}$ & $12-09-74$ & 40 & 1028 & 1028 & -- & 884 & 7.3 & 560 & 140 & 51 \\
\hline 98N62W31CDA & $05-01-73$ & 46 & 1028 & 1028 & -. & 2,130 & 7.0 & 730 & 250 & 26 \\
\hline 98N62W31DDBA & $08-06-76$ & 79 & 1028 & 1028 & -- & 2,110 & 7.2 & 430 & 110 & 36 \\
\hline 98N63W27DDBB & $12-09-74$ & 20 & 1028 & 1028 & -- & 1,090 & 7.2 & 600 & 150 & 55 \\
\hline 98N63W36AABA & $12-18-58$ & 37 & 1028 & 1028 & -- & 870 & 7.2 & 410 & 100 & 38 \\
\hline - do-. & $06-29-67$ & 37 & 1028 & 46002 & 13.5 & 755 & 7.8 & 360 & 96 & 28 \\
\hline --do-- & $05-\quad-69$ & 37 & 1028 & 1028 & -- & 880 & 7.4 & 500 & 120 & 50 \\
\hline --do-- & $06-13-74$ & 37 & 1028 & 1028 & -- & 951 & 7.5 & 570 & 150 & 48 \\
\hline --do-- & $08-24-79$ & 37 & 1028 & 1028 & 21.0 & 785 & - & 580 & 140 & 54 \\
\hline --do-- & $05-15-80$ & 37 & 1028 & 1028 & -- & 850 & 7.8 & 350 & 72 & 41 \\
\hline 98N63W36ADBB & $04-11-80$ & 60 & 1028 & 1028 & -- & 837 & 7.5 & 460 & 120 & 38 \\
\hline 98N63W36BDDD & $04-11-80$ & 72 & 1028 & 1028 & -- & 731 & 7.5 & 340 & 92 & 26 \\
\hline 98N63W36CBDD & $04-11-80$ & 50 & 1028 & 1028 & -- & 910 & 7.5 & 350 & 88 & 31 \\
\hline 98N63W36DAAD & $01-19-81$ & 40 & 1028 & 1028 & -- & 733 & 8.0 & 370 & 88 & 36 \\
\hline 99N63W28DDDD & $05-15-80$ & 28 & 1028 & 1028 & -- & 1,800 & 7.7 & 780 & 180 & 79 \\
\hline \multicolumn{11}{|l|}{ Geddes aquifer } \\
\hline 97N66W 5DDDD & $08-10-78$ & 225 & 46002 & 46002 & -- & 2,580 & 7.8 & 560 & 130 & 55 \\
\hline --do-- & 08-20-79 & 225 & 1028 & 1028 & 22.0 & 2,450 & -- & 500 & 130 & 40 \\
\hline 97N66W16ADDB & $11-29-76$ & 219 & 1028 & 1028 & - & 2,570 & 8.1 & 520 & 160 & 32 \\
\hline $97 \mathrm{~N} 66 \mathrm{~W} 23 \mathrm{BCCB}$ & --65 & 280 & 46008 & 46008 & -- & -- & 8.1 & 330 & 97 & 21 \\
\hline 97N66W23DDAA & $06-\quad-65$ & 320 & 46008 & 46008 & -- & -- & 8.1 & 620 & 170 & 46 \\
\hline 100N68W21DDDD & $10-01-69$ & 260 & 1028 & 46002 & 12.0 & 3,840 & 7.7 & 1,000 & 230 & 110 \\
\hline \multicolumn{11}{|l|}{ Greenwood aquifer } \\
\hline $93 \mathrm{~N} 62 \mathrm{~W} 20 \mathrm{~A}$ & $-\quad-68$ & 17 & 1028 & 1028 & -- & 714 & 7.9 & 330 & 110 & 13 \\
\hline 93N62W20ABB & $08-15-59$ & 28 & 1028 & 46002 & 10.0 & 880 & 8.2 & 340 & 88 & 30 \\
\hline --do-- & $07-02-68$ & 28 & 1028 & 46002 & 12.0 & 715 & 7.9 & 330 & 110 & 13 \\
\hline 93N63W 9ABDD & $10-16-79$ & 16 & 46003 & 84610 & 15.0 & 1,340 & 6.9 & 650 & 150 & 66 \\
\hline 93N63W 9BBBB & $07-02-68$ & 32 & 1028 & 46002 & 12.0 & 2,890 & 7.7 & 1,200 & 330 & 82 \\
\hline
\end{tabular}




\begin{tabular}{|c|c|c|c|c|c|c|c|c|c|c|c|c|}
\hline $\begin{array}{c}\text { Sodium, } \\
\text { dis- } \\
\text { solved } \\
\text { (mg/L } \\
\text { as } \mathrm{Na} \text { ) } \\
(00930)\end{array}$ & $\begin{array}{l}\text { Potas- } \\
\text { sium, } \\
\text { dis- } \\
\text { solved } \\
\text { (mg/L } \\
\text { as K) } \\
(00935)\end{array}$ & $\begin{array}{c}\text { Chloride, } \\
\text { dis- } \\
\text { solved } \\
\text { (mg/l } \\
\text { as Cl) } \\
(00940)\end{array}$ & $\begin{array}{c}\text { Sulfate, } \\
\text { dis- } \\
\text { solved } \\
(\mathrm{mg} / \mathrm{L} \\
\left.\text { as } \mathrm{SO}_{4}\right) \\
(00945)\end{array}$ & $\begin{array}{c}\text { Fluoride, } \\
\text { dis- } \\
\text { solved } \\
\text { (mg/L } \\
\text { as F) } \\
(00950)\end{array}$ & $\begin{array}{c}\text { Silica, } \\
\text { dis- } \\
\text { solved } \\
\left(\mathrm{mg}^{2} \text { es }\right. \\
\left.\mathrm{SiO}_{2}\right) \\
(00955)\end{array}$ & $\begin{array}{c}\text { Bicar- } \\
\text { bonate } \\
\text { FET- } \\
\text { FLD } \\
\left(\mathrm{mg}^{2} \mathrm{~L} \text { as }\right. \\
\left.\mathrm{HCO}_{3}\right) \\
(00440)\end{array}$ & $\begin{array}{c}\text { Carbo- } \\
\text { nate } \\
\text { FET- } \\
\text { FLD } \\
\left(\mathrm{mg}^{2} \mathrm{Les}\right. \\
\left.\mathrm{CO}_{3}\right) \\
(00445)\end{array}$ & $\begin{array}{c}\text { Nitro- } \\
\text { gen, } \\
\text { nitrate } \\
\text { total } \\
\text { (mg/L } \\
\text { as N) } \\
(00620)\end{array}$ & $\begin{array}{c}\text { Solids, } \\
\text { sum of } \\
\text { consti- } \\
\text { tuents, } \\
\text { dis- } \\
\text { solved } \\
\text { (mg/l) } \\
(70301)\end{array}$ & $\begin{array}{c}\text { Iron, } \\
\text { total } \\
\text { recover- } \\
\text { able } \\
(\mu \mathrm{g} / \mathrm{L} \\
\text { as Fe) } \\
(01045)\end{array}$ & $\begin{array}{c}\text { Manga- } \\
\text { nese, } \\
\text { total } \\
\text { recover- } \\
\text { able } \\
(\mu g / \mathrm{L} \\
\text { as Mn) } \\
(01055)\end{array}$ & $\begin{array}{c}\text { Boron, } \\
\text { dis- } \\
\text { solved } \\
(\mu \mathrm{g} / \mathrm{L} \\
\text { as B) } \\
(01020)\end{array}$ \\
\hline
\end{tabular}

\begin{tabular}{|c|c|c|c|c|c|c|c|c|c|c|c|c|}
\hline 130 & 11 & 77 & 690 & -- & -- & -- & -. & 0.110 & 1,390 & 100 & -- & 850 \\
\hline 150 & 18 & 75 & 710 & -- & -- & 280 & -- & .060 & 1,350 & 110 & -- & 600 \\
\hline 16 & 8.0 & 15 & 210 & -- & -- & 480 & -- & -- & 557 & -- & -- & -- \\
\hline 4.2 & 4.2 & 4.0 & 64 & -- & -- & -- & -- & .090 & 308 & 140 & -. & ND \\
\hline 5.0 & -- & 5.0 & 110 & 0.18 & -- & -- & -- & .500 & -. & $<50$ & 80 & -- \\
\hline 51 & 40 & 26 & 130 & -- & -- & 460 & -- & -- & 642 & -- & -- & $-\infty$ \\
\hline 16 & 6.0 & 6.0 & 210 & .. & -- & 340 & -. & -- & 597 & -- & -- & -- \\
\hline 220 & 28 & 8.0 & 720 & -- & -- & 620 & -- & -- & 1,550 & -- & -- & - \\
\hline 290 & 28 & 150 & 580 & -. & -- & 410 & -- & -- & 1,390 & -- & -- & -- \\
\hline 54 & 14 & 2.0 & 250 & - & -- & 410 & $-\cdot$ & -. & 729 & -- & -- & -- \\
\hline 36 & 7.2 & 9.2 & 210 & .40 & 26 & 330 & 0 & -- & 588 & -- & -- & 70 \\
\hline 15 & 5.8 & 20 & 190 & -- & -- & 180 & -- & -- & 446 & -- & -- & -- \\
\hline 13 & 4.8 & 7.5 & 320 & -- & -- & -- & -- & .090 & 660 & 2,000 & -- & 100 \\
\hline 18 & 7.0 & 11 & 260 & -- & -- & 370 & -- & .010 & 672 & 130 & -- & 100 \\
\hline 18 & -- & 11 & 270 & 33 & -- & -- & -- & $<.100$ & -- & $<50$ & 700 & -- \\
\hline 15 & 5.0 & 16 & 260 & -- & - & 140 & -- & .400 & 481 & 300 & -- & 160 \\
\hline 18 & 4.0 & 10 & 220 & -- & -- & 280 & -- & -- & 546 & - & -- & -- \\
\hline 41 & 4.0 & 7.0 & 110 & -- & -- & 340 & -- & -. & 446 & - & -- & - \\
\hline 73 & 8.0 & 6.0 & 160 & -. & -- & 400 & -- & -- & 565 & - & -- & -- \\
\hline 14 & 4.0 & 13 & 140 & -- & -- & 310 & - & - & 444 & $\cdots$ & -- & -- \\
\hline 100 & 15 & 4.0 & 920 & - & -- & 110 & -- & .320 & 1,360 & 1,300 & -- & 280 \\
\hline 350 & 55 & 120 & 880 & -- & -- & 340 & -- & 2.35 & 1,770 & 170 & -- & 4,600 \\
\hline 410 & -- & 110 & 860 & .76 & - & -- & -- & -- & -- & $<50$ & 2,100 & -- \\
\hline 410 & 28 & 24 & 1,100 & -- & -- & 290 & -- & -- & 1,880 & $\cdots$ & -- & - \\
\hline-- & -- & 120 & 800 & -- & - & -- & -- & - & 1,680 & - & -- & - \\
\hline- & -- & 210 & 1,600 & -- & $\cdots$ & -- & -- & -- & -- & - & 1,200 & - \\
\hline 570 & 31 & 330 & 1,400 & -- & $\ldots$ & 86 & - & - & 2,710 & - & -- & -- \\
\hline 18 & 6.0 & 4.0 & -- & - & -- & - & -- & -- & -- & 710 & -- & -- \\
\hline 110 & 3.6 & 7.0 & 230 & -- & -- & 280 & -- & -- & 604 & - & -- & - \\
\hline 18 & 6.4 & 4.0 & 96 & -- & -- & 300 & -- & -- & 393 & - & - & $\cdots$ \\
\hline 59 & -. & 38 & 320 & -- & 7.6 & 510 & 0 & -- & -- & 10 & 2 & -- \\
\hline 260 & 14 & 150 & 1,200 & -- & -- & 330 & -- & -- & 2,200 & - & $-\cdot$ & -- \\
\hline
\end{tabular}


Table 3. Chemical analyses of ground water in Charles Mix and Douglas Counties--Continued

\begin{tabular}{|c|c|c|c|c|c|c|c|c|c|c|}
\hline $\begin{array}{l}\text { Principal aquifer/ } \\
\text { local identifier }\end{array}$ & $\begin{array}{l}\text { Date of } \\
\text { sample }\end{array}$ & $\begin{array}{c}\text { Depth } \\
\text { of } \\
\text { well } \\
\text { (feet) } \\
(00003) \\
\end{array}$ & $\begin{array}{c}\text { Agency } \\
\text { collecting } \\
\text { semple } \\
\text { (code } \\
\text { number) } \\
\text { (00027) } \\
\end{array}$ & $\begin{array}{c}\text { Agency } \\
\text { analyzing } \\
\text { sample } \\
\text { (code } \\
\text { number) } \\
\text { (00028) }\end{array}$ & $\begin{array}{c}\text { Weter } \\
\text { temper- } \\
\text { ature } \\
\left({ }^{\circ} \mathrm{C}\right) \\
(00010) \\
\end{array}$ & $\begin{array}{c}\text { Specific } \\
\text { conduct- } \\
\text { ance } \\
(\mu \mathrm{S} / \mathrm{cm}) \\
(00095) \\
\end{array}$ & $\begin{array}{c}\mathrm{pH} \\
\text { (standard } \\
\text { units) } \\
(\mathbf{0 0 4 0 0 )} \\
\end{array}$ & $\begin{array}{c}\text { Hardness } \\
\text { total } \\
\left(\mathrm{mgg}^{\mathrm{L}} \text { as }\right. \\
\left.\mathrm{CaCO}_{3}\right) \\
(00900) \\
\end{array}$ & $\begin{array}{c}\text { Calcium, } \\
\text { dissolved } \\
\text { (mg/L } \\
\text { as Ce) } \\
(00915) \\
\end{array}$ & $\begin{array}{c}\text { Megne- } \\
\text { aium, } \\
\text { dissolve } \\
(\mathrm{mgh} / \\
\text { as } \mathrm{Mg}) \\
(00925) \\
\end{array}$ \\
\hline \multicolumn{11}{|c|}{ Greenwood aquifer (Cont.) } \\
\hline 93N63W13ABBD & $04-27-81$ & 90 & 1028 & 1028 & -- & 1,900 & 6.8 & 1,100 & 320 & 72 \\
\hline \multicolumn{11}{|l|}{ Intermediate aquifer } \\
\hline 96N62W21AACD & $11-01-68$ & 115 & 46002 & 46002 & 11.0 & 3,230 & 7.8 & 1,100 & 400 & 20 \\
\hline 96N63W 3ACAA & $05-03-57$ & 156 & 1028 & 46001 & - & 1,600 & 8.0 & 320 & 90 & 22 \\
\hline 96N63W 6DDCC & $08-01-68$ & 221 & 1028 & 46002 & 11.0 & 2,120 & 8.0 & 300 & 100 & 13 \\
\hline --do-- & $05-\quad-69$ & 221 & 1028 & 1028 & - & 2,170 & 8.5 & 410 & 120 & 27 \\
\hline --do-- & $06-13-74$ & 221 & 1028 & 1028 & -- & 2,130 & 7.6 & 400 & 120 & 24 \\
\hline --do-- & $08-21-79$ & 221 & 1028 & 1028 & 25.0 & 1,980 & -- & 320 & 90 & 22 \\
\hline 96N63W 8AAC & $01-27-75$ & 80 & 1028 & 1028 & -- & 2,020 & 7.3 & 370 & 110 & 23 \\
\hline --do-- & $02-19-75$ & 80 & 1028 & 1028 & -- & 2,220 & 7.3 & 1,000 & 290 & 79 \\
\hline 96N63W 9DAAA & $07-10-73$ & 165 & 1028 & 1028 & -- & 3,680 & 6.9 & 1,000 & 370 & 26 \\
\hline --do-- & $08-24-79$ & 165 & 1028 & 1028 & 21.0 & 2,500 & -- & 1,100 & 320 & 78 \\
\hline 96N63W15BBBB & $12-19-60$ & 155 & 46008 & 46008 & -- & 2,000 & -- & 420 & 120 & 28 \\
\hline $96 \mathrm{~N} 63 \mathrm{~W} 15 \mathrm{CCAA}$ & $09-15-58$ & 172 & 1028 & 46001 & -- & 2,000 & 7.3 & 470 & 140 & 30 \\
\hline --do-- & $08-01-67$ & 172 & 1028 & 1028 & -- & 1,680 & 7.3 & 450 & 140 & 25 \\
\hline --do-- & $08-01-68$ & 172 & 1028 & 46002 & 13.0 & 1,720 & 7.9 & 430 & 130 & 26 \\
\hline 96N63W21CCCC & $08-29-78$ & 62 & 46007 & 46002 & -- & 2,030 & 7.8 & 880 & 250 & 61 \\
\hline --do-- & $08-22-79$ & 62 & 1028 & 1028 & 21.0 & 3,600 & -. & 2,400 & 540 & 260 \\
\hline 96N63W22BBBB & $02-07-57$ & 176 & 1028 & 46001 & -- & 1,800 & 7.7 & 370 & 110 & 24 \\
\hline 96N63W35BBCB & $12-19-60$ & 120 & 46008 & 46008 & -- & 2,200 & -- & 340 & 92 & 26 \\
\hline 96N64W 1AAAA & $10-15-69$ & 152 & 1028 & 1028 & -- & 3,520 & 8.1 & 980 & 280 & 69 \\
\hline $96 \mathrm{~N} 64 \mathrm{~W} 1 \mathrm{BCCC}$ & $04-07-78$ & 155 & 1028 & 46002 & -- & 4,810 & 8.1 & 2,300 & 530 & 230 \\
\hline 96N64W 1BCCC & $08-21-79$ & 155 & 1028 & 1028 & 21.0 & 4,700 & -- & 2,300 & 440 & 300 \\
\hline 96N64W12BAAA & $01-21-57$ & 189 & 1028 & 46001 & - & 1,900 & 7.6 & 310 & 83 & 25 \\
\hline 97N63W13DDDB & $04-19-77$ & 180 & 1028 & 1028 & -- & 990 & 7.5 & 520 & 130 & 47 \\
\hline 97N63W21BBBB2 & $08-21-79$ & 124 & 1028 & 1028 & 22.0 & 3,250 & - & 1,900 & 510 & 150 \\
\hline 97N63W25CBBB & $09-01-60$ & 196 & 1028 & 46004 & -- & -- & - & 250 & 68 & 20 \\
\hline 97N63W34DCBB & $-\quad-57$ & 157 & 1028 & 46001 & -- & 1,900 & 7.7 & 410 & 120 & 26 \\
\hline --do-- & $08-01-67$ & 157 & 1028 & 46002 & -- & 1,930 & 7.3 & 340 & 100 & 23 \\
\hline $97 N 64 \mathrm{~W} 17 \mathrm{BBBB}$ & --65 & 110 & 46008 & 46008 & -- & $\ldots$ & 7.4 & 400 & 120 & 25 \\
\hline 97N64W27AAAA & --65 & 210 & 46008 & 46008 & -- & -- & 7.9 & 490 & 120 & 45 \\
\hline 97N64W36DADD & $12-19-60$ & 192 & 46008 & 46008 & 12.0 & 2,150 & -- & 320 & 88 & 24 \\
\hline $\mathrm{N} 63 \mathrm{~W} 36 \mathrm{C}$ & $11-21-72$ & 43 & 1028 & 1028 & -- & 850 & 7.6 & 370 & 110 & 23 \\
\hline --do-- & 08-23-79 & 43 & 1028 & 1028 & 21.0 & 680 & -- & 350 & 87 & 33 \\
\hline
\end{tabular}




\begin{tabular}{|c|c|c|c|c|c|c|c|c|c|c|c|c|}
\hline $\begin{array}{c}\text { Sodium, } \\
\text { dis- } \\
\text { solved } \\
\text { (mg/L } \\
\text { as } \mathrm{Na}) \\
(00930)\end{array}$ & $\begin{array}{l}\text { Potas- } \\
\text { slum, } \\
\text { dis- } \\
\text { solvad } \\
\text { (mg/L } \\
\text { as K) } \\
(00935)\end{array}$ & $\begin{array}{c}\text { Chlorida, } \\
\text { dis- } \\
\text { solved } \\
\text { (mg/L } \\
\text { as Cl) } \\
(00940)\end{array}$ & $\begin{array}{c}\text { Sulfate, } \\
\text { dis- } \\
\text { solved } \\
(\mathrm{mg} / \mathrm{L} \\
\left.\text { as } \mathrm{SO}_{4}\right) \\
(00945)\end{array}$ & $\begin{array}{c}\text { Fluoride, } \\
\text { dis- } \\
\text { soived } \\
\text { (mg/L } \\
\text { as F) } \\
(00950)\end{array}$ & $\begin{array}{c}\text { Silica, } \\
\text { dis- } \\
\text { solved } \\
\left(\mathrm{mg}^{2} \mathrm{~L} \text { as }\right. \\
\left.\mathrm{SiO}_{2}\right) \\
(00955)\end{array}$ & $\begin{array}{c}\text { Bicar- } \\
\text { bonate } \\
\text { FET- } \\
\text { FLD } \\
\left(\mathrm{mg}^{2} \mathrm{~L} \text { as }\right. \\
\left.\mathrm{HCO}_{3}\right) \\
(00440)\end{array}$ & $\begin{array}{c}\text { Carbo- } \\
\text { nate } \\
\text { FET- } \\
\text { FLD } \\
(\mathrm{mg} / \mathrm{L} \text { as } \\
\left.\mathrm{CO}_{3}\right) \\
(00445)\end{array}$ & $\begin{array}{c}\text { Nitro- } \\
\text { gen, } \\
\text { nitrate } \\
\text { total } \\
(\mathrm{mg} / \mathrm{h} \\
\text { as } \mathrm{N} \text { ) } \\
(00620)\end{array}$ & $\begin{array}{l}\text { Solids, } \\
\text { sum of } \\
\text { consti- } \\
\text { tuenta, } \\
\text { dis- } \\
\text { solved } \\
\text { (mg/h) } \\
\text { (70301) }\end{array}$ & $\begin{array}{c}\text { Iron, } \\
\text { total } \\
\text { recover- } \\
\text { able } \\
(\mu g / L \\
\text { as Fe) } \\
(01045)\end{array}$ & $\begin{array}{l}\text { Manga- } \\
\text { nese, } \\
\text { total } \\
\text { racover- } \\
\text { abie } \\
(\mu g / 1 \\
\text { as Mn) } \\
(01055)\end{array}$ & $\begin{array}{c}\text { Boron, } \\
\text { dis. } \\
\text { solved } \\
(\mu g / L \\
\text { as B) } \\
(01020)\end{array}$ \\
\hline
\end{tabular}

$\begin{array}{rrrrrrrrrr}73 & 16 & 26 & 810 & - & \ldots & 520 & \ldots & \ldots & 1,580\end{array}$

\begin{tabular}{|c|c|c|c|c|c|c|c|c|c|c|c|c|}
\hline 350 & 60 & 16 & 1,600 & -- & -- & 130 & -. & -- & 2,510 & -- & -- & -- \\
\hline 350 & 29 & 64 & 590 & -- & -. & 470 & 0 & -- & 1,380 & -- & -- & -- \\
\hline 330 & 22 & 170 & 620 & -- & -- & 240 & -- & -- & 1,370 & -- & -. & -- \\
\hline 330 & 19 & 160 & 650 & -- & -- & -- & -- & 1.07 & 1,410 & 520 & $\cdots$ & 2,500 \\
\hline 330 & 29 & 180 & 660 & -- &.- & 250 & -- & .010 & 1,460 & ND & -- & 2,700 \\
\hline 320 & -- & 170 & 480 & 0.44 & - & -- & -- & -- & -. & 50 & 1,500 & -- \\
\hline 360 & 26 & 210 & 440 & -- & -- & 310 & -- & -- & 1,320 & -- & $\cdots$ & -- \\
\hline 220 & 18 & 51 & 870 & -- & -- & 400 & -- & -- & 1,720 & -- & -- & $\ldots$ \\
\hline 450 & 32 & 100 & 1,700 & -- & -- & 290 & -- & ND & 2,820 & 80 & -- & 1,500 \\
\hline 370 & $-\cdot$ & 25 & 1,700 & .33 & -- & -- & -- & -- & -- & 150 & 3,100 & -. \\
\hline 320 & 20 & 280 & 410 & .70 & -- & -- & -- & -- & 1,350 & -. & -- & -. \\
\hline 270 & 25 & 160 & 430 & -- & -- & 410 & 17 & -- & 1,280 & -- & -- & -- \\
\hline 250 & 21 & 120 & 350 & - & -- & 450 & -- & - & 1,130 & -- & -- & -- \\
\hline 270 & 22 & 120 & 460 & -- & -- & 410 & -. & - & 1,230 & -- & -- & 530 \\
\hline 110 & 29 & 170 & 740 & -- & -- & 210 & -- & 1.75 & 1,460 & 500 & -- & 1,300 \\
\hline 180 & -- & 150 & 2,200 & .36 & -. & -. & -- & -. & -. & 2,400 & 2,200 & -- \\
\hline 290 & 29 & 180 & 510 & 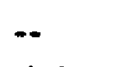 & -- & 340 & 0 & -- & 1,310 & -- & -. & -- \\
\hline 350 & 15 & 210 & 520 & 1.1 & -- & -- & -- & -- & 1,360 & -- & -- & -- \\
\hline 520 & 33 & 80 & 1,600 & -- & -- & -- & -- & ND & 2,730 & 50 & -- & 3,400 \\
\hline 430 & 48 & 24 & 2,800 & - & -- & 160 & -- & 4.40 & 4,180 & 210 & -- & 4,300 \\
\hline 520 & -- & 16 & 3,000 & .33 & -- & -. & -- & -- & -- & 250 & 2,300 & -- \\
\hline 370 & 25 & 220 & 620 & -- & -- & 240 & 0 & - & 1,460 & -- & - & -- \\
\hline 18 & 4.0 & 10 & 270 & -. & - & 330 & -- & -- & 644 & -- & -- & -- \\
\hline 170 & -- & 88 & 1,800 & .35 & -- & $-\cdot$ & -- & -- & -- & $<50$ & 2,700 & -- \\
\hline 360 & -- & 58 & 640 & -- & - & -- & -- & -- & 1,430 & $\cdots$ & $\cdots$ & $\cdots$ \\
\hline 270 & 21 & 200 & 640 & -- & -- & 170 & 0 & -- & 1,360 & -. & -- & -- \\
\hline 340 & 19 & 50 & 560 & -- & -- & 490 & -- & -- & 1,330 & -- & -- & -- \\
\hline-- & -- & 220 & 740 & -- & - & -- & -- & -- & -- & -- & -- & - \\
\hline 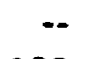 & -- & 280 & 670 & -- & -- & -- & -- & -- & -- & -- & -- & -- \\
\hline 320 & 22 & 140 & 610 & .90 & -- & -- & -- & -- & 1,310 & - & -- & - \\
\hline 19 & 6.5 & 12 & 150 & -- & -- & - & -- & .200 & 447 & 120 & -- & 80 \\
\hline 15 & -- & 8.0 & 90 & .21 & -- & -- & -- & $<.500$ & -- & 200 & 500 & -- \\
\hline
\end{tabular}


Table 3. Chemical analyses of ground water in Charles Mix and Douglas Counties--Continued

\begin{tabular}{|c|c|c|c|c|c|c|c|c|c|c|}
\hline $\begin{array}{l}\text { Princlpal aquifer/ } \\
\text { local identifier }\end{array}$ & $\begin{array}{l}\text { Date of } \\
\text { sample }\end{array}$ & $\begin{array}{l}\text { Depth } \\
\text { of } \\
\text { well } \\
\text { (feet) } \\
(00003)\end{array}$ & $\begin{array}{l}\text { Agency } \\
\text { collecting } \\
\text { sample } \\
\text { (code } \\
\text { number) } \\
\text { (00027) }\end{array}$ & $\begin{array}{l}\text { Agency } \\
\text { analyzing } \\
\text { sample } \\
\text { (code } \\
\text { number) } \\
\text { (00028) }\end{array}$ & $\begin{array}{l}\text { Water } \\
\text { temper- } \\
\text { ature } \\
\left({ }^{\circ} \mathrm{C}\right) \\
(00010)\end{array}$ & $\begin{array}{c}\text { Specific } \\
\text { conduct- } \\
\text { ance } \\
(\mu \mathrm{S} / \mathrm{cm}) \\
(00095)\end{array}$ & $\begin{array}{c}\text { pH } \\
\text { (standard } \\
\text { units) } \\
(\mathbf{0 0 4 0 0 )}\end{array}$ & $\begin{array}{c}\text { Herdneas } \\
\text { total } \\
\left(\mathrm{mgll}^{2} \text { as }\right. \\
\left.\mathrm{CaCO}_{3}\right) \\
(00900)\end{array}$ & $\begin{array}{c}\text { Calcium, } \\
\text { dissolved } \\
\text { (mg/h } \\
\text { as Ca) } \\
(00915)\end{array}$ & $\begin{array}{l}\text { Magne- } \\
\text { sium, } \\
\text { dissolved } \\
(\mathrm{mg} / \mathrm{L} \\
\text { aa Mg) } \\
(00925)\end{array}$ \\
\hline
\end{tabular}

Pleistocene aquifer

\begin{tabular}{|c|c|c|c|c|c|c|c|c|c|c|}
\hline 94N62W26AAAA & $06-15-67$ & 38 & 1028 & 46002 & 12.0 & 1,500 & 6.1 & 620 & 200 & 29 \\
\hline 94N63W18ABBB & $08-29-78$ & 43 & 1028 & 46002 & -- & 2,410 & 7.4 & 1,300 & 370 & 100 \\
\hline 94N63W18BDDB & --76 & 160 & 1028 & 1028 & -- & 4,350 & 7.1 & 2,400 & 440 & 310 \\
\hline --do-- & $11-09-76$ & 160 & 1028 & 1028 & -- & 735 & 8.0 & 240 & 58 & 23 \\
\hline 94N64W 7CBBC & $10-15-79$ & 25 & 46003 & 84610 & 21.0 & 1,590 & 6.9 & -- & -- & 64 \\
\hline 94N64W20CCBB & $07-03-68$ & 21 & 1028 & 46002 & 12.0 & 1,720 & 7.8 & 570 & 150 & 47 \\
\hline 94N64W20DBDC & $10-15-79$ & 20 & 46003 & 84610 & 15.5 & 2,020 & 6.0 & 620 & 150 & 63 \\
\hline 94N65W12DABB & $07-03-68$ & 17 & 1028 & 46002 & 12.0 & 1,720 & 7.8 & 710 & 180 & 64 \\
\hline $95 \mathrm{~N} 62 \mathrm{~W} 21 \mathrm{BBBB}$ & $11-09-69$ & 202 & 1028 & 46002 & 12.0 & 2,700 & 8.1 & 930 & 240 & 80 \\
\hline 96N63W17BBD & $05-13-75$ & 65 & 1028 & 1028 & -- & 1,770 & 6.9 & 900 & 200 & 97 \\
\hline 96N65W 8AABD & $07-13-68$ & 132 & 1028 & 46002 & -- & 1,270 & 7.8 & 480 & 140 & 31 \\
\hline 96N65W10CAAA & --65 & 40 & 46008 & 46008 & -- & -- & 8.4 & 220 & 52 & 22 \\
\hline 96N65W17ABAC & - -68 & 80 & 1028 & 1028 & -- & 1,730 & 7.7 & 880 & 260 & 53 \\
\hline --do-- & $04-03-72$ & 80 & 1028 & 1028 & -- & 1,870 & 7.3 & 830 & 250 & 52 \\
\hline 96N65W17ABCA & $05-14-68$ & 32 & 1028 & 46002 & -- & 1,730 & 7.8 & 870 & 260 & 53 \\
\hline 96N65W19DADC & $06-\quad-65$ & 140 & 1028 & 1028 & -- & -- & 7.3 & 850 & 250 & 56 \\
\hline 96N65W19DDDC & --65 & 140 & 46008 & 46008 & -- & -- & 7.3 & 860 & 250 & 56 \\
\hline 97N63W 1CCCC & $07-01-68$ & 24 & 1028 & 46002 & 11.0 & 2,000 & 7.7 & 1,100 & 280 & 91 \\
\hline --do-- & $08-13-69$ & 24 & 1028 & 46002 & 12.0 & 1,810 & 8.2 & 1,200 & 280 & 120 \\
\hline 97N63W 6AAAA & $06-21-67$ & 72 & 1028 & 46002 & 17.0 & 2,280 & 7.5 & 1,400 & 410 & 100 \\
\hline 97N63W14BBBB & $10-02-69$ & 31 & 1028 & 46002 & 11.0 & 1,170 & 7.9 & 530 & 100 & 69 \\
\hline --do-- & $08-22-79$ & 31 & 1028 & 1028 & 23.0 & 990 & -- & 400 & 110 & 32 \\
\hline 97N63W14BDAA & $10-24-74$ & 30 & 1028 & 1028 & -- & 1,980 & 7.9 & 1,300 & 400 & 82 \\
\hline --do-- & $01-14-75$ & 30 & 1028 & 1028 & -- & 1,260 & 7.4 & 410 & 75 & 54 \\
\hline 97N64W23BBBB & $06-21-67$ & 72 & 1028 & 46002 & 16.0 & 5,050 & 7.4 & 2,600 & 540 & 300 \\
\hline 97N64W35DADD & $09-21-67$ & 238 & 1028 & 1028 & 12.0 & 2,360 & 8.1 & 370 & 130 & 12 \\
\hline 97N66W30BCBB & $07-13-67$ & 22 & 1028 & 46002 & 17.0 & 1,930 & 7.9 & 770 & 210 & 59 \\
\hline $98 \mathrm{~N} 62 \mathrm{~W} 17 \mathrm{CCCC}$ & $07-07-67$ & 27 & 1028 & 46006 & 14.0 & 920 & 7.8 & 480 & 110 & 49 \\
\hline 98N62W19DABB2 & $06-03-57$ & 12 & 1028 & 46002 & -- & 800 & 7.9 & 550 & 120 & 60 \\
\hline 98N63W14BBBB & $07-07-67$ & 33 & 1028 & 46002 & 14.0 & 520 & 8.3 & 170 & 45 & 13 \\
\hline 98N63W22DDDD & $07-31-68$ & 200 & 1028 & 46002 & -- & 2,460 & 7.9 & 170 & 54 & 8.6 \\
\hline 98N63W28DDCC & $09-01-60$ & 180 & 46008 & 46004 & -- & -- & -- & 210 & 64 & 13 \\
\hline 98N66W 8CBDB & $10-11-68$ & 276 & 1028 & 46002 & 21.0 & 2,370 & 7.4 & 1,200 & 340 & 77 \\
\hline 99N63W 5CCCC & $10-28-66$ & 19 & 1028 & 46002 & -- & 3,500 & 7.4 & 1,400 & 410 & 84 \\
\hline 99N63W22BAAA & $07-07-67$ & 27 & 1028 & 46002 & 16.0 & 1,250 & 7.7 & 600 & 140 & 61 \\
\hline
\end{tabular}




\begin{tabular}{|c|c|c|c|c|c|c|c|c|c|c|c|c|}
\hline $\begin{array}{c}\text { Sodium, } \\
\text { dis- } \\
\text { solvad } \\
\text { (mg/L } \\
\text { as } \mathrm{Na} \text { ) } \\
(00930)\end{array}$ & $\begin{array}{l}\text { Potaa- } \\
\text { sium, } \\
\text { dis- } \\
\text { solved } \\
\text { (mg/L } \\
\text { as K) } \\
(00935)\end{array}$ & $\begin{array}{c}\text { Chloride, } \\
\text { dis- } \\
\text { solved } \\
\text { (mgll } \\
\text { as Cl) } \\
\text { (00940) }\end{array}$ & $\begin{array}{c}\text { Sulfate, } \\
\text { dis- } \\
\text { solved } \\
\text { (mg/L } \\
\text { as } \mathrm{SO}_{4} \text { ) } \\
(00945)\end{array}$ & $\begin{array}{c}\text { Fluorlde, } \\
\text { dis- } \\
\text { solved } \\
\text { (mg/L } \\
\text { as F) } \\
\text { (00950) }\end{array}$ & $\begin{array}{c}\text { Silica, } \\
\text { dis- } \\
\text { solved } \\
\left(\mathrm{mg}^{2} \mathrm{~L} \text { as }\right. \\
\left.\mathrm{SiO}_{2}\right) \\
(00955)\end{array}$ & $\begin{array}{c}\text { Bicar- } \\
\text { bonate } \\
\text { FET- } \\
\text { FLD } \\
\text { (mg/l as } \\
\left.\mathrm{HCO}_{3}\right) \\
(00440)\end{array}$ & $\begin{array}{c}\text { Carbo- } \\
\text { nate } \\
\text { FET- } \\
\text { FLD } \\
\text { (mg/L as } \\
\left.\mathrm{CO}_{3}\right) \\
(00445)\end{array}$ & $\begin{array}{c}\text { Nitro- } \\
\text { gen, } \\
\text { nitrate } \\
\text { total } \\
\text { (mg/L } \\
\text { as N) } \\
(00620)\end{array}$ & $\begin{array}{l}\text { Solids, } \\
\text { sum of } \\
\text { consti- } \\
\text { tuents, } \\
\text { dis- } \\
\text { solved } \\
\text { (mgll) } \\
\text { (70301) }\end{array}$ & $\begin{array}{c}\text { Iron, } \\
\text { total } \\
\text { racover- } \\
\text { able } \\
(\mu \mathrm{g} / \mathrm{L} \\
\text { as Fe) } \\
(01045)\end{array}$ & $\begin{array}{c}\text { Manga- } \\
\text { nese, } \\
\text { total } \\
\text { recover- } \\
\text { able } \\
(\mu g / L \\
\text { as Mn) } \\
(01055)\end{array}$ & $\begin{array}{c}\text { Boron, } \\
\text { dis- } \\
\text { solved } \\
\text { ( } \mu g / L \\
\text { as B) } \\
(01020)\end{array}$ \\
\hline
\end{tabular}

\begin{tabular}{|c|c|c|c|c|c|c|c|c|c|c|c|c|}
\hline 64 & 12 & 20 & 910 & -- & -- & 320 & -- & -- & 1,390 & -- & -- & -- \\
\hline 82 & 15 & 140 & 1,100 & -- & -- & 250 & -- & 0.140 & 1,930 & 390 & -- & 170 \\
\hline 260 & 43 & 210 & -- & -- & -- & -. & -- & -- & - & -. & $\cdots$ & -- \\
\hline 68 & 3.0 & 8.0 & 230 & -- & -- & 200 & -- & -- & 484 & -- & -- & -- \\
\hline 47 & -- & 17 & 310 & -- & 9.7 & 460 & 0 & - & -- & 10 & 7 & -- \\
\hline 190 & 8.1 & 49 & 480 & -- & -- & 440 & -- & -- & 1,140 & -- & -- & -- \\
\hline 200 & -- & 150 & 650 & -- & 7.4 & 450 & 0 & -- & -- & 20 & 5,000 & -- \\
\hline 110 & 9.7 & 46 & 640 & -- & -. & 300 & -- & -- & 1,200 & -- & -- & -- \\
\hline 340 & 27 & 62 & 1,000 & -- & -- & 560 & -- & -- & 2,020 & -- & -- & -- \\
\hline 150 & 10 & 20 & 750 & -- & - & 330 & -- & -- & 1,390 & -- & -- & - \\
\hline 77 & 18 & 7.0 & 520 & -- & -- & 160 & -- & -- & 872 & $\cdots$ & -- & 160 \\
\hline- & -- & 960 & .0 & -- & -- & -- & -- & -- & 2,520 & -- & -- & -- \\
\hline 59 & 15 & 11 & -- & -- & -- & -. & -- & -- & -- & -- & -- & -- \\
\hline 50 & 14 & 11 & 560 & -- & -- & -- & -- & .110 & 1,100 & -- & - & -- \\
\hline 59 & 15 & 11 & 810 & - & -- & 300 & -- & - & 1,350 & -- & -- & -- \\
\hline- & -- & 15 & 720 & -- & - & -- & -- & -- & -- & -- & -- & -- \\
\hline-- & -- & 15 & 720 & -. & -- & -- & -- & -- & 1,220 & -- & - & -- \\
\hline 59 & 8.2 & 12 & 1,100 & -- & -- & 260 & -- & -- & 1,680 & -- & -- & -- \\
\hline 110 & 5.5 & 14 & 950 & -- & -- & 230 & -. & -- & 1,590 & -- & -- & 450 \\
\hline 35 & 15 & 26 & 1,000 & -- & -- & 350 & -- & -- & 1,760 & -- & -- & -- \\
\hline 80 & 9.7 & 6.5 & 320 & -- & -- & 370 & -- & -- & 765 & -- & -- & -- \\
\hline 100 & -. & 18 & 180 & .31 & -- & -- & -- & -- & -- & 1,000 & 260 & -- \\
\hline 54 & 13 & 19 & 940 & -- & -- & 390 & -- & - & 1,700 & -- & $\cdots$ & -- \\
\hline 93 & 8.0 & 15 & 350 & -- & -- & 430 & -- & -- & 805 & -- & -- & -- \\
\hline 440 & 54 & 120 & 2,600 & -- & -- & 490 & -- & -- & 4,300 & -- & -- & -- \\
\hline 370 & 24 & 220 & 490 & -- & -- & 260 & -. & -- & 1,380 & -- & -- & -- \\
\hline 98 & 11 & 90 & 710 & -- & -- & 190 & -- & -- & 1,270 & -- & -- & -- \\
\hline 7.0 & 4.9 & 10 & 190 & -- & - & 240 & -- & -- & 491 & -- & -- & -- \\
\hline 47 & 74 & 270 & 62 & -- & -- & 360 & 0 & -- & 812 & -- & -- & -- \\
\hline 46 & 8.6 & 15 & 63 & -- & -- & 190 & -- & -- & 284 & -- & -- & -- \\
\hline 470 & 30 & 280 & 490 & -- & -- & 400 & -- & -. & 1,530 & -- & -- & -- \\
\hline 380 & -- & 110 & 710 & -- & -- & 150 & -- & -- & 1,460 & -- & - & -- \\
\hline 120 & 17 & 180 & 1,000 & -- & -- & 130 & -. & -- & 1,800 & -- & -- & -- \\
\hline 240 & -- & -- & 1,400 & -- &.- & 330 & -- & -- & 3,500 & -- & -- & -- \\
\hline 48 & 7.5 & 20 & 350 & -- & -- & 310 & -- & -- & 779 & -- & -- & - \\
\hline
\end{tabular}


Table 3. Chemical analyses of ground water in Charles Mix and Douglas Counties--Continued

\begin{tabular}{|c|c|c|c|c|c|c|c|c|c|c|}
\hline $\begin{array}{l}\text { Principal aqulfer/ } \\
\text { local identifier }\end{array}$ & $\begin{array}{l}\text { Date of } \\
\text { aample }\end{array}$ & $\begin{array}{c}\text { Depth } \\
\text { of } \\
\text { well } \\
\text { (feet) } \\
(00003)\end{array}$ & $\begin{array}{c}\text { Agency } \\
\text { collecting } \\
\text { sample } \\
\text { (code } \\
\text { number) } \\
\text { (00027) }\end{array}$ & $\begin{array}{c}\text { Agency } \\
\text { analyzing } \\
\text { aample } \\
\text { (code } \\
\text { number) } \\
\text { (00028) }\end{array}$ & $\begin{array}{l}\text { Water } \\
\text { temper- } \\
\text { ature } \\
\left({ }^{\circ} \mathrm{C}\right) \\
(00010)\end{array}$ & $\begin{array}{c}\text { Specific } \\
\text { conduct- } \\
\text { ence } \\
(\mu 5 / \mathrm{cm}) \\
(00095)\end{array}$ & $\begin{array}{c}\mathrm{pH} \\
\text { (atandard } \\
\text { units) } \\
(00400)\end{array}$ & $\begin{array}{c}\text { Hardneaa } \\
\text { total } \\
(\mathrm{mg} / \mathrm{L} \text { aa } \\
\left.\mathrm{CaCO}_{3}\right) \\
(00900)\end{array}$ & $\begin{array}{c}\text { Calcium, } \\
\text { diaaolved } \\
\text { (mg/L } \\
\text { aa Ca) } \\
(00915)\end{array}$ & $\begin{array}{l}\text { Magne- } \\
\text { aium, } \\
\text { dissolved } \\
\text { (mg/h } \\
\text { as Mg) } \\
(00925)\end{array}$ \\
\hline
\end{tabular}

Pleistocene aquifer (Cont.)

\begin{tabular}{|c|c|c|c|c|c|c|c|c|c|c|}
\hline 99N68W24CCB & $08-16-61$ & 25 & 46002 & 46001 & -- & 9,000 & -- & 3,500 & 400 & 610 \\
\hline 99N68W29BBBB & $07-12-67$ & 68 & 1028 & 46002 & 18.0 & 905 & 8.2 & 350 & 99 & 26 \\
\hline $100 \mathrm{~N} 68 \mathrm{~W} 35 \mathrm{CDCC}$ & $07-12-67$ & 33 & 1028 & 46002 & 13.5 & 1,690 & 8.2 & 860 & 290 & \\
\hline 100N69W19AAA & $09-17-68$ & 56 & 1028 & 46002 & 13.0 & 4,690 & 7.6 & 2,300 & 460 & 29 \\
\hline
\end{tabular}

Till aquifer

97N67W 5BCC

\begin{tabular}{|c|c|c|c|c|c|c|c|c|c|c|}
\hline 93N63W 3CBDD2 & $09-04-79$ & 100 & 1028 & 80020 & 15.0 & 2,180 & 8.3 & 58 & 15 & 4.9 \\
\hline $94 \mathrm{~N} 62 \mathrm{~W} 3 \mathrm{CCBB}$ & $10-29-79$ & 318 & 46003 & 84610 & 13.0 & 1,630 & 6.8 & 360 & 99 & 27 \\
\hline $94 \mathrm{~N} 62 \mathrm{~W} 6 \mathrm{BCCC}$ & $10-29-79$ & 400 & 46003 & 84610 & 14.0 & 1,930 & 7.7 & 230 & 58 & 21 \\
\hline 94N62W26BBB & $10-30-79$ & 333 & 46003 & 84610 & 11.5 & 1,440 & 8.2 & 110 & 26 & 11 \\
\hline $94 \mathrm{~N} 62 \mathrm{~W} 34 \mathrm{CDCA}$ & $07-16-69$ & 475 & 1028 & 46002 & 16.0 & 1,950 & 8.6 & 86 & 18 & 9.9 \\
\hline 94N63W 6CCCB & $10-16-79$ & 350 & 46003 & 84610 & 14.5 & 2,410 & 7.4 & 60 & 16 & 4.8 \\
\hline 94N63W 9BABA & $10-16-79$ & 400 & 46003 & 84610 & 15.5 & 2,210 & 7.5 & 46 & 12 & 4.0 \\
\hline 94 N63W27BABB & $10-16-78$ & 407 & 46003 & 84610 & 15.5 & 2,150 & 9.9 & 4 & 1.2 & .20 \\
\hline 94 N63W30CCBB & $10-16-79$ & 344 & 46003 & 84610 & 17.0 & 2,280 & 7.5 & 38 & 9.2 & 3.6 \\
\hline 94N63W33ADDD & $09-12-69$ & 380 & 1028 & 46002 & -- & 3,040 & 8.3 & 54 & 14 & 4.6 \\
\hline 94N64W 5ABA & $09-12-69$ & 365 & 1028 & 46002 & - & 2,260 & 8.4 & 89 & 16 & 12 \\
\hline 94N64W 9AAAB & $10-16-79$ & 321 & 46003 & 84610 & 16.5 & 2,130 & 6.0 & 63 & 15 & 6.3 \\
\hline 94N64W35BBBD & $09-12-69$ & 170 & 1028 & 46002 & 16.0 & 2,080 & 8.4 & 89 & 16 & 12 \\
\hline 95N63W20DDAD & $09-12-69$ & 493 & 1028 & 46002 & 13.0 & 2,450 & 8.1 & 110 & 23 & 12 \\
\hline 95N64W10CDDD & $09-12-69$ & 505 & 1028 & 46002 & -- & 1,880 & 8.3 & 220 & 75 & 8.0 \\
\hline 95N65W 8ABDD & $10-28-48$ & 118 & 1028 & 1028 & 13.5 & -- & 7.9 & 470 & 99 & 55 \\
\hline --do-- & $11-05-48$ & 118 & 1028 & 1028 & 13.0 & -- & 7.8 & 59 & 16 & 4.6 \\
\hline --do-- & $11-05-48$ & 118 & 1028 & 1028 & -- & -- & 7.9 & 61 & 16 & 5.2 \\
\hline 95N65W 9AACB & $09-10-47$ & 380 & 1028 & 810 & 16.0 & -- & 8.0 & 84 & 22 & 7.0 \\
\hline 96N62W34BACD & $12-19-60$ & 450 & 1028 & 46008 & -- & 3,600 & -- & 1,300 & 370 & 96 \\
\hline 96N64W 7AAAA & $12-19-60$ & 370 & 46008 & 46008 & -- & 2,700 & - & 100 & 24 & 10 \\
\hline --do-- & $-\quad-65$ & 370 & 1028 & 1028 & -- & -- & -- & 100 & 24 & 10 \\
\hline 96N64W17AAAD & $-\quad-38$ & 350 & 1028 & 1028 & -- & -- & -- & -- & - & -- \\
\hline --do-- & $03-20-40$ & 350 & 1028 & 1028 & -- & -- & -- & 490 & 130 & 39 \\
\hline --do-- & $10-\quad-53$ & 350 & 1028 & 1028 & -- & -- & 7.6 & 490 & 140 & 35 \\
\hline
\end{tabular}




\begin{tabular}{|c|c|c|c|c|c|c|c|c|c|c|c|c|}
\hline $\begin{array}{c}\text { Sodium, } \\
\text { dis- } \\
\text { solved } \\
(\mathrm{mg} / \mathrm{L} \\
\text { as } \mathrm{Na}) \\
(00930)\end{array}$ & $\begin{array}{l}\text { Potas- } \\
\text { slum, } \\
\text { dis- } \\
\text { solvad } \\
\text { (mg/l } \\
\text { as K) } \\
(00935)\end{array}$ & $\begin{array}{c}\text { Chlorida, } \\
\text { dis- } \\
\text { solved } \\
\text { (mg/L } \\
\text { as Cl) } \\
(00940)\end{array}$ & $\begin{array}{c}\text { Sulfate, } \\
\text { dis- } \\
\text { solvad } \\
\text { (mg/l } \\
\text { as } \mathrm{SO}_{4} \text { ) } \\
(00945)\end{array}$ & $\begin{array}{l}\text { Fluoride, } \\
\text { dis- } \\
\text { solved } \\
\text { (mgh } \\
\text { as F) } \\
\text { (00950) }\end{array}$ & $\begin{array}{c}\text { Sillca, } \\
\text { dis- } \\
\text { solved } \\
\left(\mathrm{mgll}^{2} \text { as }\right. \\
\left.\mathrm{SiO}_{2}\right) \\
(00955)\end{array}$ & $\begin{array}{c}\text { Bicar- } \\
\text { bonate } \\
\text { FET- } \\
\text { FLD } \\
\left(\mathrm{mgll}^{2} \text { as }\right. \\
\left.\mathrm{HCO}_{3}\right) \\
(00440)\end{array}$ & $\begin{array}{c}\text { Carbo- } \\
\text { nate } \\
\text { FET- } \\
\text { FLD } \\
(\mathrm{mg} / \mathrm{L} \text { as } \\
\left.\mathrm{CO}_{3}\right) \\
(00445)\end{array}$ & $\begin{array}{c}\text { Nitro- } \\
\text { gen, } \\
\text { nitrate } \\
\text { total } \\
\text { (mg/L } \\
\text { as N) } \\
(00620)\end{array}$ & $\begin{array}{l}\text { Solids, } \\
\text { sum of } \\
\text { consti- } \\
\text { tuents, } \\
\text { dis- } \\
\text { solved } \\
\text { (mg/L) } \\
\text { (70301) }\end{array}$ & $\begin{array}{l}\text { Iron, } \\
\text { total } \\
\text { recover- } \\
\text { eble } \\
(\mu g / L \\
\text { as Fe) } \\
(01045)\end{array}$ & $\begin{array}{c}\text { Manga- } \\
\text { nese, } \\
\text { total } \\
\text { racover- } \\
\text { able } \\
(\mu g / L \\
\text { as Mn) } \\
(01055)\end{array}$ & $\begin{array}{c}\text { Boron, } \\
\text { dis- } \\
\text { solved } \\
(\mu \mathrm{g} / \mathrm{L} \\
\text { as B) } \\
(01020)\end{array}$ \\
\hline
\end{tabular}

\begin{tabular}{|c|c|c|c|c|c|c|c|c|c|c|c|c|}
\hline 1,400 & 37 & 500 & 5,000 & -- & -- & 600 & -- & -- & 8,240 & -- & -- & -- \\
\hline 56 & 8.5 & 25 & 250 & -- & - & 140 & -- & -- & 534 & -- & -- & - \\
\hline 66 & 8.2 & 160 & 700 & -- & -. & 110 & - & -. & 1,310 & -. & -. & -- \\
\hline 300 & 24 & 220 & 2,400 & -- & -- & 310 & - & -- & 3,850 & -- & - & -- \\
\hline
\end{tabular}

$\begin{array}{rrrrrrrrrrrrrrrr}65 & 12 & 120 & 1,600 & -- & -- & 260 & 20 & -- & 3,140 & -- & -- & -- \\ 200 & 32 & 65 & 680 & -- & -- & 440 & 34 & -- & 1,460 & -- & -- & --\end{array}$

\begin{tabular}{|c|c|c|c|c|c|c|c|c|c|c|c|c|}
\hline 390 & 13 & 400 & 11 & 1.6 & 8.1 & 560 & 0 & 0.00 & 1,130 & -- & -- & 4,700 \\
\hline 230 & - & 150 & 460 & - & 8.2 & 460 & 0 & -. & -- & 10 & 190 & -- \\
\hline 300 & -- & 130 & 630 & - & 2.9 & 250 & 0 & -. & -- & 10 & 40 & -- \\
\hline 270 & $=$ & 150 & 360 & - & 1.7 & 360 & 0 & - & -- & $<10$ & 4 & -- \\
\hline 410 & 16 & 220 & 310 & -- & -. & 290 & -- & -- & 1,130 & -- & -. & -- \\
\hline 390 & -- & 550 & 12 & - & 3.4 & 400 & 0 & - & -- & $<10$ & 5 & -- \\
\hline 360 & -- & 430 & 130 & -- & 3.2 & 520 & 0 & - & -. & $<10$ & $<2$ & -- \\
\hline 310 & - & 470 & 12 & $\cdots$ & $<.10$ & 440 & 93 & - & -- & $<10$ & $<2$ & -- \\
\hline 240 & -- & 300 & 36 & - & 2.7 & 480 & 0 & - & -- & $<10$ & $<2$ & -- \\
\hline 380 & 14 & 370 & 11 & -- & -- & 520 & -- & -- & 1,050 & -- & - & -- \\
\hline 410 & 16 & 410 & 76 & -. & - & 490 & -- & - & 1,180 & -- & -- & -- \\
\hline 340 & -. & 230 & 340 & -- & 3.0 & 420 & 0 & - & - & $<10$ & 3 & -- \\
\hline 480 & 15 & 180 & 400 & -. & -- & 300 & -- & - & 1,250 & -- & -- & -- \\
\hline 500 & 19 & 540 & 11 & -- & -- & 360 & - & - & 1,280 & -- & -- & -- \\
\hline 240 & 12 & 160 & 370 & -- & -- & 390 & -- & $\cdots$ & 1,060 & -- & $\cdots$ & -- \\
\hline 220 & - & 120 & 530 & -- & 4.1 & 290 & - & -- & -- & 220 & -- & -- \\
\hline 510 & -- & 340 & 260 & -- & 4.4 & 510 & -- & -- & -- & -- & -- & -- \\
\hline 510 & -- & 340 & 240 & -- & 4.4 & 520 & -- & -- & -- & -- & -- & -- \\
\hline 550 & -- & 440 & 150 & 2.9 & 15 & 600 & 0 & -- & - & -- & -- & -. \\
\hline 350 & 32 & 20 & 1,800 & 1.3 & - & - & -- & -- & 2,780 & -- & -- & -- \\
\hline 520 & 14 & 200 & 660 & .60 & -- & -- & -- & -- & 1,590 & -- & -- & -- \\
\hline-- & -- & 200 & 660 & -- & -- & -- & -- & -. & -- & 100 & 200 & .. \\
\hline-- & -- & -- & -- & $\cdots$ & -- & -- & -- & -- & -- & - & -- & -- \\
\hline-- & -- & 170 & 970 & .50 & -- & -- & -- & 2.00 & -- & 1,500 & 1,000 & -- \\
\hline 430 & 24 & 200 & 960 & .70 & -- & 280 & -- & -- & 1,920 & 700 & -. & -- \\
\hline
\end{tabular}


Table 3. Chemical analyses of ground water in Charles Mix and Douglas Counties--Continued

\begin{tabular}{|c|c|c|c|c|c|c|c|c|c|c|}
\hline $\begin{array}{l}\text { Principal aquifer/ } \\
\text { local identifier }\end{array}$ & $\begin{array}{l}\text { Date of } \\
\text { sample }\end{array}$ & $\begin{array}{c}\text { Depth } \\
\text { of } \\
\text { well } \\
\text { (feet) } \\
(00003)\end{array}$ & $\begin{array}{c}\text { Agency } \\
\text { collecting } \\
\text { sample } \\
\text { (code } \\
\text { number) } \\
\text { (00027) }\end{array}$ & $\begin{array}{c}\text { Agency } \\
\text { analyzing } \\
\text { sample } \\
\text { (code } \\
\text { number) } \\
\text { (00028) }\end{array}$ & $\begin{array}{l}\text { Water } \\
\text { temper- } \\
\text { ature } \\
\left({ }^{\circ} \mathrm{C}\right) \\
(00010)\end{array}$ & $\begin{array}{c}\text { Specific } \\
\text { conduct- } \\
\text { ance } \\
(\mu \mathrm{S} / \mathrm{cm}) \\
(00095)\end{array}$ & $\begin{array}{c}\text { pH } \\
\text { (standard } \\
\text { units) } \\
(00400)\end{array}$ & $\begin{array}{c}\text { Hardness } \\
\text { total } \\
(\mathrm{mg} / \mathrm{L} \text { as } \\
\left.\mathrm{CaCO}_{3}\right) \\
(00900)\end{array}$ & $\begin{array}{c}\text { Calcium, } \\
\text { dissolved } \\
\text { (mg/L } \\
\text { as Ca) } \\
(00915)\end{array}$ & $\begin{array}{l}\text { Magne- } \\
\text { sium, } \\
\text { dissolved } \\
\text { (mg/L } \\
\text { as Mg) } \\
(00925)\end{array}$ \\
\hline
\end{tabular}

Codell aquifer (Cont.)

\begin{tabular}{|c|c|c|c|c|c|c|c|c|c|c|}
\hline 96N64W17AAAD & $10--54$ & 350 & 1028 & 1028 & -. & -- & -- & -. & -- & -- \\
\hline --do-- & $10-\quad-57$ & 350 & 1028 & 1028 & -- & -- & -- & $\cdots$ & -- & $\cdots$ \\
\hline --do-- & $12-17-57$ & 350 & 46004 & 46004 & -- & -- & 7.5 & 480 & 130 & 35 \\
\hline --do-- & $02-26-68$ & 350 & 1028 & 1028 & -- & -- & 7.1 & 470 & 140 & 32 \\
\hline --do-- & $04-16-75$ & 350 & 1028 & 1028 & - & -- & 7.5 & 470 & 130 & 34 \\
\hline --do-- & $05-11-77$ & 350 & 1028 & 1028 & -- & -- & 7.5 & 450 & 120 & 35 \\
\hline --do-- & $12-06-78$ & 350 & 1028 & 1028 & -- & 2,490 & 7.6 & 460 & 130 & 35 \\
\hline --do-- & $03-26-80$ & 350 & 1028 & 1028 & -- & -- & - & - & -- & -- \\
\hline --do-- & $03-16-81$ & 350 & 1028 & 1028 & -. & 2,810 & 7.5 & 470 & 130 & 37 \\
\hline 96N64W26CCBB & $12-19-60$ & 400 & 46008 & 46008 & -. & 2,450 & -- & 60 & 14 & 6.0 \\
\hline 96N64W29ABBB & $-\quad-65$ & 380 & 46008 & 46008 & -. & -- & 8.3 & 87 & 25 & 6.0 \\
\hline 96N64W31CDCB & $06-12-62$ & 691 & 1028 & 9746 & 16.0 & 2,700 & 7.9 & 84 & 22 & 7.1 \\
\hline$--\mathrm{do}--$ & $02-12-63$ & 691 & 1028 & 9746 & 18.0 & 2,720 & 8.0 & 83 & 22 & 6.8 \\
\hline --do-- & $04-12-65$ & 691 & 1028 & 9746 & 19.0 & 2,740 & 8.0 & 80 & 22 & 6.1 \\
\hline --do-- & $12-28-65$ & 691 & 1028 & 9746 & 18.0 & 2,720 & 7.9 & 79 & 20 & 7.1 \\
\hline --do-. & $10-17-66$ & 691 & 1028 & 9746 & 19.0 & 2,700 & 7.9 & 93 & 24 & 8.0 \\
\hline 96N65W 4CCCB & $07-08-38$ & 365 & 1028 & 1028 & $-\cdot$ & $-\cdot$ & $\cdots$ & 61 & 16 & 5.0 \\
\hline --do-- & $10-\quad-53$ & 365 & 1028 & 1028 & - & -- & 7.6 & 61 & 19 & 3.4 \\
\hline $96 \mathrm{~N} 65 \mathrm{~W}$ 4CDCB & $05-\quad-58$ & 365 & 46004 & 46004 & -- & -- & 8.0 & 270 & 74 & 20 \\
\hline --do-- & $12-19-60$ & 365 & 46008 & 46008 & -- & 950 & $\cdots$ & 320 & 100 & 16 \\
\hline $96 \mathrm{~N} 65 \mathrm{~W} 4 \mathrm{CDCD}$ & $10-\quad-53$ & 380 & 1028 & 1028 & $\cdots$ & $\cdots$ & 7.6 & 79 & 24 & 4.7 \\
\hline --do-- & $02-13-57$ & 380 & 46004 & 46004 &.- & -- & 8.3 & 41 & 8.0 & 5.0 \\
\hline --do-- & $12-19-60$ & 380 & 46008 & 46008 & -- & 1,890 & -- & 980 & 300 & 56 \\
\hline --do-- & $01-11-71$ & 380 & 1028 & 1028 & -. & - & 8.4 & 47 & 12 & 3.9 \\
\hline 96N65W 5ADDD & $-\quad-65$ & 350 & 46008 & 46008 & -- & -- & 8.1 & 51 & 14 & 4.0 \\
\hline 96N65W 7AADD & $07-\quad-65$ & 340 & 46008 & 46008 & - & $\cdots$ & 8.4 & 59 & 17 & 4.0 \\
\hline 96N65W10BDDB & $-\quad-65$ & 375 & 46008 & 46008 & - & -- & 8.3 & 76 & 19 & 7.0 \\
\hline 96N65W13BABD & $-\quad-65$ & 480 & 46008 & 46008 & -- & -- & 8.3 & 61 & 18 & 4.0 \\
\hline $96 \mathrm{~N} 65 \mathrm{~W} 16 \mathrm{BCAA}$ & $07-\quad-65$ & 390 & 46008 & 46008 & -. & $\cdots$ & 8.4 & 52 & 16 & 3.0 \\
\hline 96N65W23CADD & $-\quad-65$ & 666 & 46008 & 46008 & -- & -- & 8.3 & 75 & 17 & 8.0 \\
\hline 96N65W23DCBB & $07-\quad-65$ & 666 & 1028 & 1028 & - & $-\cdot$ & 8.3 & 75 & 17 & 8.0 \\
\hline 96N65W29BACC & $-\quad-65$ & 340 & 46008 & 46008 & -. & $\cdots$ & 8.3 & 71 & 20 & 5.0 \\
\hline 96N65W36DCCD & $09-\quad-58$ & 728 & 1028 & 46004 & -- & -- & 7.8 & 190 & 56 & 13 \\
\hline --do-- & $06-12-62$ & 728 & 1028 & 83113 & 16.0 & 2,420 & 7.7 & 170 & 44 & 15 \\
\hline --do-- & $02-12-63$ & 728 & 1028 & 83113 & 18.0 & 2,470 & 8.0 & 170 & 46 & 14 \\
\hline --do-- & $10-17-66$ & 728 & 9746 & 83113 & 18.0 & 2,450 & 7.8 & 170 & 46 & 14 \\
\hline
\end{tabular}




\begin{tabular}{|c|c|c|c|c|c|c|c|c|c|c|c|c|}
\hline $\begin{array}{c}\text { Sodium, } \\
\text { dis- } \\
\text { solved } \\
\text { (mg/L } \\
\text { as } \mathrm{Na}) \\
(00930)\end{array}$ & $\begin{array}{l}\text { Potas- } \\
\text { sium, } \\
\text { dis- } \\
\text { solved } \\
\text { (mg/L } \\
\text { as K) } \\
(00935)\end{array}$ & $\begin{array}{c}\text { Chloride, } \\
\text { dis- } \\
\text { solved } \\
\text { (mg/L } \\
\text { as Cl) } \\
(00940)\end{array}$ & $\begin{array}{l}\text { Sulfate, } \\
\text { dis- } \\
\text { solved } \\
\text { (mg/L } \\
\text { as } \mathrm{SO}_{4} \text { ) } \\
(00945)\end{array}$ & $\begin{array}{l}\text { Fluoride, } \\
\text { dis- } \\
\text { solved } \\
\text { (mg/L } \\
\text { as F) } \\
\text { (00950) }\end{array}$ & $\begin{array}{c}\text { Silica, } \\
\text { dis- } \\
\text { solved } \\
(\mathrm{mg} / \mathrm{L} \text { as } \\
\left.\mathrm{SiO}_{2}\right) \\
(00955)\end{array}$ & $\begin{array}{c}\text { Bicar- } \\
\text { bonate } \\
\text { FET- } \\
\text { FLD } \\
\left(\mathrm{mgll}^{2} \text { as }\right. \\
\left.\mathrm{HCO}_{3}\right) \\
(00440)\end{array}$ & $\begin{array}{c}\text { Carbo- } \\
\text { nate } \\
\text { FET- } \\
\text { FLD } \\
\left(\mathrm{mg}^{\prime} \mathrm{L} \text { as }\right. \\
\left.\mathrm{CO}_{3}\right) \\
(00445)\end{array}$ & $\begin{array}{c}\text { Nitro- } \\
\text { gen, } \\
\text { nitrate } \\
\text { total } \\
\text { (mg/L } \\
\text { as N) } \\
(00620)\end{array}$ & $\begin{array}{l}\text { Solids, } \\
\text { sum of } \\
\text { consti- } \\
\text { tuents, } \\
\text { dis- } \\
\text { solved } \\
\text { (mg/L) } \\
\text { (70301) }\end{array}$ & $\begin{array}{c}\text { Iron, } \\
\text { total } \\
\text { recover- } \\
\text { able } \\
(\mu g / L \\
\text { as Fe) } \\
(01045)\end{array}$ & $\begin{array}{c}\text { Manga- } \\
\text { nese, } \\
\text { total } \\
\text { recover- } \\
\text { able } \\
\text { ( } \mu g / \mathrm{L} \\
\text { as Mn) } \\
(01055)\end{array}$ & $\begin{array}{c}\text { Boron, } \\
\text { dis- } \\
\text { solved } \\
(\mu g / \mathrm{L} \\
\text { as B) } \\
(01020)\end{array}$ \\
\hline
\end{tabular}

\begin{tabular}{|c|c|c|c|c|c|c|c|c|c|c|c|}
\hline 430 & 23 & 170 & -- & -- & -- & -- & -- & -- & -- & -- & -- \\
\hline-- & 23 & 170 & -. & -- & -- & - & -- & -- & -- & -- & -- \\
\hline 450 & 28 & 170 & 980 & 0.50 & -- & 280 & - & 2.50 & 1,930 & 1,800 & 1,500 \\
\hline 430 & 23 & 150 & 990 & .50 & -- & 270 & -- & .00 & 1,890 & 400 & 1,300 \\
\hline 450 & 24 & 160 & 1,000 & .40 & -- & 280 & -- & 1.00 & 1,940 & 600 & 1,700 \\
\hline 450 & 26 & 160 & 970 & .40 & - & 280 & -- & .100 & 1,900 & 400 & 1,900 \\
\hline 440 & 26 & 160 & 980 & .40 & -- & 280 & -- & .700 & 1,910 & 300 & 1,800 \\
\hline-- & -- & -- & -- & $\cdots$ & -- & - & -- & $\cdots$ & -- & -- & -- \\
\hline 450 & 26 & 170 & 920 & .40 & -- & 290 & -- & .100 & 1,870 & 100 & 1,900 \\
\hline 520 & 8.0 & 220 & 510 & 1.2 & -- & - & -- & -- & 1,480 & -- & -- \\
\hline -- & - & 370 & 610 & -- & -- & - & - & -- & 1,890 & -- & - \\
\hline -- & -- & 190 & 770 & 1.1 & 9.4 & 340 & 0 & -- & 1,740 & -- & -- \\
\hline-- & -- & 180 & 790 & 1.2 & 9.2 & 340 & 0 & - & 1,760 & -- & -- \\
\hline-- & - & 190 & 720 & 1.2 & 9.2 & 340 & 0 & - & 1,680 & -- & -- \\
\hline-- & -- & 180 & 790 & 1.1 & 9.6 & 340 & 0 & -- & 1,770 & -- & -- \\
\hline-- & -- & 210 & 780 & 1.3 & 10 & 320 & 0 & -. & 1,780 & -- & -- \\
\hline-- & -- & 1,100 & -- & .40 & -- & -- & - & -- & -- & 400 & -- \\
\hline 700 & 37 & 1,100 & 9.2 & .10 & - & 210 & -- & .00 & 1,950 & 100 & -- \\
\hline 390 & 22 & 310 & 480 & .20 & -- & 270 & -- & -- & 1,430 & -- & -- \\
\hline 34 & 27 & 15 & 260 & 1.1 & -- & -- & - & -- & 555 & -- & -- \\
\hline-- & -- & 1,000 & 17 & .10 & -- & 270 & -- & .00 & -- & 400 & -- \\
\hline 760 & 13 & 910 & 2.0 & .60 & -- & 470 & -- & -- & 1,930 & 2,200 & ND \\
\hline 56 & 49 & 30 & 820 & 1.3 & -- & -- & -- & -- & 1,440 & -- & -- \\
\hline 710 & 13 & 970 & ND & .63 & -- & 340 & -- & .00 & -- & 6,400 & 30 \\
\hline$\cdots$ & - & 1,200 & .0 & -- & -- & -- & -- & -- & 2,760 & -- & -- \\
\hline-- & - & 1,200 & ND & -- & -- & -- & -- & - & -- & 600 & -- \\
\hline-- & - & 1,000 & 19 & - & - & -- & -- & .. & 2,470 & -- & -- \\
\hline-- & -- & 840 & 740 & -- & -- & -- & -- & -- & 1,960 & -- & -- \\
\hline-- & - & 930 & ND & -- & -- & -- & -- & -- & -- & ND & - \\
\hline$\cdots$ & -- & 640 & 190 & -- & -- & -- & -- & + & 2,080 & -- & - \\
\hline-- & - & 640 & 190 & - & -- & -- & -- & -- & -- & 1,300 & -- \\
\hline-- & -- & 960 & .0 & - & -- & -- & -- & - & 2,440 & -- & -- \\
\hline 500 & 24 & 120 & 770 & 1.0 & -- & 370 & -- & - & 1,660 & -- & -- \\
\hline-- & -- & 130 & 740 & .80 & 11 & 350 & 0 & -- & 1,610 & -- & -- \\
\hline-- & -- & 120 & 750 & .80 & 9.9 & 340 & 0 & $\cdots$ & 1,620 & -- & -- \\
\hline-- & -- & 130 & 770 & .80 & 10 & 370 & 0 & -- & 1,660 & $\cdots$ & -- \\
\hline
\end{tabular}


Table 3. Chemical analyses of ground water in Charles Mix and Douglas Counties--Continued

\begin{tabular}{|c|c|c|c|c|c|c|c|c|c|c|}
\hline $\begin{array}{l}\text { Principal aquifer/ } \\
\text { local identifier }\end{array}$ & $\begin{array}{l}\text { Date of } \\
\text { sample }\end{array}$ & $\begin{array}{c}\text { Depth } \\
\text { of } \\
\text { well } \\
\text { (feet) } \\
\text { (00003) }\end{array}$ & $\begin{array}{l}\text { Agency } \\
\text { collecting } \\
\text { sample } \\
\text { (code } \\
\text { number) } \\
\text { (00027) }\end{array}$ & $\begin{array}{c}\text { Agency } \\
\text { analyzlng } \\
\text { sample } \\
\text { (code } \\
\text { number) } \\
\text { (00028) }\end{array}$ & $\begin{array}{c}\text { Water } \\
\text { temper- } \\
\text { ature } \\
\left({ }^{\circ} \mathrm{C}\right) \\
(00010)\end{array}$ & $\begin{array}{c}\text { Specific } \\
\text { conduct- } \\
\text { ance } \\
(\mu S / \mathrm{cm}) \\
(00095)\end{array}$ & $\begin{array}{c}\text { pH } \\
\text { (standard } \\
\text { units) } \\
(00400)\end{array}$ & $\begin{array}{c}\text { Hardness } \\
\text { total } \\
(\mathrm{mg} / \mathrm{L} \text { as } \\
\left.\mathrm{CaCO}_{3}\right) \\
(00900)\end{array}$ & $\begin{array}{c}\text { Calclum, } \\
\text { dissolved } \\
\text { (mg/h } \\
\text { as Ca) } \\
(00915)\end{array}$ & $\begin{array}{l}\text { Magne- } \\
\text { sium, } \\
\text { dissolved } \\
\text { (mg/l } \\
\text { as } \mathrm{Mg}) \\
(00925)\end{array}$ \\
\hline
\end{tabular}

Codell aquifer (Cont.)

\begin{tabular}{|c|c|c|c|c|c|c|c|c|c|c|}
\hline 96N66W 2BDDD2 & $07-\quad-65$ & 608 & 1028 & 1028 & -- & - & 8.5 & 54 & 15 & 4.0 \\
\hline 96N66W23BCBC & $-\quad-65$ & 573 & 46008 & 46008 & -- & -- & 8.4 & 51 & 14 & 4.0 \\
\hline 96N66W23BCCC & $07-\quad-65$ & 577 & 1028 & 1028 & - & -- & 8.4 & 51 & 14 & 4.0 \\
\hline 97N62W 7AAAA & $09-10-68$ & 320 & 1028 & 46002 & - & 2,240 & 8.8 & 29 & 7.0 & 2.9 \\
\hline 97N62W14DDCC & $09-10-68$ & 509 & 1028 & 46002 & -- & 2,500 & 8.2 & 63 & 19 & 3.8 \\
\hline 97N64W 9DAAA & $09-01-60$ & 400 & 1028 & 46004 & -- & -- & -- & 57 & 21 & 1.0 \\
\hline 97N64W17CCDD2 & $07-\quad-65$ & 325 & 46008 & 46008 & -- & -- & 8.0 & 160 & 49 & 9.0 \\
\hline 97N64W32DCAD & $07-\quad-65$ & 325 & 1028 & 1028 & -- & -- & 7.6 & 60 & 11 & 8.0 \\
\hline 97N65W17AADC & $-\quad-65$ & 439 & 46008 & 46008 & - & -- & 8.2 & 59 & 17 & 4.0 \\
\hline 97N65W23DCCD & $-\quad-65$ & 374 & 46008 & 46008 & -- & -- & 8.3 & 71 & 20 & 5.0 \\
\hline 97N65W30CDBD & $-\quad-65$ & 409 & 46008 & 46008 & -- & -- & 8.4 & 51 & 14 & 4.0 \\
\hline 97N65W34CBBB & $-\quad-65$ & 410 & 46008 & 46008 & -- & -- & 8.4 & 120 & 33 & 8.0 \\
\hline 97N66W14AADD & $07-\quad-65$ & 477 & 46008 & 46008 & -- & -- & 8.4 & 82 & 23 & 6.0 \\
\hline 97N67W 6BAD & $01-\quad-55$ & 475 & 1028 & 1028 & -- & -- & 7.4 & 570 & 160 & 43 \\
\hline --do-- & $12-11-57$ & 475 & 46004 & 46004 & -- & -- & 7.7 & 650 & 190 & 44 \\
\hline --do-- & $02-22-67$ & 475 & 1028 & 1028 & -- & -- & 6.9 & 610 & 170 & 46 \\
\hline --do-- & $06-06-72$ & 475 & 1028 & 1028 & -- & -- & 7.5 & 540 & 160 & 32 \\
\hline 97N67W 6BAD2 & $12-11-54$ & 472 & 1028 & 1028 & - & - & 7.5 & 580 & 160 & 44 \\
\hline --do-- & $12-\quad-57$ & 472 & 46004 & 46004 & -- & -- & 7.5 & 580 & 160 & 44 \\
\hline --do-- & $04-26-66$ & 472 & 46004 & 46004 & -- & - & 7.1 & 520 & 150 & 38 \\
\hline --do-- & $03-05-75$ & 472 & 1028 & 1028 & -- & -- & 7.6 & 580 & 160 & 44 \\
\hline --do-- & $05-12-77$ & 472 & 1028 & 1028 & -- & -- & 7.2 & 530 & 140 & 43 \\
\hline $98 \mathrm{~N} 62 \mathrm{~W} 24 \mathrm{BBCC} 2$ & $09-09-68$ & 412 & 1028 & 46002 & -- & 2,030 & 8.1 & 120 & 32 & 8.6 \\
\hline $98 \mathrm{~N} 62 \mathrm{~W} 34 \mathrm{BABB} 2$ & 10. -60 & 336 & 1028 & 46002 & -- & -- & 8.4 & 56 & 14 & 5.0 \\
\hline $98 \mathrm{~N} 62 \mathrm{~W} 34 \mathrm{BABB} 3$ & $10-\quad-60$ & 341 & 46004 & 46004 & -- & -- & 8.5 & 57 & 23 & .0 \\
\hline 98N62W34BABB3 & $05-\quad-66$ & 341 & 46004 & 46004 & -- & -- & 7.7 & 58 & 15 & 5.1 \\
\hline 98N62W34BBAB & $02-07-58$ & 330 & 1028 & 1028 & -- & -- & 7.9 & 61 & 18 & 4.0 \\
\hline --do-- & $10-31-60$ & 330 & 1028 & 1028 & -- & -- & 8.5 & 60 & 23 & ND \\
\hline --do-- & $05-04-66$ & 330 & 1028 & 1028 & -- & -- & 7.7 & 58 & 15 & 5.1 \\
\hline 98N62W34BBAD & $09-\quad-38$ & 220 & 1028 & 1028 & -- & -- & - & 71 & 17 & 7.0 \\
\hline --do-- & $03-20-40$ & 220 & 1028 & 1028 & -- & -- & -- & 130 & 21 & 18 \\
\hline --do-- & $10-15-54$ & 220 & 1028 & 1028 & -- & -- & 7.8 & 45 & 10 & 4.6 \\
\hline --do-- & $10-31-60$ & 220 & 1028 & 1028 & -- & - & 8.4 & 56 & 14 & 5.0 \\
\hline --do-- & $11-01-71$ & 220 & 1028 & 1028 & -- & - & 8.1 & 73 & 16 & 8.0 \\
\hline 98N62W34BCBD & $10-15-70$ & 335 & 1028 & 1028 & -- & -- & 7.9 & 56 & 16 & 4.0 \\
\hline
\end{tabular}




\begin{tabular}{|c|c|c|c|c|c|c|c|c|c|c|c|c|}
\hline $\begin{array}{c}\text { Sodium, } \\
\text { dis- } \\
\text { solved } \\
\text { (mg/L } \\
\text { as } \mathrm{Na} \text { ) } \\
(00930)\end{array}$ & $\begin{array}{l}\text { Potas- } \\
\text { sium, } \\
\text { dis- } \\
\text { solved } \\
\text { (mg/L } \\
\text { as K) } \\
(00935)\end{array}$ & $\begin{array}{c}\text { Chloride, } \\
\text { dis- } \\
\text { solved } \\
\text { (mg/L } \\
\text { as Cl) } \\
\text { (00940) }\end{array}$ & $\begin{array}{c}\text { Sulfate, } \\
\text { dis- } \\
\text { solved } \\
\text { (mg/L } \\
\left.\text { as } \mathrm{SO}_{4}\right) \\
(00945)\end{array}$ & $\begin{array}{c}\text { Fluoride, } \\
\text { dis- } \\
\text { solved } \\
\text { (mg/L } \\
\text { as F) } \\
(00950)\end{array}$ & $\begin{array}{c}\text { Silica, } \\
\text { dis- } \\
\text { solved } \\
\left(\mathrm{mg}^{2} \mathrm{~L} \text { as }\right. \\
\left.\mathrm{SiO}_{2}\right) \\
(00955)\end{array}$ & $\begin{array}{c}\text { Bicar- } \\
\text { bonate } \\
\text { FET- } \\
\text { FLD } \\
\text { (mg/l as } \\
\left.\mathrm{HCO}_{3}\right) \\
(00440)\end{array}$ & $\begin{array}{c}\text { Carbo- } \\
\text { nste } \\
\text { FET- } \\
\text { FLD } \\
\text { (mg/L as } \\
\left.\mathrm{CO}_{3}\right) \\
(00445)\end{array}$ & $\begin{array}{c}\text { Nitro- } \\
\text { gen, } \\
\text { nitrste } \\
\text { tots। } \\
\text { (mg/L } \\
\text { as N) } \\
(00620)\end{array}$ & $\begin{array}{l}\text { Solids, } \\
\text { sum of } \\
\text { consti- } \\
\text { tuents, } \\
\text { dis- } \\
\text { solved } \\
\text { (mg/l) } \\
\text { (70301) }\end{array}$ & $\begin{array}{c}\text { Iron, } \\
\text { total } \\
\text { recover- } \\
\text { able } \\
(\mu \mathrm{g} / \mathrm{L} \\
\text { es Fe) } \\
(01045)\end{array}$ & $\begin{array}{c}\text { Mangs- } \\
\text { nese, } \\
\text { total } \\
\text { recover- } \\
\text { able } \\
(\mu g / L \\
\text { as Mn) } \\
(01055)\end{array}$ & $\begin{array}{c}\text { Boron, } \\
\text { dis- } \\
\text { solved } \\
(\mu \mathrm{g} / \mathrm{L} \\
\text { as B) } \\
(01020)\end{array}$ \\
\hline
\end{tabular}

\begin{tabular}{|c|c|c|c|c|c|c|c|c|c|c|c|c|}
\hline -- & - & 860 & 19 & -- & -- & -- & -- & -- & -- & 120 & -- & -. \\
\hline -. & -- & 780 & .0 & -. & -- & - & -- & $\ldots$ & 2,440 & -- & - & -- \\
\hline- & -- & 790 & ND & -- & -- & -- & -- & - & -- & 200 & -- & -- \\
\hline 470 & 12 & 220 & 300 & $=$ & -- & 500 & -- & -- & 1,260 & -- & -- & -- \\
\hline 520 & 17 & 370 & 310 & -- & -- & 500 & -- & - & 1,490 & -- & -- & -- \\
\hline 530 & -- & 460 & 190 & -- & -- & -- & -- & -- & 1,630 & -- & -- & -- \\
\hline-- & -- & 290 & 300 & -- & -- & - & -- & -- & -- & -- & -- & -- \\
\hline-- & $\cdots$ & 1,100 & ND & $\cdots$ & -- & .. & -- & -- & -- & 280 & ND & -. \\
\hline-- & -- & 660 & 110 & -- & -- & - & -- & -- & 2,200 & -- & -- & -- \\
\hline-- & -- & 790 & .0 & -- & -- & -. & - & $\cdots$ & 2,340 & -- & $\cdots$ & -- \\
\hline-- & -- & 640 & 210 & -- & -- & -- & -- & -- & 2,140 & -- & -- & -- \\
\hline- & -- & 1,100 & 19 & - & -- & $m$ & - & -- & 2,820 & -- & -- & -. \\
\hline -- & -- & 440 & 700 & -- & -- & -- & -- & -- & -- & 800 & ND & -. \\
\hline-- & -- & 220 & - & 1.5 & -- & 230 & -- & 0.00 & 0 & 760 & + & -- \\
\hline 400 & 25 & 260 & 940 & 1.5 & -- & 230 & -- & .00 & 1,970 & 2,900 & ND & -- \\
\hline 360 & 23 & 190 & 940 & 1.4 & -- & 180 & -- & .00 & 1,820 & 2,200 & ND & - \\
\hline 380 & 21 & 200 & 940 & 1.1 & -- & 220 & -- & 1.00 & 1,840 & 1,200 & ND & -- \\
\hline 390 & 24 & 220 & 930 & 1.4 & - & 220 & -- & .00 & 1,870 & 3,100 & ND & -- \\
\hline 390 & 24 & 220 & 940 & 1.4 & -- & 220 & - & -- & 1,880 & -. & -- & -- \\
\hline 400 & 21 & 200 & 960 & 1.5 & -- & 200 & -- & .150 & 1,870 & 2,800 & 30 & -- \\
\hline 380 & 22 & 210 & 960 & 1.2 & -- & 220 & -- & 1.00 & 1,890 & 900 & ND & -- \\
\hline 400 & 23 & 200 & 950 & 1.0 & -- & 150 & -- & .100 & 1,830 & 2,800 & 50 & - \\
\hline 410 & 17 & 96 & 690 & -- & -- & 230 & - & -- & 1,370 & -- & - & -- \\
\hline 520 & 1.5 & 160 & 530 & 1.7 & -- & 490 & -- & -- & 1,470 & -- & - & -- \\
\hline 530 & 1.5 & 130 & 620 & 1.7 & -- & 450 & -- & -- & 1,530 & -- & -- & -- \\
\hline 520 & 14 & 120 & 620 & 1.8 & -- & 450 & -- & -- & 1,520 & -- & - & -- \\
\hline 530 & 14 & 130 & 620 & 1.9 & -- & 470 & -- & 1.50 & 1,540 & ND & ND & -- \\
\hline 530 & 1.5 & 130 & 620 & 1.7 & -- & 450 & - & 2.00 & -- & ND & ND & -- \\
\hline 510 & 14 & 120 & 620 & 1.8 & -- & 450 & -. & 2.50 & 1,510 & 40 & 10 & -- \\
\hline-- & -- & 140 & 600 & 2.0 & 58 & -- & -- & -- & -- & 200 & ND & -- \\
\hline-- & -- & 140 & 570 & 1.2 & -- & -- & -. & -- & -- & 100 & ND & -- \\
\hline-- & -- & 130 & 600 & 1.9 & -- & 470 & -- & .600 & -- & 300 & -- & -- \\
\hline 530 & 1.3 & 170 & 530 & 1.7 & -- & 490 & -- & 2.00 & 1,480 & 1,100 & ND & -- \\
\hline 500 & 11 & 130 & 600 & 1.8 & -- & 480 & -- & $<1.00$ & 1,500 & 100 & ND & -- \\
\hline 480 & 12 & 170 & 510 & 1.6 & -- & 490 & - & ND & 1,440 & 700 & ND & -- \\
\hline
\end{tabular}


Table 3. Chemical analyses of ground water in Charles Mix and Douglas Counties--Continued

\begin{tabular}{|c|c|c|c|c|c|c|c|c|c|c|}
\hline $\begin{array}{l}\text { Principal aquifer/ } \\
\text { local Identifier }\end{array}$ & $\begin{array}{l}\text { Date of } \\
\text { aample }\end{array}$ & $\begin{array}{c}\text { Depth } \\
\text { of } \\
\text { well } \\
\text { (feet) } \\
\text { (00003) }\end{array}$ & $\begin{array}{c}\text { Agency } \\
\text { collecting } \\
\text { sample } \\
\text { (code } \\
\text { number) } \\
\text { (00027) }\end{array}$ & $\begin{array}{l}\text { Agency } \\
\text { analyzing } \\
\text { sample } \\
\text { (code } \\
\text { number) } \\
\text { (00028) }\end{array}$ & $\begin{array}{c}\text { Water } \\
\text { temper- } \\
\text { ature } \\
\left({ }^{\circ} \mathrm{C}\right) \\
(00010)\end{array}$ & $\begin{array}{c}\text { Specific } \\
\text { conduct- } \\
\text { ance } \\
(\mu \mathrm{S} / \mathrm{cm}) \\
(00095)\end{array}$ & $\begin{array}{c}\text { pH } \\
\text { (standard } \\
\text { units) } \\
(00400)\end{array}$ & $\begin{array}{c}\text { Hardness } \\
\text { total } \\
(\mathrm{mg} / \mathrm{L} \text { as } \\
\mathrm{CaCO} \text { ) } \\
(00900)\end{array}$ & $\begin{array}{l}\text { Calcium, } \\
\text { diasolved } \\
\text { (mg/L } \\
\text { as Ca) } \\
\text { (00915) }\end{array}$ & $\begin{array}{c}\text { Magne- } \\
\text { sium, } \\
\text { dissolvad } \\
(\mathbf{m g} / \mathbf{L} \\
\text { aa } \mathbf{M g}) \\
(00925)\end{array}$ \\
\hline
\end{tabular}

Codell aquifer (Cont.)

\begin{tabular}{|c|c|c|c|c|c|c|c|c|c|c|}
\hline 98N62W34BCBD & $09-11-72$ & 335 & 1028 & 1028 & -- & -. & 7.9 & 56 & 16 & 4.0 \\
\hline --do-- & $01-13-76$ & 335 & 1028 & 1028 & -- & -- & 8.2 & 59 & 15 & 5.4 \\
\hline --do-- & $10-03-77$ & 335 & 1028 & 1028 & -- & 2,250 & 8.0 & 58 & 15 & 5.2 \\
\hline --do-- & $12-13-77$ & 335 & 1028 & 1028 & -- & -- & -- & -- & -- & -- \\
\hline --do-- & $11-27-79$ & 335 & 1028 & 1028 & -- & 2,150 & 7.8 & 56 & 14 & 5.0 \\
\hline --do-- & $11-03-80$ & 335 & 1028 & 1028 & -- & -- & -- & -- & -- & -- \\
\hline 98N63W26AAAA & $09-30-59$ & 314 & 1028 & 46003 & -- & 2,250 & -- & 320 & 92 & 22 \\
\hline --do-- & $12-19-60$ & 314 & 46008 & 46003 & -- & 2,250 & -- & 300 & 84 & 23 \\
\hline 98N63W29AABB & $09-10-68$ & 319 & 1028 & 46002 & 12.0 & 2,070 & 8.2 & 140 & 50 & 2.9 \\
\hline 98N64W12DADC2 & $09-\quad-57$ & 415 & 46004 & 46004 & -- & -- & 7.7 & 210 & 58 & 17 \\
\hline 98N64W12DADC3 & $09-\quad-37$ & 415 & 1028 & 1028 & -- & -- & -- & 250 & 63 & 23 \\
\hline --do-- & $01-05-40$ & 415 & 1028 & 1028 & -- & -- & -- & 330 & 94 & 23 \\
\hline --do-- & $10-\quad-53$ & 415 & 1028 & 1028 & -- & -- & 7.8 & 250 & 68 & 20 \\
\hline --do-- & $09-\quad-57$ & 415 & 46004 & 46004 & -- & -- & 8.0 & 230 & 62 & 19 \\
\hline --do-- & $09-17-57$ & 415 & 1028 & 1028 & -- & -- & 7.5 & 220 & 58 & 19 \\
\hline --do-- & $10-19-60$ & 415 & 1028 & 1028 & -- & -- & 7.7 & 220 & 61 & 17 \\
\hline --do-. & $02-10-75$ & 415 & 1028 & 1028 & -- & -- & 7.9 & 220 & 61 & 18 \\
\hline 98N64W 12DADC4 & $09-\quad-57$ & 415 & 46004 & 46004 & -- & -- & 7.5 & 220 & 58 & 19 \\
\hline 98N64W12DADC5 & $10-\quad-60$ & 415 & 46004 & 46004 & -- & -- & 7.7 & 220 & 61 & 17 \\
\hline 98N65W 1CDDA2 & $09-09-68$ & 420 & 1028 & 46002 & 14.0 & 2,500 & 8.3 & 65 & 18 & 4.8 \\
\hline 98N65W33BCAA3 & 09-11-69 & 535 & 1028 & 1028 & 16.0 & 2,080 & 8.4 & 210 & 56 & 16 \\
\hline 98N66W19AAAD & $10-11-68$ & 425 & 1028 & 46002 & -- & 2,240 & 7.3 & 840 & 270 & 40 \\
\hline 98N67W 7AAAA & $10-04-68$ & 538 & 1028 & 46002 & 12.0 & 2,990 & 7.5 & 460 & 140 & 27 \\
\hline 98N67W32AAAD & $10-10-68$ & 462 & 1028 & 46002 & -- & 2,450 & 7.8 & 35 & 11 & 1.9 \\
\hline 99N62W 2DDDA & 09-09-68 & 293 & 1028 & 46002 & -- & 2,230 & 8.0 & 170 & 53 & 8.6 \\
\hline 99N62W10ADAA & $12-19-60$ & 460 & 1028 & 46008 & -- & 2,820 & -- & 750 & 240 & 36 \\
\hline 99N62W21AABD & $12-19-60$ & 410 & 1028 & 46003 & -- & 1,900 & -- & 230 & 50 & 26 \\
\hline 99N63W20DDCA & $09-10-68$ & 347 & 1028 & 46002 & -- & 1,180 & 7.8 & 390 & 110 & 27 \\
\hline 99N63W25DDDD & $07-31-68$ & 340 & 1028 & 46002 & 11.0 & 2,240 & 7.2 & 440 & 140 & 22 \\
\hline 99N63W28DDDD2 & $05-15-80$ & 318 & 1028 & 1028 & -- & 2,200 & 7.7 & 400 & 80 & 48 \\
\hline 99N64W 3 & $03-17-81$ & 383 & 46004 & 46004 & -- & 2,080 & 7.9 & 160 & -- & -- \\
\hline 99N64W 3BADD & $09-\quad-57$ & 382 & 1028 & 46004 & -- & -. & 8.0 & 140 & 38 & 11 \\
\hline --do-- & $04-24-67$ & 382 & 1028 & 1028 & -- & 1,980 & 7.9 & 160 & 46 & 11 \\
\hline 99N64W 3BADD2 & $09-08-69$ & 395 & 1028 & 1028 & -. & -- & 7.7 & 140 & 38 & 10 \\
\hline 99N64W 3BBA & $09-\quad-57$ & 377 & 1028 & 46004 & -- & -- & 7.8 & 140 & 38 & 11 \\
\hline
\end{tabular}




\begin{tabular}{|c|c|c|c|c|c|c|c|c|c|c|c|c|}
\hline $\begin{array}{c}\text { Sodium, } \\
\text { dis- } \\
\text { solved } \\
\text { ( } \mathrm{mg} / \mathrm{L} \\
\text { as } \mathrm{Na}) \\
(00930)\end{array}$ & $\begin{array}{l}\text { Potas- } \\
\text { sium, } \\
\text { dis- } \\
\text { solved } \\
\text { (mg/L } \\
\text { as K) } \\
(00935)\end{array}$ & $\begin{array}{c}\text { Chloride, } \\
\text { dis- } \\
\text { solved } \\
\text { (mg/L } \\
\text { as CI) } \\
\text { (00940) }\end{array}$ & $\begin{array}{c}\text { Sulfate, } \\
\text { dis- } \\
\text { solved } \\
\text { (mg/L } \\
\text { as } \mathrm{SO}_{4} \text { ) } \\
(00945)\end{array}$ & $\begin{array}{c}\text { Fluoride, } \\
\text { dis- } \\
\text { solved } \\
\text { (mg/L } \\
\text { as F) } \\
\text { (00950) }\end{array}$ & $\begin{array}{c}\text { Silica, } \\
\text { dis- } \\
\text { solved } \\
\left(\mathrm{mg}^{2} \mathrm{~L} \text { as }\right. \\
\left.\mathrm{SiO}_{2}\right) \\
(\mathrm{OO955})\end{array}$ & $\begin{array}{c}\text { Bicar- } \\
\text { bonate } \\
\text { FET- } \\
\text { FLD } \\
\left(\mathrm{mg}^{2} \text { as }\right. \\
\left.\mathrm{HCO}_{3}\right) \\
(00440)\end{array}$ & $\begin{array}{c}\text { Carbo- } \\
\text { nate } \\
\text { FET- } \\
\text { FLD } \\
(\mathrm{mg} / \mathrm{L} \text { as } \\
\left.\mathrm{CO}_{3}\right) \\
(00445)\end{array}$ & $\begin{array}{c}\text { Nitro- } \\
\text { gen, } \\
\text { nitrate } \\
\text { total } \\
\text { (mg/L } \\
\text { as N) } \\
(00620)\end{array}$ & $\begin{array}{l}\text { Solids, } \\
\text { sum of } \\
\text { consti- } \\
\text { tuents, } \\
\text { dis- } \\
\text { solved } \\
\text { (mg/L) } \\
\text { (70301) }\end{array}$ & $\begin{array}{l}\text { Iron, } \\
\text { total } \\
\text { recover- } \\
\text { able } \\
(\mu g / L \\
\text { as Fe) } \\
(01045)\end{array}$ & $\begin{array}{c}\text { Manga- } \\
\text { nese, } \\
\text { total } \\
\text { recover- } \\
\text { abie } \\
(\mu g / L \\
\text { as } M n) \\
(01055)\end{array}$ & $\begin{array}{c}\text { Boron, } \\
\text { dis- } \\
\text { solved } \\
(\mu \mathrm{g} / \mathrm{L} \\
\text { as B) } \\
(01020)\end{array}$ \\
\hline
\end{tabular}

\begin{tabular}{|c|c|c|c|c|c|c|c|c|c|c|c|c|}
\hline 510 & 9.4 & 180 & 510 & 1.7 & -. & 500 & -. & $<1.00$ & 1,480 & ND & ND & -- \\
\hline 520 & 13 & 180 & 520 & 1.2 & - & 500 & -- & $<1.00$ & 1,500 & 60 & $<20$ & -- \\
\hline 520 & 13 & 180 & 490 & 1.6 & -- & 500 & -- & .700 & 1,480 & 100 & $<20$ & - \\
\hline -- & -- & -- & -- & 1.6 & -- & -- & -- & $<.100$ & - & -- & - & -- \\
\hline 510 & 13 & 180 & 480 & 1.7 & -- & 500 & - & .500 & 1,450 & $<20$ & $<20$ & -- \\
\hline-- & -- & -- & -- & 1.6 & -- & -- & -- & .300 & -- & - & -- & -- \\
\hline 300 & 3.0 & 110 & 780 & -- & -- & 200 & -- & -- & 1,400 & -- & -- & -- \\
\hline 350 & 16 & 100 & 770 & 1.2 & -- & . & -- & -. & 1,430 & - & -- & -- \\
\hline 400 & 19 & 60 & 670 & -- & -- & 290 & - & -- & 1,350 & -- & -. & -- \\
\hline 520 & 18 & 240 & 700 & 1.2 & -- & 350 & -- & -- & 1,730 & - & -- & - \\
\hline -- & -- & 260 & 700 & 1.2 & -- & -- & -- & .. & -- & -- & -- & -- \\
\hline$\cdots$ & - & 260 & 670 & 1.2 & $\cdots$ & -- & -- & -- & -- & 100 & ND & -. \\
\hline -- & -- & 260 & 690 & 1.3 & -- & 330 & -. & 3.00 & -. & 1,100 & -- & -- \\
\hline 520 & 18 & 260 & 700 & 1.2 & -- & 390 & $-\cdot$ &.- & 1,770 & -- & -- & -- \\
\hline 520 & 18 & 250 & 700 & 1.2 & -- & 350 & - & 1.50 & 1,740 & 300 & ND & -- \\
\hline 540 & 19 & 260 & 710 & 1.3 & -- & 350 & -- & ND & 1,780 & 600 & ND & -. \\
\hline 510 & 170 & 250 & 700 & .90 & -- & 350 & -- & $<1.00$ & 1,880 & 600 & 20 & -- \\
\hline 520 & 18 & 250 & 700 & 1.2 & - & 350 & -- & -- & 1,740 & -- & - & -- \\
\hline 540 & 19 & 260 & 710 & 1.3 & - & 350 & -- & -- & 1,780 & -- & -. & -- \\
\hline 520 & 14 & 370 & 240 & -- & $\cdots$ & 360 & - & - & 1,340 & -- & $=$ & -. \\
\hline 320 & 9.1 & 440 & 55 & -. & - & 380 & -- & - & 1,080 & -- & -- & -- \\
\hline 200 & 22 & 200 & 970 & -- & -- & 100 & -- & $\cdots$ & 1,750 & -- & -- & -- \\
\hline 480 & 26 & 130 & 1,300 & - & -- & 110 & -- & - & 2,160 & -- & -- & -- \\
\hline 570 & 9.5 & 440 & 2.2 & -- & -- & 510 & -- & $\cdots$ & 1,290 & -- & -- & -- \\
\hline 520 & 16 & 120 & 710 & -- & -- & 410 & -- & -- & 1,630 & -- & -- & -- \\
\hline 340 & 24 & 13 & 1,200 & 1.1 & -. & -- & -- & -. & 2,000 & - & -- & -- \\
\hline 310 & 20 & 25 & 620 & 1.0 & - & -- & -- & - & 1,180 & -. & -. & -- \\
\hline 130 & 18 & 16 & 450 & -- & -- & 280 & -- & -- & 887 & -- & - & -- \\
\hline 300 & 25 & 110 & 730 & -- & -- & 270 & -- & -- & 1,460 & - & -- & -- \\
\hline 400 & 35 & 210 & 860 & -- & - & 120 & -- & 3.85 & 1,700 & 510 & -- & 2,900 \\
\hline 400 & 16 & 68 & 550 & -. & -- & 420 & -- & -. & - & -. & -- & -- \\
\hline 400 & 18 & 60 & 570 & 2.1 & -- & 440 & -- & -- & 1,310 & -. & -. & -- \\
\hline 400 & 16 & 65 & 580 & 2.1 & 9.6 & 410 & 0 & -- & 1,340 & $\cdots$ & -- & 4,100 \\
\hline 380 & 16 & 58 & 570 & 1.9 & -- & 420 & -- & ND & 1,280 & 200 & ND & .. \\
\hline 420 & 19 & 60 & 570 & 2.2 & - & 450 & -- & -- & 1,340 & -- & -- & -- \\
\hline
\end{tabular}


Table 3. Chemical analyses of ground water in Charles Mix and Douglas Counties--Continued

\begin{tabular}{|c|c|c|c|c|c|c|c|c|c|c|}
\hline $\begin{array}{l}\text { Principal equlfer/ } \\
\text { local identifier }\end{array}$ & $\begin{array}{l}\text { Date of } \\
\text { sample }\end{array}$ & $\begin{array}{c}\text { Depth } \\
\text { of } \\
\text { well } \\
\text { (feet) } \\
(00003)\end{array}$ & $\begin{array}{c}\text { Agency } \\
\text { collecting } \\
\text { sample } \\
\text { (code } \\
\text { number) } \\
\text { (00027) }\end{array}$ & $\begin{array}{c}\text { Agency } \\
\text { analyzing } \\
\text { sample } \\
\text { (code } \\
\text { number) } \\
\text { (00028) }\end{array}$ & $\begin{array}{c}\text { Water } \\
\text { temper- } \\
\text { ature } \\
\left({ }^{\circ} \mathrm{C}\right) \\
(00010)\end{array}$ & $\begin{array}{c}\text { Specific } \\
\text { conduct- } \\
\text { ance } \\
(\mu \mathrm{S} / \mathrm{cm}) \\
(00095)\end{array}$ & $\begin{array}{c}\text { pH } \\
\text { (standerd } \\
\text { units) } \\
(00400)\end{array}$ & $\begin{array}{c}\text { Hardness } \\
\text { total } \\
\text { (mg/L es } \\
\left.\mathrm{CaCO}_{3}\right) \\
(00900)\end{array}$ & $\begin{array}{l}\text { Calcium, } \\
\text { dissolved } \\
\text { (mg/L } \\
\text { as Ca) } \\
(00915)\end{array}$ & $\begin{array}{c}\text { Magne- } \\
\text { sium, } \\
\text { dissolved } \\
\text { (mg/L } \\
\text { as Mg) } \\
(00925)\end{array}$ \\
\hline
\end{tabular}

Codell aquifer (Cont.)

\begin{tabular}{|c|c|c|c|c|c|c|c|c|c|c|}
\hline 99N64W 3BBA & $04-15-66$ & 377 & 46004 & 46004 & 16.5 & 1,940 & 8.1 & 130 & 35 & 11 \\
\hline 99N64W 3BCAA & $10--53$ & 382 & 1028 & 1028 & -- & -- & 7.7 & 160 & 46 & 10 \\
\hline --do-- & $09-10-57$ & 382 & 1028 & 1028 & -. & -- & 7.8 & 140 & 38 & 11 \\
\hline --do-- & $09-08-69$ & 382 & 1028 & 1028 & -- & -- & 7.3 & 150 & 43 & 10 \\
\hline --do-- & $01-12-76$ & 382 & 1028 & 1028 & -- & -- & 7.9 & 160 & 44 & 13 \\
\hline - do-m & $08-31-81$ & 382 & 1028 & 1028 & -- & -. & -. & -- & -- & -- \\
\hline 99N64W 6ABBB & $09-09-68$ & 400 & 1028 & 46002 & 13.0 & 2,500 & 8.0 & 120 & 38 & 6.7 \\
\hline 99N64W25BCCC & $09-10-68$ & 462 & 1028 & 46002 & 10.0 & 2,750 & 7.8 & 240 & 78 & 12 \\
\hline $99 \mathrm{~N} 65 \mathrm{~W} 3 \mathrm{ABBB}$ & $09-10-68$ & 410 & 1028 & 46002 & -- & 2,860 & 7.8 & 91 & 30 & 3.8 \\
\hline 99N65W13DDCC2 & $09-10-68$ & 415 & 1028 & 46002 & -- & 2,550 & 8.0 & 79 & 22 & 5.8 \\
\hline 99N66W 4DDAD & $09-10-68$ & 350 & 1028 & 46002 & 13.0 & 1,780 & 8.4 & 56 & 16 & \\
\hline 99N66W25CCCC & $09-10-68$ & 474 & 1028 & 46002 & -. & 2,560 & 8.5 & 65 & 18 & 1.0 \\
\hline 99N68W13CCCA & $10-30-37$ & 455 & 1028 & 1028 & -- & -- & -- & 77 & 21 & ( \\
\hline --do-- & $10-\quad-54$ & 455 & 46004 & 46004 & -- & -- & 7.7 & 88 & 27 & 5.0 \\
\hline --do-- & $04-\quad-66$ & 455 & 46004 & 46004 & - & -- & 8.2 & 64 & 14 & 7.6 \\
\hline --do-- & $01-11-71$ & 455 & 1028 & 1028 & -- & -- & 7.9 & 82 & 23 & 6.0 \\
\hline --do-- & $01-13-76$ & 455 & 1028 & 1028 & -- & -- & 8.1 & 82 & 21 & 7.4 \\
\hline --do-- & $10-03-77$ & 455 & 1028 & 1028 & -- & 3,130 & 8.0 & 79 & 20 & 7.0 \\
\hline 100N62W24AABD & $09-09-68$ & 227 & 1028 & 46002 & -- & 2,110 & 7.9 & 100 & 32 & 5.8 \\
\hline $100 \mathrm{~N} 62 \mathrm{~W} 33 \mathrm{AAAA}$ & $09-09-68$ & 345 & 1028 & 46002 & 12.0 & 2,040 & 8.2 & 69 & 18 & 5.8 \\
\hline 100N63W15ABBB & $09-09-68$ & 396 & 1028 & 46002 & 14.0 & 1,780 & 8.4 & 77 & 11 & 12 \\
\hline 100N63W32BBBB & $09-10-68$ & 472 & 1028 & 46002 & -. & 1,680 & 8.1 & 55 & 14 & 4.8 \\
\hline 100N64W13BAAD & $09-09-68$ & 400 & 1028 & 46002 & -- & 1,460 & 7.9 & 67 & 19 & 4.8 \\
\hline 100N64W27ADDD & $09-09-68$ & 385 & 1028 & 46002 & 12.0 & 1,640 & 8.5 & 57 & 18 & 2.9 \\
\hline 100N65W 8DAAD & $09-10-68$ & 365 & 1028 & 46002 & -- & 3,230 & 8.0 & 120 & 35 & 7.7 \\
\hline $100 \mathrm{~N} 65 \mathrm{~W} 24 \mathrm{CCCB}$ & $09-10-68$ & 364 & 1028 & 46002 & 13.0 & 2,960 & 7.9 & 160 & 43 & 12 \\
\hline
\end{tabular}

Dakota aquifer

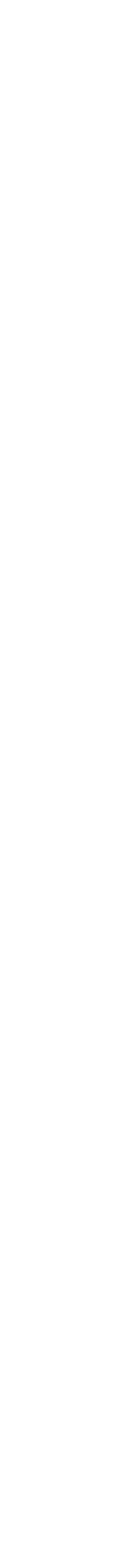

$\begin{array}{lrrrrrrrrrr}\text { 93N62W 7BBDD } & 09-20-66 & 660 & 1028 & 46002 & 18.0 & 1,750 & 7.6 & 820 & 280 & 30 \\ \text { 93N62W 7DAC } & 09-15-60 & 603 & 1028 & 1028 & 18.5 & 1,660 & 7.1 & 740 & 220 & 45 \\ \quad-- \text { do-- } & 04-05-67 & 603 & 1028 & 1028 & -- & 1,660 & 8.0 & 730 & 220 & 44 \\ \text { 94N61W 7CAA } & 02-17-67 & 693 & 1028 & 1028 & 18.5 & 1,920 & 7.4 & 990 & 300 & 58 \\ \text { 94N62W30DDDA } & 11-06-79 & 640 & 46003 & 84610 & 11.0 & 2,580 & 5.9 & 1,600 & 260 & 230 \\ & & & & & & & & & & \\ \text { 94N64W26DBA } & 09-14-60 & 680 & 1028 & 1028 & 12.0 & 1,660 & 7.3 & 700 & 210 & 43 \\ \text {--do-- } & 04-05-67 & 680 & 1028 & 1028 & 19.0 & 1,600 & 8.0 & 710 & 210 & 44 \\ \text {--do-- } & 06-06-74 & 680 & 1028 & 80020 & 19.5 & 1,650 & 7.4 & 710 & 210 & 43 \\ \text { 95N65W 4DAAA } & 01-02-47 & 854 & 46002 & 46002 & -- & -- & - & 920 & 280 & 53 \\ \text { 96N62W 7AADD } & 12-19-60 & 805 & 1028 & 46008 & -- & 2,200 & - & 1,000 & 300 & 67\end{array}$

124 Records of Wells and Chemicel Analyses of Ground Water in Charles Mix and Douglas Counties, South Dekota 


\begin{tabular}{|c|c|c|c|c|c|c|c|c|c|c|c|c|}
\hline $\begin{array}{c}\text { Sodium, } \\
\text { dis- } \\
\text { solved } \\
\text { (mg/L } \\
\text { as Na) } \\
(00930)\end{array}$ & $\begin{array}{l}\text { Potas- } \\
\text { sium, } \\
\text { dis- } \\
\text { solved } \\
\text { (mg/L } \\
\text { as K) } \\
(00935)\end{array}$ & $\begin{array}{c}\text { Chloride, } \\
\text { dis- } \\
\text { solved } \\
\text { (mg/L } \\
\text { as Cl) } \\
\text { (00940) }\end{array}$ & $\begin{array}{l}\text { Sulfate, } \\
\text { dis- } \\
\text { solved } \\
(\mathrm{mg} / \mathrm{L} \\
\left.\text { as SO }_{4}\right) \\
(00945)\end{array}$ & $\begin{array}{c}\text { Fluoride, } \\
\text { dis- } \\
\text { solved } \\
\text { (mg/L } \\
\text { as F) } \\
\text { (00950) }\end{array}$ & $\begin{array}{c}\text { Silica, } \\
\text { dis- } \\
\text { solved } \\
(\mathrm{mg} / \mathrm{L} \text { ss } \\
\left.\mathrm{SiO}_{2}\right) \\
(00955)\end{array}$ & $\begin{array}{c}\text { Bicar- } \\
\text { bonate } \\
\text { FET- } \\
\text { FLD } \\
\left(\mathrm{mg}^{\prime} / \mathrm{L} \text { as }\right. \\
\left.\mathrm{HCO}_{3}\right) \\
(00440)\end{array}$ & $\begin{array}{c}\text { Carbo- } \\
\text { nate } \\
\text { FET- } \\
\text { FLD } \\
\left(\mathrm{mg}^{2} \mathrm{~L} \text { as }\right. \\
\left.\mathrm{CO}_{3}\right) \\
(00445)\end{array}$ & $\begin{array}{c}\text { Nitro- } \\
\text { gen, } \\
\text { nitrate } \\
\text { total } \\
\text { (mg/L } \\
\text { as N) }\end{array}$ & $\begin{array}{l}\text { sun } \\
\text { con } \\
\text { tue } \\
\text { di } \\
\text { sol } \\
\text { (me }\end{array}$ & $\begin{array}{l}\text { Iron, } \\
\text { total } \\
\text { recover- } \\
\text { able } \\
(\mu \mathrm{g} / \mathrm{L} \\
\text { as } \mathrm{Fe}) \\
(01045)\end{array}$ & $\begin{array}{c}\text { Manga- } \\
\text { nese, } \\
\text { total } \\
\text { recover- } \\
\text { able } \\
(\mu g / L \\
\text { as Mn) } \\
(01055)\end{array}$ & $\begin{array}{r}\text { Bo } \\
\text { d } \\
\text { sol } \\
(\mu \\
\text { as } \\
(01\end{array}$ \\
\hline
\end{tabular}

\begin{tabular}{|c|c|c|c|c|c|c|c|c|c|c|c|c|}
\hline 380 & 15 & 66 & 540 & 2.2 & 6.1 & 410 & 0 & -- & 1,260 & -- & -. & 400 \\
\hline -- & -. & 62 & 540 & 2.3 & -- & 430 & -- & ND & -. & 100 & -. & -- \\
\hline 420 & 19 & 60 & 570 & 2.2 & -- & 450 & -- & ND & 1,340 & 600 & ND & -- \\
\hline 360 & 19 & 45 & 530 & 1.8 & -- & 420 & -- & ND & 1,220 & 400 & ND & -- \\
\hline 380 & 18 & 57 & 550 & 1.3 & -- & 430 & -- & $<1.00$ & 1,270 & 300 & 20 & -- \\
\hline -- & -. & -. & -- & 1.9 & -- & -- & -- & $<.100$ & -- & -- & -- & -- \\
\hline 540 & 18 & 390 & 440 & -- & -- & 160 & -- & -- & 1,510 & -- & -. & -- \\
\hline 500 & 22 & 310 & 640 & -. & -- & 330 & -- & -- & 1,720 & -- & -- & -- \\
\hline 560 & 18 & 500 & 330 & -- & -- & 380 & -- & -- & 1,630 & -- & -- & -- \\
\hline 570 & 14 & 360 & 370 & -- & -- & 410 & -- & -- & 1,540 & -- & -- & -- \\
\hline 370 & 12 & 190 & 300 & -- & -. & 280 & -- & -- & 1,030 & -- & -- & -- \\
\hline 540 & 14 & 360 & 410 & -- & -- & 490 & -- & -- & 1,590 & -. & -- & -- \\
\hline -- & -- & 610 & 260 & 3.2 & 9.0 & -- & -- & -- & -- & 1,000 & ND & -- \\
\hline -- & -- & 590 & 250 & 2.8 & -- & 530 & -- & -- & 1,910 & -- & -. & -- \\
\hline 640 & 17 & 560 & 250 & 2.6 & -- & 510 & -- & -- & 1,740 & -. & -- & -- \\
\hline 640 & 12 & 580 & 260 & 2.8 & -- & -- & -- & 1.00 & 1,780 & 500 & ND & -. \\
\hline 660 & 14 & 590 & 230 & 1.8 & -- & 530 & -- & 1.00 & 1,790 & 200 & $<20$ & -- \\
\hline 670 & 14 & 600 & 240 & 3.0 & -- & 530 & -- & .100 & 1,810 & 200 & 30 & -- \\
\hline 430 & 12 & 100 & 620 & $\cdots$ & $\cdots$ & 400 & -- & -- & 1,390 & -- & -- & -- \\
\hline 450 & 11 & 130 & 430 & -- & -- & 470 & -. & -- & 1,270 & -- & -- & -- \\
\hline 390 & 10 & 50 & 530 & -- & -- & 290 & -. & - & 1,150 & -- & -- & -- \\
\hline 340 & 12 & 120 & 330 & -- & -- & 400 & -- & -- & 1,020 & -- & -- & -. \\
\hline 320 & 12 & 18 & 440 & -- & -- & 320 & -- & -- & 972 & -- & -- & -- \\
\hline 350 & 14 & 30 & 480 & -- & -- & 320 & -- & -- & 1,050 & -- & $\cdots$ & -- \\
\hline 630 & 26 & 590 & 440 & -- & - & 390 & -- & -- & 1,920 & -- & -- & - \\
\hline 640 & 24 & 520 & 380 & -- & -- & 360 & -- & -- & 1,800 & -- & -- & -- \\
\hline
\end{tabular}

$\begin{array}{rrrrrrrrrrrrr}56 & 27 & 66 & 730 & - & -- & - & - & -- & 1,300 & -- & - & - \\ 100 & 22 & 62 & 700 & 4.2 & 9.3 & 200 & 0 & -- & 1,260 & -- & -- & 720 \\ 100 & 22 & 58 & 680 & 3.0 & 9.3 & 190 & 0 & -- & 1,240 & -- & -- & 860 \\ 93 & 20 & 92 & 810 & -- & -- & 170 & 0 & -- & 1,450 & 8,600 & -- & - \\ 120 & -- & 14 & 2,100 & -- & 11 & 480 & 0 & -- & -- & 30 & 8 & - \\ & & & & & & & & & & & & 730 \\ 97 & 23 & 49 & 700 & 5.0 & 9.9 & 180 & 0 & -- & 1,230 & -- & -- & 700 \\ 100 & 22 & 47 & 680 & 3.8 & 9.4 & 180 & 0 & -- & 1,210 & -- & -- & 710 \\ 90 & 22 & 51 & 660 & 2.9 & 9.6 & 190 & 0 & -- & 1,190 & -- & -- & -- \\ 46 & -- & 46 & 820 & 3.1 & 12 & 150 & -- & -- & 1,360 & - & - & 2,000 \\ 110 & 19 & 110 & 970 & 1.5 & -- & -- & -- & -- & 1,650 & -- & -- & -\end{array}$


Table 3. Chemical analyses of ground water in Charles Mix and Douglas Counties--Continued

\begin{tabular}{|c|c|c|c|c|c|c|c|c|c|c|}
\hline $\begin{array}{l}\text { Principal aquifer/ } \\
\text { local identifier }\end{array}$ & $\begin{array}{l}\text { Date of } \\
\text { semple }\end{array}$ & $\begin{array}{c}\text { Depth } \\
\text { of } \\
\text { weli } \\
\text { (feet) } \\
(00003)\end{array}$ & $\begin{array}{c}\text { Agency } \\
\text { collecting } \\
\text { sample } \\
\text { (code } \\
\text { number) } \\
\text { (00027) }\end{array}$ & $\begin{array}{c}\text { Agency } \\
\text { analyzing } \\
\text { sample } \\
\text { (code } \\
\text { number) } \\
\text { (00028) }\end{array}$ & $\begin{array}{c}\text { Water } \\
\text { temper- } \\
\text { ature } \\
\left({ }^{\circ} \mathrm{C}\right) \\
(00010)\end{array}$ & $\begin{array}{c}\text { Specific } \\
\text { conduct- } \\
\text { ance } \\
(\mu \mathrm{S} / \mathrm{cm}) \\
(00095)\end{array}$ & $\begin{array}{c}\text { pH } \\
\text { (standard } \\
\text { units) } \\
(00400)\end{array}$ & $\begin{array}{c}\text { Hardness } \\
\text { total } \\
\text { (mg/L as } \\
\left.\mathrm{CaCO}_{3}\right) \\
(00900)\end{array}$ & $\begin{array}{l}\text { Calcium, } \\
\text { dissolved } \\
\text { (mg/L } \\
\text { as Ca) } \\
(00915)\end{array}$ & $\begin{array}{l}\text { Magne- } \\
\text { slum, } \\
\text { dissolved } \\
\text { (mg/L } \\
\text { as } \mathrm{Mg}) \\
(00925)\end{array}$ \\
\hline
\end{tabular}

Dakota aquifer (Cont.)

\begin{tabular}{|c|c|c|c|c|c|c|c|c|c|c|}
\hline $96 \mathrm{~N} 62 \mathrm{~W} 9 \mathrm{BABB}$ & $05-09-60$ & 560 & 1028 & 1028 & -- & 1,960 & 7.2 & 840 & 240 & 58 \\
\hline 96N63W 8CDA & $09-14-60$ & 672 & 1028 & 1028 & 16.0 & 2,000 & 7.2 & 810 & 240 & 51 \\
\hline --do-- & $04-05-67$ & 672 & 1028 & 1028 & 16.0 & 2,050 & 8.0 & 830 & 240 & 55 \\
\hline --do-- & $06-06-74$ & 672 & 1028 & 84913 & 15.5 & 2,040 & 7.5 & 800 & 230 & 52 \\
\hline --do-- & $08-04-79$ & 672 & 1028 & 80020 & 15.0 & 2,080 & 7.4 & 780 & 230 & 48 \\
\hline --do-- & $05-08-80$ & 672 & 1028 & 80020 & 15.0 & 1,990 & 7.4 & -- & -- & -- \\
\hline 96N63W33CCBA2 & $05-09-60$ & 870 & 1028 & 1028 & 20.0 & 2,370 & 6.8 & 1,200 & 350 & 75 \\
\hline 96N64W 6ACDA & $08-28-69$ & 732 & 1028 & 46002 & 23.0 & 1,910 & 8.4 & 880 & 270 & 51 \\
\hline 96N64W 7ABCD2 & $06-12-46$ & 950 & 1028 & 1028 & 21.0 & 2,190 & -- & 1,000 & 320 & 57 \\
\hline $96 \mathrm{~N} 65 \mathrm{~W} 10 \mathrm{~B}$ & $06-12-46$ & 909 & 1028 & 1028 & 14.0 & -- & 8.0 & 61 & 21 & 2.0 \\
\hline 96N66W 8CBB & $03-30-61$ & 845 & 1028 & 46001 & -- & 1,800 & 7.3 & 990 & 300 & 59 \\
\hline 96N66W29DAB & $06-02-67$ & 1,130 & 1028 & 46002 & -. & 1,740 & 7.4 & 840 & 270 & 41 \\
\hline --do-- & $06-19-67$ & 1,130 & 1028 & 46002 & -. & 1,840 & 7.3 & 830 & 260 & 43 \\
\hline $97 \mathrm{~N} 64 \mathrm{~W} 27 \mathrm{CCCB}$ & $12-19-60$ & 860 & 46008 & 46008 & 17.0 & 2,200 & -- & 840 & 250 & 52 \\
\hline 97N66W 7BAAA & $03-19-68$ & 822 & 1028 & 46001 & -. & 1,920 & 7.1 & 880 & -- & -- \\
\hline $97 \mathrm{~N} 67 \mathrm{~W} 5 \mathrm{BCC}$ & $05-01-62$ & 1,100 & 1028 & 46001 & -- & 1,600 & -- & 670 & 180 & 53 \\
\hline $98 \mathrm{~N} 62 \mathrm{~W} 5 \mathrm{BCBB}$ & $09-13-60$ & 833 & 1028 & 1028 & 16.0 & 2,550 & 7.2 & 1,400 & 430 & 84 \\
\hline --do-- & $04-16-65$ & 833 & 1028 & 1028 & 16.0 & 2,600 & 7.4 & 1,400 & 430 & 83 \\
\hline --do-- & $04-25-67$ & 833 & 1028 & 1028 & 14.0 & 2,560 & 7.7 & 1,400 & 420 & 89 \\
\hline 98N64W12DADC & $09-\quad-57$ & 757 & 1028 & 1028 & 20.0 & -- & -- & 1,300 & 370 & 100 \\
\hline 98N67W10CABB & $10-04-68$ & 940 & 1028 & 1028 & 13.0 & 2,170 & 7.4 & 1,000 & 310 & 59 \\
\hline 98N68W13ABAA & $06-12-69$ & 890 & 1028 & 46002 & 19.0 & 1,880 & 7.8 & 800 & 190 & 78 \\
\hline 98N69W14DCAA & $09-11-69$ & 1,280 & 1028 & 46002 & 19.0 & 1,870 & 7.9 & 980 & 300 & 56 \\
\hline 99N63W14ADA & $09-10-68$ & 640 & 1028 & 46002 & 15.0 & 2,500 & 7.8 & 1,200 & 390 & 51 \\
\hline 99N68W13CBBA & $10-30-37$ & 838 & 1028 & 1028 & -- & -- & -- & 1,600 & 380 & 160 \\
\hline --do-- & $10-\quad-54$ & 838 & 46004 & 46004 & -. & -- & 7.8 & 1200 & 380 & 69 \\
\hline --do-- & $10-21-54$ & 838 & 1028 & 1028 & -- & -- & 7.8 & 1,200 & 380 & 69 \\
\hline --do-- & $01-11-71$ & 838 & 1028 & 1028 & -- & -- & 7.1 & 1,100 & 350 & 53 \\
\hline --do-- & $08-\quad-71$ & 838 & 1028 & 1028 & -- & -- & 7.0 & 990 & 300 & 56 \\
\hline 99N68W13CCCA2 & $12-28-56$ & 948 & 46004 & 46004 & -- & -- & 7.5 & 1,100 & 330 & 71 \\
\hline - do-- & $04-26-66$ & 948 & 46004 & 46004 & -- & - & 7.8 & 1,200 & 370 & 63 \\
\hline --do-- & $01-11-71$ & 948 & 1028 & 1028 & -- & -- & 7.2 & 1,200 & 370 & 63 \\
\hline --do-- & $01-13-76$ & 948 & 1028 & 1028 & -. & -- & 7.5 & 1,200 & 370 & 73 \\
\hline --do-- & $10-03-77$ & 948 & 1028 & 1028 & -- & 2,390 & 7.4 & 1,200 & 360 & 71 \\
\hline 99N68W31DDDB & $05-09-60$ & 960 & 1028 & 1028 & 14.0 & 2,100 & 7.0 & 1,100 & 310 & 70 \\
\hline
\end{tabular}




\begin{tabular}{|c|c|c|c|c|c|c|c|c|c|c|c|c|}
\hline $\begin{array}{c}\text { Sodium, } \\
\text { dis- } \\
\text { soived } \\
\text { (mg/L } \\
\text { as Na) } \\
(00930)\end{array}$ & $\begin{array}{l}\text { Potas- } \\
\text { sium, } \\
\text { dis- } \\
\text { solved } \\
\text { (mg/L } \\
\text { as K) } \\
(00935)\end{array}$ & $\begin{array}{c}\text { Chloride, } \\
\text { dis- } \\
\text { solved } \\
\text { (mg/L } \\
\text { as Cl) } \\
\text { (00940) }\end{array}$ & $\begin{array}{c}\text { Sulfate, } \\
\text { dis- } \\
\text { soived } \\
(\mathrm{mg} / \mathrm{L} \\
\left.\text { as } \mathrm{SO}_{4}\right) \\
(00945)\end{array}$ & $\begin{array}{c}\text { Fluoride, } \\
\text { dis- } \\
\text { solved } \\
\text { (mg/l } \\
\text { as F) } \\
(00950)\end{array}$ & $\begin{array}{c}\text { Silica, } \\
\text { dis- } \\
\text { solved } \\
\left(\mathrm{mg}^{2} \mathrm{~L} \text { as }\right. \\
\left.\mathrm{SiO}_{2}\right) \\
(00955)\end{array}$ & $\begin{array}{c}\text { Bicar- } \\
\text { bonnate } \\
\text { FET- } \\
\text { FLD } \\
\left(\text { mgll as }^{2} \text { aCO }_{3}\right) \\
(00440)\end{array}$ & $\begin{array}{c}\text { Carbo- } \\
\text { nate } \\
\text { FET- } \\
\text { FLD } \\
\left(\mathrm{mg}^{2} \text { L as }\right. \\
\left.\mathrm{CO}_{3}\right) \\
(00445)\end{array}$ & $\begin{array}{c}\text { Nitro- } \\
\text { gen, } \\
\text { nitrate } \\
\text { total } \\
\text { (mg/L } \\
\text { as N) } \\
(00620)\end{array}$ & $\begin{array}{l}\text { Solids, } \\
\text { sum of } \\
\text { consti- } \\
\text { tuents, } \\
\text { dis- } \\
\text { solved } \\
\text { (mg/L) } \\
(70301)\end{array}$ & $\begin{array}{l}\text { Iron, } \\
\text { totai } \\
\text { recover- } \\
\text { able } \\
\text { ( } \mu \mathrm{g} / \mathrm{L} \\
\text { as } \mathrm{Fe}) \\
(01045)\end{array}$ & $\begin{array}{c}\text { Manga- } \\
\text { nese, } \\
\text { total } \\
\text { recover- } \\
\text { able } \\
(\mu g / L \\
\text { as } \mathrm{Mn}) \\
(01055)\end{array}$ & $\begin{array}{c}\text { Boron, } \\
\text { dis- } \\
\text { soived } \\
(\mu g / L \\
\text { as B) } \\
(01020)\end{array}$ \\
\hline
\end{tabular}

\begin{tabular}{|c|c|c|c|c|c|c|c|c|c|c|c|c|}
\hline 120 & 20 & 93 & 860 & 2.7 & 6.4 & 170 & 0 & -- & 1,480 & -- & -- & 530 \\
\hline 160 & 20 & 100 & 850 & 3.5 & 8.8 & 180 & 0 & .. & 1,530 & .- & .- & 690 \\
\hline 170 & 20 & 94 & 840 & 3.0 & 8.6 & 190 & 0 & -- & 1,530 & -- & -- & 7,200 \\
\hline 150 & 20 & 100 & 780 & 2.6 & 8.8 & 190 & 0 & -- & 1,450 & -- & -- & 740 \\
\hline 150 & 20 & 97 & 820 & 2.9 & 9.0 & 200 & 0 & -- & 1,490 & 2,800 & -. & 730 \\
\hline -- & -- & -- & -- & -- & -- & -- & -. & -- & -- & -- & -- & .- \\
\hline 110 & 19 & 160 & 1,100 & 2.7 & 9.3 & 150 & -- & -- & 1,900 & -- & -- & 120 \\
\hline 87 & 19 & 150 & 790 & -- & -- & 120 & -- & .- & 1,430 & -- & -- & -- \\
\hline 110 & 22 & 140 & 930 & -. & 8.1 & 130 & -- & -- & 1,650 & -- & -- & -- \\
\hline 710 & 13 & 1100 & 13 & -- & 15 & 190 & -- & -- & 1,970 & -- & -- & -- \\
\hline 49 & 13 & 83 & 870 & -- & -- & 160 & 0 & -- & 1,450 & -- & -- & -- \\
\hline 94 & -- & -- & 980 & -- & -- & 180 & -- & -- & -- & -- & -- & -- \\
\hline 74 & -- & -- & 800 & -- & -- & 200 & $\cdots$ & -- & -- & -- & -. & -- \\
\hline 140 & 22 & 120 & 900 & 1.5 & -- & -- & -- & -- & 1,570 & .. & -- & -- \\
\hline 110 & -- & -. & -- & -- & - & -- & - & -- & -- & -- & - & -- \\
\hline 48 & 10 & 120 & 300 & -- & .- & 300 & 20 & -- & 879 & -- & -. & -- \\
\hline 110 & 20 & 150 & 1,300 & 3.4 & 9.9 & 160 & 0 & -- & 2,180 & -- & -- & 230 \\
\hline 110 & 20 & 150 & 1,200 & 2.9 & 10 & 160 & 0 & -. & 2,080 & -- & -. & 240 \\
\hline 120 & 20 & 150 & 1,200 & 2.8 & 10 & 160 & 0 & -- & 2,090 & -- & -- & 2,500 \\
\hline -- & -- & 170 & 1,200 & -- & 9.0 & -- & -. & -- & -- & -- & -- & -- \\
\hline 96 & 18 & 160 & 990 & -- & -- & 78 & -. & -. & 1,670 & -- & -- & -. \\
\hline 47 & 23 & 98 & 750 & -- & -. & 120 & -- & -- & 1,240 & -- & -- & -- \\
\hline 90 & 14 & 140 & 660 & -- & -- & 130 & -- & -- & 1,320 & -- & -- & -- \\
\hline 98 & 20 & 110 & 1,200 & -- & -- & 150 & -- & -- & 1,940 & -- & - & -- \\
\hline -- & -- & 210 & 1,100 & 3.4 & 11 & -- & -- & -- & -- & 3,200 & 200 & -- \\
\hline -- & -. & 250 & 1,100 & 2.6 & -- & 160 & -- & -- & 2,220 & -- & -- & -- \\
\hline 180 & 11 & 250 & 1,100 & 2,6 & -- & 160 & -- & 0.00 & 2,040 & 4,400 & -- & -- \\
\hline 150 & 17 & 220 & 1,000 & 2.3 & -- & 100 & -- & 1.00 & 1,900 & 9,600 & 100 & -- \\
\hline 170 & 7.0 & 240 & 990 & 2.4 & -- & 59 & -- & .100 & 1,790 & 5,600 & 10 & -- \\
\hline 140 & 19 & 210 & 1,000 & 2.8 & -- & 110 & -- & .00 & 1,860 & 10,000 & 100 & -- \\
\hline 130 & 28 & 200 & 1,100 & 2.8 & -- & 150 & -- & .00 & 1,930 & 1,800 & 100 & -- \\
\hline 130 & 17 & 200 & 1,000 & 2.5 & -- & 150 & -- & 1.00 & 1,900 & 2,800 & ND & -- \\
\hline 130 & 19 & 210 & 1,100 & 1.7 & -- & 140 & -- & 1.00 & 1,940 & 2,200 & 160 & -- \\
\hline 130 & 18 & 220 & 1,000 & 2.8 & -- & 140 & - & .100 & 1,910 & 1,900 & 200 & -- \\
\hline 90 & 18 & 120 & 960 & 2.7 & 9.6 & 150 & 0 & -- & 1,650 & -- & - & 150 \\
\hline
\end{tabular}


Table 3. Chemical analyses of ground water in Charles Mix and Douglas Counties--Continued

\begin{tabular}{|c|c|c|c|c|c|c|c|c|c|c|}
\hline $\begin{array}{l}\text { Princlpal aquiferl } \\
\text { local identifier }\end{array}$ & $\begin{array}{l}\text { Date of } \\
\text { sample }\end{array}$ & $\begin{array}{c}\text { Depth } \\
\text { of } \\
\text { well } \\
\text { (feet) } \\
(00003)\end{array}$ & $\begin{array}{l}\text { Agency } \\
\text { collecting } \\
\text { sample } \\
\text { (code } \\
\text { number) } \\
\text { (00027) }\end{array}$ & $\begin{array}{l}\text { Agency } \\
\text { analyzing } \\
\text { semple } \\
\text { (code } \\
\text { number) } \\
\text { (00028) }\end{array}$ & $\begin{array}{c}\text { Weter } \\
\text { temper- } \\
\text { ature } \\
\left({ }^{\circ} \mathrm{C}\right) \\
(00010)\end{array}$ & $\begin{array}{c}\text { Specific } \\
\text { conduct- } \\
\text { ance } \\
(\mu \mathrm{S} / \mathrm{cm}) \\
(00095)\end{array}$ & $\begin{array}{c}\text { pH } \\
\text { (atandard } \\
\text { units) } \\
(00400)\end{array}$ & $\begin{array}{c}\text { Hardness } \\
\text { total } \\
(\mathrm{mgl} / \mathrm{aa} \\
\left.\mathrm{CaCO}_{3}\right) \\
(00900)\end{array}$ & $\begin{array}{c}\text { Calcium, } \\
\text { diasolved } \\
\text { (mg/L } \\
\text { aa Ca) } \\
(00915)\end{array}$ & $\begin{array}{l}\text { Magne- } \\
\text { sium, } \\
\text { dissolved } \\
\text { (mg/l } \\
\text { as Mg) } \\
(00925)\end{array}$ \\
\hline \multicolumn{11}{|l|}{ Dakota aquifer (Cont.) } \\
\hline \multicolumn{11}{|l|}{ (................. } \\
\hline 99N68W31DDDB & $04-05-67$ & 960 & 1028 & 1028 & 13.5 & 2,160 & 7.9 & 1,100 & 320 & 67 \\
\hline 99N69W18BBBD & $09-12-68$ & 920 & 1028 & 46002 & 13.0 & 1,980 & 7.6 & 820 & 270 & 36 \\
\hline 99N69W23DDDB & $05-31-67$ & 860 & 1028 & 46002 & - & 1,890 & 7.9 & 1,000 & 320 & 58 \\
\hline 100N67W14BAAA & $09-11-69$ & 850 & 1028 & 46002 & -- & 2,310 & 8.0 & 1,300 & 390 & 88 \\
\hline $100 N 68 W 25 C B B$ & $09-20-68$ & 840 & 1028 & 46002 & -- & 2,650 & 7.2 & 1,200 & 390 & 48 \\
\hline 100N68W35DDDD & $09-20-68$ & 840 & 1028 & 46002 & 21.0 & 2,320 & 8.0 & 960 & 310 & 46 \\
\hline $100 \mathrm{~N} 70 \mathrm{~W} 25 \mathrm{BBC}$ & $09-11-69$ & 900 & 1028 & 46002 & -- & 2,160 & 8.2 & 1,000 & 310 & 63 \\
\hline \multicolumn{11}{|l|}{ Niobrara aquifer } \\
\hline 93N62W 3CBBB & $11-06-70$ & 358 & 46003 & 84610 & 115 & 1070 & 84 & 210 & 66 & 12 \\
\hline $97 \mathrm{~N} 65 \mathrm{~W} 24 \mathrm{CBCC}$ & $06-\quad-65$ & 247 & 46008 & 46008 & - & -- & 8.0 & 810 & 300 & 15 \\
\hline 97N66W 7B & $-\quad-68$ & 210 & 1028 & 1028 & -- & 3,500 & 7.7 & 96 & 22 & 10 \\
\hline 97N66W 7BAAA2 & $10-14-67$ & 210 & 1028 & 46002 & 16.0 & 3,500 & 7.7 & 94 & 22 & 9.6 \\
\hline $98 \mathrm{~N} 62 \mathrm{~W} 27 \mathrm{ABBB}$ & $09-09-68$ & 180 & 1028 & 46002 & 13.0 & 2,000 & 8.0 & 140 & 40 & 9.6 \\
\hline $98 \mathrm{~N} 63 \mathrm{~W} 6 \mathrm{D}$ & $02-14-73$ & 140 & 1028 & 1028 & -- & 2,220 & 7.4 & 120 & 10 & 22 \\
\hline 98N63W19AAAA & $10-02-69$ & 192 & 1028 & 46002 & 12.0 & 2,130 & 8.3 & 320 & 72 & 34 \\
\hline --do-- & $10-23-79$ & 192 & 1028 & 1028 & 21.0 & 2,150 & - & 330 & 95 & 22 \\
\hline 100N66W10DDCC & $09-10-68$ & 180 & 1028 & 46002 & 13.0 & 2,440 & 7.8 & 75 & 22 & 4.8 \\
\hline $100 \mathrm{~N} 66 \mathrm{~W} 21 \mathrm{BDBB}$ & $09-10-68$ & 280 & 1028 & 46002 & 13.0 & 2,000 & 8.1 & 95 & 27 & 6.7 \\
\hline \multicolumn{11}{|l|}{ Pierre Shale } \\
\hline $100 \mathrm{~N} 71 \mathrm{~W} 13 \mathrm{ADAD}$ & $09-12-68$ & 90 & 1028 & 46002 & 150 & 2.340 & 7.6 & 830 & 250 & 49 \\
\hline
\end{tabular}




\begin{tabular}{|c|c|c|c|c|c|c|c|c|c|c|c|c|}
\hline $\begin{array}{l}\text { Sodium, } \\
\text { dis- } \\
\text { solved } \\
\text { (mg/t } \\
\text { as } \mathrm{Na}) \\
(00930)\end{array}$ & $\begin{array}{l}\text { Potas- } \\
\text { sium, } \\
\text { dis- } \\
\text { solved } \\
\text { (mg/L } \\
\text { as K) } \\
(00935 \text { ) }\end{array}$ & $\begin{array}{c}\text { Chioride, } \\
\text { dis- } \\
\text { solved } \\
\text { (mg/L } \\
\text { as Cl) } \\
(00940)\end{array}$ & $\begin{array}{l}\text { Sulfate, } \\
\text { dis- } \\
\text { solved } \\
\text { (mg/L } \\
\left.\text { as } \mathrm{SO}_{4}\right) \\
(00945)\end{array}$ & $\begin{array}{c}\text { Fluorlde, } \\
\text { dis- } \\
\text { solved } \\
\text { (mg/L } \\
\text { as F) } \\
(00950)\end{array}$ & $\begin{array}{c}\text { Silica, } \\
\text { dis- } \\
\text { solved } \\
(\mathrm{mg} / \mathrm{L} \text { as } \\
\left.\mathrm{SiO}_{2}\right) \\
(00955)\end{array}$ & $\begin{array}{c}\text { Bicar- } \\
\text { bonate } \\
\text { FET- } \\
\text { FLD } \\
\text { (mg/L as } \\
\left.\text { HCO }_{3}\right) \\
(00440)\end{array}$ & $\begin{array}{c}\text { Carbo- } \\
\text { nate } \\
\text { FET- } \\
\text { FLD } \\
\text { (mgll as } \\
\left.\mathrm{CO}_{3}\right) \\
(00445)\end{array}$ & $\begin{array}{l}\text { Nitro- } \\
\text { gen, } \\
\text { nitrate } \\
\text { total } \\
\text { (mg/L } \\
\text { as N) } \\
(00620)\end{array}$ & $\begin{array}{l}\text { Solids, } \\
\text { sum of } \\
\text { consti- } \\
\text { tuents, } \\
\text { dis- } \\
\text { solved } \\
\text { (mg/L) } \\
\text { (70301) }\end{array}$ & $\begin{array}{c}\text { Iron, } \\
\text { total } \\
\text { recover- } \\
\text { able } \\
(\mu \mathrm{g} / \mathrm{L} \\
\text { as Fe) } \\
(01045)\end{array}$ & $\begin{array}{c}\text { Manga- } \\
\text { nese, } \\
\text { totai } \\
\text { recover- } \\
\text { able } \\
\text { ( } \mu g / L \\
\text { es Mn) } \\
(01055)\end{array}$ & $\begin{array}{c}\text { Boron, } \\
\text { dis- } \\
\text { solved } \\
(\mu \mathrm{g} / \mathrm{L} \\
\text { as B) } \\
(01020)\end{array}$ \\
\hline
\end{tabular}

\begin{tabular}{|c|c|c|c|c|c|c|c|c|c|c|c|}
\hline 96 & 20 & 120 & 940 & 2.9 & 9.2 & 140 & 0 & -- & 1,650 & -- & -- \\
\hline 76 & 21 & 110 & 930 & -. & -- & 68 & -- & -- & 1,480 & -- & -- \\
\hline 92 & 21 & 170 & 970 & -. & -- & 170 & $\ldots$ & -- & 1,710 & -- & -- \\
\hline 170 & 20 & 88 & 1,200 & -- & -- & 180 & -- & - & 2,040 & - & -- \\
\hline 130 & 21 & 140 & 110 & - & -- & 120 & -- & -- & 899 & -- & -- \\
\hline 110 & 23 & 96 & 1,100 & -- & -- & 98 & - & -- & 1,730 & -- & -- \\
\hline 87 & 23 & 110 & 960 & -- & -- & 180 & -- & - & 1,640 & -- & -- \\
\hline
\end{tabular}

\begin{tabular}{|c|c|c|c|c|c|c|c|c|c|c|c|c|}
\hline 140 & -- & 36 & 240 & .. & .20 & 54 & 5 & -. & -. & $<10$ & 26 & -- \\
\hline -- & -- & 170 & 1,800 & -- & -- & -- & -- & -- & -- & 500 & 300 & -- \\
\hline 790 & 16 & 830 & $\cdots$ & -. & -. & -. & -- & -- & -- & 19,000 & -- & -- \\
\hline 790 & 16 & 830 & 20 & -- & -. & 450 & -- & -- & 1,910 & -- & -- & -. \\
\hline 410 & 16 & 59 & 600 & -- & -- & 420 & -- & -- & 1,340 & -. & -. & -. \\
\hline 420 & 25 & 38 & 650 & -- & -- & 440 & -- & -- & 1,380 & -- & -- & .. \\
\hline 310 & 22 & 90 & 650 & -. & -. & 310 & -- & -- & 1,330 & -- & -- & -. \\
\hline 360 & -- & 120 & 660 & .67 & .. & -- & -- & $<.500$ & -. & $<50$ & $<50$ & -- \\
\hline 500 & 18 & 380 & 170 & -- & -- & 540 & -- & -. & 1,360 & -. & -. & -- \\
\hline 400 & 16 & 240 & 330 & -. & .. & 380 & -. & .. & 1,200 & -. & - & -- \\
\hline 180 & 26 & 26 & 1,100 & -. & -. & 260 & -- & -- & 1,730 & -- & -- & .- \\
\hline
\end{tabular}

